\title{
The Globular Cluster Luminosity Functions of Brightest Cluster Galaxies
}

by

\author{
John Paul Blakeslee
}

B. A., The University of Chicago (1991)

Submitted to the Department of Physics

in partial fulfillment of the requirements for the degree of

Doctor of Philosophy

at the

\section{MASSACHUSETTS INSTITUTE OF TECHNOLOGY}

February 1997

(C) John Paul Blakeslee, MCMXCVII. All rights reserved.

The author hereby grants to MIT permission to reproduce and distribute publicly paper and electronic copies of this thesis document in whole or in part, and to grant others the right to do so.

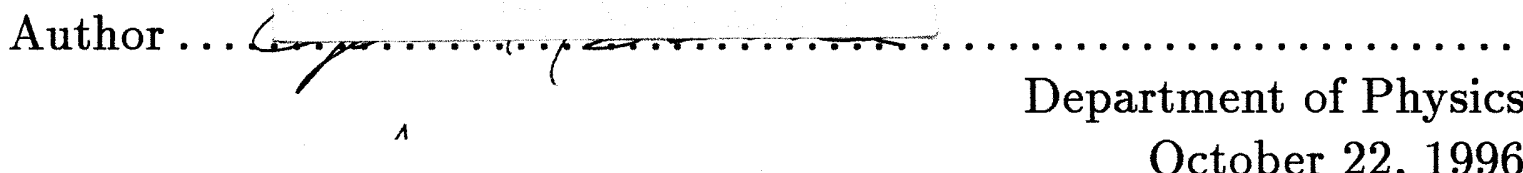

Certified by $\ldots y . \cdots \cdots \cdots \cdots \cdots \cdots$

John L. Tonry Professor of Physics Thesis Supervisor

Accepted by......

George F. Koster Chairman, Departmental Committee on Graduate Students wrsecmescremsmon of rethos:

FEB 121997 


\title{
The Globular Cluster Luminosity Functions of Brightest Cluster Galaxies
}

by

\author{
John Paul Blakeslee
}

Submitted to the Department of Physics

on October 22, 1996, in partial fulfillment of the

requirements for the degree of

Doctor of Philosophy

\section{Abstract}

A new technique for studying globular cluster populations around relatively distant galaxies is developed and applied to a sample of 23 galaxies in 19 Abell clusters. The technique is based on the surface brightness fluctuations method of determining galaxy distances. The galaxies in this sample range in redshift from 5000 to 10,000 $\mathrm{km} \mathrm{s}^{-1}$, and were selected from the Lauer \& Postman (1994) survey.

The analysis assumes that the mean magnitude of the globular cluster luminosity functions (GCLFs) in galaxies near the centers of rich clusters does not vary significantly; this assumption is scrutinized before proceeding with the Abell cluster study. The zero point of the GCLF mean is set with respect to Virgo, and is therefore independent of the Hubble parameter. The specific frequency $S_{N}$ of globular clusters within a metric radius of $32 h^{-1} \mathrm{Mpc}$ is found to correlate strongly with the velocity dispersion of the galaxies in the cluster, the cluster X-ray temperature and luminosity (especially "local" X-ray luminosity), and with the number of bright neighboring galaxies. $S_{N}$ correlates less strongly with galaxy profile, and only marginally with galaxy luminosity and overall cluster richness. It does not correlate with cluster morphology class. Within a cluster, galaxies at smaller projected distances from the $\mathrm{X}$-ray center have higher values of $S_{N}$.

Together with the relative constancy of BCG luminosity, these results suggest a scenario in which globular clusters form in proportionate numbers to the available mass, but central galaxy luminosity "saturates" at a maximum threshold, resulting in higher $S_{N}$ values for central galaxies in denser clusters. As a byproduct of the analysis, the Gaussian width $\sigma$ of the GCLF is measured. In the cosmic microwave background frame, the mean GCLF width for this sample is $\langle\sigma\rangle=1.43 \mathrm{mag}$, virtually identical to the M87 HST value. This provides a self-consistency check on the assumption of a constant GCLF mean magnitude.

Thesis Supervisor: John L. Tonry

Title: Professor of Physics 


\section{Acknowledgments}

There are many people whom I wish to thank for help, advice, and encouragement during my time at MIT. Following are just some of the people to whom I owe gratitude; I apologize to those inadvertently left out, and I thank them as well.

First off, I thank my advisor John Tonry. In an environment like that of MIT, it was truly refreshing to find someone like John. Nothing wrong with his ego, mind you, but what interests John is doing good and innovative science. He's about as clever as anyone you'd ever want to meet (more clever people may exist, but you wouldn't want to meet them), yet he isn't (much) concerned about impressing others with his cleverness. I am repeatedly amazed by his keen insights and scientific intuition, and I have learned an enormous amount from him over the past five years. My scientific writing skills have also improved greatly as a result of his advice and example (but don't get your hopes too high, gentle reader). Best of all, he truly cares about people and is not one for intimidation tactics, although sometimes he wishes he didn't care because he could get more work done that way. There's still a lot of good left in his bones as sets off for warmer climes, like Gary Cooper in High Noon.

I thank Paul Schechter for his example of scientific integrity and his comments on my thesis and other work through the past five years. The knowledge that Paul would be reading the thesis kept me striving for accuracy, honesty, and clarity; I hope he will continue in the future to provide feedback on my work. I also thank Earl Lomon for several comments that improved the quality of the thesis.

The thesis observations were carried out at MDM Observatory; I thank Bob Barr and the rest of the MDM staff for their excellent work in keeping the Observatory running and answering late night phone calls, as well as for being such good people. I really enjoyed (most of) my many nights and days at MDM.

I thank Matt Bershady and Alan Dressler for conversations, recommendations, and inspiration, Christine Jones-Forman for sending me the extremely valuable cluster $\mathrm{X}$-ray information, despite the fact that she was swamped with other things, Marc Postman and Tod Lauer for tabular data on the Lauer-Postman BCG sample, and 
Guy Worthey for redshifted model stellar populations calculations. I also thank Keith Ashman for many helpful email discussions.

I thank Chris Naylor, former secretary extraordinaire of MIT astrophysics. Chris may forget all about doing something vitally important that "must be done immediately", but it was worth it just to have him around. Thanks for everything, Chris, and good luck in your new job.

As all recent MIT physics graduate students, I owe an enormous debt of gratitude to Peggy Berkovitz. Too bad the great "they" have sequestered her away, but I'm sure Peggy will still find ways to dispense her wisdom to the hordes of hapless and confused graduate students who stumble into MIT each year.

A host of grad students, past and present, have been my companions on the journey, and I thank them for that. In particular, I thank my former officemates Ed Ajhar and Mark Metzger for help and advice on innumerable occasions and a variety of topics ranging from observing proposals to computers to apartments and much more. I don't know how I could have made it without both of them. I also thank for their help Max Avruch, Dave Buote, Charlie Collins, Jim Frederic, Eric Gaidos, Debbie Becker Haarsma, Lam Hui, Charlie Katz, Bob Rutledge, and Uros Seljak.

Pam and I are both extremely glad to have had friends like Jim \& Tonya Frederic and Charlie \& Kara Collins (as well as Ethan F. and Michael C., and little Margaret, though we haven't yet been formally introduced). You have all made our time at MIT much more bearable.

The best thing about MIT is the Tech Catholic Community; I thank all the people who make up that Community, especially the "local hierarchy" and the Sunday coffee \& donuts crowd. I thank the good Lord for bringing me to TCC, as well as for everything else, most especially the personal growth achieved during my MIT years.

I thank Michael Crescimanno for advice on things as disparate as mathematical physics and the quest for meaning in life. I have learned a great deal from him. Of course, I also thank Mary-K for marrying Mike despite all his advice-giving, and I thank her as well for her friendship towards Pam and me. Little Nicholas has a great pair of parents. 
Aimee \& Stefano Schiaffino were nothing short of a godsend for us over the past several years; we couldn't imagine two more generous people, and we thank them sincerely. We are happy to know Enrico will grow up to be just like both of them (even if he does look more like Stefano). Mike \& Christy Klug and Bill \& Diane Daughton have also been generous in so many ways, and Pam \& I thank them for everything. Of course, we also thank Sam \& David K. and Ashlyn, Tess, \& Bridget D. for inspiration.

I thank Justin Dougherty, Shin Kurokawa, Marty Minder, and Natalie Hawkins for sustained friendship through the years. Maybe some of Shin's cultural sensibilities will yet rub off on me; Natalie has been a great and loyal emailer; I was lucky to have had a best man with shoulders as broad as Marty's; whenever I talk to Justin I somehow recall what it means to be witty, as if all these hours in front of a workstation had never come to pass. I also thank Jim Doll for recent heroics at Justin's wedding, but wish to remind him that there are more things in Heaven and Earth than are dreamt of in his philosophy.

The various and scattered members of my family have given me much help and support (other things, too, but I thank them for their help and support), and so I thank my mother above, my father, sisters, brothers, nieces, and nephews. In particular, I thank my sister Ginny for escorting Pam \& I around in the sweltering heat of Pasadena so we could find a place to live.

Most of all, I wish to thank my sweet and wonderful wife Pam for her boundless love and support. Without Pam, I could never have done this thesis, for the the stars in the heavens above would not shine for me without her, and the galaxies would all just blink out. Though the affairs of two (soon to be three) little people may not amount to a hill of beans in this crazy world, as long as we can keep seeking the peaks and pits of life together, I'll be happy. 


\section{Contents}

1 Introduction 14

1.1 Background . . . . . . . . . . . . . . . . . 14

1.2 Historical Context $\ldots \ldots \ldots \ldots \ldots \ldots$

1.3 Scientific Motivation . . . . . . . . . . . . . . 17

1.4 Overview . . . . . . . . . . . . . . . . . . . 19

1.5 References . . . . . . . . . . . . . . . . . 21

2 Globular Clusters in the Coma Supergiant Ellipticals 23

2.1 Background . . . . . . . . . . . . . . . . . 24

2.2 Observations and Reductions $\ldots \ldots \ldots \ldots \ldots$

2.3 Results . . . . . . . . . . . . . . . . . . . 28

2.3.1 Evaluating the Various Variances . . . . . . . . . . 28

2.3.2 Counting the Bright End . . . . . . . . . . . . 32

2.3.3 Fluctuation Measurements . . . . . . . . . . . . . 38

2.3.4 Constraints on Coma GCS Properties . . . . . . . . . . . . 39

2.3.5 Results for Virgo Galaxies . . . . . . . . . . . . . 46

2.4 Discussion . . . . . . . . . . . . . . . . 50

2.4.1 The GCLF Parameters . . . . . . . . . . . . . . 50

2.4.2 Specific Frequencies and cD Galaxies . . . . . . . . . 51

2.5 Summary and Conclusions . . . . . . . . . . . . . 53

2.6 References . . . . . . . . . . . . . . . . . 54

3 The GCLF Next Door: Fornax and Virgo 56 


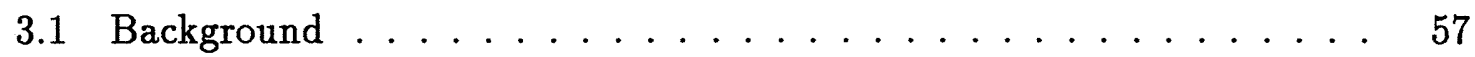

3.2 Observations and Reductions $\ldots \ldots \ldots \ldots \ldots$

3.3 Results . . . . . . . . . . . . . . . . . . 6 61

3.3.1 The GCLF in Fornax . . . . . . . . . . . . . . . 61

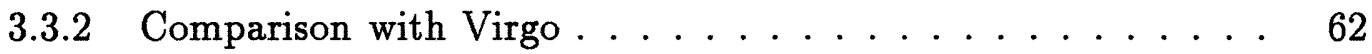

3.3.3 Does $M^{0}$ Depend on Environment? . . . . . . . . . . 64

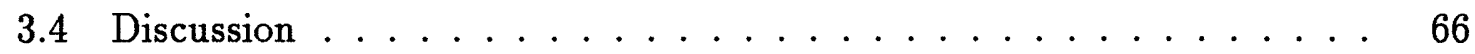

3.5 References . . . . . . . . . . . . . . . . . . . 69

4 Globular Clusters in Abell Clusters: Sample Selection, Observations, and Reductions

4.1 The BCG Sample . . . . . . . . . . . . . . . . 72

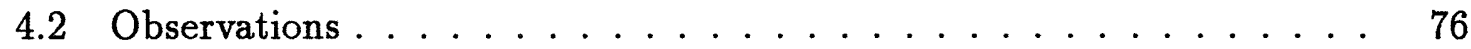

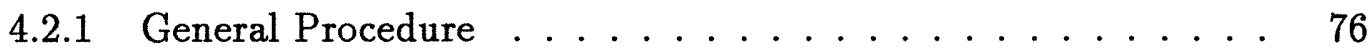

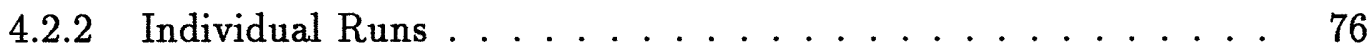

4.3 Image Reductions . . . . . . . . . . . . . . . . . 78

$4.3 .1 \quad$ Initial Processing $\ldots \ldots \ldots \ldots \ldots \ldots \ldots$

4.3.2 Photometric Calibration . . . . . . . . . . . . 79

4.3.3 The Galaxy Images . . . . . . . . . . . . . . . . . 81

4.3.3.1 Image Quality . . . . . . . . . . . 81

4.3.3.2 Final Data Set . . . . . . . . . . . . 83

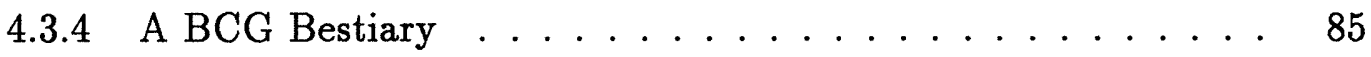

4.3.4.1 Contour Plots . . . . . . . . . . . . 85

4.3.4.2 Comments on Individual Galaxies . . . . . . . . . 98

4.3.5 Galaxy Modeling and Subtraction . . . . . . . . . . 100

4.4 Point Source and Fluctuation Reductions . . . . . . . . . . . . . 102

4.4.1 Finding Things with DoPhot . . . . . . . . . . 102

4.4 .2 Completeness Tests . . . . . . . . . . . . . . . . . 103

4.4.3 Measuring the Power Spectra . . . . . . . . . . 108

4.5 References . . . . . . . . . . . . . . . . . . . 115 
5 Globular Clusters in Abell Clusters: Properties and Implications 117

5.1 Analysis . . . . . . . . . . . . . . . . . . 117

5.1.1 Point Source Radial Distributions and Backgrounds . . . . . 117

5.1.2 Globular Cluster and Background Variances . . . . . . . 132

5.1 .3 Estimating $m^{0} \ldots \ldots \ldots \ldots \ldots \ldots \ldots \ldots$

5.1 .4 Constraining $S_{N}$ and $\sigma \ldots \ldots \ldots \ldots \ldots \ldots \ldots$

5.2 Results . . . . . . . . . . . . . . . . . . 137

5.2 .1 GCLF Widths . . . . . . . . . . . . . . 137

$5.2 .2 \quad$ Specific Frequencies . . . . . . . . . . . . . . . . 141

5.2 .3 Correlations . . . . . . . . . . . . . . . . 146

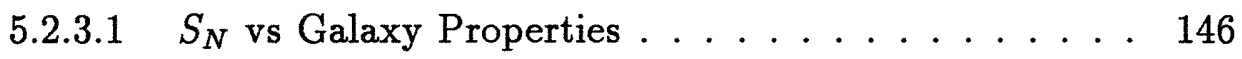

5.2.3.2 $S_{N}$ vs Cluster Properties . . . . . . . . 147

5.2.4 The Abell Cluster Inertial Frame . . . . . . . . . . . 164

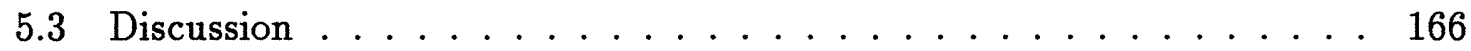

5.3.1 Are High- $S_{N}$ BCGs Special? $\ldots \ldots \ldots \ldots \ldots$

5.3.2 How Do the Models Fare? . . . . . . . . . . . . . . 168

5.3 .3 A Unified Model . . . . . . . . . . . . . . . . . . 174

5.4 References . . . . . . . . . . . . . . . . . 181

$\begin{array}{lll}6 & \text { Summary and Conclusions } & 186\end{array}$

$\begin{array}{ll}\text { A Probability Contours for } S_{N} \text { and } \sigma & 190\end{array}$ 


\section{List of Figures}

2-1 Ratio of GC to SBF Variance vs. Distance Modulus . . . . . . . . 33

2-2 Radial Number Density Distributions for Coma Galaxies . . . . . . 35

2-3 Radial Number Density Distributions for Virgo Galaxies . . . . . . 36

2-4 Comparison of $S_{N}$ vs. $\sigma$ Derived via Counts and Fluctuations $\ldots 40$

$2-5$ Confidence Contours on $S_{N}$ and $\sigma$ for NGC $4874 \ldots \ldots 41$

$2-6$ Confidence Contours on $S_{N}$ and $\sigma$ for NGC $4489 \ldots \ldots 42$

2-7 Confidence Contours on $S_{N}$ and $\sigma$ for NGC $4472 \ldots \ldots \ldots$

2-8 Confidence Contours on $S_{N}$ and $\sigma$ for NGC $4486 \ldots \ldots$

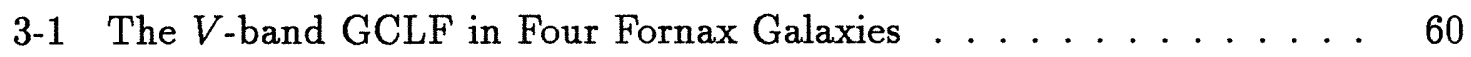

$3-2$ The $R$-band GCLF in Five Virgo Galaxies . . . . . . . . 63

3-3 $\quad M_{V}^{0}$ vs. Local Velocity Dispersion . . . . . . . . . . . . 65

3-4 Illustration of Variable Creation/Destruction Mechanisms . . . . . 67

4-1 Standard Star Photometry . . . . . . . . . . . . . . 80

4-2 A262-1 Isophotal Contours . . . . . . . . . . . . . . 86

4-3 A347-1 Isophotal Contours . . . . . . . . . . . . . 86

4-4 A397-1 Isophotal Contours . . . . . . . . . . . . . . . . 87

4-5 A569-1 Isophotal Contours . . . . . . . . . . . . . 87

4-6 A539-1 Isophotal Contours . . . . . . . . . . . . . . . 88

4-7 A539-2 Isophotal Contours . . . . . . . . . . . . . . . 88

4-8 A634-1 Isophotal Contours . . . . . . . . . . . . . . . . . . 89

4-9 A779-1 Isophotal Contours . . . . . . . . . . . . . . . 89

4-10 A999-1 Isophotal Contours . . . . . . . . . . . . . . . . 90 
4-11 A1016-1 Isophotal Contours . . . . . . . . . . . . . . 90

4-12 A1177-1 Isophotal Contours . . . . . . . . . . . . . . 91

4-13 A1185-1 Isophotal Contours . . . . . . . . . . . . . . . . 91

4-14 A1314-1 Isophotal Contours . . . . . . . . . . . . . . . . . 92

4-15 A1367-1 Isophotal Contours . . . . . . . . . . . . . . . 92

4-16 A1656-1 Isophotal Contours . . . . . . . . . . . . . . . . 93

4-17 A1656-2 Isophotal Contours . . . . . . . . . . . . . . . . 93

4-18 A1656-3 Isophotal Contours . . . . . . . . . . . . . . . . . . 94

4-19 A2162-1 Isophotal Contours . . . . . . . . . . . . . . . 94

4-20 A2197-1 Isophotal Contours . . . . . . . . . . . . . . 95

4-21 A2197-2 Isophotal Contours . . . . . . . . . . . . . 95

4-22 A2199-1 Isophotal Contours . . . . . . . . . . . . . . . 96

4-23 A2634-1 Isophotal Contours . . . . . . . . . . . . . . 96

4-24 A2666-1 Isophotal Contours . . . . . . . . . . . . . . . 97

4-25 A539-2 Galaxy Model . . . . . . . . . . . . . . . . . . . 101

4-26 A2634-1 Galaxy Model . . . . . . . . . . . . . . . . . . 102

4-27 A347-1 Power Spectrum . . . . . . . . . . . . . . . 110

4-28 A2634-1 Power Spectrum . . . . . . . . . . . . . . . . . 111

4-29 A2199-1 Power Spectrum . . . . . . . . . . . . . . . . 112

4-30 A2197-2 Power Spectrum . . . . . . . . . . . . . . . . 113

4-31 A1185-1 Power Spectrum . . . . . . . . . . . . . . . . . 114

5-1 A262-1 Radial Point Source Distribution . . . . . . . . . . . 121

5-2 A347-1 Radial Point Source Distribution . . . . . . . . . . 122

5-3 A397-1 Radial Point Source Distribution . . . . . . . . . . . 122

5-4 A569-1 Radial Point Source Distribution . . . . . . . . . . . . 123

5-5 A539-1 Radial Point Source Distribution . . . . . . . . . . 123

5-6 A539-2 Radial Point Source Distribution . . . . . . . . . . . . 124

5-7 A634-1 Radial Point Source Distribution . . . . . . . . . . . . 124

5-8 A779-1 Radial Point Source Distribution . . . . . . . . . . . 125 
5-9 A999-1 Radial Point Source Distribution . . . . . . . . . . . 125

5-10 A1016-1 Radial Point Source Distribution . . . . . . . . . 126

5-11 A1177-1 Radial Point Source Distribution . . . . . . . . . 126

5-12 A1185-1 Radial Point Source Distribution . . . . . . . . . 127

5-13 A1314-1 Radial Point Source Distribution . . . . . . . . . . 127

5-14 A1367-1 Radial Point Source Distribution . . . . . . . . . 128

5-15 A1656-3 Radial Point Source Distribution . . . . . . . . . . 128

5-16 A2162-1 Radial Point Source Distribution . . . . . . . . . . 129

5-17 A2197-1 Radial Point Source Distribution . . . . . . . . . 129

5-18 A2197-2 Radial Point Source Distribution . . . . . . . . . 130

5-19 A2199-1 Radial Point Source Distribution . . . . . . . . 130

5-20 A2634-1 Radial Point Source Distribution . . . . . . . . . . 131

5-21 A2666-1 Radial Point Source Distribution . . . . . . . . . 131

5-22 Maximum Likelihood Background Luminosity Functions . . . . . . 134

5-23 Best-fit $\sigma$ vs. Uncertainty . . . . . . . . . . . . . . 139

$5-24 S_{N}$ vs. Galaxy Luminosity . . . . . . . . . . . 148

$5-25 S_{N}$ vs. Galaxy Extent . . . . . . . . . . . . . 149

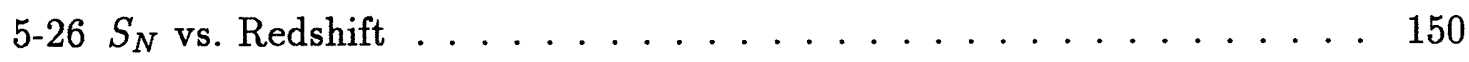

5-27 $S_{N}$ vs. Abell Cluster Central Velocity Dispersion . . . . . . . 153

$5-28 S_{N}$ vs. Abell Cluster Asymptotic Velocity Dispersion $\ldots \ldots \ldots 153$

$5-29 S_{N}$ vs. Abell Counts $\ldots \ldots \ldots \ldots \ldots$

5-30 $S_{N}$ vs. Number of Neighboring Galaxies $\ldots \ldots \ldots \ldots \ldots$

$5-31 S_{N}$ vs. Cluster Morphological Class $\ldots \ldots \ldots \ldots \ldots$

$5-32 S_{N}$ vs. Cluster X-ray Temperature . . . . . . . . . 161

5-33 $S_{N}$ vs. Cluster X-ray Luminosity . . . . . . . . . 161

5-34 Excess GC Number vs. Projected Mass Density . . . . . . . . . . 162

5-35 Excess GC Number vs. Projected Mass Density, $r_{c} \equiv 30 h^{-1} \mathrm{kpc} \ldots 162$

$5-36 S_{N}$ vs. Local X-ray Luminosity $\ldots \ldots \ldots \ldots$

5-37 Cluster Velocity Dispersion vs. X-ray Temperature . . . . . . . 163

5-38 Best-fit ACI Frame $\sigma$ vs. Uncertainty . . . . . . . . . . 165 
5-39 BCG Luminosity vs. Cluster Dispersion . . . . . . . . . . . . 177

5-40 Excess GC Number vs. Cluster Dispersion . . . . . . . . . . 177

$5-41$ BCG $\alpha$ vs. Cluster Dispersion . . . . . . . . . . . 178

A-1 A262-1 Probability Contours . . . . . . . . . . . . . . . 191

A-2 A347-1 Probability Contours . . . . . . . . . . . . . . . 192

A-3 A347-1 Probability Contours . . . . . . . . . . . . . . . 193

A-4 A397-1 Probability Contours . . . . . . . . . . . . . . . 194

A-5 A539-1 Probability Contours . . . . . . . . . . . . 195

A-6 A539-2 Probability Contours . . . . . . . . . . . . . 196

A-7 A569-1 Probability Contours . . . . . . . . . . . . . 197

A-8 A569-1 Probability Contours . . . . . . . . . . . . . . . 198

A-9 A634-1 Probability Contours . . . . . . . . . . . . . . . . 199

A-10 A779-1 Probability Contours . . . . . . . . . . . . . . 200

A-11 A999-1 Probability Contours . . . . . . . . . . . . . . . . 201

A-12 A1016-1 Probability Contours . . . . . . . . . . . . . 202

A-13 A1177-1 Probability Contours . . . . . . . . . . . . . . 203

A-14 A1185-1 Probability Contours . . . . . . . . . . . . . . 204

A-15 A1314-1 Probability Contours . . . . . . . . . . . . . 205

A-16 A1367-1 Probability Contours . . . . . . . . . . . . 206

A-17 A1656-3 Probability Contours . . . . . . . . . . . . . . 207

A-18 A2162-1 Probability Contours . . . . . . . . . . . . . . 208

A-19 A2197-1 Probability Contours . . . . . . . . . . . . . 209

A-20 A2197-2 Probability Contours . . . . . . . . . . . . 210

A-21 A2199-1 Probability Contours . . . . . . . . . . . . . . 211

A-22 A2634-1 Probability Contours . . . . . . . . . . . . . . 212

A-23 A2666-1 Probability Contours . . . . . . . . . . . . . 213 


\section{List of Tables}

2.1 Fluctuation Measurements and Counts for Coma Galaxies . . . . . 39

2.2 GCS Properties for Coma Galaxies . . . . . . . . . . . . . . . 43

2.3 Fluctuation Measurements and Counts for Virgo Galaxies . . . . . . 46

3.1 Fornax GCLF Parmeters . . . . . . . . . . . . . . 62

4.1 The BCG Sample . . . . . . . . . . . . . . . . . 74

4.2 Abell Cluster Information . . . . . . . . . . . . . 75

4.3 Observing Runs . . . . . . . . . . . . . . . . . 77

4.4 Landolt Photometric Standard Fields . . . . . . . . . . . . 79

4.5 Galaxy Observations . . . . . . . . . . . . . . . . 84

4.6 DoPhot Completeness Tests . . . . . . . . . . . . . . . 105

5.1 Point Source Counts and Variance Measurements . . . . . . . . 118

5.2 Background Galaxy Count Normalizations . . . . . . . . . 135

5.3 Metric Specific Frequencies and GCLF Widths in CMB Frame . . . 142

5.4 Comparison of $S_{N}^{f l u}$ and $S_{N}^{\text {cnt }}$ for $\sigma=1.40 \mathrm{mag} \ldots \ldots \ldots \ldots$

5.5 Neighboring Galaxy Counts . . . . . . . . . . . . . . 155

5.6 Metric Specific Frequencies and GCLF Widths in ACI Frame . . . . 165 


\section{Chapter 1}

\section{Introduction}

\subsection{Background}

This thesis grew out of a spontaneous experiment during a night of good seeing in April 1993 at MDM Observatory. John Tonry was working on MDM's new Tektronix CCD camera, while I was imaging for the surface brightness fluctuations (SBF) distance survey (Tonry et al. 1997) using the Loral CCD camera known as Wilbur (Metzger, Tonry, \& Luppino 1993). Mark Metzger had arrived early for his observing run, so he was pacing sleeplessly around, helping John out and keeping an eye on the spiders which had unaccountably proliferated in the observing room that spring. Informed of the steady image quality, John suggested I turn the telescope towards the two big Coma cluster ellipticals, NGC 4874 and NGC 4889, in order to see if it were possible to learn something about the relative sizes of their globular cluster systems by differentially comparing the amplitudes of their surface brightness variances. Since these two galaxies are at the same distance and position in the sky, any significant difference in the SBF amplitude would be due to a difference in the number density of globular clusters. Thus, in this case, it would be straightforward to adapt the analysis methods used in the SBF survey to the study of globular cluster systems. (A globular cluster system [GCS] is defined as the entire ensemble, or population, of globular clusters [GCs] which occupy the halo region of galaxy.)

Intrigued by the idea, I took the data, then left it alone for over a year before reducing it and discovering that the idea worked quite well. Moreover, we had inte- 
grated long enough that the brightest members of the GCSs were clearly visible above the background of unresolved sources. We found that we could use the counts of these bright GCs, together with our measurements of the amount of surface brightness variance from GCs below the limit of direct detection, to constrain the globular cluster luminosity functions (GCLFs) in these galaxies. We were also able to constrain the total numbers of GCs much more accurately than had been done previously (Harris 1987; Thompson \& Valdes 1987).

Eventually it was decided that I should apply these methods to study more GCSs around bright central galaxies in clusters, the primary project in this thesis. The following sections place the thesis in an historical context, discuss the scientific motivations for having undertaken it, and give an outline of what is to follow.

\subsection{Historical Context}

A number of good reviews exist on the subject of extragalactic globular cluster systems (Harris \& Racine 1979; Hanes 1980; Harris 1988, 1991, 1993). This section mentions only a few of the highlights in the history of the field, primarily culled from these reviews. The interested reader is encouraged to consult both the reviews and the original works for more information.

As pointed out by Harris (1988), Harlow Shapley (1918) was the first to study a system of globular clusters as an entity unto itself when he used the three dimensional spatial distribution of the Milky Way GCs to infer the distance to the Galactic center. The study of GCSs around external galaxies began with Hubble (1932), who "provisionally identified as globular clusters" the relatively bright, slightly extended objects in the halo of M31. Progress was slow, however, and it was more than twenty years before Baum (1955) identified the brightest members of the extremely rich GCS which surrounds M87, the central giant elliptical in Virgo. He attempted to derive a crude distance to Virgo, but it was a significant underestimate due to the fact that the GCLF extends to brighter magnitudes in M87 than in the comparatively small systems of M31 and the Milky Way. 
Kron \& Mayall (1960) made a landmark study of Local Group GCSs, deriving distance moduli to M31 and the Magellanic Clouds based on the means of their luminosity functions. Later, more detailed photographic studies of the color and luminosity distributions of the M87 system were carried out, most notably by Racine (1968a,b). The GCSs of three Fornax galaxies were detected by Dawe \& Dickens (1976), and the first GCS significantly more distant than Virgo was observed by Smith \& Weedman (1976) around the Hyrda cD NGC 3311. However, the GCS of any Virgo galaxy other than M87 remained unknown until the thesis work of Hanes (1977a,b), who published single-color photometric photometry for objects in the fields of 20 Virgo galaxies, finding significant GC populations around many of them. $\mathrm{He}$ added the GCLFs of the five largest of these make a composite GCLF, then derived the distance to Virgo through a comparison with the Milky Way GCLF. The result was still uncertain, however, because the mean, or turnover, of the Virgo GCLF had not been reached. Significant further progress awaited an advance in astronomical imaging.

It has become something of a cliche to remark on the "advent of CCDs" and the "revolution" they engendered in any given area of astronomy. Yet cliche or not, the advent of CCDs has certainly engendered a revolution in the field of extragalactic globular cluster research. The first shots were fired in the mid-1980s with the work of van den Bergh et al. (1985), who predictably used the new tool to study the M87 GCS, finally reaching the point at which its luminosity function begins to turn over. CCD studies of GCSs around ellipticals in Leo (Pritchet \& van den Bergh 1985), Coma (Harris 1987; Thompson \& Valdes 1987), and Virgo (Cohen 1988) followed soon after; the number of such studies has increased dramatically through the 1990s.

The most recent advances in the field have come about as a result of the revolutionary image quality of HST. With HST, it has become possible to image two magnitudes beyond the turnover in the all-important M87 system (Whitmore et al. 1995), see to unprecedented depths along the Coma GCLF (Baum et al. 1995), and study a larger number of smaller GCSs in detail (Forbes et al. 1996, which also contains some review material). 
Besides the boon to GCS research, CCDs allowed for the development of the SBF method of distance determination (Tonry \& Schneider 1988; Tonry, Ajhar, \& Luppino 1990; Tonry et al. 1997; but see also Shopbell et al. 1993, who showed that it could be done, with considerable effort, on photographic plates). The SBF method measures the seeing-convolved variance, or "fluctuations," produced by the Poisson statistics of the stars in an early-type galaxy. The amplitude of the fluctuations decreases with the square of the distance to the galaxy, and when divided by the galaxy's mean surface brightness, yields the luminosity-weighted average flux of the stars within the galaxy. This flux, usually referred to in terms of magnitudes and called $\bar{m}$, gives the distance to the galaxy after proper calibration.

In the present work, the SBF image analysis methods are used to measure the fluctuations produced by globular clusters surrounding large galaxies. The fluctuations provide information on the number and luminosity distribution of GCs below the limit of direct photometric detection. Like CCDs and HST, the application of these analysis techniques to the the field of extragalactic GC research constitutes a "revolution," though on a humbler scale. This thesis chronicles the initial skirmishes.

\subsection{Scientific Motivation}

As alluded to above, GC populations around galaxies can be studied both through their brightest members, which appear as faint point sources, and also through the surface brightness variance produced in the image by the remainder of the population (those too faint to be detected as point sources). This variance is a nuisance which must be subtracted from the SBF amplitude if one's goal is to derive an accurate distance, as in the SBF survey. However, it can also be used as a powerful a probe of the GC population. In bands bluer than the usual SBF survey $I$, the GC component dominates the total SBF amplitude in elliptical galaxies. By using all of the available information, counts of the brightest GCs as well as the variance from the rest of the GCs, we can constrain the luminosity function of the population and determine the total GC population much more accurately, and to much larger distances. 
The number of GCs per unit galaxy luminosity is known as the "specific frequency (or number) of globular clusters," abbreviated $S_{N}$ (specifically, it is the number per $M_{V}=-15$ of galaxy luminosity). Some luminous central galaxies in clusters, M87 being the most famous, have huge GC populations, even when their large luminosity is taken into account. These central galaxies are called "high- $S_{N}$ systems" and sometimes described as having "excess" GC populations. When we first applied our new analysis method to the GC populations of the two central giants in Coma, at nearly six times the distance to Virgo, we found that the $\mathrm{cD}$ galaxy NGC 4874 was a high- $S_{N}$ galaxy while its neighbor NGC 4889 was not.

There has been considerable speculation on the origin of the high- $S_{N}$ systems, but until now, few observational constraints. One model postulates that primordial GC formation occurred more efficiently in the dense environments at the centers of galaxy clusters; thus, all cD galaxies formed as high- $S_{N}$ systems, but in some clusters the $\mathrm{cD}$ has diluted its GC population down to normal levels through mergers with other cluster galaxies (McLaughlin, Harris, \& Hanes 1994). $S_{N}$ would then be anticorrelated with the state of cluster dynamical evolution. A competing explanation (West et al. 1995) is that the excess GCs have been stripped over time from normal cluster galaxies and trace the cluster potential as a whole. They have little to do with any $\mathrm{cD}$ galaxy which may be located within the cluster core, but are mistakenly associated with it when the $\mathrm{cD}$ is very near the center of the cluster potential. Another suggestion (Djorgovski \& Santiago 1992) is that high- $S_{N}$ systems are not special; galaxies with extremely rich GCSs are simply at the high-luminosity end of a nonlinear relationship between total GC population and galaxy luminosity. Further possibilities are discussed in Chapter 5.

This thesis entails both the development of our new GC analysis technique and its application to a large, unbiased, sample of brightest galaxies in Abell clusters extending out to $10,000 \mathrm{~km} / \mathrm{s}$ The intent is to learn which central galaxies are high$S_{N}$ systems, which are not, and why. Published data sets have been inconclusive in distinguishing among the various models, but with our new analysis techniques and a systematic approach, we undertook this project in the hope of making significant 
progress towards an eventual understanding of the cores of galaxy clusters and of GCSs in general.

Along the way, we have learned more about variations in the GCLF, which has been used extensively as a distance indicator based upon the apparent universality of the turnover magnitude $M_{0}$ (e.g. Harris 1991 and references therein). The GCLF method has the potential to be enormously powerful with the aid of HST, but the universality of $M_{0}$ has yet to be firmly established (e.g. Blakeslee \& Tonry 1996), though it remains a good working hypothesis. In our initial Coma study, we extended by a factor of five the distance to which GCLF widths had been measured, and subsequent work reported in this thesis has gone further still.

\subsection{Overview}

This thesis consists of three basically independent, but closely related, projects. The first two of these (chapters 2 and 3) have been published previously, while the third, constituting the bulk of the thesis (chapters 4 and 5), is new. Each project is presented along with its own introductory material, detailed discussion, and references. Here, we provide an overview of the individual projects.

Chapter 2 presents the study of the two central giant ellipticals in Coma which first demonstrated that the SBF technique could be used to learn more about the GCSs of relatively distant galaxies than would otherwise be possible from the ground. This chapter also contains the mathematical background for the the remainder of the thesis; specifically, how the conversion from measured variance to specific globular cluster frequency is done and how the background variances are estimated. It also goes into some detail concerning the phenomenon of high- $S_{N}$ systems. The text of Chapter 2 is identical to that of the published paper (Blakeslee \& Tonry 1995), except for one extra figure, added for illustrative purposes, and its accompanying description.

Chapter 3 represents something of a digression in examining the globular cluster luminosity functions (GCLFs) of four bright galaxies in the Fornax cluster. Fornax is a nearby cluster in the southern sky which, due to its strong spatial concentration, is 
important for evaluating extragalactic distance estimators. The methods developed in the Coma study rely on the assumption that the mean magnitude $M^{0}$ of the GCLF is universal to within $\sim 0.25$ mag. In Fornax, $M^{0}$ is found to be universal at better than this level. A discussion of the GCLF in Virgo, based on a section I wrote for a different study (Ajhar, Blakeslee \& Tonry 1994) is then interjected, concluding that $M^{0}$ varies by less than this amount in Virgo as well. We then proceed to ask whether $M^{0}$, though constant within each of these clusters, differs between them, and more generally, whether it depends on the density of a galaxy's local environment. The evidence we find in this regard is a bit unsettling, but not conclusive, so we proceed, with some trepidation, to the study of the GCSs of brightest cluster galaxies which comprises the remainder of the thesis. Chapter 3 is virtually the same as the published paper (Blakeslee \& Tonry 1996) but includes some additional discussion, primarily the part about Virgo.

In Chapter 4, we present the data which make up the brightest cluster galaxy (BCG) sample and describe their reductions. This is a complete subsample of the northern portion of the Lauer \& Postman (1994) survey of 119 BCGs. Specifically, the sample includes all early-type BCGs in northern Abell clusters with $c z<$ $10000 \mathrm{~km} \mathrm{~s}^{-1}$, absolute $R$-band metric magnitude $M_{R}<-22.0+5 \log h_{75}$, and galactic latitude $|b|>15^{\circ}$. (The early-type and $M_{R}$ criteria exclude just two BCGs each.) It also includes three second brightest galaxies, and one third brightest, because these galaxies were similar to the respective BCGs in luminosity and extent. The final sample then comprises 23 galaxies in 19 Abell clusters (including the two Coma galaxies studied in Chapter 2).

Chapter 5 presents the results of the analysis of the GCSs in our BCG sample, including the radial distributions, specific frequencies, and GCLF widths. We also introduce something called the "metric specific frequency" which allows for unbiased comparison among the GCSs of galaxies at different distances. We find several correlations of this quantity with various properties of the galaxies and galaxy clusters; most notable are the correlations with cluster velocity dispersion and the local density of bright galaxies. The implications of these results for BCG formation and cluster 
evolution are discussed.

The final chapter summarizes the results of the thesis, attempts to synthesize what is currently known into a coherent picture of globular cluster systems, and points out some possible courses for future research.

\subsection{References}

Ajhar, E. A., Blakeslee, J. P., \& Tonry, J. L. 1994, AJ, 108, 2087

Baum, W. 1955, PASP, 67, 328

Baum, W. et al. 1995, AJ, 110, 2537

Blakeslee, J. P. \& Tonry, J. L. 1995, ApJ, 442, 579

Blakeslee, J. P. \& Tonry, J. L. 1996, ApJ, 465, L19

Cohen, J. G. 1988, AJ, 95, 682

Dawe, J. A. \& Dickens, R. J. 1976, Nature, 263, 395

Djorgovski, S. \& Santiago, B. X. 1992, ApJ, 391, L85

Forbes, D. A., Franx, M., Illingworth, G. D., \& Carollo, C. M. 1996, ApJ, 467, 126

Hanes, D. A. 1977a, MmRAS, 84, 45

Hanes, D. A. 1977b, MNRAS, 180, 309

Hanes, D. A. 1980, Globular Clusters, NATO Adv. Study Inst., ed. D. A. Hanes \& B. F. Madore (Cambridge: Cambridge Univ. Press), 213

Harris, W. E. 1987, ApJ, 315, L29

Harris, W. E. 1988, in Globular Cluster Systems in Galaxies, IAU Symp. No. 126, ed. J. Grindlay \& A. G. D. Philip (Dordrecht: Reidel), 237

Harris, W. E. 1991, ARA\&A, 29, 543

Harris, W. E. 1993, in The Globular Cluster-Galaxy Connection, ed. G. H. Smith \& J. P. Brodie (ASP Conf. Ser., 5), 472

Harris, W. E. \& Racine, R. 1979, ARA\&A, 17, 241

Hubble, E. 1932, ApJ, 76, 44

Kron, G. E. \& Maynall, N. U. 1960, AJ, 65, 581

McLaughlin, D. E., Harris, W. E., \& Hanes, D. A. 1994, ApJ, 422, 486 
Metzger, M. R., Tonry, J. L., \& Luppino, G. A. 1993, in Astronomical Data Analysis Software and Systems II, ed. R. J. Hanisch, R. J. V. Brissenden, \& J. Barnes (ASP Conference Series, 52), 300

Pritchet, C. J. \& van den Bergh, S. 1985, AJ, 90, 2027

Racine, R. 1968a, PASP, 80, 326

Racine, R. 1968b, JRASC, 62, 367

Shapley, H. 1918, ApJ, 48, 154

Shopbell, P. L., Bland-Hawthorn, J., \& Malin, D. F. 1993, AJ, 106, 1344

Smith, M. G. \& Weedman, D. W. 1976, ApJ, 205, 709

Thompson, L. A. \& Valdes, F. 1987, ApJ, 315, L35

Tonry, J. L. \& Schneider, D. P. 1988, AJ, 96, 807

Tonry, J. L., Ajhar, E. A., \& Luppino, G. A. 1990, AJ, 100, 1416

Tonry, J. L., Blakeslee, J. P., Ajhar, E. A., \& Dressler, A. 1997, ApJ, 475, in press

van den Bergh, S., Pritchet, C. J., \& Grillmair, C. G. 1985, AJ, 90, 595

West, M. J., Côté, P., Jones, C., Forman, W., \& Marzke, R. O. 1995, ApJ 435, L77

Whitmore, B. C., Sparks, W. B., Lucas, R. A., Macchetto, F. D., \& Biretta, J. A. 1995, ApJ, 454, L73 


\section{Chapter 2}

\section{Globular Clusters in the Coma Supergiant Ellipticals}

This chapter appeared in similar form as "Measurement of Globular Cluster Specific Frequencies and Luminosity Functions Widths in Coma", The Astrophysical Journal, Vol. 442, 579. [Some additional comments are given in square brackets.]

\section{Synopsis}

We use a variant of the surface brightness fluctuations (SBF) method to study the globular cluster populations around NGC 4874 and NGC 4889 in the $I$ band. The fluctuations due to globular clusters, a nuisance factor in the standard SBF technique, provide a means for investigating that portion of the globular cluster population which cannot be observed directly. At the distance of Coma, we can also directly count the number of clusters occupying the bright end of the globular cluster luminosity function (GCLF). The direct counting and fluctuation measurements together provide a constraint on the width of the GCLF, which in turn decreases the uncertainty in the inferred specific frequencies. We find the Gaussian widths of the luminosity functions to be $1.43 \pm 0.09 \mathrm{mag}$ and $1.37 \pm 0.13 \mathrm{mag}$ for NGC 4874 and NGC 4889 , respectively. Using these measured Gaussian widths, we derive specific frequencies ( $S_{N}$; number of globular clusters per $M_{V}=-15$ of galaxy luminosity) of $14.3 \pm 3.3$ for NGC 4874 and $6.9 \pm 1.8$ for NGC 4889 , confirming that NGC 4874 is a member 
of the class of anomalously high $S_{N}$ galaxies. By measuring the globular cluster fluctuations at several radii, we find that $S_{N}$ increases outward from the centers of both galaxies, indicating that the globular cluster systems are more extended than the halo light, in accord with previous studies of NGC 4874 and nearby ellipticals. We confirm the reliability of our technique for measuring GCLF widths by applying it to NGC 4472 (M49) and NGC 4486 (M87) in Virgo. A brief discussion of the universality of the GCLF width for large ellipticals and the high- $S_{N}$ anomaly of central galaxies is provided.

\subsection{Background}

The globular cluster systems (GCSs) of galaxies much more distant than the Virgo and Fornax clusters $(\sim 1300 \mathrm{~km} / \mathrm{s})$ have been exceedingly difficult targets for observation. Thompson \& Valdes (1987) and Harris (1987) detected the brightest members of the GCSs of the Coma giants NGC 4874 and NGC $4889(\sim 7000 \mathrm{~km} / \mathrm{s})$ as statistical excesses in the numbers of point sources around these galaxies. These studies probed deeply enough to see the brightest $\sim 5 \%$ of the globulars, from which the authors extrapolated according to the Virgo globular cluster luminosity function (GCLF) to estimate the sizes of the populations. Harris found that the number of globulars around NGC 4874 was about twice that around NGC 4889, and since NGC 4889 is $50 \%$ more luminous, the specific frequency $S_{N}$ (number of globulars per $M_{V}=-15$ of galaxy luminosity) was about 3 times greater for NGC 4874. Thus, NGC 4874 appeared to have the "high- $S_{N}$ anomaly" previously found in NGC 4486 (M87), NGC 1399, and NGC 3311, all large ellipticals located near the centers of galaxy clusters (see Harris [1991] for a review). The most distant detected GCS belongs to NGC 6166, the cD in Abell $2199(\sim 9000 \mathrm{~km} / \mathrm{s})$, studied by Pritchet \& Harris (1990) who were able to conclude that NGC 6166 was not a member of the class of anomalously high $S_{N}$ galaxies. From this conclusion and the fact that NGC 6166 is the center of a large cooling flow, these authors argued strongly against the hypothesis that the excess populations of globular clusters form continuously from cooling flows. 
The question of why some galaxies are overabundant in globular clusters remains unanswered, but the evidence clearly implicates these galaxies' special positions near the dynamical centers of clusters. Furthermore, McLaughlin, Harris, \& Hanes (1993) found that the GCS and $\mathrm{cD}$ envelope of M87 have structural similarities which hint at a common origin. They and others (Pritchet \& Harris 1990; Harris 1991) have argued that the accretion of matter from other cluster galaxies cannot result in higher specific frequencies, since halo stars and globular clusters would be added to the central giant in the proportions they occur in neighboring galaxies, serving only to dilute the GCS. On this basis, McLaughlin et al. suggested that NGC 6166 may in fact be a merger remnant, while high- $S_{N}$ galaxies like M87 were different from the start, forming their globular clusters with greater efficiency for reasons related to their preferred positions in cluster centers.

One of the main hindrances to a more complete understanding this phenomenon is the lack of data on high- $S_{N}$ systems other than M87 and NGC 1399, the only ones within $25 \mathrm{Mpc}$. In addition to its high $S_{N}$, M87 may have an anomalously broad GCLF. It is unknown whether this is a common feature of these systems [we find later in the thesis that this is not the case] because too few of them are near enough for direct observations to be done of a large enough percentage of the globular clusters that constraints can be placed on their GCLF parameters.

In this paper, we make use of the surface brightness fluctuations (SBF) technique to "observe" globular clusters below the limit of detection. The SBF produced by the Poisson statistics of stars have been used to measure accurate distances to early type galaxies and spiral bulges (Tonry \& Schneider 1988; Tonry, Ajhar, \& Luppino 1990; Tonry 1991). Here, we use the same technique to measure the fluctuations produced by globular clusters. Wing et al. (1995) have recently shown how readily these fluctuations are measured in M87. Coupled with our counts of the directly observable brightest globular clusters, our fluctuation measurements allow us to simultaneously constrain the GCLF widths and specific frequencies in NGC 4874 and NGC 4889. To test the accuracy of our results, we perform the same analysis on images of M49 (NGC 4472) and M87, galaxies with known GCLF widths. 


\subsection{Observations and Reductions}

We observed NGC 4874 and NGC 4889 in May 1993 with the $2.4 \mathrm{~m}$ telescope at the Michigan-Dartmouth-MIT Observatory (MDM) located on Kitt Peak. Nine exposures were taken of NGC 4874, five of $700 \mathrm{~s}$ duration and four of $500 \mathrm{~s}$; seven $700 \mathrm{~s}$ exposures were taken of NGC 4889. We used the camera known as "Wilbur" (Metzger, Tonry, \& Luppino 1993), whose Loral $2048^{2}$ CCD was binned to $1024^{2}$, yielding an image scale of $0.343 /$ pixel. The gain was set to $1.94 e^{-} /$ADU. All images were taken through MDM's $I$-band interference filter. NGC 4472 and NGC 4486 were observed at MDM in April 1993 with the identical setup. Four $500 \mathrm{~s}$ exposures were taken of each galaxy. Unlike the Coma observations, which were done specifically for the project described in this paper, the Virgo observations were obtained as part of the ongoing SBF distance survey.

Bias levels were subtracted from the individual frames using the overclock region of the CCD. The data were then flattened with twilight sky flats. (Wilbur has neglible dark current.) Final images were produced by registering the individual frames (we had "dithered" between exposures), removing cosmic rays and CCD defects, then adding the frames together. The final data set for this project consists of a $5500 \mathrm{~s}$ image of NGC 4874, a $4900 \mathrm{~s}$ image of NGC 4889, and $2000 \mathrm{~s}$ images of NGC 4472 and NGC 4486. The seeing in these four images are $0.83,0 " 88,0.97$, and 0.95 , respectively.

The photometry was calibrated to the Kron-Cousins $I$ band using Landolt (1983, 1992) standard stars. The Coma galaxy observations were not photometric, but we were able to calibrate their photometry through a comparison with shorter exposure images of these galaxies taken on photometric nights at MDM and reduced in the same manner. The Virgo galaxy images were taken under photometric conditions. Internal scatter among the standard stars and our comparisons with other galaxy photometry indicate that the photometric accuracy is about $2 \%$. We have corrected for Galactic extinction using the reddening values of Burstein and Heiles (1984).

The image processing is as described by Tonry, Ajhar, \& Luppino (1990) and 
elsewhere (e.g., Ajhar, Blakeslee, \& Tonry 1994). After removing all visible stars and background objects, we fit a series of ellipses with individually varying centers, ellipticities, and position angles to the mean galaxy surface brightness and interpolate to construct a smooth model, which we then subtract from the original image. The fields containing NGC 4874 and NGC 4889 are crowded with smaller Coma Cluster galaxies, some large enough to be fitted with our ellipse fitting program and subtracted. In the field of NGC 4874, we subtracted four small neighboring ellipticals with locations relative NGC 4874 of $76^{\prime \prime} \mathrm{W}, 10^{\prime \prime} \mathrm{S} ; 38^{\prime \prime} \mathrm{W}, 87^{\prime \prime} \mathrm{N} ; 106^{\prime \prime} \mathrm{E}, 127^{\prime \prime} \mathrm{N}$; and $12^{\prime \prime} \mathrm{E}, 3^{\prime \prime} \mathrm{S}$. In the field of NGC 4889 , we subtracted the elliptical neighbor located $49^{\prime \prime} \mathrm{W}, 38^{\prime \prime} \mathrm{N}$ of NGC 4889 . We adopted an iterative approach: fitting and subtracting the central galaxy, then fitting the smaller galaxies and subtracting the fits from the original image, refitting the central galaxy and subtracting from (another copy of) the original image, and finally, refitting and subtracting the smaller galaxies. After subtracting the galaxy fits, we fit the large scale residuals from the model subtraction and subtract these from the model-subtracted image. We call the final image the "residual image"; it contains foreground stars, neighboring and background galaxies, and the subtracted galaxy's brightest globular clusters on a very flat background.

A version of the automatic CCD photometry program DoPhot (Mateo \& Schechter 1989; Schechter, Mateo, \& Saha 1993) was used to find all objects above a signal-tonoise threshold of 4 and produce a list of their positions, magnitudes, and classification types. Our version of DoPhot has been modified to account for the extra noise present in the frame due to the subtracted galaxy. We use the difference between DoPhot's fit and aperture magnitudes to determine the zero point of DoPhot's magnitude scale. Placing DoPhot's relative magnitudes onto a standard scale adds an uncertainty of a few percent to the photometric zero point of the identified objects.

The SBF technique measures the psf-convolved variance ("fluctuations") in the residual image after all sources brighter than a cutoff magnitude $m_{c}(r)$ have been excised. The cutoff is a function of radius because it is defined as the magnitude of an object having a constant cutoff signal-to-noise (usually 4.5 ), which varies with the surface brightness of the subtracted galaxy. The contributions to the measured 
variance from globular clusters and faint galaxies below the cutoff magnitude are then subtracted according to a fitted combined luminosity function extrapolated from above the cutoff. The remaining psf-convolved variance, called the SBF, is assumed due to the Poisson statistics of the stars in the galaxy, yielding an average flux for the stellar population, and thence, a distance.

For this study, however, we subtract off the contributions from the stellar SBF and the faint galaxies, then assign the rest to the globular cluster population, yielding a surface density of globular clusters. A stepwise increasing $m_{c}$ is used, rather than a continuously varying one. The procedure we follow for measuring fluctuations has been described in detail by Tonry \& Schneider (1988), Tonry et al. (1990), and the distance measurement review of Jacoby et al. (1992). The last of these references provides a particularly thorough and up to date discussion. In the following section we describe how the contributions from galaxies and SBF are calculated and subtracted and the remaining variance converted to a number density of globular clusters.

\section{$2.3 \quad$ Results}

\subsubsection{Evaluating the Various Variances}

If all sources brighter than some limiting flux $f_{\text {lim }}$ have been removed from an image, then the pixel to pixel variance produced by the remaining part of a source population (before convolution with the psf) is

$$
\sigma_{p o p}^{2}=\int_{0}^{f_{l i m}} n(f) f^{2} d f
$$

where $n(f)$ is the number of sources per unit flux per pixel (Tonry \& Schneider 1988, equation [4]). Changing variables to magnitude $m$ via the relationship

$$
m=-2.5 \log (f)+m_{1}^{*}
$$

which defines $m_{1}^{*}$ as the magnitude of an object yielding one unit of flux per total integration time, and assuming a Gaussian form for the distribution of globular clusters 
in magnitude (Harris 1991)

$$
n(m)=\frac{N_{0}}{\sqrt{2 \pi} \sigma} e^{\frac{-\left(m-m^{0}\right)}{2 \sigma^{2}}}
$$

where $N_{0}$ represents the total number of globular clusters per pixel, we find

$$
\sigma_{G C S}^{2}=\frac{N_{0}}{\sqrt{2 \pi} \sigma} 10^{0.8 m_{1}^{*}} \int_{m_{c}}^{\infty} e^{\frac{-\left(m-m^{0}\right)^{2}}{2 \sigma^{2}}} e^{-0.8 \ln (10) m} d m
$$

Completing the square and integrating yields

$$
\sigma_{G C S}^{2}=\frac{1}{2} N_{0} 10^{0.8\left[m_{1}^{*}-m^{0}+0.4 \sigma^{2} \ln (10)\right]} \operatorname{erfc}\left(\frac{m_{c}-m^{0}+0.8 \sigma^{2} \ln (10)}{\sqrt{2} \sigma}\right)
$$

where $\operatorname{erfc}(x)$ is the complement of the error function. Thus, the variance due to globular clusters is directly proportional to their total surface density, as previously shown by Wing et al. (1995).

Before converting the measured $\sigma_{G C S}^{2}$ to a density of globular clusters, we need values for the GCLF parameters $\sigma$ and $m^{0}$. Large ellipticals typically have values for the width $\sigma$ near 1.4 mag (e.g. Harris 1991), with real variation from galaxy to galaxy (Harris et al. 1991; Secker and Harris 1993); however, no significant evidence exists for variation in the turnover magnitude $m^{0}$, which has been exploited by Harris and collaborators as a "standard candle" distance indicator (see Jacoby et al. [1992]). For this study, we provisionally fix $m^{0}$ and jointly constrain the quantities $N_{0}$ and $\sigma$ for each galaxy. The effects of varying $m^{0}$ will be examined in Section 4 . From Secker \& Harris (1993), we take the value $m_{V}^{0}=23.78 \pm 0.16$ for ellipticals in the Virgo Cluster core, from which we need to arrive at a value for the $I$-band turnover. The mean $V-I$ colors for the five largest globular cluster systems in the sample of Ajhar et al. (1994) range from 0.94 to 1.08 , with an overall median of 1.01. Couture, Harris, and Allwright $(1990,1991)$ report mean $V-I$ values of 0.99 and 1.09 for the globular cluster systems of NGC 4486 and NGC 4472, but suspect the presence of a 
systematic offset in the case of NGC 4472. Thus, let us settle on a value of

$$
m_{I}^{0}(\text { Virgo })=22.78 \pm 0.17
$$

for the $I$-band turnover magnitude in the Virgo core, and for a distance modulus of Coma relative Virgo of $\Delta(m-M)_{0}=3.72 \pm 0.15$ (Aaronson et al. 1986; Faber et al. 1989), we have

$$
m_{I}^{0}(C o m a)=26.50 \pm 0.22
$$

for the $I$-band turnover in Coma.

In order to determine the number density of globular clusters in this way, we must first subtract off the contributions to the fluctuations from the background galaxies and SBF. A good approximation for the magnitude distribution of the background galaxies is

$$
n(m)=B \times 10^{\gamma m}
$$

Tyson (1988) finds $\gamma \approx 0.34$ in the $I$ band, consistent with the value of 0.32 found by Lilly, Cowie, and Gardner (1991). We can normalize with respect to Tyson's counts by setting $B=p^{2} T_{n} 10^{-\gamma m_{T 1}}$, where $m_{T 1}$ is the magnitude at which Tyson would count 1 galaxy $/ \operatorname{arcsec}^{2}$ extrapolating his counts, $T_{n}$ is the ratio of our counts to Tyson's, and $p$ is the image scale in arcsec/pixel. An examination of Tyson's $I$-band counts reveals $m_{T 1} \approx 30.6$. Adopting this normalization and plugging into equation 2.1 , we find after integrating

$$
\sigma_{b g}^{2}=\frac{p^{2} T_{n}}{(0.8-\gamma) \ln (10)} 10^{0.8\left(m_{1}^{*}-m_{c}\right)-\gamma\left(30.6-m_{c}\right)}
$$

for the variance due to background galaxies. The fluctuations due to the globular clusters will dominate the background if the density of globulars substantially exceeds that of the galaxies just beyond the cutoff, as is the case in the halos of large ellipticals. See Wing et al. (1995) for a more detailed discussion.

The other source of contamination is the variance due to SBF, which is usually 
characterized in terms of the "fluctuation magnitude" $\bar{m}$, defined observationally as

$$
\bar{m}=-2.5 \log \left(\frac{\sigma_{S B F}^{2}}{\langle\text { gal }\rangle}\right)+m_{1}^{*}
$$

where $\langle\mathrm{gal}\rangle$ is the mean galaxy brightness in units of $e^{-} /$pixel over the region of interest. The mean observed value of $\bar{m}$ for 27 large ellipticals in Virgo is 29.60 (Tonry 1995) with an rms scatter of 0.22 due to stellar population differences and Virgo's spatial extent. This value of $\bar{m}$ agrees with the theoretical models of Worthey (1993), which predict $\bar{m}_{I}=29.46$ for $V-I=1.20$ and the Virgo distance modulus of $31.02 \pm 0.22$ proffered by Jacoby et al. (1992; assumes a distance of $0.77 \mathrm{Mpc}$ to M31). With the relative distance modulus of Coma adopted above, the expected value of $\bar{m}$ for Coma ellipticals is $33.32 \pm 0.27$.

It is informative to compare the globular cluster fluctuations with the SBF. Consider some region of the image encompassing $n_{p}$ pixels and with a mean intensity due to the central galaxy of $\langle$ gal $\rangle e^{-} /$pixel. The specific frequency in the region is $S_{N}=N_{0} n_{p} 10^{0.4\left[M_{I}+(V-I)+15\right]}$, where $N_{0}$ is the mean number per pixel of globular clusters in the region, $M_{I}$ is the absolute magnitude of the region, and $(V-I)$ is its color. Substituting $M_{I}=-2.5 \log \left(n_{p}\langle\right.$ gal $\left.\rangle\right)+m_{1}^{*}-D M$, where $D M$ is the distance modulus, and simplifying we find

$$
N_{0}=S_{N}\langle\text { gal }\rangle 10^{-0.4\left[m_{1}^{*}-D M+(V-I)+15\right]}
$$

which can be substituted into equation 2.5 to get

$$
\sigma_{G C S}^{2}=\frac{S_{N}}{2}\langle\text { gal }\rangle 10^{0.4\left[m_{1}^{*}-2 m^{0}+D M-(V-I)+0.8 \sigma^{2} \ln (10)-15\right]} \operatorname{erfc}(Z)
$$

where $Z$ represents the argument of the error function complement in equation 2.5 . We can write all the distance dependent quantities in terms of distance moduli relative to Virgo:

$$
\sigma_{G C S}^{2}=\frac{S_{N}}{2}\langle\text { gal }\rangle 10^{0.4\left[m_{1}^{*}-2 m_{\mathrm{Vir}}^{0}+D M_{\mathrm{Vir}}-\Delta D M_{\mathrm{Vir}}-(V-I)+0.8 \sigma^{2} \ln (10)-15\right]} \operatorname{erfc}(Z)
$$


Now, using the empirical $\bar{m}$ calibration of Tonry (1991) and the SBF distance to Virgo (Jacoby et al. 1992), we can write equation 2.10 as

$$
\sigma_{S B F}^{2}=\langle\text { gal }\rangle 10^{-0.4\left[26.03+3(V-I)+\Delta D M_{\mathrm{Vir}}-m_{1}^{*}\right]}
$$

Thus, the ratio of the two is

$$
\frac{\sigma_{G C S}^{2}}{\sigma_{S B F}^{2}}=\frac{S_{N}}{2} 10^{0.4\left[D M_{\mathrm{Vir}}-2 m_{\mathrm{Vir}}^{0}+2(V-I)+0.8 \sigma^{2} \ln (10)+11.03\right]} \operatorname{erfc}(Z)
$$

Substitution of appropriate values from above into the exponent yields

$$
\frac{\sigma_{G C S}^{2}}{\sigma_{S B F}^{2}} \approx 5 S_{N} \operatorname{erfc}\left(\frac{m_{c}-m^{0}+0.8 \sigma^{2} \ln (10)}{\sqrt{2} \sigma}\right)
$$

Figure 2-1 shows this ratio plotted as a function of distance modulus for $S_{N}=5$, $\sigma=1.4 \mathrm{mag}$ and a range in $m_{c}$. The dashed lines represent the distance moduli of Virgo and Coma. While the SBF dominate the fluctuations from globular clusters at the distance of Virgo with $m_{c} \gtrsim 22$, the reverse is true in Coma for $m_{c} \lesssim 25$. In bluer bands, the SBF are considerably fainter, but the background galaxy counts rise more steeply, so we have chosen to work in the $I$-band.

Glancing at the figure, one might deem it optimal to choose a very bright value for the cutoff magnitude; however, we are interested not only in measuring the globular cluster fluctuations, but in converting the measurements to specific frequencies. From the argument of the error function complement, it is apparent that brighter values of $m_{c}$ increase the sensitivity of the inferred specific frequency on the adopted value of the GCLF width $\sigma$. Moreover, we wish to utilize the bright end counts in conjunction with the fluctuations to constrain $\sigma$; thus, we choose to push $m_{c}$ as faint as reasonable.

\subsubsection{Counting the Bright End}

Before proceeding with the fluctuation analysis, we consider the sources brighter than the cutoff magnitude. In their study of the GCS of NGC 4874, Thompson \& Valdes (1987) discovered that the exclusion of nonstellar objects was essential for an accurate 


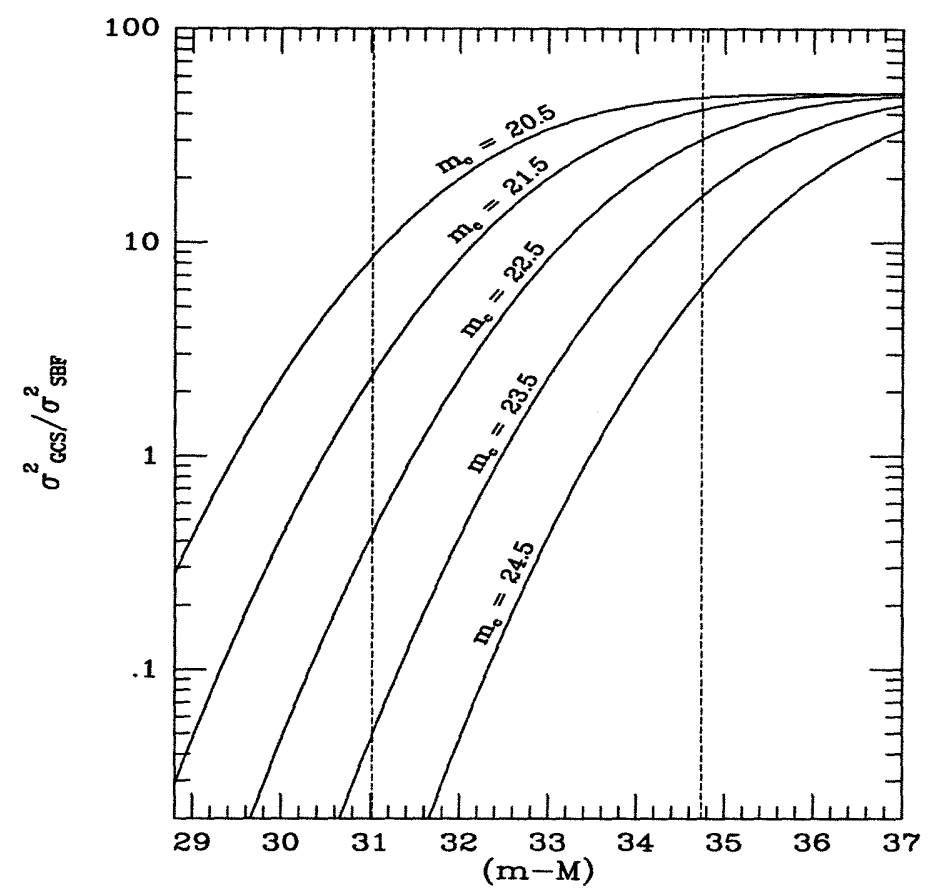

Figure 2-1: The ratio of the variances due to stellar SBF and globular clusters is plotted as a function of distance modulus for a galaxy of specific frequency $S_{N}=5$ and GCLF width $\sigma=1.4 \mathrm{mag}$. The results for five different values of the cutoff magnitude are labeled. Dashed lines represent Virgo and Coma distances. In Virgo the SBF dominate the variance for $m_{c} \gtrsim 22$, while in Coma the variance is dominated by the globular clusters for all of the represented cutoff magnitudes. 
analysis of their observations. They found that NGC 4874 was attended by a retinue of dwarf galaxies which otherwise would have been numbered among the brightest globular clusters. Pritchet \& Harris (1990) and Harris et al. (1991) further discuss the importance of excluding resolved sources. In this part of the analysis, we therefore keep only those objects classified by DoPhot either as point sources or as having insufficient signal-to-noise for shape classification to be performed, DoPhot types 1 and 7 , respectively. These objects will be referred to as "point sources."

Figure 2-2 displays the radial dependence of the number density of point sources in the magnitude ranges $20.0 \leq I_{0} \leq 23.5$ for NGC 4889 and $20.0 \leq I_{0} \leq 23.7$ for NGC 4874. As we do not have the benefit of comparison fields, we must use some other means for determining the background level. Faced with the same situation, Harris (1986) and McLaughlin et al. (1993) determined the background as the asymptotic value of the best-fit $r^{1 / 4}$ law. Our best-fit $r^{1 / 4}$ laws have been plotted in the figure, and they indicate background levels of $N_{b g}=21.6 \pm 3.0 \mathrm{arcmin}^{-2}$ and $N_{b g}=17.7 \pm 2.5$ $\operatorname{arcmin}^{-2}$, respectively. The innermost point shown for each galaxy is not complete; at the magnitude limits quoted, the data become complete at a radius of about half an arcminute. In doing the fits, we binned the data more finely and excluded the incomplete bins from the fitting process. As evidenced by the plots, this technique provides us with a reasonable value for the background contamination in a specified magnitude range.

Because we will be using the Virgo galaxies M49 and M87 as checks of our method of GCLF width determination, we show similar plots for the these galaxies in Figure 23 Here the points represent number density in the magnitude range $17.0 \leq I_{0} \leq 21.0$. The determined background level for M49 is $3.0 \pm 1.8 \mathrm{arcmin}^{-2}$. It was impossible to determine a reasonably precise background level for M87, so we have adopted the M49 value. This is analogous to the common practice of using a single comparison field for all galaxies in a sample; pure Poisson statistics would only negligibly increase the quoted fit error. In order to graphically display the adequacy of this value for M87, we have fitted an $r^{1 / 4}$ law with just two free parameters and the background fixed at $3.0 \operatorname{arcmin}^{-2}$; this fit is what we have plotted in Figure 2- 


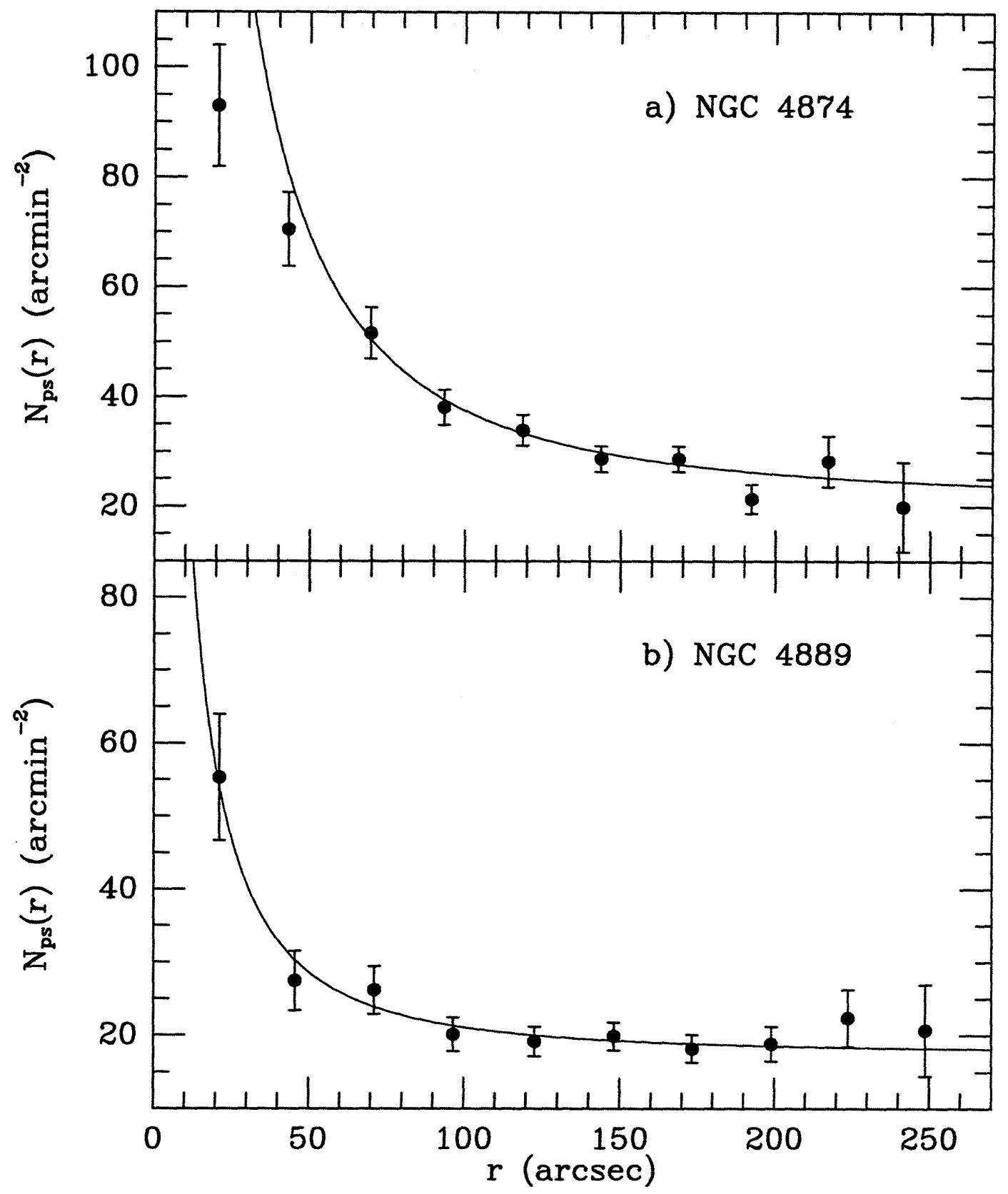

Figure 2-2: The radial dependence of the number density of point sources around the Coma galaxies in the magnitude ranges (a) $20.0 \leq I_{0} \leq 23.7$ and (b) $20.0 \leq I_{0} \leq 23.5$. The innermost point in each panel is not complete. The plotted best-fit $r^{1 / 4}$ laws are used for estimating the background levels. 


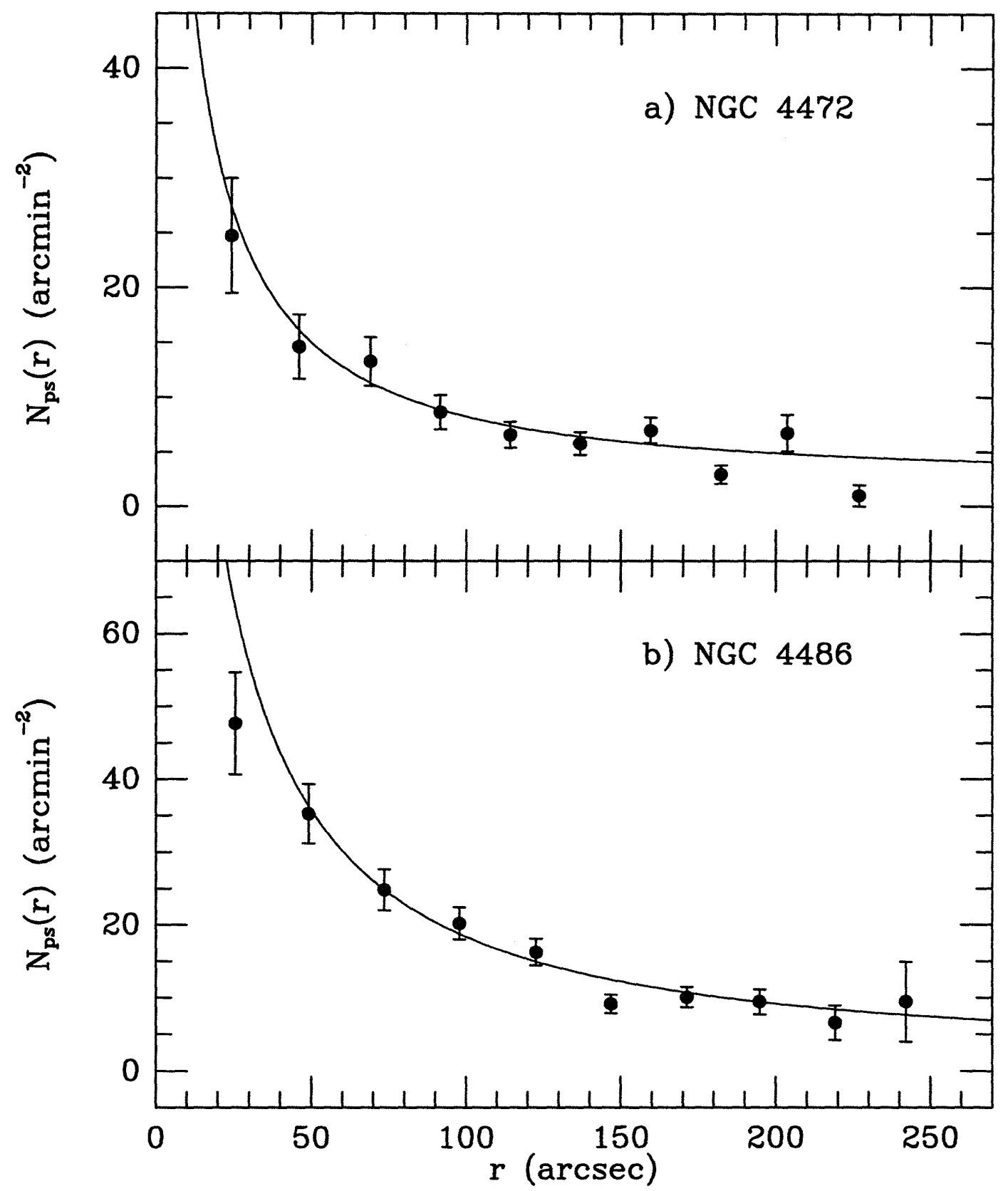

Figure 2-3: The radial dependence of the number density of point sources around the Virgo galaxies in the magnitude range $17.0 \leq I_{0} \leq 21.0$. Again, the innermost points are not complete, and the plotted best-fit $r^{1 / 4}$ laws provide estimates of the background. 
$3 b$. We can check for consistency's sake that the background values agree with the slope of the galaxy magnitude distribution from equation 2.8. Our results predict $\gamma \approx \log (21.6 / 3.0) /(23.7-21.0) \approx 0.32$, in close agreement with the values mentioned previously.

Since the completeness limit varies as a function of radius, we use several different cutoff magnitudes for each Coma galaxy, redetermining the background for each cutoff by fitting $r^{1 / 4}$ laws to the integrated counts. Figure 2-2 is representative of the fits. For the Virgo galaxies, we selected a single bright cutoff. We make no attempt to fit the slope of the GCLF's bright end with a Gaussian model, as our cutoff is several magnitudes brightward of the turnover in Coma; we simply add up the number of globulars in a given magnitude range. We combine these counts with the fluctuation measurements below to derive simultaneous constraints on the GCLF widths and the specific frequencies. Previous studies of GCS's at distances comparable to Coma have assumed a width and integrated to determine a specific frequency or estimated the specific frequencies by analogy with M87, the galaxy with largest measured GCLF width (Harris 1991). This sort of approach can lead to very large errors in the inferred specific frequencies. For instance, if the cutoff is 3 magnitudes brighter than the turnover and the GCS is assumed to be like that of M87 with $\sigma=1.7 \pm 0.2$ (Harris et al. 1991; McLaughlin et al. 1994 [a more recent HST measurement discussed in later chapters found a smaller value]) but is really more similar to that of M49 ( $\sigma=1.47 \pm 0.08$, Secker \& Harris 1993), then the calculated $S_{N}$ will be too small by a factor of 1.9 , and if it is actually closer to that of NGC $4649(\sigma=1.26 \pm 0.08$, Secker \& Harris) then the derived $S_{N}$ will be too small by a factor of 4.5 , making an extremely anomalous GCS $\left(S_{N} \approx 20\right)$ look run-of-the-mill $\left(S_{N} \lesssim 5\right)$.

Finally, we note from Figure 2-2 that the GCS of NGC 4874 is significantly more extended than that of NGC 4889, in accord with the former's status as cD. Likewise, Figure 2-3 shows that the GCS of M87 is considerably more extended than that of M49. We find no evidence for the possible deficit of clusters within $20^{\prime \prime}$ of the center of NGC 4874 reported by Harris (1987). We further discuss the extent and number of globular clusters around these galaxies, and cD galaxies in general, in Section 4. 


\subsubsection{Fluctuation Measurements}

In the SBF method, the power spectrum of a given region in the masked residual image is modeled as the sum of a component convolved with the normalized point spread function and a "white noise" component. The former component is represented by the quantity $P_{0}$, which has units of $\left(e^{-} / \mathrm{pixel}\right)^{2}$ and is equal to the total psf-convolved variance in the region. We have measured the power spectra of several annuli in each image and fitted for the $P_{0}$ values in the usual manner, detailed by Tonry et al. (1990) and Jacoby et al. (1992).

Table 2.1 provides a summary of our counts and fluctuation measurements for NGC 4874 and NGC 4889. The "region" column indicates the image and annulus in that image, where $\mathrm{c} 1$ has inner and outer radii of 32 and 64 pixels, and $\mathrm{c} 2, \mathrm{c} 3$, and $\mathrm{c} 4$ have outer radii of 128,256 , and 512 pixels, respectively; each having an inner radius equal to the next smaller one's outer radius. We also list the mean radius in arcseconds of each annulus after the mask is applied, the mean galaxy surface brightness in $\mathrm{mag} / \operatorname{arcsec}^{2}$ after masking, the chosen cutoff magnitude, the fitted $P_{0}$ in units of $10^{3}\left(e^{-} / \mathrm{pixel}\right)^{2}$, the variance $\sigma_{G C S}^{2}$ from globular clusters fainter than the cutoff, also in units of $10^{3}\left(e^{-} / \text {pixel }\right)^{2}$, the total number of point sources per $\operatorname{arcmin}^{2}$ fainter than $I=20$ but brighter than $m_{c}$, and the number per $\operatorname{arcmin}^{2}$ of globular clusters over the same magnitude range after subtracting the fitted background density. We have performed artificial star experiments to check that the data are complete to the listed cutoff magnitudes. The listed mean surface brightnesses include the light of the small companion galaxies which were modeled and subtracted along with the central galaxy, three for NGC 4874 and one for NGC 4889.

We arrived at the tabulated $\sigma_{G C S}^{2}$ values by subtracting from $P_{0}$ the estimated contributions from the stellar SBF and the background galaxies. The listed errors include the uncertainties in these quantities. Fitting for the background galaxy normalization $T_{n}$ (see equation 2.9) as one component of a two-component model which included globular cluster and background galaxy luminosity functions, we found $T_{n}=1.03$ for NGC 4874 and $T_{n}=1.02$ for NGC 4889 . Thus, we chose to take $T_{n}=1.0$, with an uncertainty of $25 \%$ as in Tonry et al. (1990). 
Table 2.1: Fluctuation Measurements and Counts for Coma Galaxies

\begin{tabular}{crccrrrrrrrr}
\hline \hline Region & \multicolumn{1}{c}{$\langle r\rangle$} & $\left\langle\mu_{I}\right\rangle$ & $m_{c}$ & \multicolumn{1}{c}{$P_{0}$} & \pm & $\sigma_{G C S}^{2}$ & \pm & $N_{p s}$ & \pm & $N_{G C}$ & \pm \\
\hline N4874.c1 & 16.6 & 20.3 & 23.3 & 698 & 34 & 614 & 38 & 37.7 & 10.9 & 27.7 & 11.1 \\
N4874.c2 & 32.8 & 21.1 & 23.5 & 311 & 8 & 252 & 15 & 52.3 & 6.6 & 36.3 & 7.1 \\
N4874.c3 & 66.9 & 22.3 & 23.7 & 143 & 6 & 101 & 11 & 52.7 & 3.6 & 31.1 & 4.7 \\
N4874.c4 & 130.5 & 23.6 & 23.7 & 89 & 6 & 50 & 11 & 31.0 & 1.4 & 9.4 & 3.3 \\
N4889.c1 & 16.2 & 19.9 & 23.2 & 394 & 30 & 318 & 33 & 35.6 & 11.3 & 26.2 & 11.5 \\
N4889.c2 & 32.6 & 21.1 & 23.4 & 192 & 11 & 144 & 15 & 29.5 & 5.2 & 14.8 & 6.2 \\
N4889.c3 & 65.3 & 22.1 & 23.5 & 126 & 5 & 87 & 10 & 25.2 & 2.5 & 7.5 & 3.5 \\
N4889.c4 & 130.1 & 24.1 & 23.5 & 62 & 6 & 26 & 10 & 18.9 & 1.1 & 1.1 & 2.7 \\
\hline \hline
\end{tabular}

\subsubsection{Constraints on Coma GCS Properties}

For an assumed width $\sigma$, we can convert the variance $\sigma_{G C S}^{2}$ to the magnitudeintegrated surface density of globular clusers $N_{0}$ by using equation 2.5 , and plugging in $m_{1}^{*}=33.54$ for NGC 4874 and $m_{1}^{*}=33.39$ for NGC 4889 . Likewise, we can convert the $N_{G C}$ values listed in Table 2.1 to total surface densities by assuming a value for $\sigma$ and integrating over the GCLF. These two determinations will be consistent over some range in $\sigma$. Figure 2-4 illustrates the situation for the c2 region of NGC 4874.

We now employ the $\chi^{2}$ formalism with two degrees of freedom to construct contours of constant $\Delta \chi^{2}$ in the $\sigma-N_{0}$ plane. The $\chi^{2}$ calculation is simply:

$$
\chi^{2}=\left(\frac{N_{0}-N_{0}^{f l u c}(\sigma)}{\delta N_{0}^{f l u c}}\right)^{2}+\left(\frac{N_{0}-N_{0}^{\text {cnt }}(\sigma)}{\delta N_{0}^{\text {cnt }}}\right)^{2}
$$

where $N_{0}^{f l u c}(\sigma)$ and $N_{0}^{\text {cnt }}(\sigma)$ are the values of $N_{0}$ determined from the fluctuations and counts, respectively, at a specific value of $\sigma$, and the denominators represent the uncertainties in these quantities. The $\chi^{2}$ contours can be equivalently represented in the $\sigma-S_{N}$ plane, as in Figures 2-5 and 2-6. (In calculating $S_{N}$, we have used an approximate $V-I$ color of 1.2 for the galaxy.) These constant $\Delta \chi^{2}$ contours are confidence contours, and if the errors are normally distributed, the level of confidence is known quantitatively from the chi-squared probability distribution (e.g. Press et al. 1992). In this discussion, we will make the assumption of normally distributed errors so that the contours can be described as representing definite levels of confidence. 


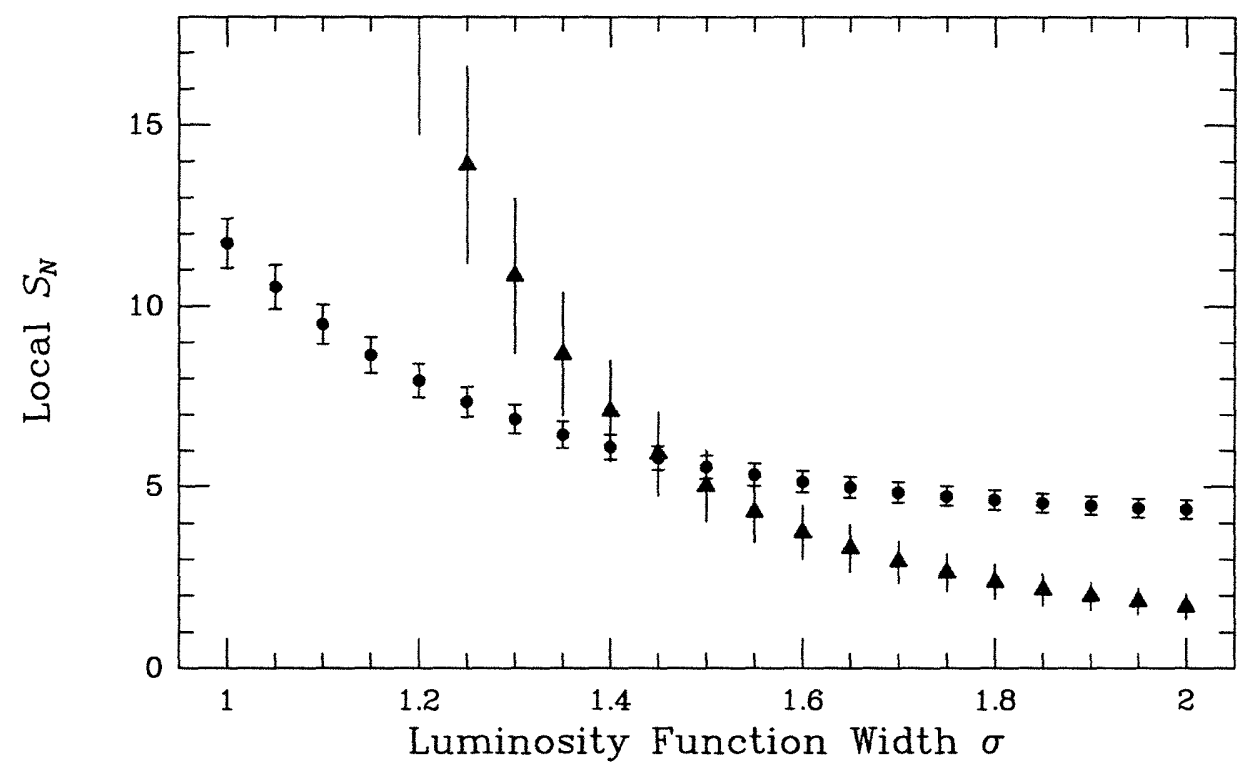

Figure 2-4: The local globular cluster specific frequency $S_{N}$ calculated for the c2 region of NGC 4874 from the results listed in Table 2.1 and for a range in GCLF width. The $S_{N}$ values derived from the bright end counts are represented by solid triangles; those derived from the fluctuation measurements are shown as filled circles. The two types of measurement yield consistent values of $S_{N}$ for $\sigma$ near 1.45 mag.

For instance, with two degrees of freedom, $\Delta \chi^{2}=2.30$ encloses the $68 \%$ confidence region, and $\Delta \chi^{2}=6.17$ encloses the $95 \%$ confidence region. These are the contours plotted in the figures.

The figures illustrate the appreciable covariance between the quantities $S_{N}$ and $\sigma$, with $S_{N}$ in Coma becoming particularly sensitive to the value of $\sigma$ for $\sigma \lesssim 1.1$. The strongest joint constraints result when the allowed values of $\sigma$ are relatively large, as is the case for the $\mathrm{c} 2$ and $\mathrm{c} 3$ regions of NGC 4874. In Table 2.2, we summarize the information contained in the figures. For each region, we first list the most probable values for $\sigma$ and $S_{N}$ with their single parameter asymmetric error bars. These single parameter uncertainties are smaller than the projections of the two-dimensional $68 \%$ confidence contours because they correspond to $\Delta \chi^{2}=1.0$, as appropriate for the chi-squared distribution with one degree of freedom. They can be viewed as the result of integrating the probabilities over the second dimension, then finding the one-dimensional, asymmetric $68 \%$ confidence intervals. They are somewhat less useful 


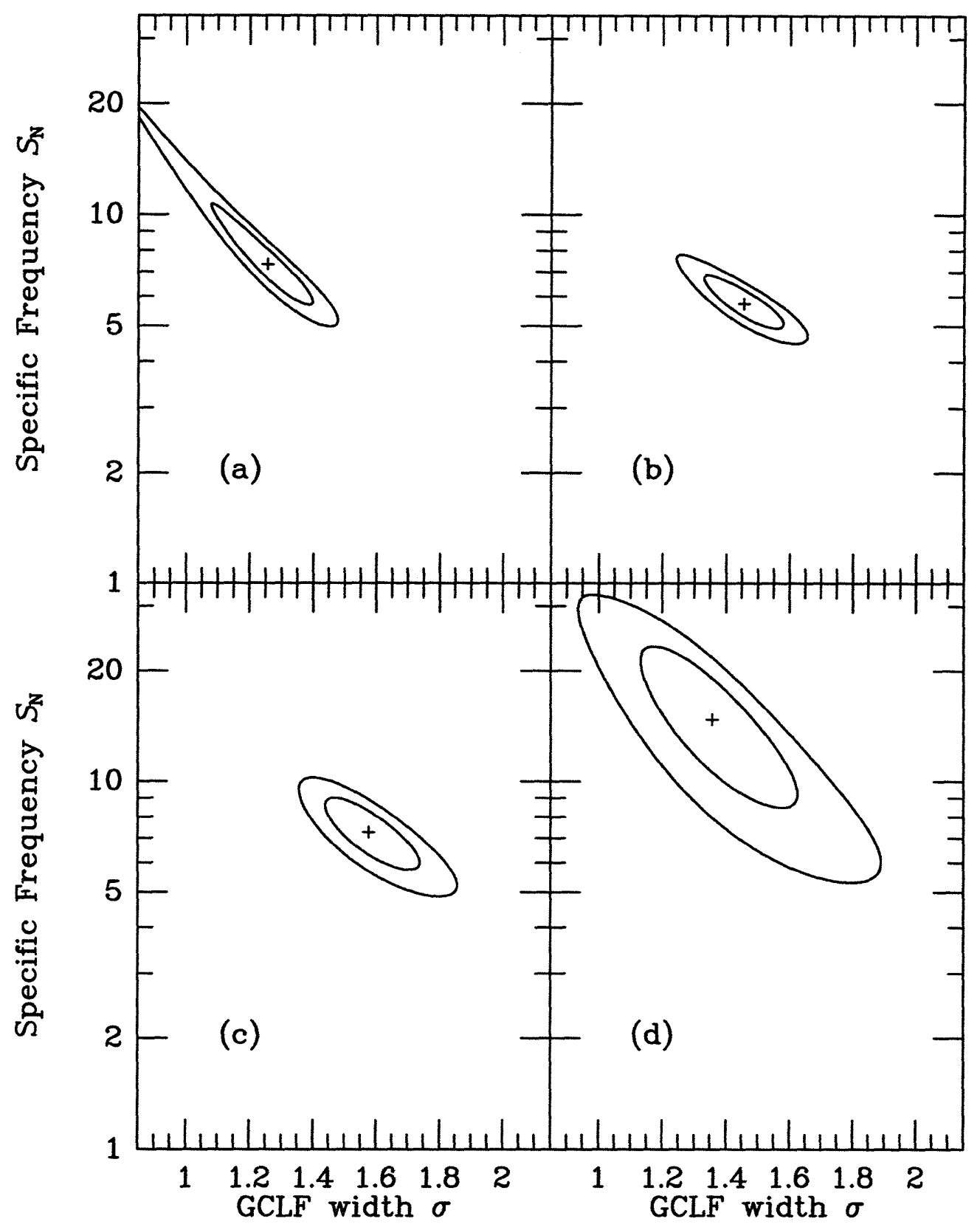

Figure 2-5: Contours of $68 \%$ and $95 \%$ joint confidence on $S_{N}$ and $\sigma$ are plotted for NGC 4874 in the regions $\mathrm{c} 1$ (a), c2 (b), c3 (c), and c4 (d), described in the text. Useful constraints are found in all four of the regions. In converting from number densities to specific frequencies, we have used our own surface photometry and an assumed $V-I$ color of 1.2 ; thus, these specific frequencies should be considered approximate. 


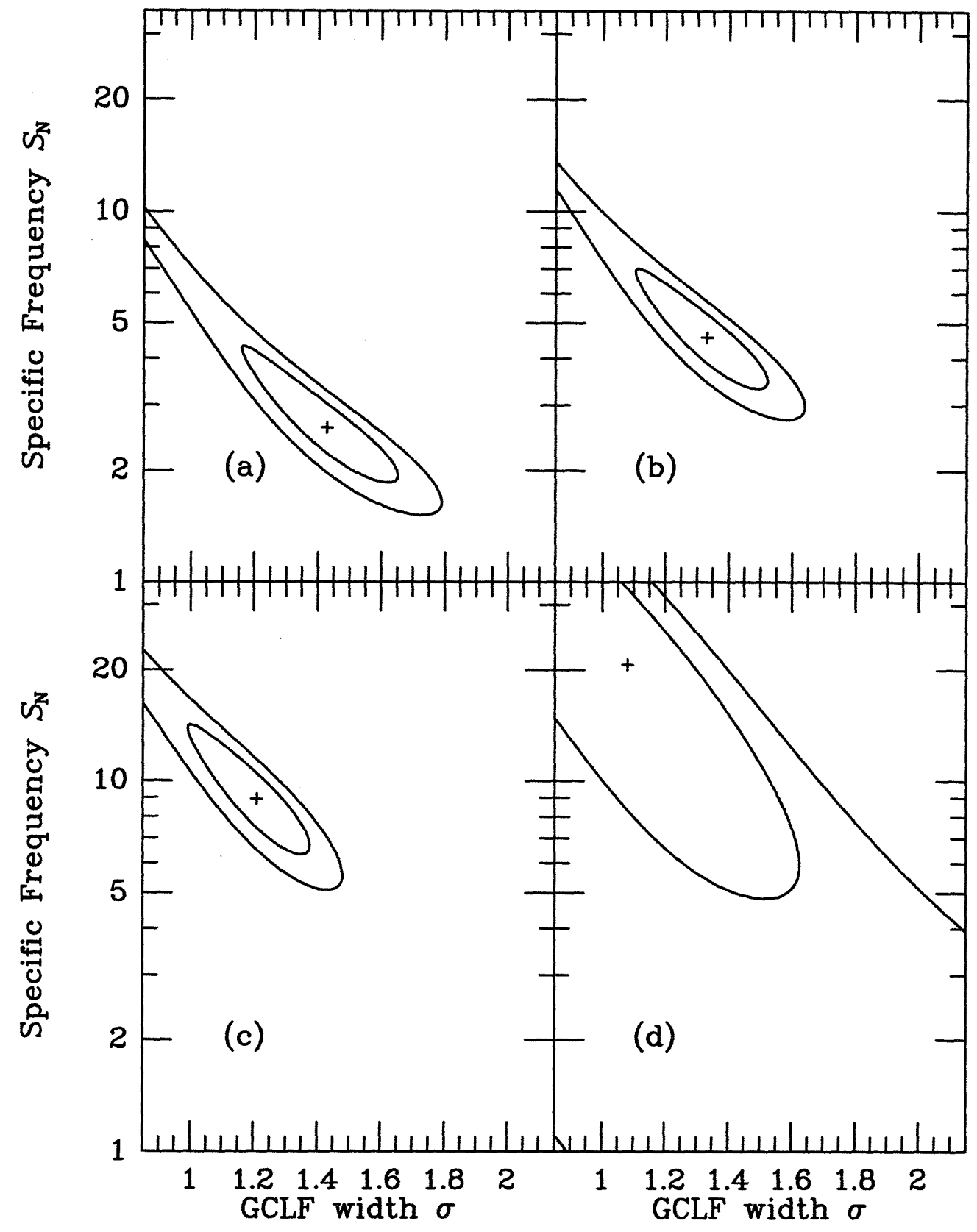

Figure 2-6: Contours of $68 \%$ and $95 \%$ joint confidence on $S_{N}$ and $\sigma$ are plotted as in Fig. 4, but this time for NGC 4889. All of the contours close except for those of the c4 region (d), where $S_{N}$ and $\sigma$ cannot be constrained. 
Table 2.2: GCS Properties for Coma Galaxies

\begin{tabular}{ccccccc}
\hline \hline Region & $\sigma(\mathrm{mag})$ & \multicolumn{1}{c}{$S_{N}$} & \multicolumn{1}{c}{$S_{N, \text { fluc }}^{\sigma_{\text {ave }}}$} & \pm & $S_{N, \text { cnt }}^{\sigma_{\text {ave }}}$ & \pm \\
\hline N4874.c1 & $1.26_{-0.11}^{+0.09}$ & $7.32_{-1.16}^{+1.82}$ & 5.7 & 0.4 & 3.2 & 1.3 \\
N4874.c2 & $1.46_{-0.08}^{+0.08}$ & $5.75_{-0.58}^{+0.68}$ & 5.9 & 0.3 & 6.3 & 1.2 \\
N4874.c3 & $1.58_{-0.09}^{+0.10}$ & $7.25_{-1.03}^{+1.13}$ & 8.0 & 0.9 & 11.0 & 1.7 \\
N4874.c4 & $1.36_{-0.15}^{+0.16}$ & $14.7_{-4.31}^{+5.27}$ & 13.9 & 3.1 & 11.6 & 4.1 \\
N4889.c1 & $1.43_{-0.17}^{+0.15}$ & $2.61_{-0.54}^{+0.88}$ & 2.8 & 0.3 & 3.4 & 1.5 \\
N4889.c2 & $1.33_{-0.14}^{+0.13}$ & $4.57_{-0.89}^{+1.32}$ & 4.4 & 0.5 & 3.9 & 1.6 \\
N4889.c3 & $1.21_{-0.13}^{+0.11}$ & $8.89_{-1.82}^{+2.77}$ & 7.2 & 0.8 & 4.1 & 2.0 \\
N4889.c4 & $1.08_{-\infty}^{+0.33}$ & $20.6_{-12.1}^{+\infty}$ & 13.2 & 5.3 & 4.0 & 9.2 \\
\hline \hline
\end{tabular}

than the two-dimensional contours because they hide the parameters' covariance.

Now, $S_{N}$ will generally depend upon position in the galaxy, but we expect $\sigma$ to be a constant, at least within a single GCS. For NGC 4874, we derive a mean Gaussian width of $\sigma=1.43 \pm 0.06 \mathrm{mag}$ from the values reported in the table. For NGC 4889, we argue below that the $\mathrm{c} 3$ and $\mathrm{c} 4$ regions may not be trustworthy, so we average the results of just the inner two regions to find $\sigma=1.37{ }_{-0.11}^{+0.10}$ mag. If we now fix $\sigma$ to be $1.43 \mathrm{mag}$ for NGC 4874 and $1.37 \mathrm{mag}$ for NGC 4889 and calculate $S_{N}$ from the fluctuations according to equation 2.12 , we acquire the quantity $S_{N, \text { fluc }}^{\sigma_{a v e}}$ listed in column 4 of Table 2.2; column 5 reports its error. Columns 6 and 7 are the results and their errors of calculating $S_{N}$ from the bright end counts with these same fixed average values of $\sigma$. A comparison of columns 5 and 7 clearly indicates that the specific frequencies inferred from the fluctuations are considerably more certain than those calculated from the counts.

For NGC $4874, S_{N}$ appears to stay constant near the center of the galaxy, then increase outward as the halo light falls off more quickly than the density of globular clusters. This result has been observed for several nearby ellipticals (see Harris 1991). It is not definitively demonstrated here for NGC 4889, however. The table shows that both the "most probable" values of $S_{N}$ and the $S_{N}$ values calculated from the fluctuations for a fixed $\sigma$ increase outward in NGC 4889, but the $S_{N}$ values calculated from the bright counts with a fixed $\sigma$ remain constant. This discrepancy is closely 
related to the fact that the "most probable" value of $\sigma$ decreases with radius in NGC 4889, since for galaxies at this distance, artificially increasing the fluctuations decreases the derived $\sigma$ while artificially increasing the bright counts increases it (see Figure 2-4). It should be noted, however, that all the derived $\sigma$ values are within the quoted uncertainties of their overall weighted mean.

Barring any physical explanation, there are three main reasons why $\sigma$ might appear to systematically decrease with radius: 1) we have overestimated the backgrounds for the bright counts in fitting the $r^{1 / 4}$ laws, 2) we have underestimated the background for the fluctuations, i.e., there is excess power which we have not properly accounted for, and 3) there is a systematic photometric error with distance from the center of the galaxy so that objects brighter than the quoted cutoff magnitudes are being erroneously left in the image, decreasing the bright counts and increasing the measured fluctuations. The last of these explanations is unlikely because, if true, it would be due to poor flat fielding, and we do not observe this effect in the NGC 4874 images which were taken the same night and flattened with the same flat fields. It is difficult to determine which of the two remaining explanations is correct (or more correct), but we think that the error is mostly due to an overestimate of the background counts. All other determinations of $S_{N}$ for both galaxies increase outward in a similar fashion, but the $S_{N}$ values determined from NGC 4889's bright counts remain constant. Moreover, the fitted background for NGC 4874 over the range $20.0 \leq I \leq 23.5$ is $16.0 \pm 2.6 \operatorname{arcmin}^{-2}$, lower than, but within error of, NGC 4889's $17.7 \mathrm{arcmin}^{-2}$. Decreasing the background from 17.7 to $15.0 \mathrm{arcmin}^{-2}$ over this magnitude range would bring NGC 4889's count results into close agreement with the fluctuation results. It is because of the probable presence of systematic biasing towards lower values of $\sigma$ in the two outer regions of NGC 4889 that we averaged just the results from the c1 and c2 regions, for which the fitted backgrounds were lower due to the brighter cutoff magnitudes and which are less affected by errors in the background due to their higher densities of globular clusters.

Global values for the specific frequencies can be found by integrating the globular cluster surface densities and mean galaxy surface brightnesses over the total area of 
the regions. We use the surface densities which result from the fluctuation measurements of Table 2.1 and our most likely Gaussian widths of 1.43 mag for NGC 4874 and $1.37 \mathrm{mag}$ for NGC 4889 . In order to make the resulting $S_{N}$ values as global as possible, we performed the fluctuation analysis on an annulus in each image which we call "c0", extending from 16 to 32 pixels. With $m_{c}=23.0$, we measured $P_{0}$ values of $(7.8 \pm 1.8) \times 10^{5}$ and $(10.2 \pm 0.5) \times 10^{5}\left(e^{-} / \text {pixel }\right)^{2}$ and mean galaxy surface brightnesses of 19.1 and $18.8 \mathrm{mag} / \mathrm{arcsec}^{2}$ for NGC 4874 and NGC 4889 , respectively. The smallness of the annulus precluded any further constraints on the GCLF widths. Over the entire area of study in each image, we calculate the total number of globular clusters to be $13060 \pm 1540$ for NGC 4874 and $10150 \pm 1780$ for NGC 4889 . Correcting for the masked pixels in our images within a radius of 512 pixels and for the area within 16 pixels which was not studied, we find total globular cluster populations of $N_{t}=17260 \pm 2030$ for NGC 4874 and $N_{t}=13200 \pm 2320$ for NGC 4889. At our adopted distance modulus, a radius of 512 pixels projects to $75 \mathrm{kpc}$, which will contain the vast majority of the globular clusters, so we make no correction for those which may lie outside this region. We also make no correction for globular clusters within this region which might belong to the companion galaxies that were modeled and subtracted from the image. These two corrections would be small, uncertain, and work in opposite directions. Because of the uncertain accuracy of our surface photometry and the lack of measured $V-I$ colors for these galaxies, we adopt the total apparent $V$ magnitudes from de Vaucouleurs et al. 1976: $V(\mathrm{~N} 4874)=12.0$ and $V(\mathrm{~N} 4889)=11.5$. Finally, we arrive at global specific frequencies of $S_{N}=14.3 \pm 1.7$ for NGC 4874 and $S_{N}=6.9 \pm 1.2$ for NGC 4889 , yielding the ratio $S_{N}^{N 4874} / S_{N}^{N 4889}=2.1 \pm 0.4$. Uncertainties in the GCLF width and mean add an additional $\sim 20 \%$ uncertainty, implying $S_{N}=14.3 \pm 3.3$ and $S_{N}=6.9 \pm 1.8$, respectively. $\left[S_{N}\right.$ for is revised downward for NGC 4874 in Chapter 5 due to better photometry.]

Harris (1987) assumed identical Gaussian widths for these galaxies and calculated the ratio of their specific frequencies to be $2.8 \pm 0.7$, in good agreement with our results, especially since the assumption of $\sigma=1.43 \mathrm{mag}$ for NGC 4889 as well as NGC 4874 would decrease the former's $S_{N}$ to 6.5 . Referencing Harris's study, McLaughlin, 
Table 2.3: Fluctuation Measurements and Counts for Virgo Galaxies

\begin{tabular}{crrrrrrrrrrr}
\hline \hline Region & \multicolumn{1}{c}{$\langle\boldsymbol{r}\rangle$} & $\left\langle\mu_{I}\right\rangle$ & $m_{c}$ & \multicolumn{1}{c}{$P_{0}$} & \pm & $\sigma_{G C S}^{2}$ & \multicolumn{1}{c}{ \pm} & $N_{p s}$ & \pm & $N_{G C}$ & \pm \\
\hline N4472.c1 & 16.5 & 17.5 & 21.0 & 2253 & 123 & 565 & 203 & 26.8 & 9.8 & 23.8 & 9.6 \\
N4472.c2 & 32.8 & 18.5 & 21.0 & 1324 & 55 & 620 & 85 & 17.0 & 3.7 & 14.0 & 4.1 \\
N4472.c3 & 66.7 & 19.7 & 21.0 & 704 & 14 & 403 & 34 & 13.8 & 1.7 & 10.8 & 2.5 \\
N4472.c4 & 129.4 & 21.0 & 21.0 & 364 & 8 & 209 & 25 & 6.3 & 0.6 & 3.3 & 1.9 \\
N4486.c1 & 16.7 & 17.6 & 21.0 & 3039 & 91 & 1664 & 159 & 52.6 & 13.2 & 49.6 & 13.3 \\
N4486.c2 & 33.3 & 18.7 & 21.0 & 2139 & 41 & 1574 & 66 & 43.9 & 6.0 & 40.9 & 6.2 \\
N4486.c3 & 66.3 & 19.9 & 21.0 & 1068 & 13 & 827 & 29 & 26.6 & 2.3 & 23.6 & 2.9 \\
N4486.c4 & 130.1 & 21.4 & 21.0 & 492 & 10 & 368 & 24 & 13.6 & 0.9 & 10.6 & 2.0 \\
\hline \hline
\end{tabular}

Harris, \& Hanes (1994) report $12 \pm 6$ as the specific frequency in NGC 4874 . We have confirmed that NGC 4874 suffers from the high- $S_{N}$ anomaly, while NGC 4889 has a specific frequency typical of large elliptical galaxies.

\subsubsection{Results for Virgo Galaxies}

It is gratifying that our fluctuation analysis has produced results for the specific frequencies in Coma which agree so closely with Harris's direct counting results; however, we would like to know if our measured GCLF widths are similarly accurate. Thus, we apply the same analysis to the Virgo galaxies M49 and M87, which are close enough to have their GCLF widths measured via direct counting. In Table 2.3 we list the results of our fluctuation and counting analyses. The columns are the same as those of Table 2.1. The $m_{1}^{*}$ values are 32.48 for the M49 image and 32.44 for the M87 image.

Although the observed number densities of globular clusters brighter than the cutoff magnitudes are comparable to those for the Coma galaxies, the relative contributions from the globular clusters to the $P_{0}$ values are much less. The reason for this is two-fold: first, the total surface density of globular clusters will increase as the square of the distance, and second, our cutoff, though about 2.5 mag brighter than the cutoff in Coma, lies approximately a magnitude fainter along the GCLF. We chose a relatively bright cutoff in order to avoid depleting the fluctuation power from globular clusters. For instance, in the $\mathrm{c} 3$ region of M49, globular clusters contribute 
$57 \%$ of the measured $P_{0}$ for $m_{c}=21.0$, as shown in the table. Pushing the cutoff to $m_{c}=22.0$, at which the data in this region are still complete, we find that only $22 \%$ of the variance is due to globular clusters, making the conversion to a number density much less certain and thereby weakening the constraints on $\sigma$. Our choice of $m_{c}$ strikes a happy medium between plentiful direct counts and a power spectrum dominated by globular clusters.

Since we wish to test our method of GCLF width determination, we have used the same GCLF turnover values as were found by those studies which reported the widths. Employing the same assumptions as before, we therefore take $m_{I}^{0}=22.86$ for M49 (Secker \& Harris 1993) and $m_{I}^{0}=23.18$ for M87 (McLaughlin et al. 1994). Figures 2-7 and 2-8 display the resulting joint confidence contours on $\sigma$ and $S_{N}$ for these galaxies. For M49, the most probable values of $\sigma$ and their one-dimensional error bars are $2.01{ }_{-0.50}^{+0.75}, 1.49_{-0.20}^{+0.21}, 1.61 \pm 0.18$, and $1.29_{-0.31}^{+0.27} \mathrm{mag}$, for the $\mathrm{c} 1, \mathrm{c} 2$, $\mathrm{c} 3$, and $\mathrm{c} 4$ regions, respectively. The $\mathrm{c} 1$ region produces only a very weak constraint because its power spectrum is dominated by the stellar SBF. Averaging the three regions with nearly symmetric error bars, we find $\sigma=1.51 \pm 0.13 \mathrm{mag}$, in excellent agreement with the value of $1.47 \pm 0.08$ mag from Secker \& Harris. Moreover, the results from the individual regions all agree with this value.

For the abundant GCS of M87, we determine most probable values of $\sigma$ in the c1-c4 regions of $1.75 \pm 0.22,1.65 \pm 0.11,1.72 \pm 0.09$, and $1.72 \pm 0.15 \mathrm{mag}$, which are so internally consistent as to suggest that we may be overestimating the errors in this particular case. Taking an average, we find $\sigma=1.70 \pm 0.07$, in remarkable agreement with the best fit value of 1.73 mag from McLaughlin et al. (1994). Thus, we confirm the reliability of this method for measuring $\sigma$, given $m^{0}$. We stress that these results do not necessarily confirm the abnormally large width in M87, however. Had we assumed a turnover which was brighter by $0.4 \mathrm{mag}$, our derived width would have been smaller by $\sim 0.2 \mathrm{mag}$, making it very similar to the other Virgo GCLFs. In fact, McLaughlin et al. also obtain a formally acceptable fit with $\sigma=1.50$ and $m_{V}^{0}=23.65\left(m_{I}^{0}=22.65\right)$. More optimistically, we can expand on their finding that the GCLF width is constant outside a radius of $1^{\prime} .21$; our results indicate that it 


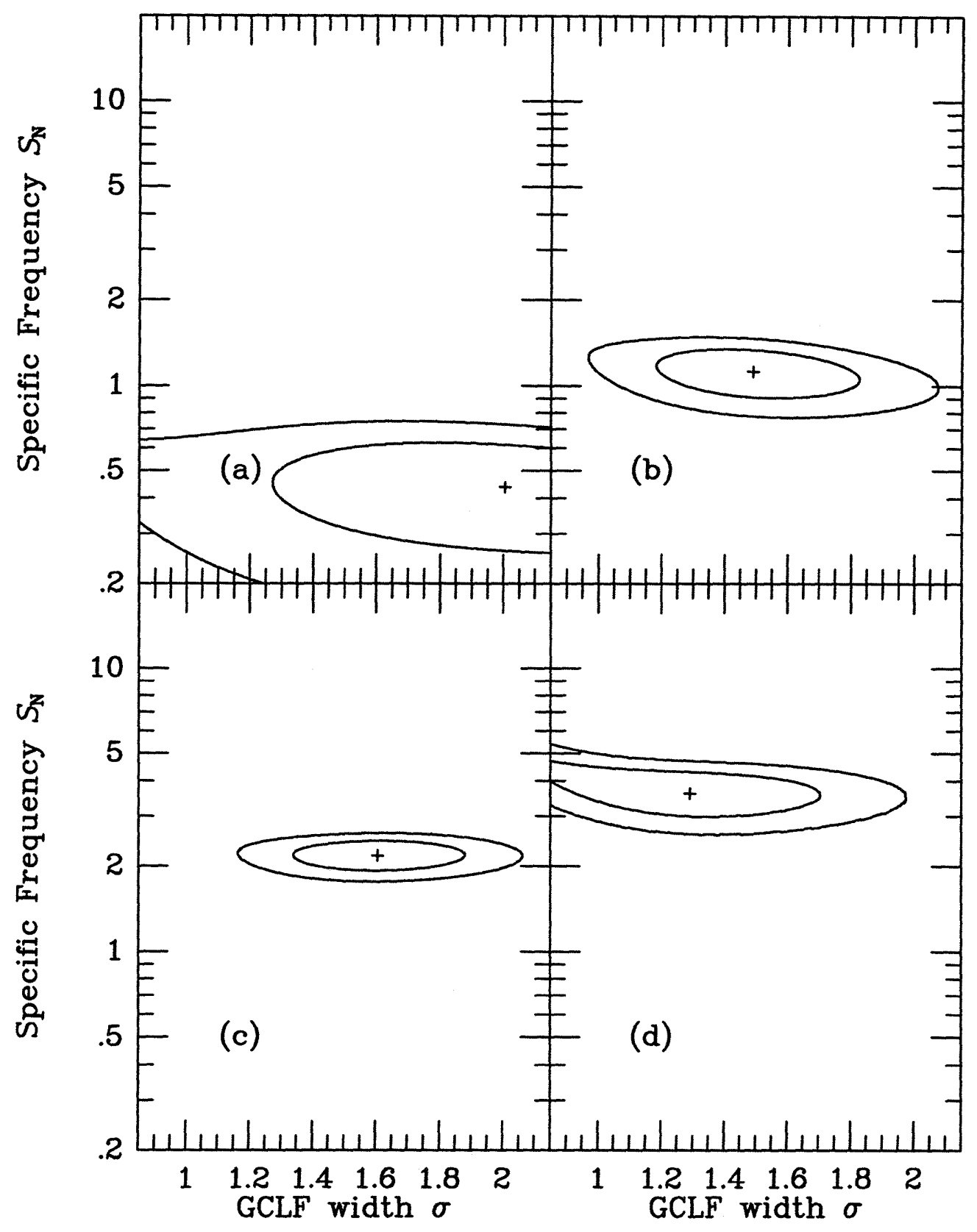

Figure 2-7: Contours of $68 \%$ and $95 \%$ joint confidence on $S_{N}$ and $\sigma$ as in Fig. 4, but here for the Virgo galaxy NGC 4472 (M49). All of the contours close, though only very weak constraints can be placed for the $\mathrm{c} 1$ region (a). 


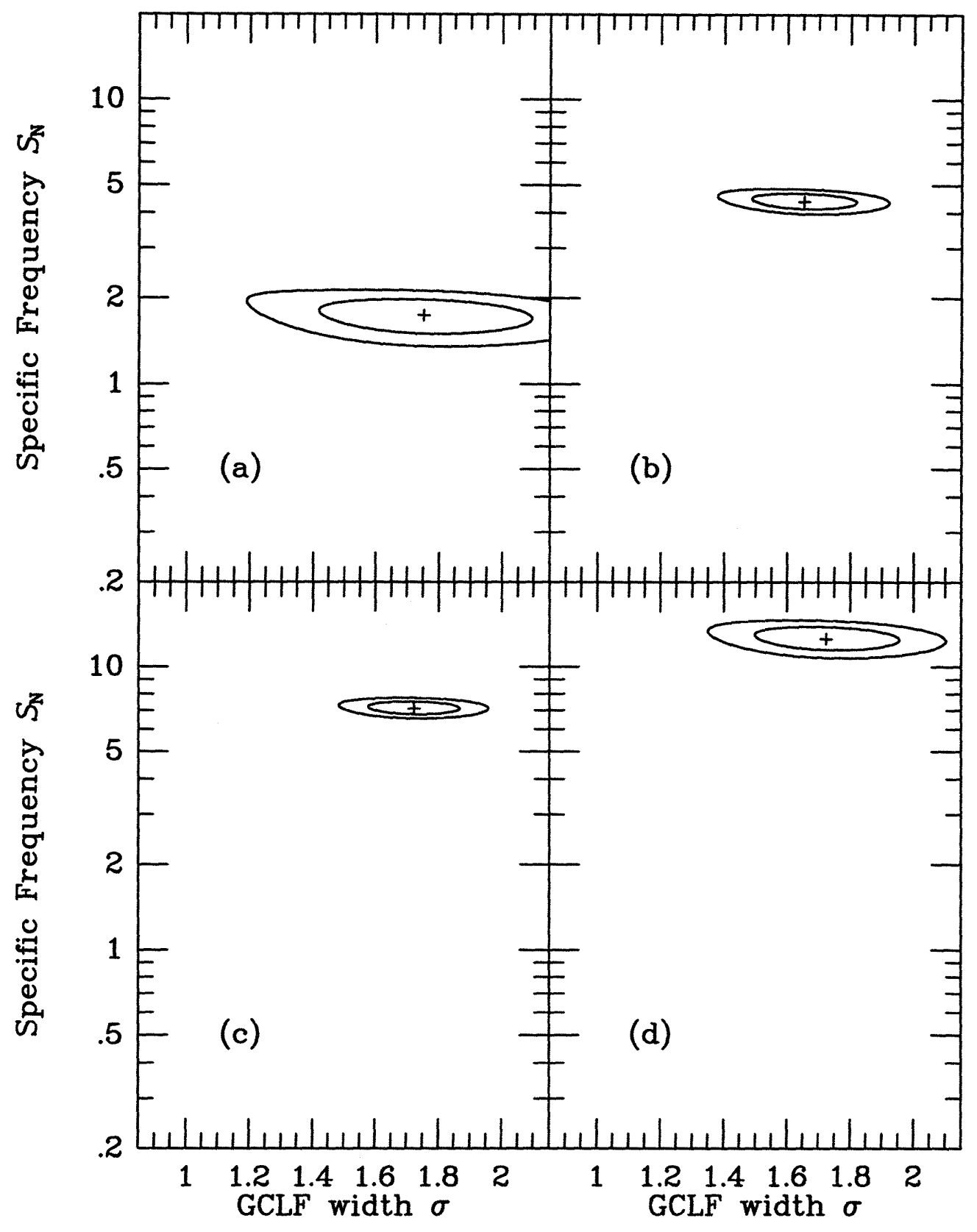

Figure 2-8: Contours of $68 \%$ and $95 \%$ joint confidence on $S_{N}$ and $\sigma$ as in Fig. 4, but here for the Virgo galaxy NGC 4486 (M87). The values are nicely constrained in each of the four regions. 
remains nearly constant inward to at least $\sim 0^{\prime} .25$. Finally, we note that the most likely $S_{N}$ for each region in M87 is a factor of 3-4 larger than the corresponding value in $\mathrm{M} 49$, in accord with previous findings, as are the individual values themselves at these radii (cf. Fig. 21 of McLaughlin et al. [1994]).

\subsection{Discussion}

\subsubsection{The GCLF Parameters}

The GCLF method of distance determination for nearby galaxies relies on the universality of the turnover magnitude $m^{0}$. It has been applied only to relatively nearby galaxies because of the necessity to fit for both $m^{0}$ and the width $\sigma$. Our technique provides a means for studying the variation of $\sigma$ among large ellipticals which are too distant to have their GCLF parameters fitted by direct counting. In order to learn the most from such a study, the galaxies should be members of the same cluster so that they would presumably have the same $m^{0}$. We have found that the two giant ellipticals at center of the Coma cluster have identical values of $\sigma$ to within the uncertainties. If $\sigma$ can be shown to vary by only a small amount, or in a predictable way, then it would no longer need to be fitted for, and the GCLF method could be applied to more distant galaxies.

In determining $\sigma$ for NGC 4874 and NGC 4889, we have assumed a turnover magnitude in the $I$-band of 26.50 and estimated its uncertainty at $\lesssim 0.25$. In order to incorporate this uncertainty, we changed $m^{0}$ by \pm 0.25 mag and repeated the analysis for the Coma galaxies. The resulting values of $\sigma$ changed by $\pm 0.07 \mathrm{mag}$, in the sense of larger $\sigma$ for larger $m^{0}$ and vice versa. Including this uncertainty, our final results for the widths are $\sigma=1.43 \pm 0.09 \mathrm{mag}$ for NGC 4874 and $\sigma=1.37_{-0.13}^{0.12}$

mag for NGC 4889. The average of these two, $\langle\sigma\rangle_{C o m}=1.42 \pm 0.11$ mag (adding the 0.07 mag error after averaging the two) is very close to the average $\langle\sigma\rangle_{V i r}=$ $1.39 \pm 0.12$ found by Secker \& Harris (1993) for the three large Virgo ellipticals NGC 4365, NGC 4472, and NGC 4649. Thus, it appears that large ellipticals do indeed have Gaussian widths very near 1.4 mag. One obvious outlier is M87, whose width 
appears to be larger by $\sim 0.3 \mathrm{mag}$ [HST measurements discussed later in the thesis find that M87's width had indeed been overestimated.] This is not a common feature of high- $S_{N}$ galaxies, as NGC 1399 (Geisler \& Forte 1990; Bridges, Hanes, \& Harris 1991) and NGC 4874 both appear to have normal widths. In order to firmly establish whether or not M87 has a significantly different luminosity function, deeper imaging of this extremely important GCS is necessary.

For this study, we chose to adopt the Gaussian formalism in discussing the GCLF because it is currently the most useful one for purposes of comparison. Many more galaxies have measured Gaussian widths and means than any other type of luminosity function parameter. However, the " $t_{5}$ " distribution of Secker (1992) appears to fit the Virgo GCLFs slightly better (Secker \& Harris 1993). Our method for measuring the luminosity function width depends only upon the existence of a known, accurate functional form for the GCLF; it can be applied in the future using another formalism, such as that of the $t_{5}$ distribution.

\subsubsection{Specific Frequencies and cD Galaxies}

Our results strongly bolster one of the $\sim 10$ data points of McLaughlin et al. (1994) which imply that high- $S_{N} \mathrm{cD}$ 's predominate in less dynamically evolved clusters, as characterized somewhat subjectively by the Bautz-Morgan (BM) types. Our measurement of $S_{N}$ for NGC 4874 is $3 \sigma$ above the norm for large ellipticals, as are the $S_{N}$ values of M87 and NGC 1399. These and the other apparently high- $S_{N}$ central galaxies, apart from UGC 9958, all reside in clusters of BM type II or III, while the central galaxies in BM type I clusters possess normal specific frequencies. Since BM I clusters are believed to be the most dynamically evolved, McLaughlin et al. suggested that the central galaxies in these clusters have had time to dilute their populations of globular clusters through mergers with the normal $S_{N}$ cluster galaxies. Evidence from multiple nucleus velocities in brightest cluster galaxies appears to support this $S_{N^{-}}$ dilution hypothesis. Blakeslee \& Tonry (1992) reported that low-velocity secondary nuclei, indicative of imminent merging, occur preferentially in clusters of BM types II-III and III and suggested that central galaxies in more evolved clusters had already 
consumed their low-velocity neighbors.

How these high- $S_{N}$ galaxies initially acquired their "extra" portion of globular clusters remains in debate. McLaughlin et al. (1994) contend on structural grounds that the excess globular clusters of M87 must have been added along with the cD envelope in a gaseous state, very early in the cluster history and not during its subsequent evolution. While this may seem a natural argument to go with the hypothesis that high- $S_{N}$ systems become diluted through mergers, it is not the only possibility. For instance, they remark in a footnote that tidal stripping of the high- $S_{N}$ outskirts (recall $S_{N}$ increases with radius) of other Virgo galaxies could increase M87's specific frequency, but they reject this scenario as the principal means of producing high- $S_{N}$ systems because of the improbability that enough globulars could be added. For this mechanism to work, it would have to take place on a timescale much shorter than the merging timescale so that the specific frequency could increase substantially due to stripping before becoming diluted through full-blown mergers. In Virgo, there are at least 12 galaxies besides M87 with 1000 or more globular clusters (Harris 1991), the total population of which exceeds 40000 . In order to account for M87's excess, these galaxies would have had to have lost the outer $20 \%$ of their original GCSs, contributing part of the $\mathrm{cD}$ envelope in the process. Merritt (1988) finds that galaxies which pass near a central $c D$ become significantly tidally truncated after just a few orbits, contributing to the luminosity of the central galaxy. His results imply that a galaxy with a GCS falling off as $r^{-2}$ to a distance of $50 \mathrm{kpc}$ would lose $\sim 20 \%$ of its globular clusters if its pericenter lay at $100 \mathrm{kpc}$. Detailed numerical simulations would appear necessary to determine whether or not tidal stripping in cluster cores can in fact result in high- $S_{N}$ systems.

We have shown that the SBF technique provides an extremely promising means for measuring specific frequencies in distant galaxies. It may prove to be the best way for learning about the statistics of high- $S_{N}$ systems, including their dependence on environment, which should shed further light on their formation mechanism. If there proves to be little variation in GCLF widths among large ellipticals, the images would not need to be deep enough to show the brightest globular clusters for the 
purpose of simultaneously constraining $\sigma$, though the results will be more certain if the brightest ones are visible. Observations have already been performed for the purpose of measuring specific frequencies of several distant cluster galaxies using globular cluster fluctuations, so the future of this method appears secure.

\subsection{Summary and Conclusions}

We have developed a technique for simultaneously measuring the specific frequencies and luminosity function widths of relatively distant GCSs by using both direct counts of the brightest globular clusters and the variance in the image due to those below the limit of detection. Applying this technique to the central giants in Coma, we find $\sigma=1.43 \pm 0.09 \mathrm{mag}, S_{N}=14.3 \pm 3.3$ for NGC 4874 and $\sigma=1.37_{-0.13}^{+0.12} \mathrm{mag}$, $S_{N}=6.9 \pm 1.8$ for NGC 4889. The quoted errors include the uncertainty in the GCLF turnover $m^{0}$. The outwardly rising $S_{N}$ in these galaxies indicate that the GCSs are more extended than the halo light, as observed in several nearby ellipticals.

In order to test accuracy of this technique for measuring GCLF widths, we have applied it to the Virgo galaxies M49 and M87. Using the turnover magnitudes reported by previous studies which had fitted directly for $m^{0}$ and $\sigma$, we recovered GCLF widths in excellent agreement with the fitted values. These results give us confidence that the values of $\sigma$ determined for the Coma galaxies are reliable.

We have confirmed that the cD NGC 4874 has the high- $S_{N}$ anomaly characteristic of several other central dominant galaxies. Our results indicate that the study of fluctuations produced by globular clusters will soon reveal hitherto hidden information about the specific frequencies of other distant cluster galaxies, perhaps proving that high- $S_{N}$ central galaxies are found exclusively in clusters in earlier stages of dynamical evolution. For galaxies which are nearby enough and in possession of sufficient numbers of globular clusters, we can apply the technique developed in this paper for measuring GCLF widths as well as specific frequencies. Both types of information may eventually prove valuable in understanding how high- $S_{N}$ systems, and GCSs in general, were formed. 


\subsection{References}

Aaronson, M., Bothun, G., Mould, J., Huchra, J., Schommer, R. A., \& Cornell, M. E. 1986, ApJ, 302, 536

Ajhar, E. A., Blakeslee, J. P., \& Tonry, J. L. 1994, AJ, 108, 2087

Blakeslee, J. P. \& Tonry, J. L. 1992, AJ, 103, 1457

Bridges, T. H., Hanes, D. A., \& Harris, W. E. 1991, AJ, 101, 469

Burstein, D. \& Heiles, C. 1984, ApJS, 54, 33

Couture, J., Harris, W. E., \& Allwright, J. W. B. 1990, ApJS, 73, 671

Couture, J., Harris, W. E., \& Allwright, J. W. B. 1991, ApJ, 372, 97

de Vaucouleurs, G., de Vaucouleurs, M., \& Corwin, H. G. 1976, Second Reference Catalog of Bright Galaxies (Austin: University of Texas Press)

Faber, S. M., Wegner, G., Burstein, D., Davies, R. L., Dressler, A., Lynden-Bell, D., \& Terlevich, R. J. 1989, ApJS, 69, 763

Geisler, D. \& Forte, J. C. 1990, ApJ, 350, L5

Harris, W. E. 1986, AJ, 91, 822

Harris, W. E. 1987, ApJ, 315, L29

Harris, W. E. 1991, ARA\&A, 29, 543

Harris, W. E., Allwright, J. W. B., Pritchet, C. J., \& van den Bergh, S. 1991, ApJS, 76,115

Jacoby, G. H., Branch, D., Ciardullo, R., Davies, R. L., Harris, W. E., Pierce, M. J., Pritchet, C. J., Tonry, J. L., \& Welch, D. L. 1992, PASP, 104, 599

Landolt, A. U. 1983, AJ, 88, 439

Landolt, A. U. 1992, AJ, 104, 340

Lilly, S. J., Cowie, L. L., \& Gardner, J. P. 1991, ApJ, 369, 79

Mateo, M. \& Schechter, P. L. 1989, in First ESO/ST-ECF Data Analysis Workshop, ed. P. J. Grosb $\varnothing$ l, F. Murtagh, and R. H. Warmels (Garching: ESO), 69

McLaughlin, D. E., Harris, W. E., \& Hanes, D. A. 1993, ApJ, 409, L45

McLaughlin, D. E., Harris, W. E., \& Hanes, D. A. 1994, ApJ, 422, 486

Merritt, D. 1988, in The Minnesota Lectures on Clusters of Galaxies and Large-Scale 
Structure, ed. J. M. Dickey (ASP Conf. Ser., 5), 175

Metzger, M. R., Tonry, J. L., \& Luppino, G. A. 1993, in Astronomical Data Analysis Software and Systems II, ed R. J. Hanisch, R. J. V. Brissenden, and J. Barnes (ASP Conf. Ser., 52), 300

Press, W. H., Teukolsky, S. A., Vetterling, W. T., \& Flannery, B. P. 1992, Numerical Recipes in C, Second Edition (New York: Cambridge Univ. Press)

Pritchet, C. J. \& Harris, W. E. 1990, ApJ, 355, 410

Schechter, P. L., Mateo, M., \& Saha A. 1993, PASP, 105, 1342

Secker, J. 1992, AJ, 104, 1472

Secker, J. \& Harris, W. E. 1993, AJ, 105, 1358

Thompson, L. A. \& Valdes, F. 1987, ApJ, 315, L35

Tonry, J. L. 1991, ApJ, 373, L1

Tonry, J. L., Blakeslee, J. P., Ajhar, E. A., \& Dressler, A. 1996, ApJ, in press

Tonry, J. L., Ajhar, E. A., \& Luppino, G. A. 1990, AJ, 100, 1416

Tonry, J. L. \& Schneider, D. P. 1988, AJ, 96, 807

Tyson, J. A. 1988, AJ, 96, 1

Worthey, G. 1993, ApJ, 409, 530

Wing, D. L., Harris, G. L. H., Hanes, D. A., \& Harris, W. E. 1995, AJ, 109, 121 


\section{Chapter 3}

\section{The GCLF Next Door: Fornax and Virgo}

A shorter version of this chapter appeared as "Globular Clusters in Fornax: Does $M^{0}$ Depend on Environment?", The Astrophysical Journal, Vol. 465, L19.

\section{Synopsis}

We present the $V$-band globular cluster luminosity functions (GCLFs) of the Fornax Cluster galaxies NGC 1344, NGC 1380, NGC 1399, and NGC 1404. Our observations reach to $V=24.8$, roughly one magnitude beyond the GCLF turnover $m_{V}^{0}$, with $\sim 90 \%$ completeness. We also estimate the number of GCs fainter than this magnitude from the fluctuations they produce in the images. The GCLFs of these galaxies are well fitted by Gaussians; the weighted means of their turnover magnitudes and dispersions are $\left\langle m_{V}^{0}\right\rangle=23.88 \pm 0.10 \mathrm{mag}$ and $\langle\sigma\rangle=1.35 \pm 0.07 \mathrm{mag}$. The assumption of a universal value for the absolute magnitude of the turnover $M_{V}^{0}$ places the Fornax cluster $0.13 \pm 0.11$ mag more distant than Virgo. However, in light of recent Cepheid and other high-precision distance measurements, as well as ongoing HST observations of GCLFs for the purpose of determining the extra-galactic distance scale, we choose to re-examine the universal GCLF hypothesis. Based on data from groups and clusters of galaxies, we find evidence that $M_{V}^{0}$ becomes fainter as the local density of galaxies increases. We speculate on the possible cause of this trend; if it is confirmed, GCLF observations will be less useful for determining distances, but may provide important information for constraining theories of star formation in primordial galaxy halos. 


\subsection{Background}

Observations indicate that the globular cluster (GC) populations surrounding large galaxies follow luminosity functions of the same general log-normal form. The globular cluster luminosity function (GCLF) has often been employed as a standard-candle distance indicator based on the assumption a universal value for its mean, or turnover, magnitude $M^{0}$ (see Jacoby et al. 1992 for a review of the method). Until recently, it was impossible to apply the GCLF method to determine the distances of galaxies further away than Virgo. Now, with HST and improvements in ground-based seeing and instrumentation, it becomes potentially much more powerful for determining the extra-galactic distance scale. Furthermore, new Cepheid and other high-precision local distance measures would allow for a firm calibration of the method.

Baum et al. $(1995 \mathrm{a}, \mathrm{b})$ have used HST to observe the globular clusters (GCs) of the Coma galaxies NGC 4881 and IC 4051 down to $V=27.6$ and $V=28.4$, respectively. They derive values of the Hubble constant $H_{0}$ near $60 \mathrm{~km} / \mathrm{s} / \mathrm{Mpc}$. Also with HST, Whitmore et al. (1995) studied the GCs of the extremely rich M87 system to two magnitudes beyond $M_{V}^{0}$ (the $V$-band GCLF turnover) and derived $H_{0}=78 \pm 11$ $\mathrm{km} / \mathrm{s} / \mathrm{Mpc}$. Only a small part of the discrepancy in derived $H_{0}$ values can be accounted for by the different calibrations used by the two groups. This situation leads one to suspect that the GCLFs themselves may be intrinsically different, especially as there remains no firmly established physical basis for assuming a universal $M^{0}$. Previously, there have been suspicions that $M^{0}$ is different for spirals and ellipticals (Secker \& Harris 1993), with the root cause of this difference being metallicity variations (Ashman, Conti, \& Zepf 1995), but $M_{V}^{0}$ was assumed not to vary among large ellipticals. As Whitmore et al. candidly remark, this "crucial assumption" of a universal GCLF is "a hypothesis that needs further verification."

In this Letter, we examine the current state of the universal GCLF hypothesis. First, we present new observations of GCs around four Fornax galaxies. Fornax is an important cluster for testing distance determination methods, as it is spatially much more concentrated than Virgo while being at nearly the same distance (e.g. Tonry 
1991; Ciardullo, Jacoby, \& Tonry 1993). We find that the GCLF exhibits remarkably little variation for these Fornax galaxies. Next, we use independent distance measurements to galaxies and galaxy groups to compare derived $M_{V}^{0}$ values in different environments. We find evidence that $M_{V}^{0}$ becomes fainter as the local density of galaxies increases. Further verification is once again needed, but if the observed trend proves real, it would have major implications for the GCLF method of distance measurement as well as for theories of GC formation. We conclude with a discussion of these implications, in particular how the local galaxy environment may govern the properties of GC populations.

\subsection{Observations and Reductions}

We observed the Fornax Cluster galaxies NGC 1316, NGC 1344, NGC 1380, NGC 1399, and NGC 1404 in 1995 August with the Tek 2048 \#4 CCD detector at the Cassegrain focus of the $4 \mathrm{~m}$ telescope at Cerro Tololo. Four $600 \mathrm{~s} V$-band exposures were taken of each galaxy, except NGC 1316, for which five $600 \mathrm{~s}$ exposures were taken. We also obtained $2400 \mathrm{~s}$ of integration on a background field $1^{\circ} .5$ west of the cD NGC 1399. The image scale was $0.158 \mathrm{pix}^{-1}$; however, the chip was slightly vignetted around the edges, and we shifted the telescope $\sim 6^{\prime \prime}$ between individual exposures, so the final field size which received the full integration time was about 5 '. $1 \times 5$ ' 1 . We processed the images as described by Ajhar, Blakeslee, \& Tonry (1994) and Blakeslee \& Tonry (1995; hereafter BT95). The seeing in the final NGC 1399 image was 0.94 , while the seeing in the other images ranged from $1^{\prime \prime} .03$ to $1^{\prime \prime} .05$. The photometry was calibrated using Landolt (1992) standard stars; there is no detectable Galactic extinction in the directions of these galaxies (Burstein \& Heiles 1984).

After subtracting smooth models of the galaxy surface brightness profiles, we used a version of the program DoPHOT (Schechter, Mateo, \& Saha 1993) for the point source photometry. Completeness corrections were determined by scaling and adding grids (so as to avoid artificial crowding) of $32 \times 32$ "cloned" PSF stars and then finding them again with DoPHOT. The scaling was done at 0.4 mag intervals; we 
interpolated to find the completeness corrections at intermediate magnitudes. The images were then divided up into three radial regions: 20-40", 40-80", and 80-160". Since the surface density of GCs falls off roughly as $r^{-2}$ (Harris 1991; the density for NGC 1399 goes more like $r^{-1.5}$ ), these regions contain comparable numbers of GCs. We settled on a cutoff magnitude $m_{c}$ of $V=24.4$ for the innermost region in each galaxy; at this magnitude, the completeness levels for this region ranged from $77 \%$ to to $88 \%$. For the intermediate region, the completeness levels were in the same range at $V=24.8$, so we used this for the cutoff magnitude. For simplicity, we also used $V=24.8$ for $m_{c}$ in the outermost region, although the completeness levels for this region ranged from $90 \%$ to $96 \%$ at this magnitude. The photometric error is typically $\sim 0.12 \mathrm{mag}$ at $V=24.8$. Any objects classified by DoPHOT as extended were excluded from further analysis. Objects brighter than $m_{c}$ in each region were then binned in magnitude and our completeness corrections were applied. We subtracted the completeness-corrected luminosity function of the unresolved objects in the background field from the luminosity functions of the objects in the program fields to produce the final GCLFs presented below.

After removing all objects brighter than $m_{c}$ in each region, we measured the PSFconvolved variance, or fluctuations, remaining in the image. Our variance analysis method is described in detail by Tonry et al. (1990). The variance measurement effectively acts as an "extra bin" in fitting the GCLF. BT95 demonstrate how this measurement can be used for deriving GCLF parameters. The conversion from measured variances to GC densities is done in the same way here, but the GC counts are treated differently in that they are binned for more GCLF shape information. In addition, we leave $m_{V}^{0}$ as a free parameter, instead of varying it only within some presupposed acceptable range, as in BT95. A more detailed account of our point source photometry and positions, completeness experiments, and fluctuation measurements will be provided elsewhere (Blakeslee et al. 1997) 

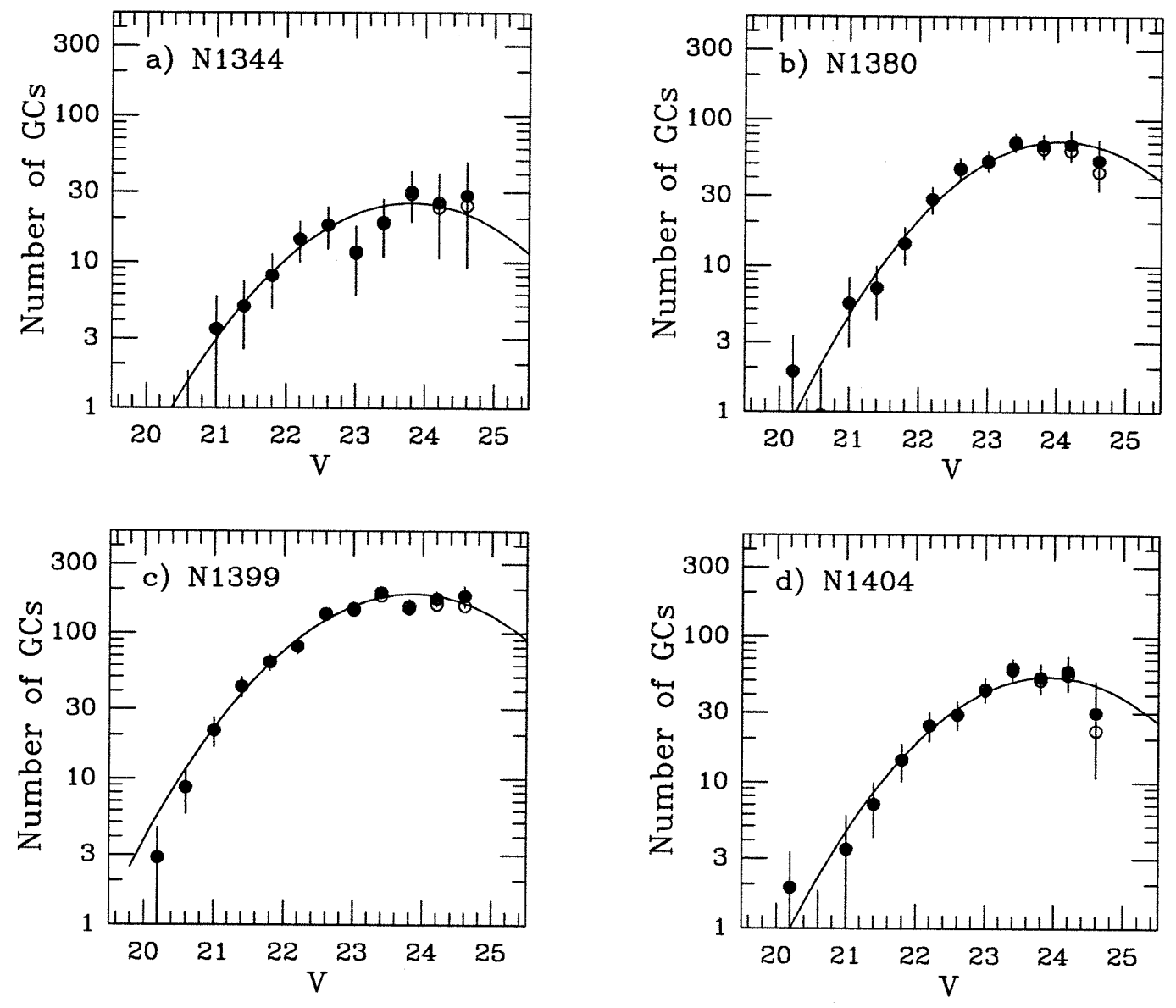

Figure 3-1: The $V$-band globular cluster luminosity functions of the four Fornax galaxies. Filled symbols represent the final counts following incompleteness and background corrections. Open symbols show what the counts would be with background subtraction, but no incompleteness corrections. Errorbars represent the uncertainties in our corrections and the Poisson errors in the counts. 


\subsection{Results}

We defer the analysis of the spatial structure and total sizes of the GC populations until a future paper and concentrate on the luminosity functions. We also defer any further discussion of our observations of GCs in the giant disturbed galaxy NGC 1316 (Fornax A). The GCLF of this galaxy was not well fitted by a Gaussian model $\left(\chi_{N}^{2} \gtrsim 3\right.$ instead of $\sim 1$ ) and would require a more thorough analysis than we provide here.

\subsubsection{The GCLF in Fornax}

Figure 3-1 presents the $V$-band GCLFs of the four Fornax galaxies. The plotted curves are Gaussians having $\sigma$ and $m_{V}^{0}$ values which are the weighted means of the values found in our three analysis regions of each galaxy. The values were derived in the separate regions by $\chi^{2}$ minimizations using the counts brighter than the individual cutoff magnitudes and our variance measurements. For purposes of displaying the GCLFs, however, we used $m_{c}=24.8$ everywhere, applied our completeness corrections, then binned the regions all together.

Table 3.1 lists our final values for the GCLF parameters; they are nearly identical within the errors. The columns are: galaxy name, Hubble type and total apparent $V$-band magnitude (both from de Vaucouleurs et al. 1991), Gaussian dispersion of the GCLF, $V$-band GCLF turnover magnitude, and total number of GCs which went into the Gaussian fits (following corrections for incompleteness and background). The cD NGC 1399 is the only one of these galaxies with a previously well-studied GCLF. Geisler \& Forte (1990) found $m_{V}^{0}=23.45$ for this galaxy, but assumed $\sigma=1.20 \mathrm{mag}$ (these parameters are correlated when the limiting magnitude is near the turnover). Bridges, Hanes, \& Harris (1991) used $\sigma \approx 1.40$, and found $m_{V}^{0}=23.85 \pm 0.30$, in close agreement with our value.

As weighted means of the Fornax GCLF parameters, we take $\langle\sigma\rangle_{F o r}=1.35 \pm$ $0.07 \mathrm{mag} ;\left\langle m_{V}^{0}\right\rangle_{\text {For }}=23.88 \pm 0.10$. The dispersion in $m_{V}^{0}$ for this admittedly small sample is just $0.1 \mathrm{mag}$, which, considering the measurement errors, can be taken as a very strong upper limit to the intrinsic variation in $M_{V}^{0}$ among these galaxies. 
Table 3.1: Fornax GCLF Parmeters

\begin{tabular}{ccrccccrc}
\hline \hline Galaxy & Type & \multicolumn{1}{c}{$V_{T}$} & $\sigma$ & \pm & $m_{V}^{0}$ & \pm & N & \pm \\
\hline NGC 1344 & E5 & 10.35 & 1.35 & 0.18 & 23.80 & 0.25 & 140 & 20 \\
NGC 1380 & S0 & 9.91 & 1.30 & 0.17 & 24.05 & 0.25 & 375 & 35 \\
NGC 1399 & E1/cD & 9.57 & 1.38 & 0.09 & 23.83 & 0.15 & 1120 & 45 \\
NGC 1404 & E1 & 9.98 & 1.32 & 0.14 & 23.92 & 0.20 & 300 & 30 \\
\hline \hline
\end{tabular}

\subsubsection{Comparison with Virgo}

The largest CCD study to date of GC populations in Virgo was by Ajhar, Blakeslee, \& Tonry (1994). In that paper, we were primarily concerned with the $V R I$ colors of the GCs around ten Virgo and Leo ellipticals, but we also examined the $R$-band GCLFs of the five most populous Virgo systems. We concentrated on $R$ because the data went the deepest in that band, although they did not reach beyond the turnover. For that reason and because there were no background fields, no attempt was made to fit for the GCLF parameters. However, we were able to conclude that for a fixed Gaussian width, $M_{R}^{0}$ could not vary by more than $0.30 \mathrm{mag}$. This conclusion was based mainly on the fact that the larger SBF distance to NGC 4406 accounted for its apparently fainter GCLF. Figure 3-2 reproduces the relevant plot from Ajhar et al.

The NGC 4365 GCLF was offered in that paper as an unresolved puzzle, as it did not match our shifted Gaussians very well. More recently, HST imaging (Forbes 1996) has shown than the GCLF of NGC 4365 is well described by the Virgo core GCLF shifted to the distance indicated by SBF. Using this result and others from the literature, Harris (1996) finds that $M_{V}^{0}$ has an rms dispersion of $0.21 \mathrm{mag}$ (some of which is due to measurement error) among five Virgo ellipticals. If NGC 4636 is excluded from his list, the dispersion in $M_{V}^{0}$ drops to $0.11 \mathrm{mag}$, similar to what we found above for Fornax. In fact, NGC 4636 may not be at the distance of the Virgo core, since its projected distance places it on the outskirts of the cluster nearly $3 \mathrm{Mpc}$ from M87, exactly the offset in radial distance required to match its turnover to that of the other ellipticals. Tully (1987b) and Faber et al. (1989) both place it outside 

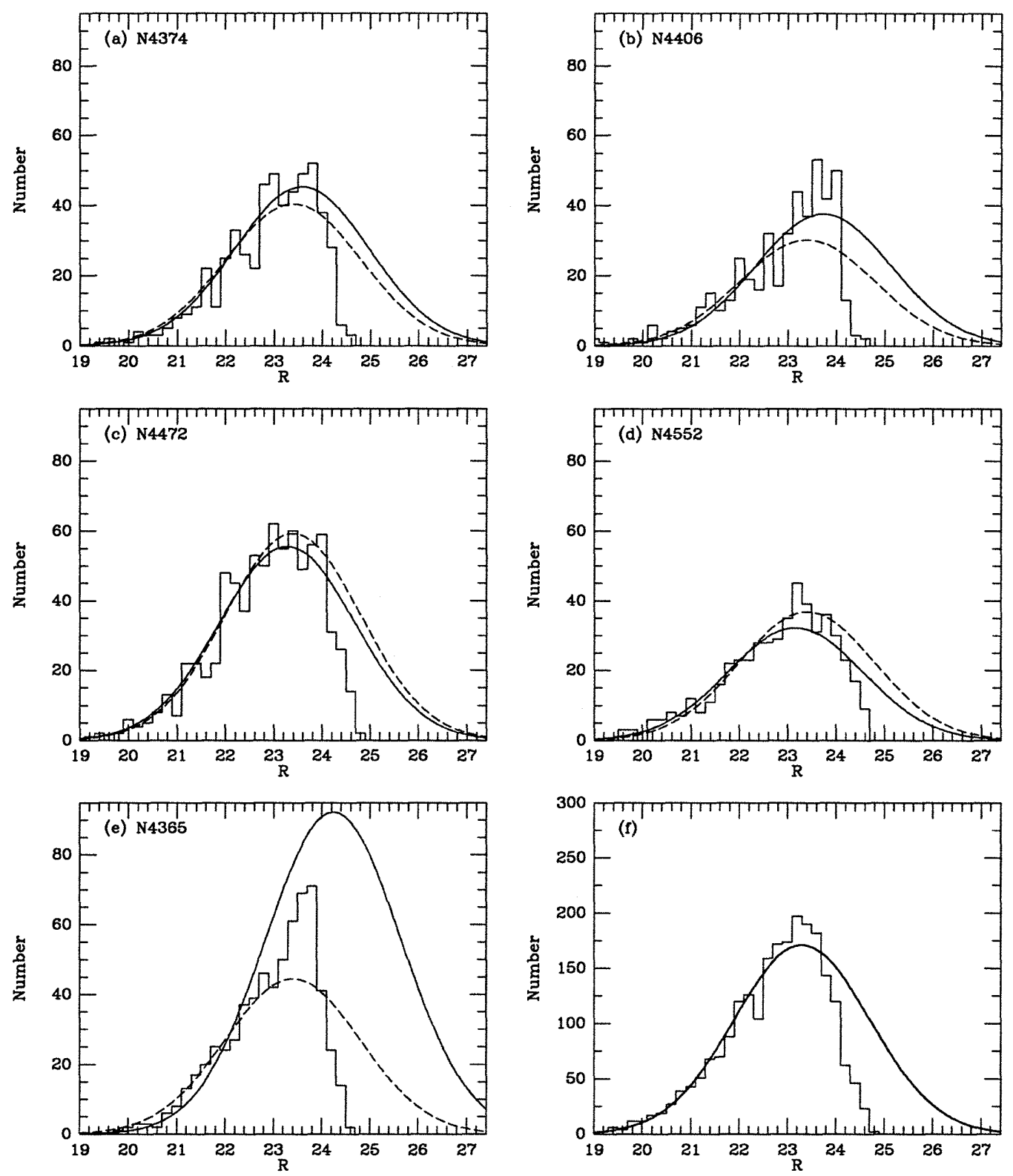

Figure 3-2: The $R$-band luminosity functions for the five largest Virgo galaxies in the Ajhar et al. sample. The histograms represent raw counts of all point sources in the fields. Solid curves are Gaussians with $\sigma=1.4$ and means that were fixed according to the relative SBF distances of the galaxies and the assumed value $m_{R}^{0}=23.40$ for the Virgo core; thus, the individual means are (a) 23.58, (b) 23.73, (c) 23.28, (d) 23.16, (e) 24.24, and (f) 23.30. The dashed curves, plotted for comparison, all use $m_{R}^{0}=23.40$. The histogram in (f) is the sum of those in (a) through (d), with (a) and (b) shifted forward by 1 and 2 bins, respectively. Curve normalizations were fitted in each case from $2.5 \sigma$ brighter than the adopted means to $R=23$, except for (f), where the fit was done to $R=22.8$. Beyond this limit, the competing effects of incompleteness and contamination become significant. 
of Virgo proper. We conclude that among Virgo galaxies $M_{V}^{0}$ is constant to within $0.2 \mathrm{mag}$, and maybe to within $0.1 \mathrm{mag}$.

The most precise determination of the GCLF turnover in Virgo was by Whitmore et al. (1995), who found $m_{V}^{0}=23.72 \pm 0.06$ for M87. Averaging this with the values for NGC 4472 and NGC 4649 (Secker \& Harris 1993, based on the data of Harris et al. 1991) and using $(B-V)=0.75 \pm 0.05$ (Couture, Harris, \& Allwright $1990,1991)$, we find $\left\langle m_{V}^{0}\right\rangle_{V i r}=23.75 \pm 0.05$. Thus, assuming a universal $M_{V}^{0}$ yields $\Delta(m-M)=0.13 \pm 0.11$ for the relative Fornax-Virgo distance modulus. We have left NGC 4365 (Secker \& Harris; Forbes 1996) and NGC 4636 (Kissler et al. 1994) out of the Virgo average because they are both well outside the Virgo core; including them would serve to drop the relative Fornax-Virgo modulus.

\subsubsection{Does $M^{0}$ Depend on Environment?}

We now forsake the assumption of universality and compare the Fornax GCLF with those observed elsewhere. To do this, we need a self-consistent set of independent distance determinations to galaxies or groups in which $m_{V}^{0}$ has been measured. We start by fixing $(m-M)=31.0$ as the Virgo distance modulus; this is both the Jacoby et al. (1992) value and the latest HST Cepheid Key Project result (Ferrarese et al. 1996). For the relative Fornax-Virgo distance modulus, we take $\Delta(m-M)=$ $0.25 \pm 0.08$ from an average of the PNLF, SBF, $D_{n}-\sigma$, SN Ia, and Tully-Fisher methods (Ciardullo et al. 1993; Faber et al. 1989; Riess 1996; Willick 1996).

We use measurements of $m_{V}^{0}$ for M31 (Secker 1992), M81 (Perelmuter \& Racine 1995), the Leo group ellipticals (Harris 1990), and the HST limit on $m_{V}^{0}$ for NGC 4881 in Coma (Baum et al. 1995a). The Cepheid distance moduli to M31 and M81 are $24.43 \pm 0.10$ and $27.80 \pm 0.20$, respectively (Freedman \& Madore 1990; Freedman et al. 1994). This M31 distance modulus is the proper one to use, as it is the one assumed by Jacoby et al. (1992) and is consistent with the more recent HST Cepheid distances. For the Leo group, we average the HST Cepheid distance to the spiral M96 (Tanvir et al. 1995) with the PNLF and SBF distances to the ellipticals (Ciardullo et al. 1993) and get $(m-M)=30.2 \pm 0.13$. The relative Virgo-Coma distance 


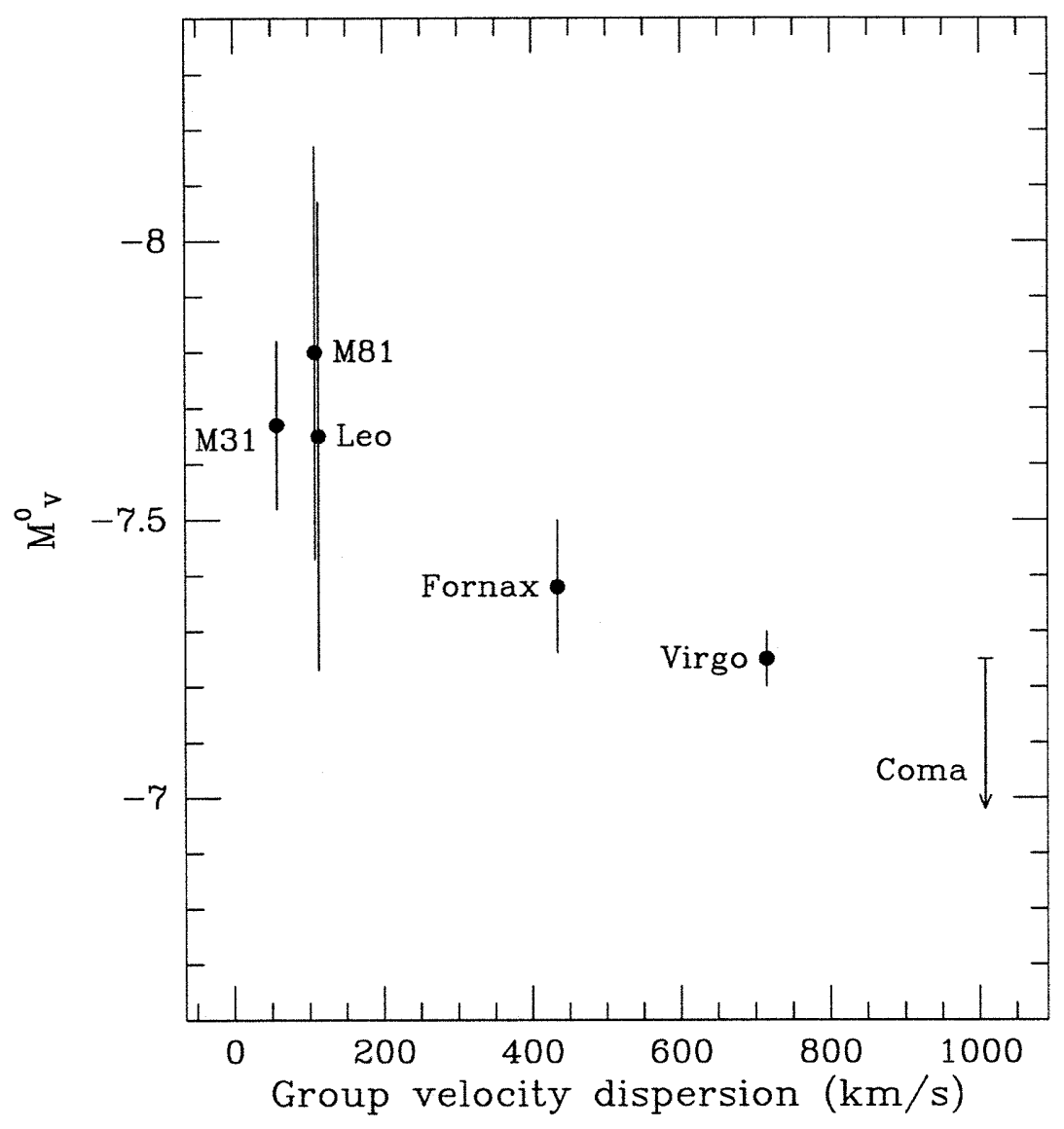

Figure 3-3: The GCLF turnover magnitude $M_{V}^{0}$ plotted against the velocity dispersion of the host galaxy's environment, used as a measure of the local density. See text for details.

modulus is well determined at $\Delta(m-M)=3.71 \pm 0.10$ (e.g. van den Bergh 1992; de Vaucouleurs 1993; Whitmore et al. 1995). Finally, in an effort to preserve neutrality in the controversy over the RR Lyrae calibration (e.g. van den Bergh 1995, Walker 1992), we omit the Milky Way from our discussion, remarking only that recent MW $M_{V}^{0}$ values have ranged from $-7.29 \pm 0.13$ (Secker 1992) to $-7.60 \pm 0.11$ (Sandage \& Tammann 1995; see also the discussion by Baum et al. 1995a).

Figure 3-3 shows the resulting $M_{V}^{0}$ values plotted against the velocity dispersions of the groups and clusters, from Tully (1987a) and Zabludoff, Huchra, \& Geller (1990). We use velocity dispersion as the most convenient indicator of the depth of the local potential well; it closely correlates with Tully's estimated group densities and with cluster richness. There is a trend of decreasing turnover luminosity with increasing local density. The offset in $M_{V}^{0}$ between the small groups and Fornax/Virgo is 0.4 mag. 
The use of the straight Cepheid distance to Leo would move its $M_{V}^{0}$ brighter by $0.1 \mathrm{mag}$, further away from the other ellipticals; the inclusion of NGC 4365 and NGC 4636 would move the Virgo $M_{V}^{0}$ fainter. In addition, the preliminary result $m_{V}^{0} \approx 28.0$ $\left(M_{V}^{0} \approx-6.7\right)$ for IC 4051 (Baum et al. 1995b) indicates that there may be another $\sim 0.5$ mag offset in $M_{V}^{0}$ for the very rich Coma cluster. Thus, we believe we are seeing real evidence for an environmental dependence of $M_{V}^{0}$.

\subsection{Discussion}

We have found that the GCLF is remarkably constant within the Fornax cluster, but, as Figure 3-3 shows, may vary with environment. Ashman et al. (1995) suggested that metallicity differences result in $M_{V}^{0}$ values which are systematically brighter by $\sim 0.15$ mag for spirals. Large ellipticals usually do have higher metallicity GC populations; however, NGC 4881 in Coma has a GC color/metallicity distribution similar to that of the MW (Baum et al. 1995a), yet its $M_{V}^{0}$ is very faint. In addition, the Leo elliptical NGC 3379, with its relatively high metallicity GC population (Ajhar, Blakeslee \& Tonry 1994), has an exceedingly bright $M_{V}^{0}$, though with a large uncertainty (Harris 1990). Finally, we note that the magnitude of the environmental effect we propose is a factor of 3-6 larger than the Ashman et al. $M_{V}^{0}$ metallicity shift.

We suggest that the most straightforward way to produce the present-day nearGaussian GCLF is to assume that two simultaneous and competing effects were operating when GCs formed: a "creation" process which preferentially created low-mass GCs, cutting the mass function off at the high end, and a "destruction" process which inhibited the formation of, or quickly destroyed, low-mass GCs. If each process operated in a manner which was independent of the details of the environment, then the final mass (luminosity) function would be universal, but if one depended more sensitively on environment than did the other, the final mass function would vary. This situation is schematically illustrated in Figure 3-4, where we use $S_{N}$ for the GC "specific frequency" (number of GCs per unit luminosity of the host galaxy). In Figure 3-4a, we assume that the creation process is relatively universal, but that the 


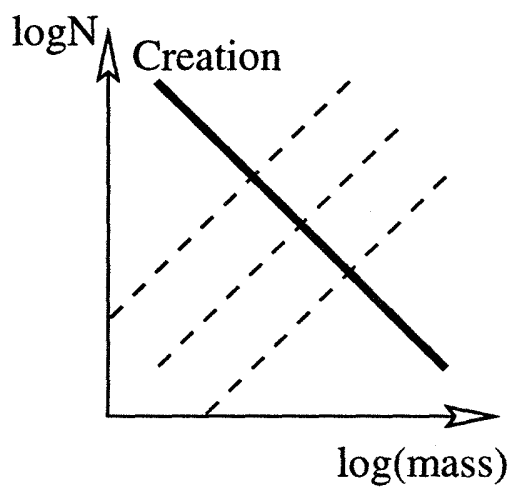

(a)
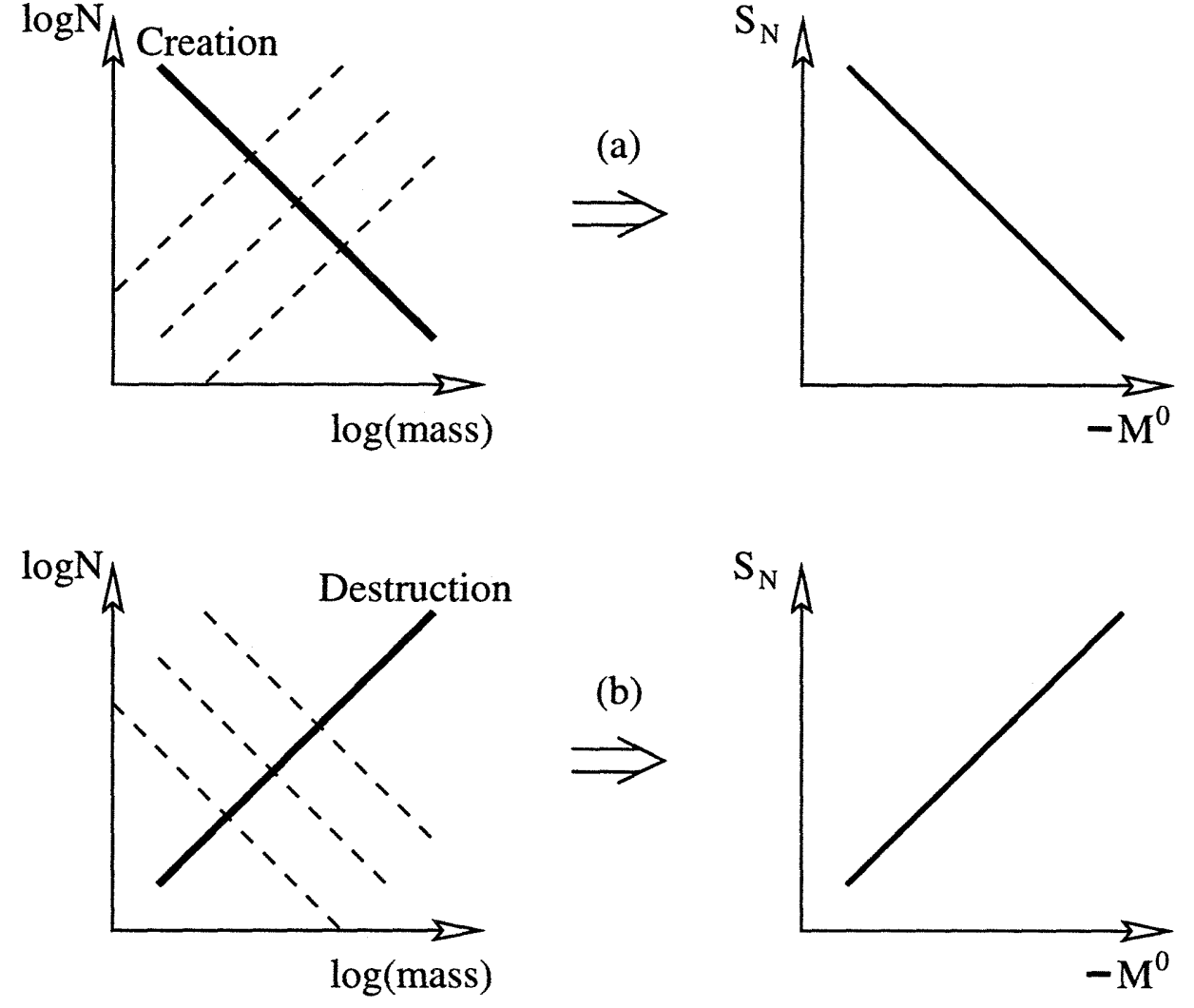

Figure 3-4: The effects of variable GC creation/de-struction mechanisms. In part (a) the GC creation process, shown as a power-law growing to smaller mass, is universal (dark solid line), and the destruction process, a power law which wipes out low-mass objects, varies with environment (dashed lines). Since the total number of GCs is the integral under the intersecting creation/destruction lines, this situation results in an anti-correlation between GC specific frequency $S_{N}$ and GC mean logarithmic luminosity $-M^{0}$. In part (b) the creation process (dashed lines) varies with environment, while the destruction process (dark solid line) is universal. The result here is a positive correlation between $S_{N}$ and $-M^{0}$.

destruction process varies. This leads to $M^{0}$ being variable, and predicts an inverse correlation between the number of GCs formed and their mean brightness. On the other hand, if the destruction process is constant and the creation process is more variable we will again get a variable $M^{0}$, but with a direct correlation between the number of GCs and their luminosity, as shown in Figure 3-4b.

Empirically, we think we see evidence for the latter sort of behavior among "coeval" galaxies, i.e., those located within the same physical association. In Virgo, for instance, M87 has a very large $S_{N}$ and a slightly brighter $M^{0}$ than its close neighbors, and similarly in Fornax for NGC 1399. In this context, the GCLF would depend on 
the extent to which the host galaxy dominated its local environment. On the other hand, the main point of this paper is that we see the former behavior among very "heterogeneous" systems of galaxies. Young groups dominated by spirals have fewer GCs than galaxy clusters such as Virgo, which in turn may have fewer than rich clusters such as Coma, and we find that the central luminosity of the GCLF is declining along this sequence.

As an example of how such an interplay of opposing processes might work in practice, we consider the common picture of structure formation through gravitational instability (e.g. Peebles 1993). Here, the "creation" process is the primordial spectrum of density fluctuations which favors low-mass clusters, and the "destruction" process is the inhibition of the collapse of low-mass objects resulting from the Jeans mass. In this picture, the Jeans mass can be a very rapidly growing function of time (Tegmark et al. 1996), and the densest systems of galaxies, forming first, would have experienced a less restrictive low-mass cutoff and hence have more, and fainter, GCs. This is precisely the case depicted in Figure 3-4a.

Harris \& Pudritz (1994) have proposed a detailed astrophysical theory of GC formation which is perhaps more illustrative of the "coeval" case of Figure 3-4b. They suggest that the "creation" process is made more efficient by the larger external pressures of dense environments. Their "destruction" process is the tidal disruption and evaporation of low-mass GCs, and this might be less sensitive to environment (although see Murali \& Weinberg 1996). Of course, if the cutoff is very abrupt (a steep "destruction" line), then $M^{0}$ will not correlate very strongly with $S_{N}$.

We do not have better "creation" and "destruction" processes to offer than have been advanced elsewhere, but we believe that this description is a profitable way to frame the discussion. Until now, the assumed constancy of $M^{0}$ has been a serious obstacle to reasonable models for GC formation, so we conclude by re-emphasizing our primary point. The GCLF apparently does depend on environment, with $M_{V}^{0}$ being fainter in denser regions, although it may be remarkably constant within a single group of galaxies. This dependence will present challenges for the use of the GCLF as a distance indicator. For instance, it is clear that the method cannot be 
properly calibrated from Local Group observations; however, if applied consistently within similar environments, it should provide good relative distances. Moreover, the door is now open to correlations between $M^{0}$ and $S_{N}$, and $M^{0}$ and environment, which may yield valuable insights into the conditions and processes which prevailed at the time of GC/galaxy formation.

\subsection{References}

Ajhar, E. A., Blakeslee, J. P., \& Tonry, J. L. 1994, AJ, 108, 2087

Ashman, K. M., Conti, A., \& Zepf, S. E. 1995, AJ, 110, 1164

Baum, W. A. et al. 1995a, AJ, 110, 2537

Baum, W. A., Hammergren, M., Groth, E. J., Faber, S. M., Grillmair, C. J., \& Ajhar, E. A., 1995b, BAAS, 27, 1407, poster presented 17 January 1996 at the 187th AAS meeting

Blakeslee, J. P. \& Tonry, J. L. 1995, ApJ, 442, 579 (BT95)

Blakeslee, J. P. et al. 1997, in preparation

Bridges, T. H., Hanes, D. A., \& Harris, W. E. 1991, AJ, 101, 469

Burstein, D. \& Heiles, C. 1984, ApJS, 54, 33

Ciardullo, R., Jacoby, G. H., \& Tonry, J. L. 1993, ApJ, 419, 479

Couture, J., Harris, W. E., \& Allwright, J. W. B. 1990, ApJS, 73, 671

Couture, J., Harris, W. E., \& Allwright, J. W. B. 1991, ApJ, 372, 97

de Vaucouleurs, G. 1993, ApJ, 415, 10

de Vaucouleurs, G., de Vaucouleurs, A., Corwin, H. G., Jr., Buta, R. J., Paturel, G., \& Fouqué, P. 1991, Third Reference Catalog of Bright Galaxies (New York: Springer)

Faber, S. M., Wegner, G., Burstein, D., Davies, R. L., Dressler, A., Lynden-Bell, D., \& Terlevich, R. J. 1989, ApJS, 69, 763

Ferrarese, L. et al. 1996, ApJ, 464, 568

Forbes, D. A. 1996, AJ, 112, 954

Freedman, W. L. et al. 1994, ApJ, 427, 628 
Freedman, W. L. \& Madore, B. F. 1990, ApJ, 365, 186

Geisler, D. \& Forte, J. C. 1990, ApJ, 350, L5

Harris, W. E. 1990, PASP, 102, 966

Harris, W. E. 1991, ARA\&A, 29, 543

Harris, W. E. 1996, poster presented May 1996 at the STScI Symposium on the Extragalactic Distance Scale

Harris, W. E., Allwright, J. W., Pritchet, C. J., \& van den Bergh, S., 1991, ApJS, 76,115

Harris, W. E. \& Pudritz, R. E. 1994, ApJ, 429, 177

Jacoby, G. H. et al. 1992, PASP, 104, 599

Kissler, M., Richtler, T., Held, E. V., Grebel, E. K., Wagner, S. J., \& Capaccioli, M. 1994, A\&A, 287, 463

Landolt, A. U. 1992, AJ, 104, 340

Murali, C. \& Weinberg, M. D. 1996, MNRAS, in press

Peebles, P. J. E. 1993, Principles of Physical Cosmology (Princeton: Princeton Univ. Press)

Perelmuter, J.-M. \& Racine, R. 1995, AJ, 109, 1055

Riess, A. G. 1996, PhD Thesis, Harvard University

Sandage, A. \& Tammann, G. A. 1995, ApJ, 446, 1

Schechter, P. L., Mateo, M., \& Saha A. 1993, PASP, 105, 1342

Secker, J. 1992, AJ, 104, 1472

Secker, J. \& Harris, W. E. 1993, AJ, 105, 1358

Tanvir, N. R., Shanks, T., Ferguson, H. C., \& Robinson, D. R. T. 1995, Nature, 377, 27

Tegmark, M., Silk, J., Rees, M. J., Blanchard, A., Abel, T., \& Palla, F. 1996, ApJ, in press

Tonry, J. L. 1991 ApJ, 373, L1

Tonry, J. L., Ajhar, E. A., \& Luppino, G. A. 1990, AJ, 100, 1416

Tully, R. B. 1987a, ApJ, 321, 280

Tully, R. B. 1987b, Nearby Galaxies Catalog (Cambridge: Cambridge Univ. Press) 
van den Bergh, S. 1992, PASP, 104, 861

van den Bergh, S. 1995, ApJ, 446, 39

Walker, A. R., ApJ, 390, L81

Whitmore, B. C., Sparks, W. B., Lucas, R. A., Macchetto, F. D., \& Biretta, J. A. 1995, ApJ, 454, L73

Willick, J. 1996, private communication

Zabludoff, A. I., Huchra, J. P., \& Geller, M. J. 1990, ApJS, 74, 1 


\section{Chapter 4}

\section{Globular Clusters in Abell Clusters: Sample Selection, Observations, and Reductions}

\subsection{The BCG Sample}

The galaxy sample used in this study was selected from the Lauer \& Postman (1994; hereafter referred to as LP) volume-limited survey of brightest cluster galaxies (BCGs) in 119 Abell and ACO galaxy clusters (Abell 1958; Abell, Corwin, \& Olowin 1989). The LP sample includes nearly all the Abell/ACO clusters with redshift $c z<15,000$ $\mathrm{km} \mathrm{s}^{-1}$ and galactic latitude $|b|>15^{\circ}$. There were 7 clusters excluded from their survey because of spiral, starforming irregular, or anomalously faint BCGs, 6 "clusters" which LP found were not true galaxy overdensities, and 2 clusters for which they were unable to obtain the necessary data. Postman \& Lauer (1995; hereafter PL) present and discuss the data used by LP, including observations of second brightest, or second ranked, galaxies (SRGs) in clusters for which the magnitude offset between first and second was small.

To ensure that reasonably accurate statistical statements about GCSs would result, I selected for this study as complete a subsample as possible from the LP survey. The final sample presented here includes all of the northern hemisphere (thus, no ACO clusters) LP BCGs and SRGs with $c z<10,000 \mathrm{~km} \mathrm{~s}^{-1}$ and $\alpha>0.4$, where 
$\alpha \equiv d \log L /\left.d \log r\right|_{r_{m}}$, is the logarithmic slope of the galaxy luminosity evaluated at the metric radius $r_{m}=10 h^{-1} \mathrm{Mpc}$. (Larger $\alpha$ implies a more extended galaxy; it was first introduced by Hoessel [1980], who called it the "structure parameter" of the galaxy profile). In addition, NGC 4839 in Coma has been added to this sample because it is a known $\mathrm{cD}$ galaxy, though not the first or second brightest Coma member. (A cD galaxy is a giant elliptical that possesses an extended, low surface brightness halo; thus, its radial profile exhibits a significant excess of light relative to an $r^{1 / 4}$ law. See Tonry [1987] for a review.) Thus, the sample studied here comprises 23 galaxies in 19 Abell clusters. Of the nine true clusters omitted by LP, only A426 (the Perseus cluster, with its giant disturbed BCG) and A400 ("anomalously faint" BCG) are within the above redshift and coordinate cutoffs. The Perseus BCG is not well suited to this study due to all the dust and recent star formation, but the multiple nucleus BCG in A400 might be a good target for future investigation.

The "alpha cutoff" employed here excludes only 2 BCGs (in A189 and A1267) which would otherwise have been included. In fact, I had intended to observe these two galaxies as well, but due to the finite quality of observing time, some prioritization was necessary. The prioritization was based mainly on distance; these two galaxies, both near the redshift limit of our sample, were given the lowest priorities for the following reasons. The A189 BCG is quite faint in apparent magnitude, and is the only northern LP galaxy within this redshift range that has an $R$-band absolute metric magnitude $M_{R}>-22$ (LP, Table 3). The A1267 BCG, also faint, shows a significant amount of dust and spiral structure, suggesting that LP would likewise have excluded it from their sample if they had had the benefit of deeper images. I was able to obtain over an hour of integration time on this galaxy, but much more would have been required. Afterwards, I discovered that my qualitative impression of these galaxies as being too "small" to yield much information on their GCSs was quantified by the fact that they both have $\alpha<0.34$, while the $\sim 20$ other galaxies selected for this study all have $\alpha \gtrsim 0.43$.

Table 4.1 gives a listing of the galaxies in the present sample. The columns are: galaxy identification (Abell number of, and galaxy rank in, cluster); right ascen- 
Table 4.1: The BCG Sample

\begin{tabular}{|c|c|c|c|c|c|c|c|c|c|}
\hline Abell & \multicolumn{2}{|c|}{ R.A. (J2000) Dec. } & $l$ & $b$ & $c z_{h}$ & $A_{B}$ & $M_{R, m}$ & $\alpha$ & Name \\
\hline $262-1$ & 015246 & +360905 & 136.57 & -25.09 & 4831 & .24 & -22.189 & .810 & N0708 \\
\hline $347-1$ & 022526 & +414927 & 141.11 & -17.71 & 5257 & .24 & -22.352 & .601 & N0910 \\
\hline $397-1$ & 025629 & +155459 & 161.81 & -37.34 & 10286 & .27 & -22.542 & .582 & C463-037 \\
\hline $539-1$ & 051655 & +063310 & 195.65 & -17.60 & 9682 & .51 & -22.484 & .511 & C421-019 \\
\hline $539-2$ & 051637 & +062628 & 195.71 & -17.72 & 8318 & .51 & -22.210 & .785 & U03274 \\
\hline $569-1$ & 070908 & +483655 & 168.58 & +22.80 & 5724 & .34 & -22.418 & .486 & N2329 \\
\hline $634-1$ & 081545 & +581916 & 159.06 & +33.79 & 8135 & .13 & -22.258 & .498 & U04289 \\
\hline $779-1$ & 091947 & +334459 & 191.09 & +44.39 & 6867 & .00 & -22 & .594 & $\mathrm{~N} 2832^{\dagger}$ \\
\hline $999-1$ & 102324 & +125006 & 227.94 & +52.58 & 9749 & .11 & -22.267 & .441 & C065-015 \\
\hline $1016-1$ & 102708 & +1100 & 231.2 & 52.48 & 9705 & .03 & -22 & .430 & \\
\hline $1177-1$ & 110944 & +214532 & 220.44 & +66.29 & 9561 & .00 & -22 & .724 & U06203 \\
\hline $1185-1$ & 111038 & +284603 & 202.8 & +67.72 & 10521 & .00 & -22.444 & .616 & N3550 \\
\hline $1314-1$ & 113449 & +490438 & 151.77 & +63.54 & 9977 & .00 & -22.461 & .583 & I0712 \\
\hline $1367-1$ & 114402 & +195659 & 234.29 & +72.99 & 6237 & .00 & -22 & .518 & N3842 \\
\hline $1656-1$ & 130008 & +275836 & 57.1 & -87.89 & 6497 & .05 & -22.957 & .590 & N4889 \\
\hline $1656-2$ & 125936 & +275733 & 58.08 & +88.01 & 7176 & .05 & -22.545 & .855 & $\mathrm{~N} 4874^{\dagger}$ \\
\hline $1656-3$ & 125725 & +272948 & 48.7 & +88.62 & 7335 & .03 & -22.287 & .610 & N4839 ${ }^{\dagger}$ \\
\hline $2162-1$ & 161236 & +292904 & 48.33 & +46.01 & 9547 & .07 & -22.475 & .503 & $\mathrm{~N} 6086^{\dagger}$ \\
\hline $2197-1$ & 162945 & +404842 & 64.68 & +43.51 & 8800 & .00 & -22.887 & .586 & N6173 \\
\hline $2197-2$ & 162741 & +405537 & 64.8 & -43.90 & 9408 & .01 & -22.350 & .702 & N6160* \\
\hline 2199-1 & 162838 & +393303 & 62.93 & +43.69 & 9348 & .00 & -22.657 & .755 & $\mathrm{~N} 6166^{\dagger}$ \\
\hline $2634-1$ & 233829 & +270150 & 103.50 & -33.07 & 9141 & .16 & -22.748 & .650 & N7720 ${ }^{\dagger}$ \\
\hline $2666-1$ & 235059 & +270848 & 106.7 & -3381 & 8123 & .13 & -22.768 & .549 & N7768 \\
\hline
\end{tabular}

${ }^{\dagger}$ Schombert (1988) $\mathrm{cD}$ galaxy.

${ }^{*}$ Selected as BCG by Hoessel, Gunn, \& Thuan (1980).

sion and declination (J2000 coordinates); galactic longitude and latitude; heliocentric velocity in $\mathrm{km} \mathrm{s}^{-1} ; B$-band extinction (as listed by Burstein \& Heiles 1984); $R$-band absolute metric magnitude and $\alpha$ parameter (both as listed in LP, except for the SRGs, whose values come from PL, and A1656-3, for which the values were determined from the data presented here); and the "common name" for each galaxy. The common galaxy name was chosen first to be the NGC or IC number (Dreyer 1888, 1908; prefixed by N or I, respectively), then the UGC number (Nilson 1973; prefixed by U), and last by the CGCG number (Zwicky 1968; prefixed by C). The table also notes the galaxies found by Schombert (1988) to possess cD envelopes. 
Table 4.2: Abell Cluster Information

\begin{tabular}{rcccccccccc}
\hline \hline Abell & $\mathrm{RA}_{X}(\mathrm{~J} 2000) \operatorname{Dec}_{X}$ & $c z_{h}$ & $c z_{C}$ & $c z_{A}$ & $\sigma$ & $T_{X}$ & $\mathrm{R}$ & $\mathrm{BM}$ & $\mathrm{RS}$ \\
\hline 262 & 15246 & +360836 & 4913 & 4659 & 5310 & 498 & 2.4 & 0 & III & $\mathrm{C}$ \\
347 & $\ldots$ & $\ldots$ & 5604 & 5391 & 6000 & 582 & $\ldots$ & 0 & II-III & $\mathrm{C}$ \\
397 & 25638 & +155338 & 9975 & 9765 & 10560 & $\ldots$ & $1.6^{*}$ & 0 & III & $\mathrm{F}$ \\
539 & 51636 & +062630 & 8754 & 8755 & 9390 & 787 & 3.0 & 1 & III & $\mathrm{F}$ \\
569 & 70911 & +483658 & 5749 & 5832 & 6060 & 374 & $1.4^{*}$ & 0 & II: & $\mathrm{B}$ \\
634 & 81434 & +580252 & 8135 & 8234 & 8280 & 309 & $0.9^{\dagger}$ & 0 & III & $\mathrm{F}$ \\
779 & 91947 & +334449 & 6796 & 7039 & 6930 & 472 & $1.5^{*}$ & 0 & I-II: & $\mathrm{cDp}$ \\
999 & $102323+125013$ & 9603 & 9942 & 9600 & 417 & $1.2^{*}$ & 0 & II-III: & $\mathrm{L}$ \\
1016 & $102703+105842$ & 9669 & 10013 & 9660 & 247 & $1.3^{\dagger}$ & 0 & $\ldots$ & $\mathrm{L}$ \\
1177 & $\ldots$ & $\ldots$ & 9561 & 9885 & 9420 & $\ldots$ & $\ldots$ & 0 & I & $\mathrm{cD}$ \\
1185 & $111045+284246$ & 9917 & 10217 & 9780 & 718 & 3.9 & 1 & II & $\mathrm{C}$ \\
1314 & $113448+490510$ & 9838 & 10043 & 9690 & $\ldots$ & 5.0 & 0 & III & $\mathrm{C}$ \\
1367 & $114440+194235$ & 6469 & 6795 & 6240 & 802 & 3.5 & 2 & II-III: & $\mathrm{F}$ \\
1656 & $125943+275612$ & 6961 & 7229 & 6570 & 1140 & 8.1 & 2 & II & $\mathrm{B}$ \\
2162 & $\ldots$ & $\ldots$ & 9629 & 9689 & 9030 & $\ldots$ & $\ldots$ & 0 & II-III & I \\
2197 & $162740+405539$ & 9042 & 9065 & 8520 & 589 & $1.6^{*}$ & 1 & III & $\mathrm{L}$ \\
2199 & 162838 & +393310 & 9034 & 9059 & 8490 & 823 & 4.7 & 2 & I & $\mathrm{cD}$ \\
2634 & 233825 & +270056 & 9153 & 8807 & 9330 & 800 & 3.4 & 1 & II: & $\mathrm{cD}:$ \\
2666 & $235101+270825$ & 8057 & 7714 & 8250 & 380 & $0.9^{*}$ & 0 & I & $\mathrm{cD}:$ \\
\hline \hline
\end{tabular}

* Temperature estimated from X-ray luminosity.

† Upper limit.

For purposes of reference in later sections, Table 4.2 contains information on the galaxy clusters themselves. For each cluster, the table lists J2000 coordinates of the centroid of the extended X-ray emission (Jones \& Forman 1996); mean velocities in the heliocentric, cosmic microwave background (CMB), and "Abell cluster inertial" (ACI) frames $\left(\mathrm{km} \mathrm{s}^{-1}\right.$; from PL and Postman 1996); velocity dispersion of the member galaxies $\left(\mathrm{km} \mathrm{s}^{-1}\right.$; described in §5.2.3); X-ray gas temperature ( $\mathrm{keV}$; from Jones \& Forman 1996); richness class and Bautz-Morgan type (Abell et al. 1989); and Rood-Sastry type (Struble \& Rood 1987). The ACI frame is the frame in which LP determined their clusters to have no net peculiar velocity according to the $L_{m}-\alpha$ distance indicator. The only cluster in the sample with a colloquial name is Abell 1656, the Coma cluster. 


\subsection{Observations}

\subsubsection{General Procedure}

The data used in this study were all obtained with the $2.4 \mathrm{~m}$ telescope at the MichiganDartmouth-MIT (MDM) Observatory on Kitt Peak over the course of several observing runs. Each night of observing, approximately 10 twilight flat fields were taken through the filter which was to be used for the deep imaging (either $R$ or $I$ ). Usually flat fields were needed in several bands, and oftentimes separate sets of flats were taken at both evening and morning twilight. The best flat field exposures were 4-10 seconds in duration, with the telescope pointed to the east (west) in the evening (morning). Shorter exposure flats suffered from shutter gradients, which sometimes had to be modeled and divided out due to their severity. Longer exposure flats were plagued by the presence of stars, which always needed to be removed.

The integration times for the individual exposures on the program galaxies ranged from 10 to 15 minutes so as to avoid saturation of stars in the fields which might later serve as templates for the image point spread function (PSF). Between exposures, the telescope was shifted by $5-10^{\prime \prime}$ in order to improve the image flattening, allow for the removal of cosmic ray hits and CCD defects, and provide a check on whether a series of exposures was truly photometric. The total integration times on the program galaxies ranged from just over $1 \mathrm{hr}$ to nearly $6.5 \mathrm{hr}$, determined by both the prevailing image quality and the galaxy distance, as detailed in $\S 4.3 .3$. If it was clear, about 10 images of Landolt (1992) photometric standard star fields were taken at varying airmasses throughout the night in each band for which photometry was needed. Standard star reductions and photometric calibration of the final galaxy images are discussed in $\S$ 4.3.2. The following subsection provides details on individual observing runs.

\subsubsection{Individual Runs}

Table 4.3 summarizes the information on all the observing runs which contributed data to this project. The runs are designated by the month and year in which they took place. For each run, the table lists the CCD camera that was used, the pixel 
Table 4.3: Observing Runs

\begin{tabular}{cccccccl}
\hline \hline Run & Detector & "/pix & $\mathrm{F}$ & $m_{1}$ & $A$ & $C$ & Notes \\
\hline 0593 & Loral 2048 & 0.343 & $I$ & 24.349 & 0.140 & 0.030 & Coma; $2 \times 2$ \\
0794 & STIS 2048 $^{2}$ & 0.240 & $I$ & 24.034 & 0.085 & 0.020 & N6166 \\
1194 & Tek 1024 & 0.275 & $R$ & 25.582 & 0.098 & 0.011 & poor weather \\
0295 & Tek 1024 & 0.275 & $R$ & 25.565 & 0.111 & 0.015 & \\
0395 & Tek 1024 & 0.275 & $R$ & 25.530 & 0.115 & 0.014 & much moon \\
0995 & Tek 1024 & 0.275 & $R$ & 25.340 & 0.117 & 0.015 & \\
\hline \hline
\end{tabular}

scale of the images in arcseconds per pixel, the filter through which the GC data were taken, the typical photometric coefficients $\left(m_{1}, A\right.$, and $C$, defined below in equation 4.1) used in calibrating the GC data, and some notes.

Collecting GC data on BCGs was not the primary goal of either of the first two runs. In the 0593 run, only the two Coma galaxies described in Chapter 2 were observed, while just one sample galaxy was observed during 0794 . I was the primary observer in 0593 and the lone observer in the last four runs. Mark Metzger was the observer in 0794 , and he generously obtained a deep image of NGC 6166, the BCG in A2199, for me as a filler project. The 0593 images were binned $2 \times 2$ to yield 0 ".343 $\mathrm{pix}^{-1}$; the 0794 data were significantly windowed due to vignetting. Due to its high quantum efficiency, the Tek chip was the detector of choice once it came online.

After an initial flirtation with the $I$ band (runs 0593 and 0794), I chose to take the remainder of the data in $R$ for several reasons:

1. The $R$ band is near the peak sensitivity of the CCD.

2. Thinned CCD chips such as the MDM Tek $1024^{2}$ show significant fringing in $I$.

3. The stellar SBF is relatively much stronger in $I$, with $\bar{m}_{R}-\bar{m}_{I} \sim 1.6$, while $(R-I) \sim 0.5$ for GCs.

4. The sky is much brighter in $I$.

5. PL report precise aperture photometry in $R$, which I had planned to used as a cross-check in the event of uncertain photometry.

A glass $R$ filter provided a much better match to standard $R$ bandpass, so it was 
used in lieu of an interference filter; the reverse was true in the $I$ band, where glass filters are very poor choice for CCD photometry. (Landolt used all glass filters, but his photomultiplier tube cuts off very sharply in the $I$ band at $9000 \AA$, unlike CCDs.) Further information on the data characteristics is given in $\S$ 4.3.3.

\subsection{Image Reductions}

\subsubsection{Initial Processing}

The initial data reduction steps for this Abell Cluster BCG sample are similar to those described in Chapter 2 (Blakeslee \& Tonry 1995) for the Coma cluster "pilot project." Here, I fill in some of the details and note any changes.

High signal-to-noise flat fields were produced for each night in the following manner. First, the 10-15 individual frames were bias subtracted using the overscan region of the CCD. The MDM Tek $1024^{2}$ has variable top-to-bottom structure in its bias; the structure was fitted using a $\sim 5$ th order spline and subtracted. The bias-subtracted frames were then windowed down to include only the data pixels and added together without visual inspection. Next, each frame was divided by this first-pass flat and inspected closely. Any stars were removed by masking to zero, and large scale gradients in the individual frames due to either a shifting illumination pattern from clouds present at twilight or (in $\lesssim 3 \mathrm{sec}$ exposures) shutter effects were fitted by a spline and divided out. Some frames were so badly affected that they were discarded.

Next, a program called "autoclean" (Tonry et al. 1997) was run on the series of flat field exposures. Autoclean identifies cosmic rays in a stack of images and removes them by replacing the affected pixels with appropriately scaled data values determined from the corresponding pixels' values in the rest of the stack. For flat fields, the scaling used for the replacement is done with respect to the means of the individual frames. The "star holes" in the images are filled in the same way. Failing to remove any significant large scale gradients from the individual frames can result in

poorly scaled replacement pixel values. After replacing cosmic rays and the masked stars, autoclean sums the frames to produce the final "cleaned" flat field. 
Table 4.4: Landolt Photometric Standard Fields

\begin{tabular}{|c|c|c|c|c|c|c|}
\hline Field & R.A. (J & 2000) Dec. & $V_{\min }$ & $V_{\max }$ & $(V-I)_{\min }$ & $(V-I)_{\max }$ \\
\hline SA92-250 & 005441 & +004111 & 14.09 & 15.35 & 0.67 & 1.34 \\
\hline SA95-190 & 035316 & +001625 & 12.63 & 14.34 & 0.42 & 1.37 \\
\hline SA95-275 & 035440 & +002724 & 12.17 & 14.12 & 1.40 & 2.27 \\
\hline SA98-650 & 065211 & -001923 & 11.93 & 13.75 & 0.17 & 2.09 \\
\hline Rubin-149 & 072413 & -003158 & 11.48 & 13.87 & -0.11 & 1.13 \\
\hline PG0918+029 & 092136 & +024703 & 12.27 & 14.49 & -0.29 & 1.11 \\
\hline PG1323-085 & 132544 & $-0849 \quad 16$ & 12.08 & 14.00 & -0.13 & 0.83 \\
\hline PG1633+099 & 163529 & +094654 & 12.97 & 15.27 & -0.21 & 1.14 \\
\hline SA110-232 & 184050 & +000151 & 12.52 & 14.28 & 0.89 & 2.36 \\
\hline SA110-503 & 184305 & +002910 & 11.31 & 14.20 & 0.65 & 2.63 \\
\hline Markarian A & 204359 & -104742 & 13.26 & 14.82 & -0.24 & 1.10 \\
\hline
\end{tabular}

The galaxy data and standard star calibration frames were bias subtracted and windowed in the same manner as the flats. They are then divided by the night's cleaned and normalized flat field. The next two sections describe subsequent reductions of the calibration and data images.

\subsubsection{Photometric Calibration}

Table 4.4 lists the standard star fields used for this project. They were selected primarily because of the large number of observations made by Landolt on each, and consequent small magnitude uncertainties, and because of the number of stars present within $\mathrm{a} \sim 5^{\prime} \times 5^{\prime}$ field.

The standard star observations were used to determine the photometric coefficients for each clear night as follows. Following Landolt (1992), and as in Tonry et al. (1997), the net flux from each photometric standard within a $14^{\prime \prime}$ aperture was summed. Standard star measurements with estimated errors larger than 0.02 mag from flux, sky, and Landolt magnitude uncertainties were discarded. After all the standard star observations from a run were reduced, the results were fitted according to

$$
m=-2.5 \log (f / t)-A \sec (z)+C(V-I)+m_{1}
$$



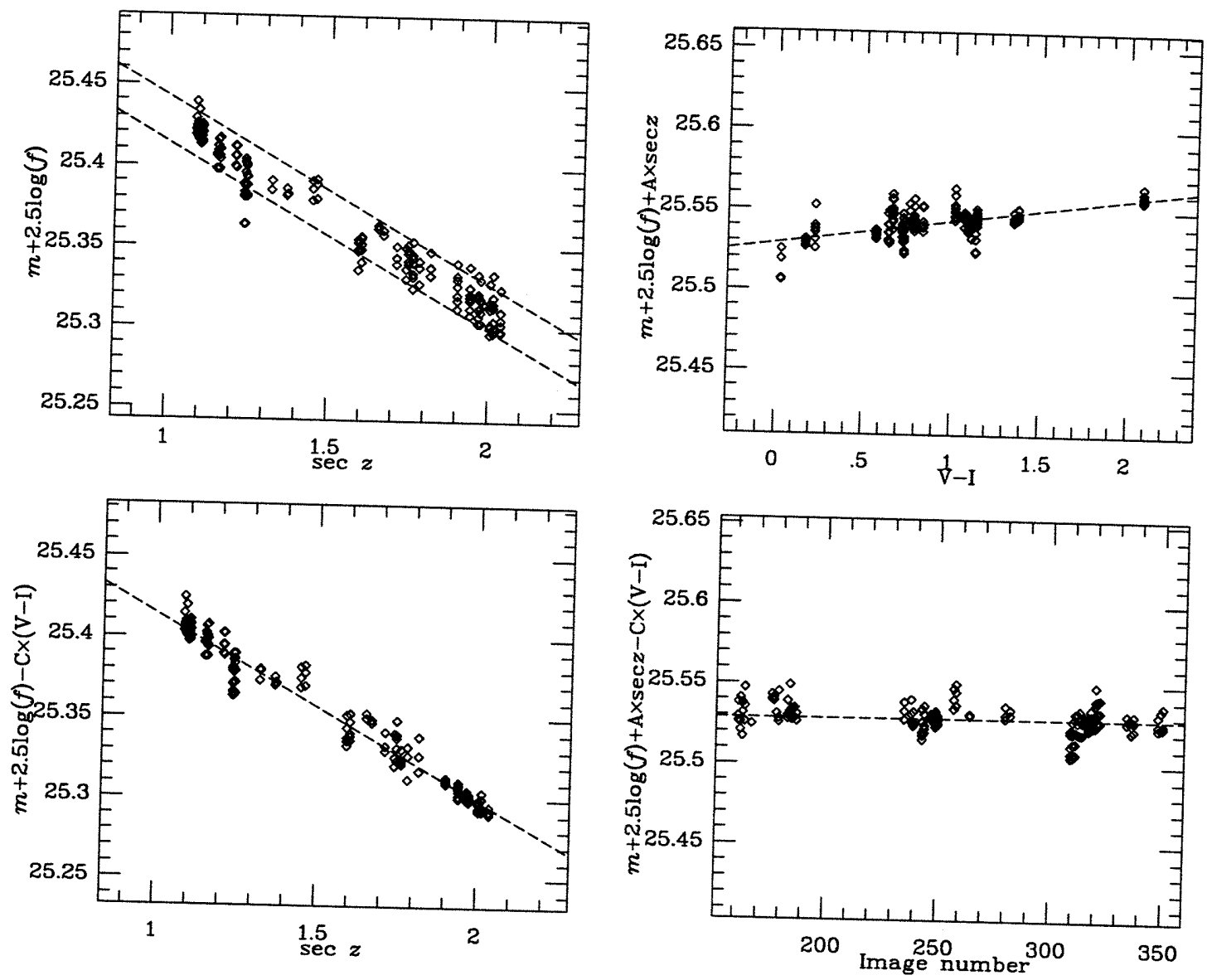

Figure 4-1: The results of the $R$-band photometric standard star reductions for the 0395 run. Three nights are represented, but a single set of coefficients was used for all. Upper left panel shows the raw photometry as a function of the airmass, $\sec z$; upper right shows the color dependence after the $\sec z$ dependence has been removed; lower left shows the $\sec z$ dependence after removal of the color dependence; lower right shows final residuals plotted against image number after removing both color and $\sec z$ dependences. The rms scatter in the residuals is 0.008 mag.

where $m$ is the magnitude reported by Landolt in the appropriate band, $f$ the total counts in electrons from the star within $14^{\prime \prime}, t$ the exposure time in seconds, $z$ the zenith angle, and $(V-I)$ comes from Landolt. Typical values of $A, C$, and $m_{1}$ were given in Table 4.3 for each run. On truly clear nights, the scatter about the derived relation was $\lesssim 0.01 \mathrm{mag}$. As an example, Figure 4-1 shows the results of the standard star reductions and photometric coefficient fits for a single run. 


\subsubsection{The Galaxy Images}

As in the previous chapters, the individual exposures which make up a galaxy observation were bias subtracted and flattened before being brought into registration based on the positions of stars in the images. Bad pixels were masked and the frames were then combined to make the final image using autoclean. For this purpose, autoclean does the scaling to replace bad pixels by fitting each image as a linear function of the first one in the stack. Very large image stacks ( 20 frames), were broken into two, and autoclean was run on each stack. The two resulting images were then summed to make the final cleaned image. The following two subsections discuss the properties of the final images which make up the present data set.

\subsubsection{Image Quality}

After the initial $I$-band Coma observations in run 0593, I attempted in subsequent runs to integrate long enough on each galaxy to reach within $\sim 2$ mag of the expected GCLF turnover $m^{0}$ while the data were still $\gtrsim 90 \%$ complete. Due to the vicissitudes of weather and telescope scheduling, this goal was not always attained. However, as will be seen in Chapter 5, the data still proved more than adequate in most cases.

One measure of the depth of an observation is the quantity $m_{1}^{*}$, the magnitude of an object which will produce one count per total image integration time, corrected for Galactic extinction:

$$
m_{1}^{*}=2.5 \log (t)-A \sec (z)+C(V-I)+m_{1}-A_{\lambda} .
$$

where $A_{\lambda}$ is the extinction in the $\lambda$ band. Thus, as in Chapter 2, the extinction corrected magnitude of an object yielding $f$ total counts in the image can be calculated as: $m=-2.5 \log (f)+m_{1}^{*}$. So, $m_{1}^{*}$ indicates the amount of signal in an image, but takes no account of the noise.

It would be helpful to have some measure of the quality of an image, i.e., the suitability of an observation for yielding the desired information. We start by considering the signal-to-noise ratio (SNR) of a sky background limited object which produces $f$ 
counts within the point spread function width:

$$
\mathrm{SNR}=\frac{f}{\sqrt{b \times \mathrm{PSF}^{2}}}
$$

where $b$ is the sky level in counts/arcsec ${ }^{2}$ and PSF is the FWHM in arcsec. To calculate the true signal-to-noise, the galaxy surface brightness would need to be taken into account, but this is useful for comparing images with similar galaxy surface brightnesses. It is interesting to view this equation in terms of magnitudes by taking the logarithm and simplifying:

$$
2.5 \log (\mathrm{SNR})=-2.5 \log (\mathrm{PSF})-m_{\mathrm{FW}}+0.5\left(m_{1}^{*}+\mu_{s k y}\right)
$$

where $m_{\mathrm{FW}}$ is the magnitude of the object within the PSF FWHM and $\mu_{s k y}$ is the sky surface brightness in mag/arcsec ${ }^{2}$. For a PSF which approximates a two dimensional Gaussian, the total flux is about twice the flux within the FWHM. The obvious characteristic magnitude for GC studies is $m^{0}$, the point at which the GCLF turns over. The limiting magnitudes of the images in this project do not generally approach $m^{0}$; however, if we plug in $m_{\mathrm{FW}}=m^{0}$, the object under consideration will be one which is twice as bright as $m^{0}$, e.g., a globular cluster $0.75 \mathrm{mag}$ brighter the GCLF turnover. Equation 4.4 then becomes:

$$
2.5 \log \left(\mathrm{SNR}^{0}\right)=-2.5 \log (\mathrm{PSF})+0.5\left(m_{1}^{*}+\mu_{s k y}\right)-m^{0}
$$

where $I$ have called this fiducial signal-to-noise ratio $\mathrm{SNR}^{0}$, although it actually refers to an object twice as bright as, not equal to, $m^{0}$.

The quantity calculated in equation 4.5 is an interesting one; in $1^{\prime \prime}$ seeing, it is positive if the average of $m_{1}^{*}$ and $\mu_{s k y}$ is fainter than $m^{0}$ for the galaxy, but perhaps more intuitive is $\mathrm{SNR}^{0}$ itself:

$$
\mathrm{SNR}^{0}=\mathrm{PSF}^{-1} \times 10^{0.2\left(m_{1}^{*}+\mu_{s k y}-2 m^{0}\right)}
$$


which provides a useful indicator of the data image quality once $m^{0}$ has been estimated. As in Chapters 2 and 3, this will be done relative to the measured value of $m^{0}$ in Virgo. Using the CMB velocity of Coma given in Table 4.2 and the relative Coma-Virgo distance modulus, we have

$$
m^{0}(z)=m_{\mathrm{Vir}}^{0}+5 \log \left(\frac{c z}{1310}\right)
$$

\subsubsection{Final Data Set}

Table 4.5 presents the data set used for this study of the GCSs of Abell cluster galaxies. For each galaxy image, the table lists the observing run in which the data were taken, the total exposure time in seconds, the seeing FWHM in arcsec, $m_{1}^{*}$ as defined in equation 4.2 , the sky brightness in $\mathrm{mag} / \mathrm{arcsec}^{2}, \mathrm{SNR}^{0}$ calculated as described above, and the total number of unique objects identified in the image down to a signal-to-noise limit of 4.0 by the photometry program DoPhot (Schechter, Mateo, \& Saha 1993; DoPhot and the point source photometry will be discussed in $\S$ 4.4.1). The table shows that this project benefitted from both a generous amount of observing time and some good seeing. Galaxies A347-1 and A569-1 were both observed twice, and each observation was reduced independently. For these two galaxies the strong dependence of the number of identified objects on $\mathrm{SNR}^{0}$ is clearly evident.

Of course, $\mathrm{SNR}^{0}$ does not tell the whole story. Total signal-to-noise scales also with the square root of the number of GCs present in the image. This cannot be known ahead of time, though some estimates might be made based on galaxy luminosity. For comparison purposes, Table 4.5 includes the information on the Coma observations presented and analyzed in Chapter 2. Both of these galaxies happened to have very rich GC populations. Consequently, it was possible to learn a good deal about them, despite the fact that their $\mathrm{SNR}^{0}$ values are among the lowest in the table. (Although the comparison is not really fair, since those images were in the $I$-band, where the sky is much brighter but the galaxy surface brightness is not, and as noted above, the galaxy brightness is not taken into account here.) 
Table 4.5: Galaxy Observations

\begin{tabular}{rrrrcccc}
\hline \hline Galaxy & Run & Exp & PSF & $m_{1}^{*}$ & $\mu_{\text {sky }}$ & SNR $^{0}$ & $\mathrm{~N}_{\text {obj }}$ \\
\hline $\mathrm{A} 262-1$ & 1194 & 5600 & 1.10 & 34.681 & 20.75 & 4.4 & 1394 \\
$\mathrm{~A} 347-1$ & 1194 & 4000 & 0.99 & 34.348 & 20.67 & 3.0 & 1320 \\
$\mathrm{~A} 347-1$ & 0995 & 4200 & 0.86 & 34.145 & 21.05 & 3.8 & 2351 \\
$\mathrm{~A} 397-1$ & 0995 & 20700 & 0.87 & 35.852 & 20.29 & 1.8 & 1350 \\
$\mathrm{~A} 539-1$ & 0295 & 11900 & 0.93 & 35.348 & 20.92 & 2.2 & 1300 \\
$\mathrm{~A} 539-2$ & 0295 & 10850 & 0.89 & 35.250 & 20.77 & 2.0 & 1511 \\
$\mathrm{~A} 569-1$ & 0295 & 4200 & 0.96 & 34.335 & 21.18 & 3.3 & 1210 \\
$\mathrm{~A} 569-1$ & 0395 & 6000 & 1.24 & 34.656 & 20.96 & 2.7 & 867 \\
$\mathrm{~A} 634-1$ & 0295 & 12600 & 0.98 & 35.626 & 21.24 & 3.1 & 1796 \\
$\mathrm{~A} 779-1$ & 0295 & 6600 & 0.90 & 35.018 & 21.14 & 3.3 & 2086 \\
$\mathrm{~A} 999-1$ & 0295 & 13200 & 0.88 & 35.679 & 21.12 & 2.3 & 1802 \\
$\mathrm{~A} 1016-1$ & 0295 & 11700 & 0.86 & 35.597 & 21.09 & 2.2 & 1590 \\
$\mathrm{~A} 1177-1$ & 0295 & 15025 & 0.96 & 35.903 & 21.14 & 2.4 & 2157 \\
$\mathrm{~A} 1185-1$ & 0395 & 23100 & 0.91 & 36.310 & 19.78 & 1.5 & 1423 \\
$\mathrm{~A} 1314-1$ & 0395 & 19500 & 1.03 & 36.120 & 21.21 & 2.4 & 2222 \\
$\mathrm{~A} 1367-1$ & 0295 & 4200 & 0.77 & 34.501 & 20.77 & 2.7 & 2113 \\
$\mathrm{~A} 1656-1$ & 0593 & 4900 & 0.88 & 33.392 & 19.02 & 0.9 & 1216 \\
$\mathrm{~A} 1656-2$ & 0593 & 5500 & 0.83 & 33.542 & 19.10 & 1.1 & 1663 \\
$\mathrm{~A} 1656-3$ & 0395 & 10800 & 1.00 & 35.479 & 19.95 & 2.0 & 1429 \\
$\mathrm{~A} 2162-1$ & 0995 & 18900 & 1.29 & 35.789 & 20.59 & 1.3 & 1196 \\
$\mathrm{~A} 2197-1$ & 0395 & 19300 & 0.99 & 36.127 & 20.03 & 1.8 & 1210 \\
$\mathrm{~A} 2197-2$ & 0395 & 12800 & 1.05 & 35.689 & 21.26 & 2.5 & 1786 \\
$\mathrm{~A} 2199-1$ & 0794 & 11700 & 0.92 & 34.135 & 19.82 & 1.1 & 2530 \\
$\mathrm{~A} 2634-1$ & 0995 & 13700 & 1.01 & 35.428 & 20.04 & 1.4 & 1455 \\
$\mathrm{~A} 2666-1$ & 1194 & 8200 & 0.91 & 35.023 & 20.83 & 2.4 & 1036 \\
\hline \hline
\end{tabular}




\subsubsection{A BCG Bestiary}

\subsubsection{Contour Plots}

Figures 4-2 through Figures 4-24 display the isophotal contour maps for the sample galaxies, generated from the images listed in Table 4.5. The contours are plotted in one-magnitude increments, with the outermost contour at $R=24.5 \mathrm{mag} / \mathrm{arcsec}^{2}$, corrected for Galactic extinction. For the $I$-band images of A2199-1 and the 0593 Coma galaxies, $(R-I)_{0}=0.64$ has been assumed in calculating $R$ isophotes. Each figure shows an area $4^{\prime} .5 \times 4^{\prime} .5$ in size. The coordinate axes have their origin at the center of each image, but this does not always precisely coincide with the galaxy center. For the $R$-band images, north is to the left and east at bottom; for the A2199-1 image, north is at top and east to the left; for A1656-1 and A1656-2, north is at top and east is to the right. Thus, as conventional, the R.A. and Dec. coordinates are shown increasing towards north and east, respectively.

The two $I$-band Coma images are shown here because no similar plots appeared in Blakeslee \& Tonry (1995). They were taken with the MDM Loral CCD which shows a gradient of $\sim 2 \%$ in the $I$-band night sky when flattened with twilight flats. Thus, the distortion in the outer isophote of A1656-1 is probably not real. The distortion is not obvious in A1656-2 because of its greater extension and more rounded isophotes. The $I$-band STIS data and $R$-band Tek data which comprise everything since that initial project show no significant sky gradients. Following the contour plots, further information is provided concerning individual galaxies in the sample. 


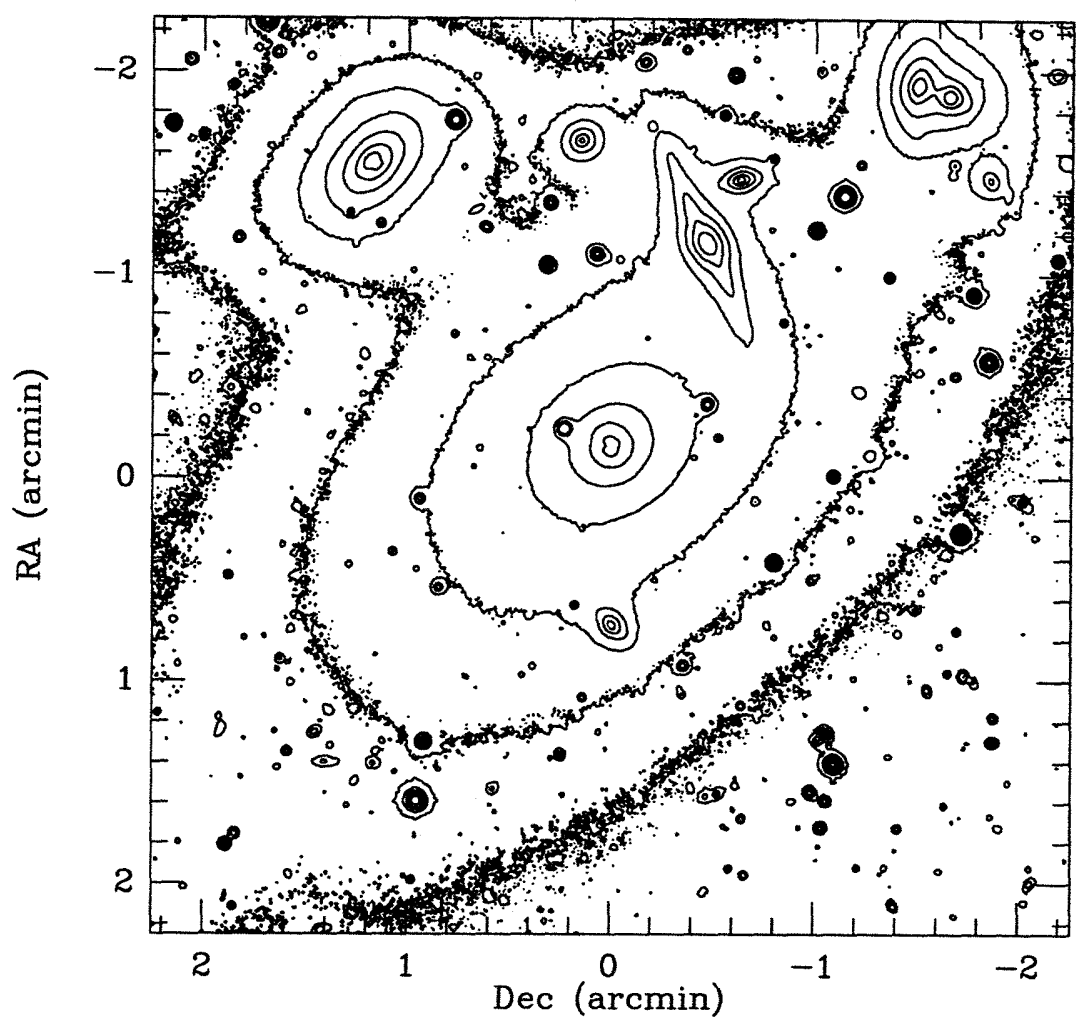

Figure 4-2: A262-1.

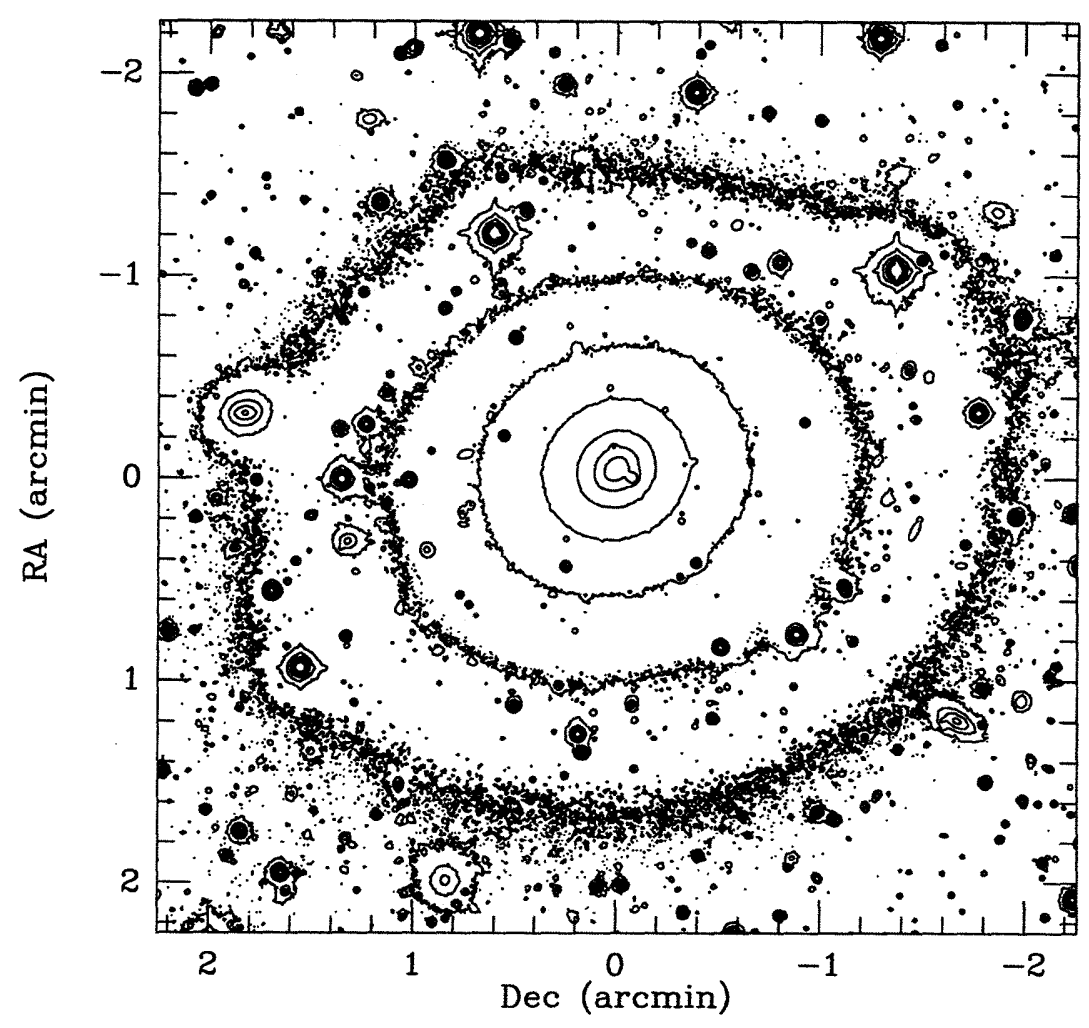

Figure 4-3: A347-1. 


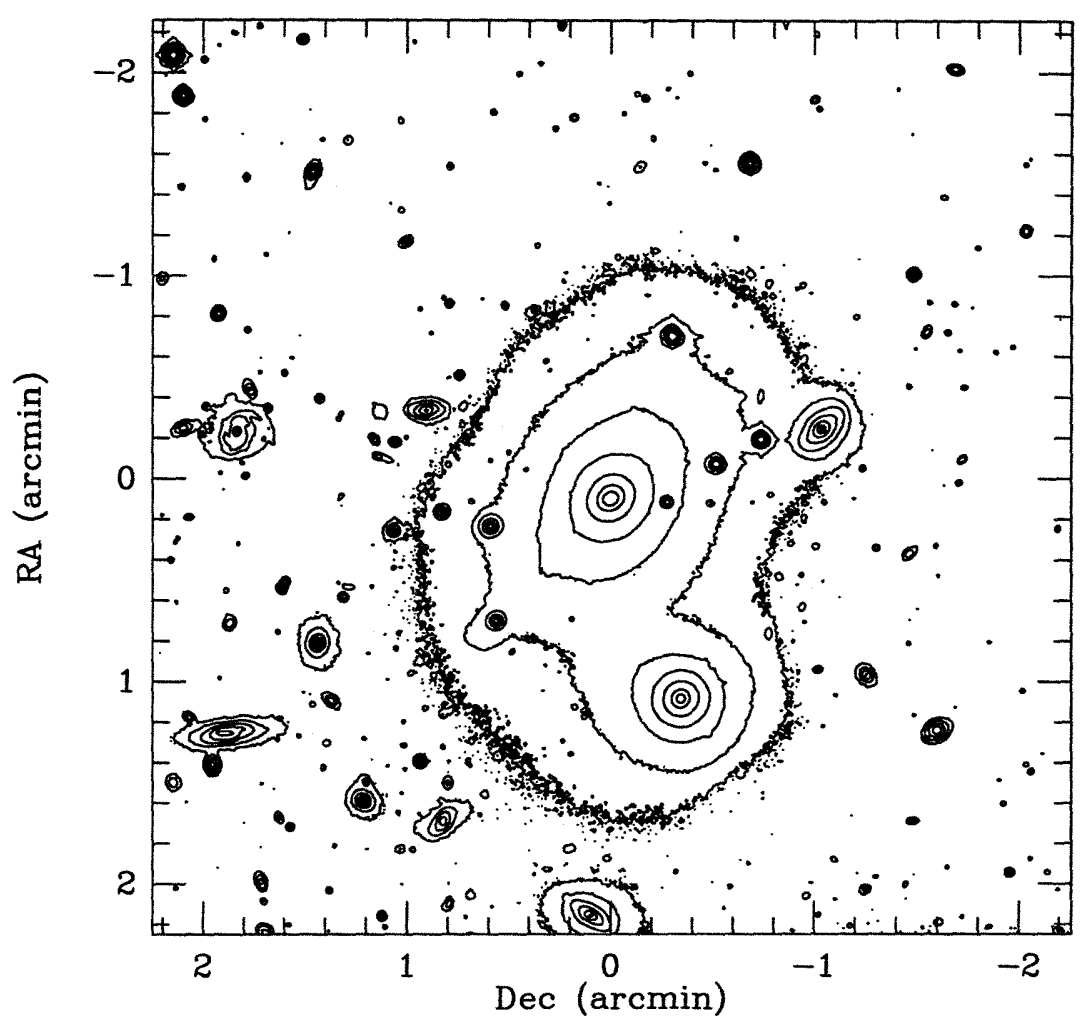

Figure 4-4: A397-1.

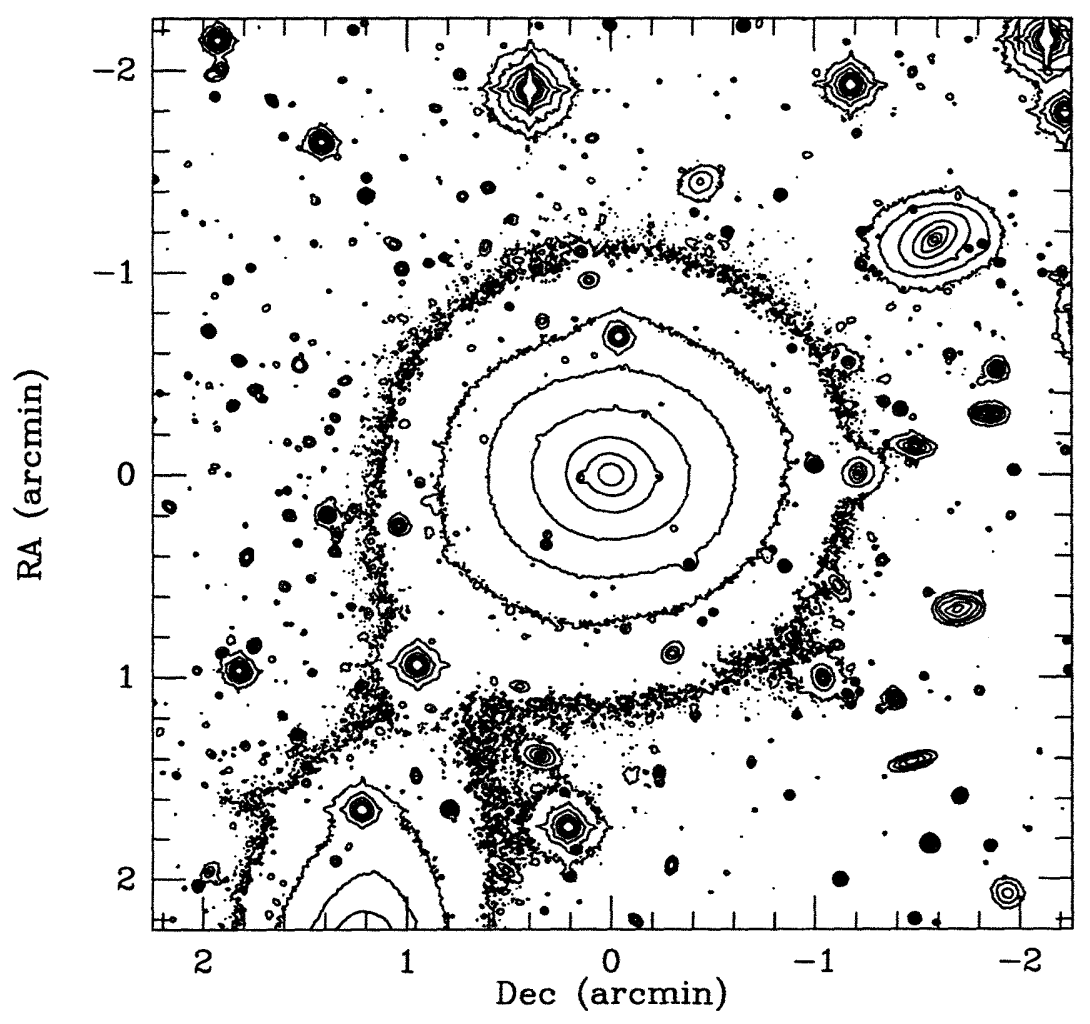

Figure 4-5: A569-1. 


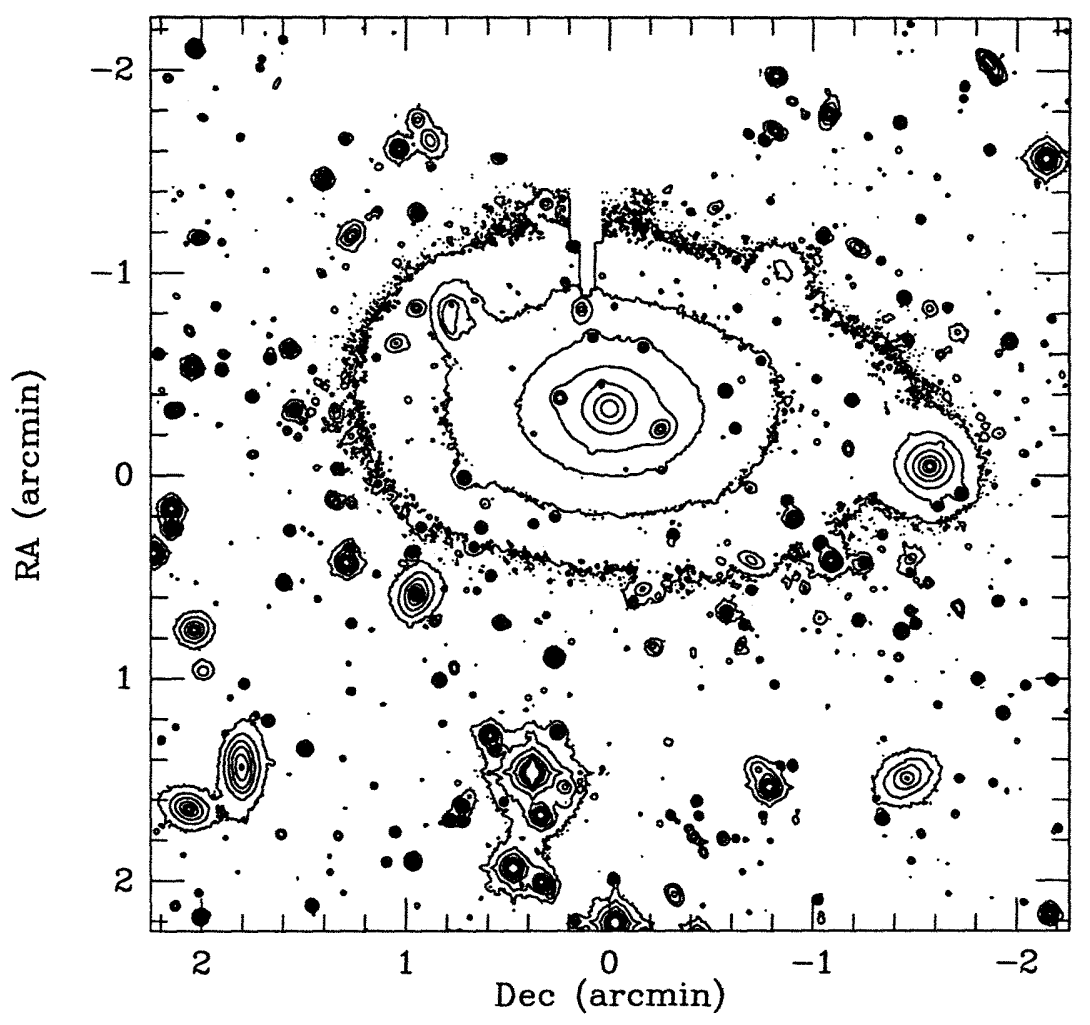

Figure 4-6: A539-1.

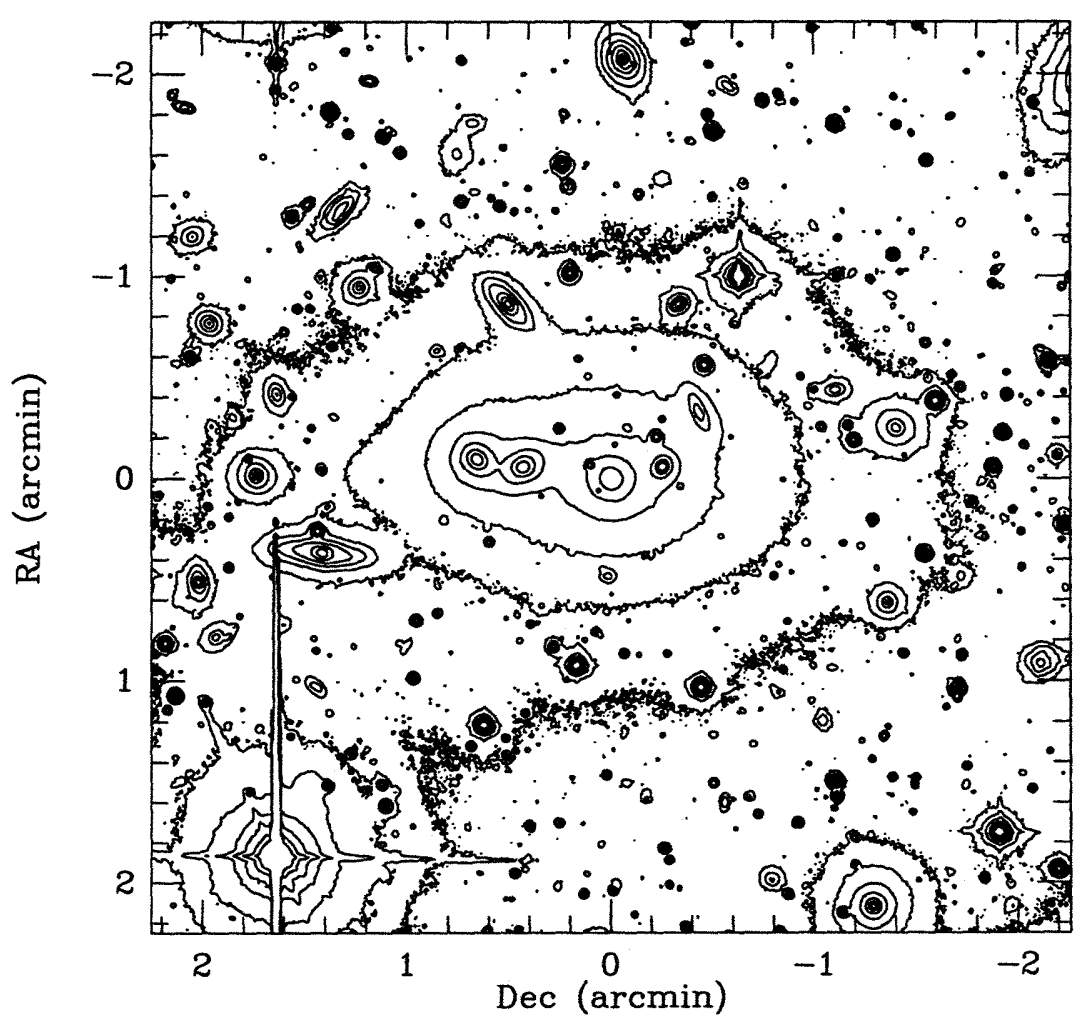

Figure 4-7: A539-2. 


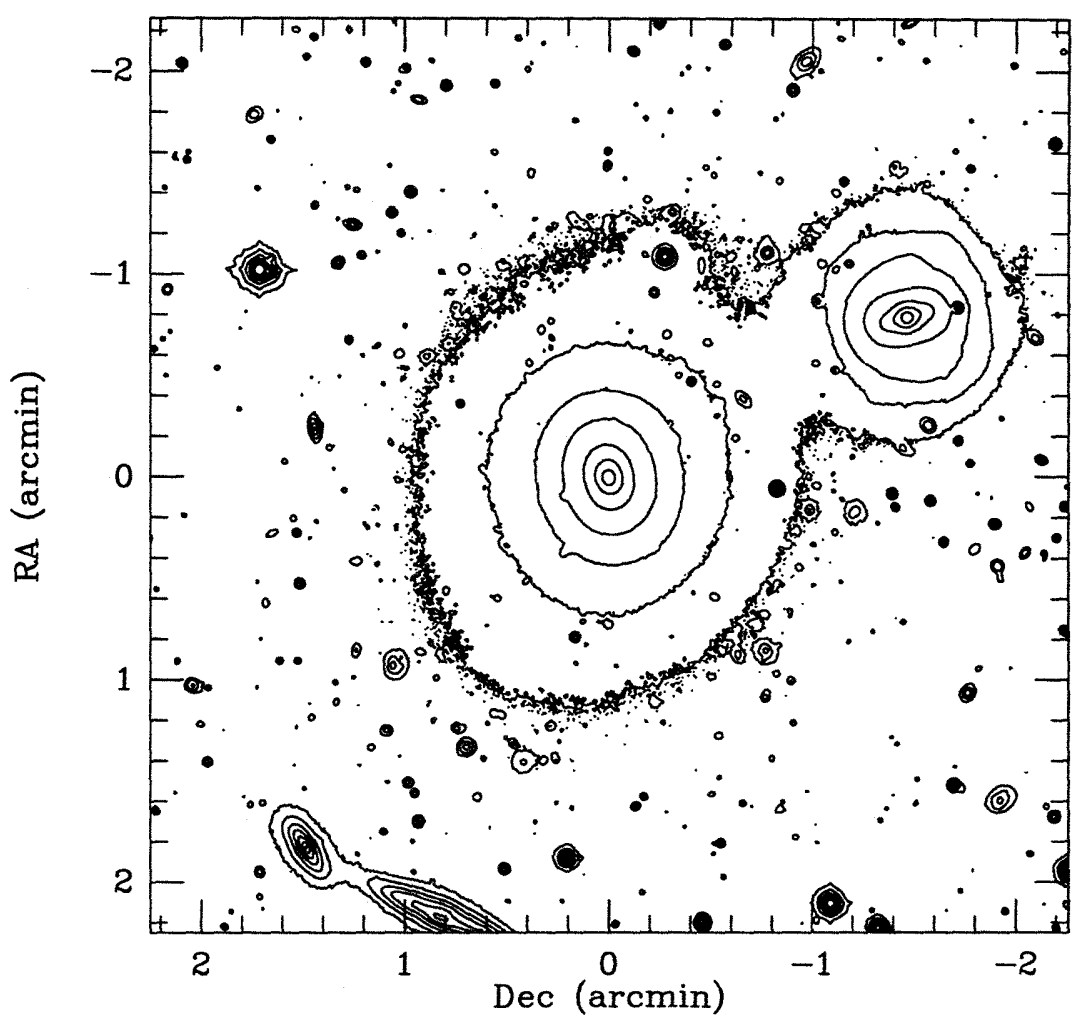

Figure 4-8: A634-1.

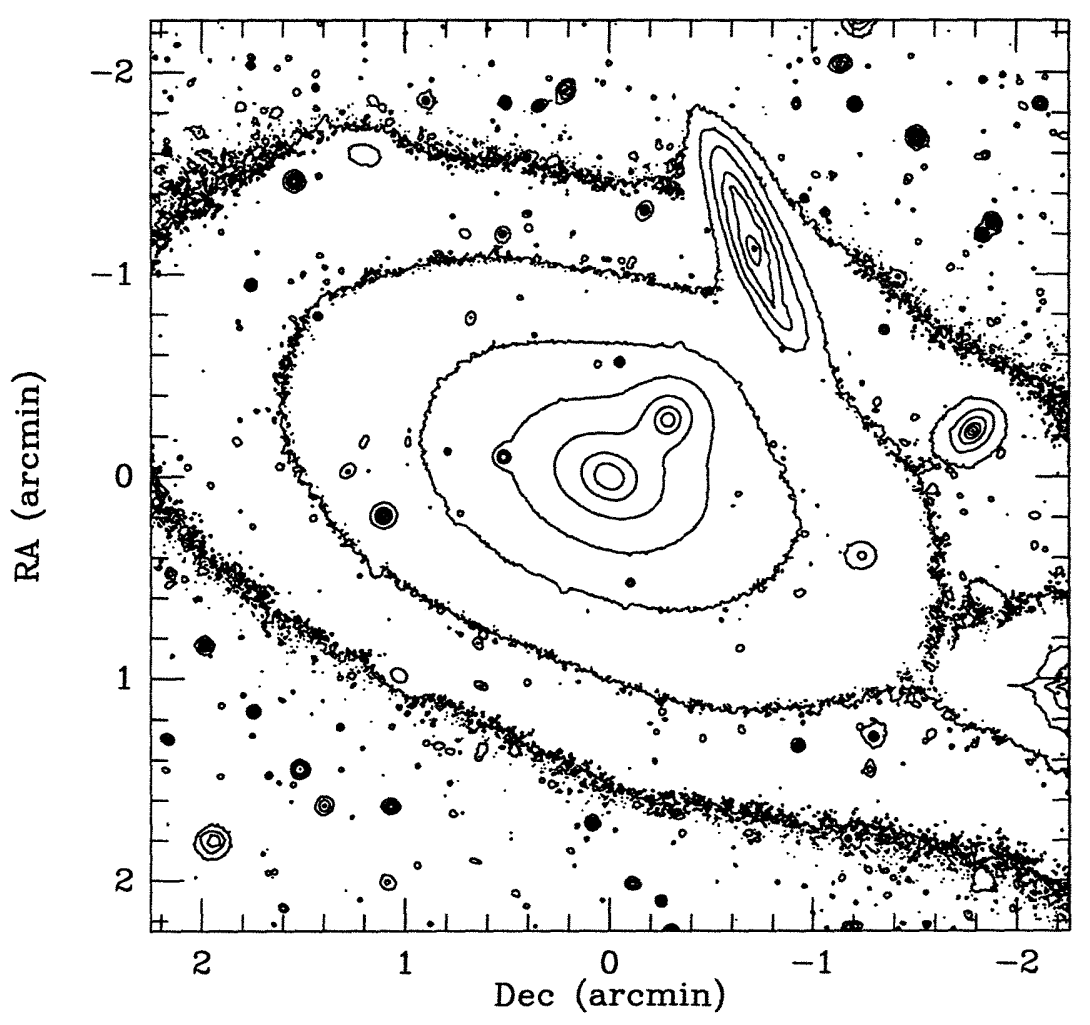

Figure 4-9: A779-1. 


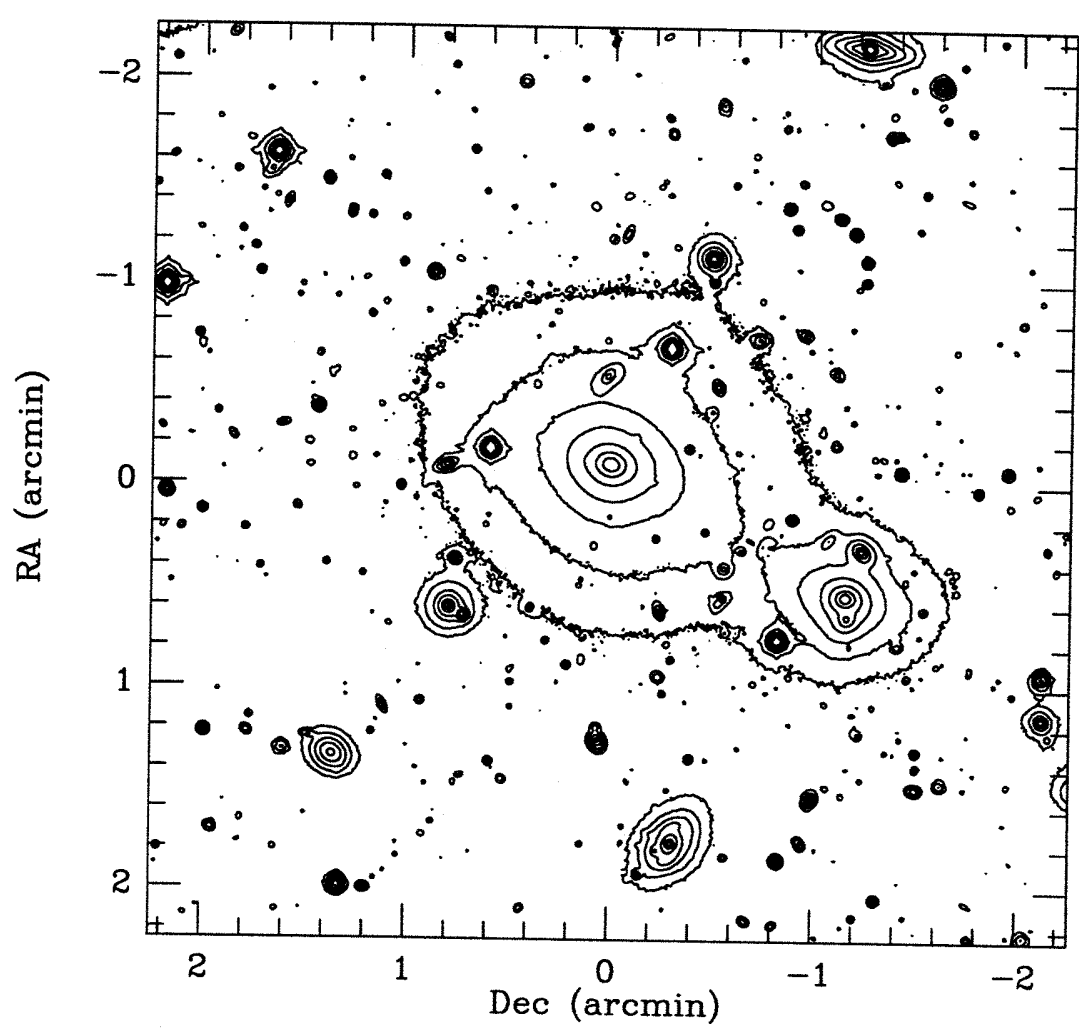

Figure 4-10: A999-1.

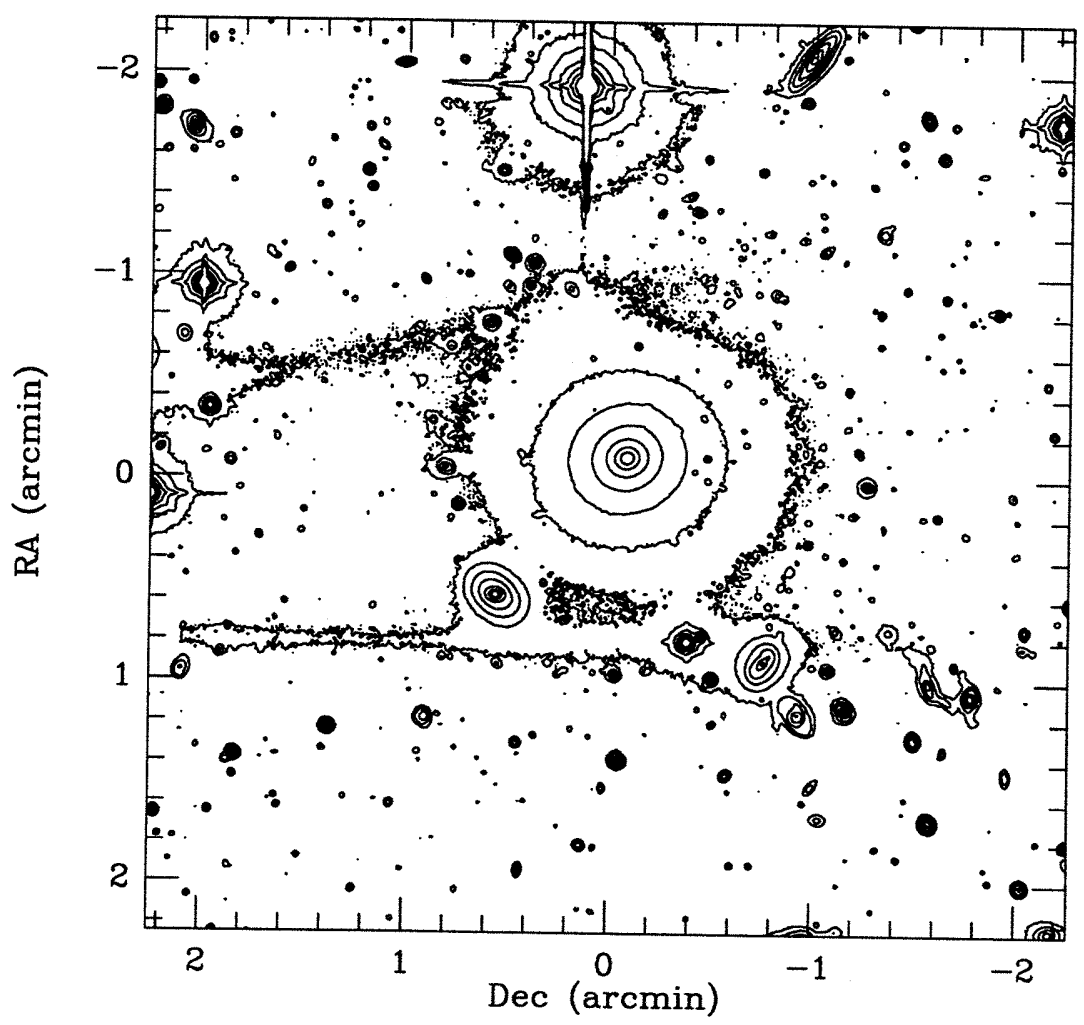

Figure 4-11: A1016-1. 


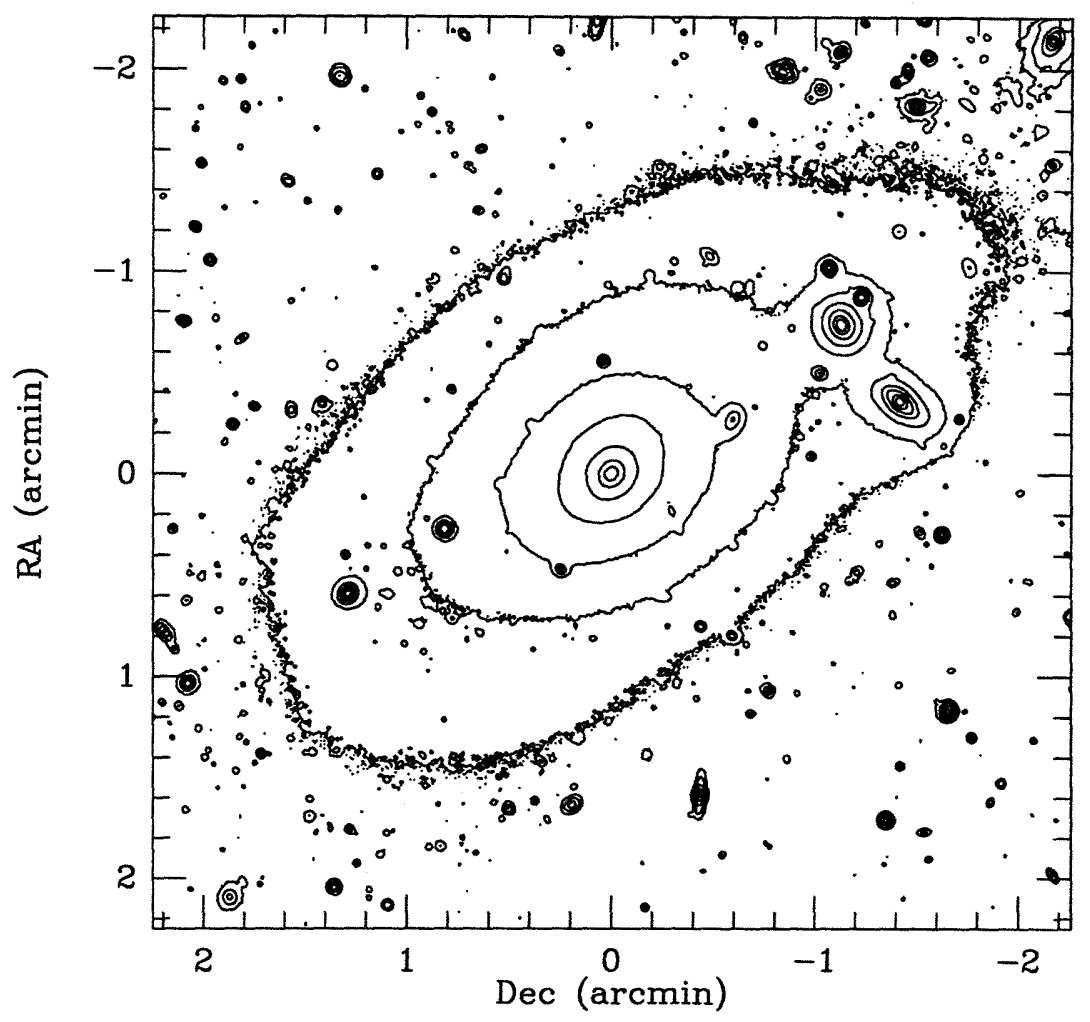

Figure 4-12: A1177-1.

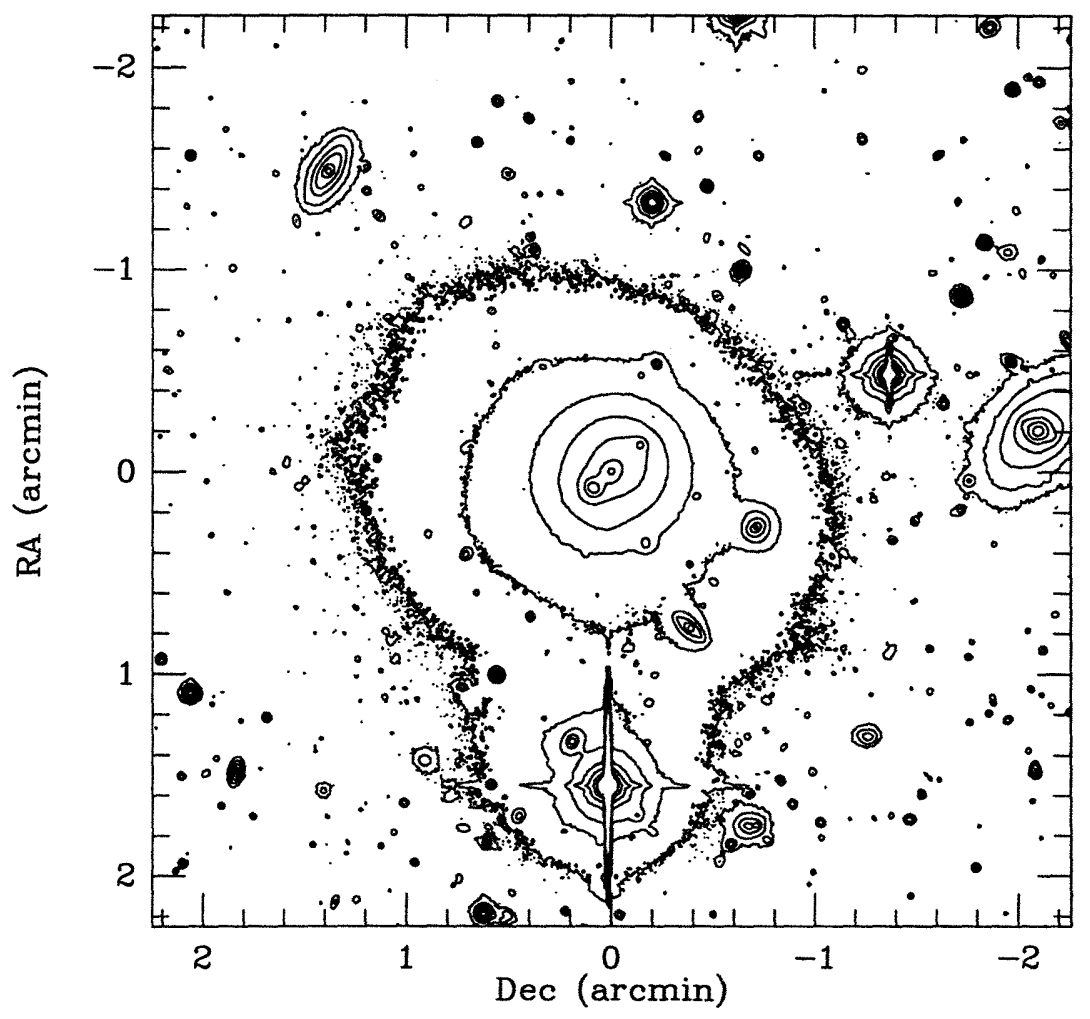

Figure 4-13: A1185-1. 


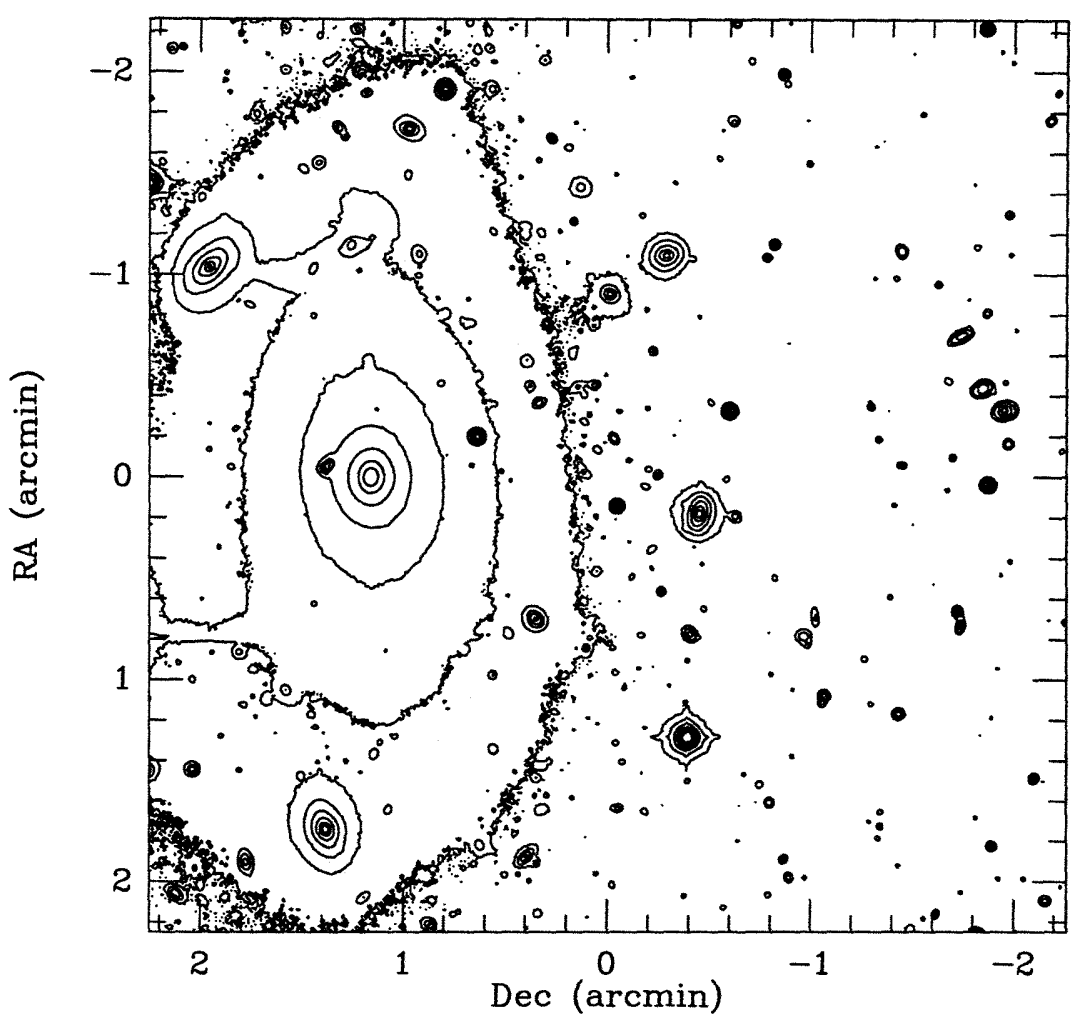

Figure 4-14: A1314-1.

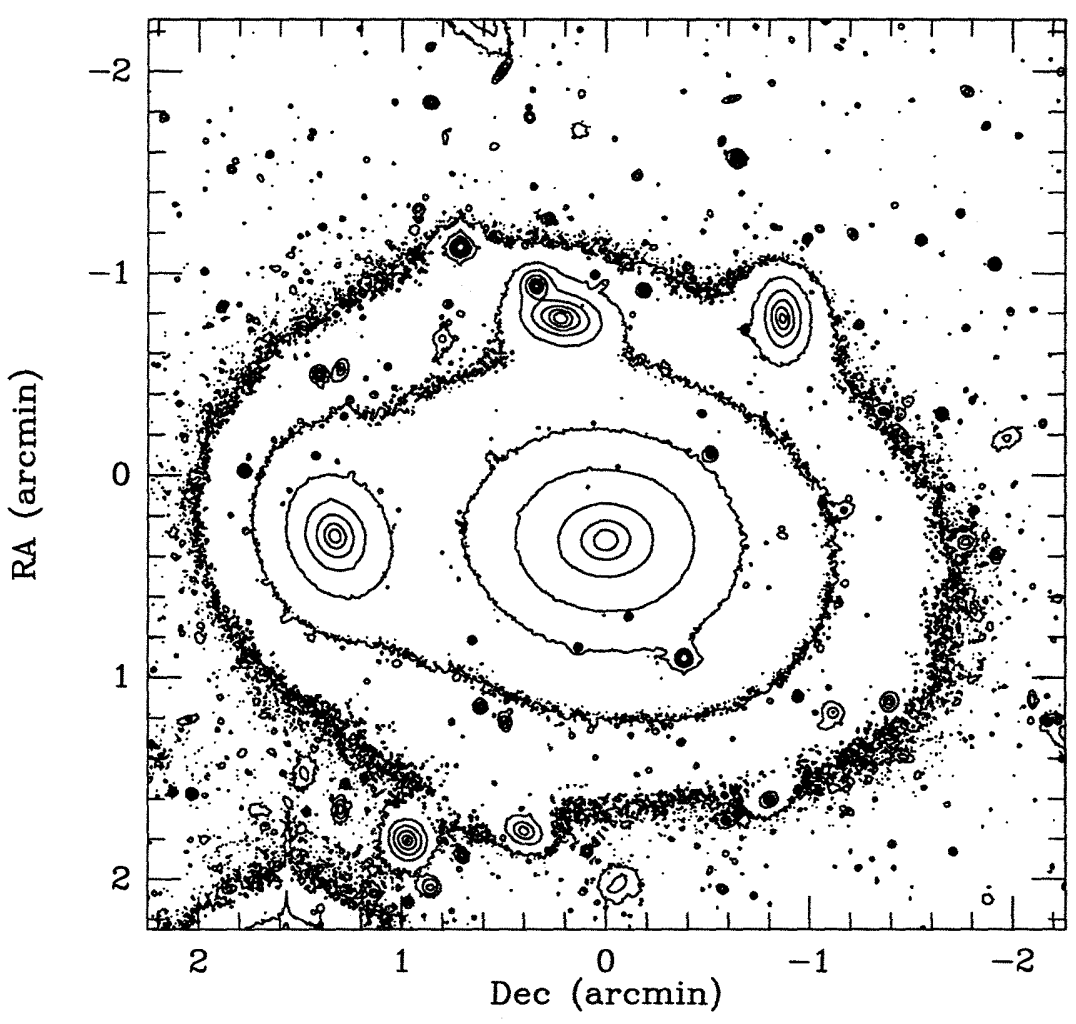

Figure 4-15: A1367-1. 


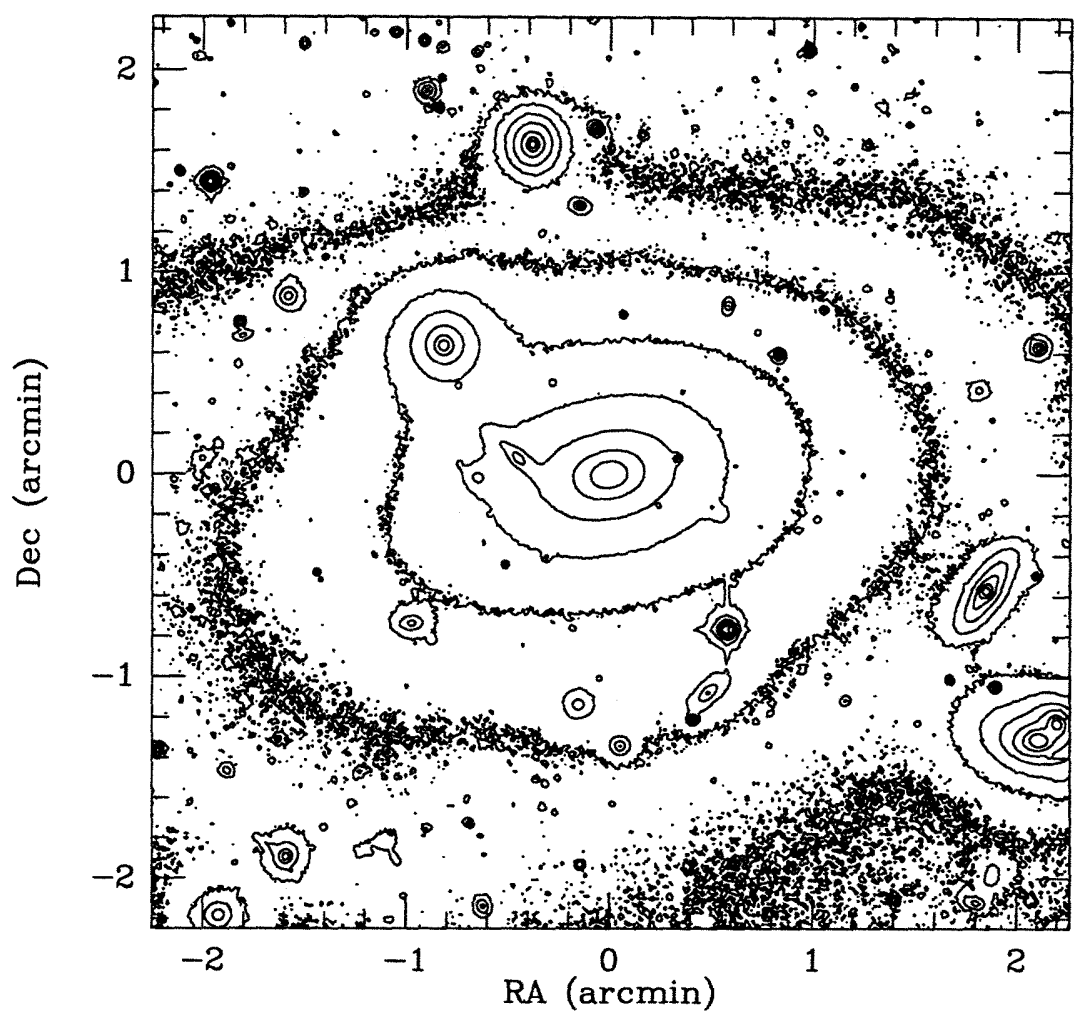

Figure 4-16: A1656-1.

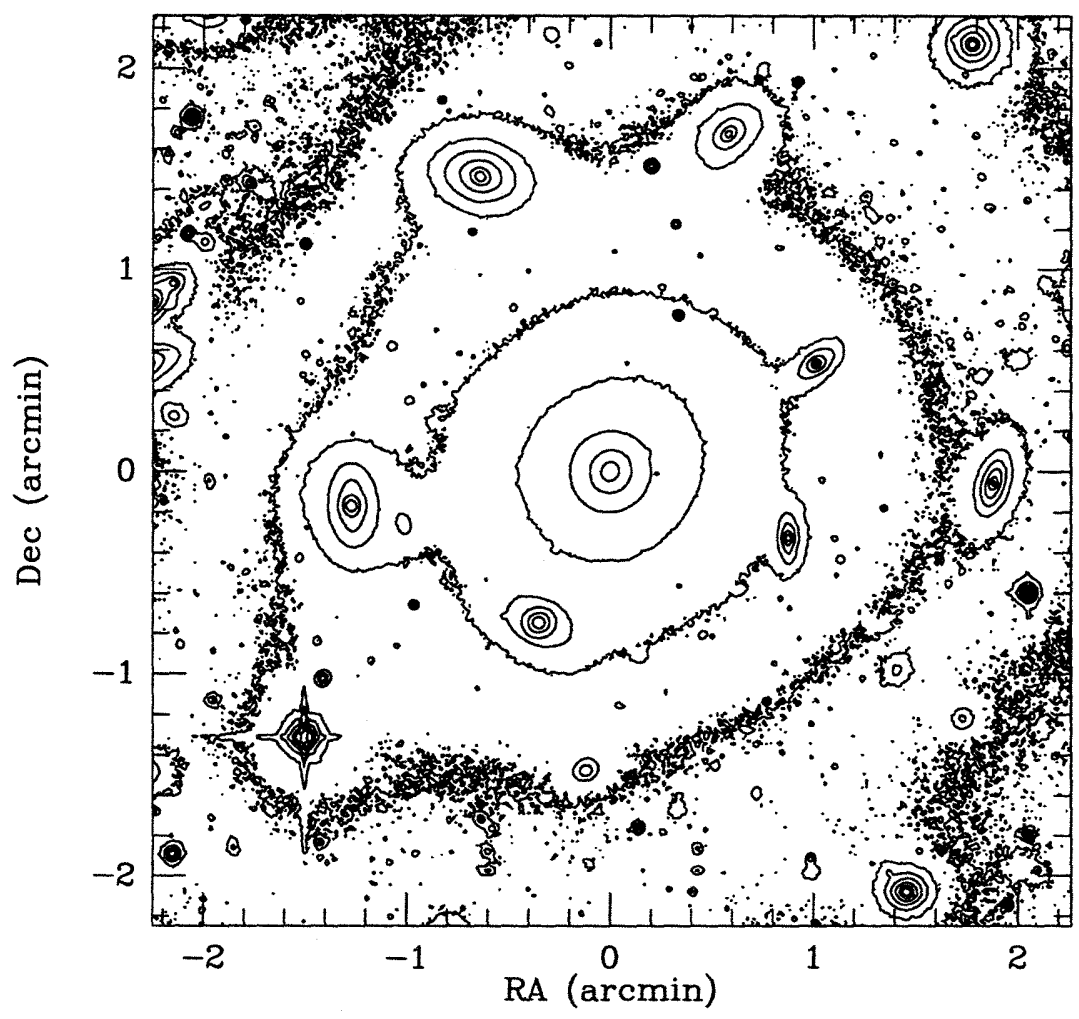

Figure 4-17: A1656-2. 


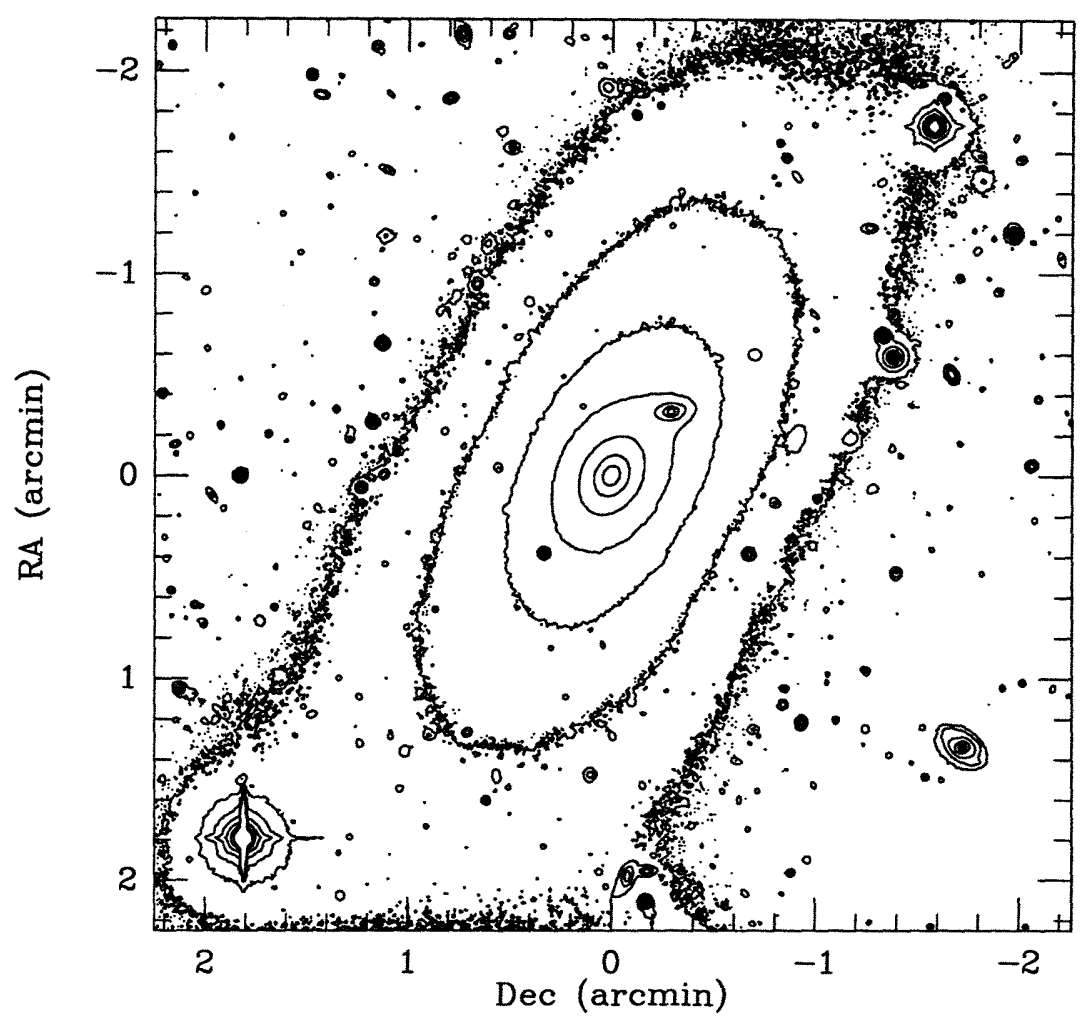

Figure 4-18: A1656-3.

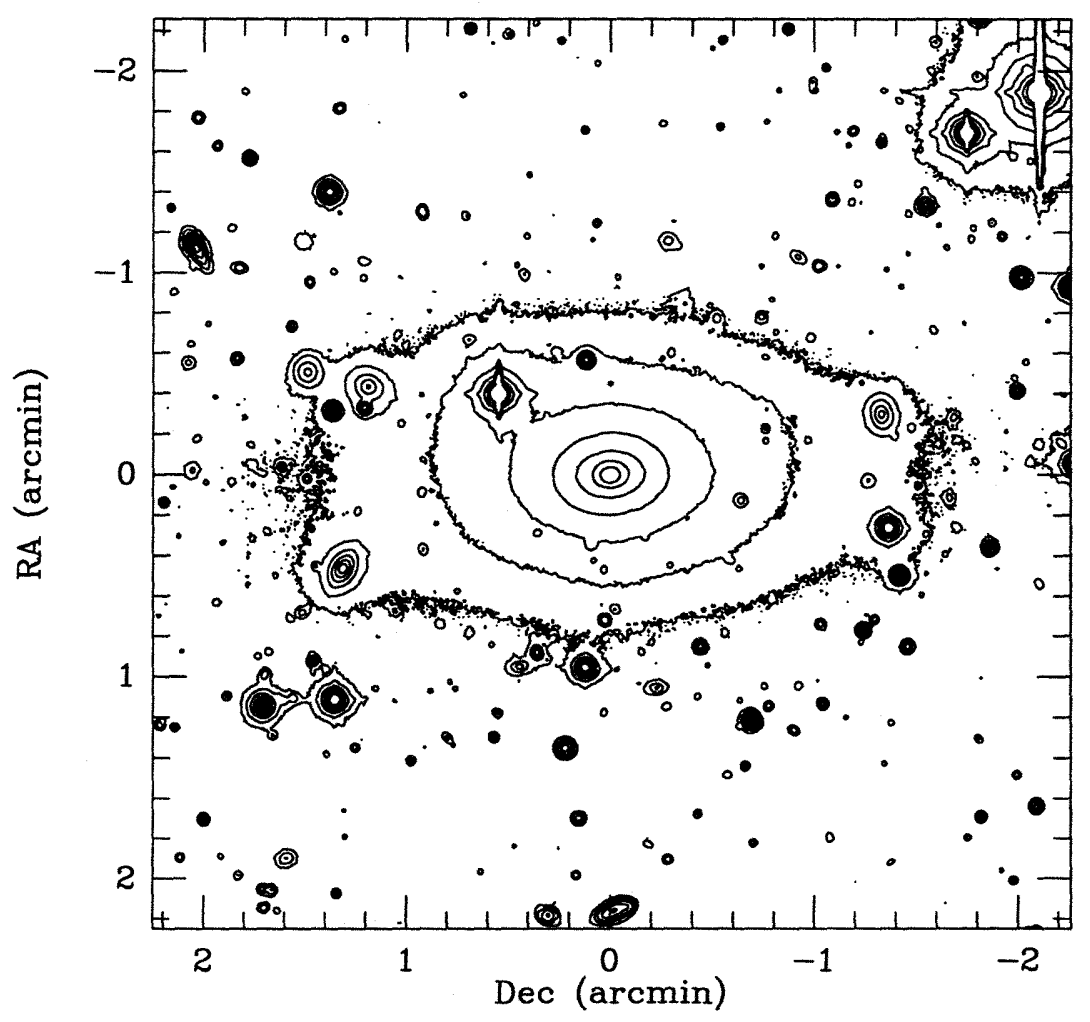

Figure 4-19: A2162-1. 


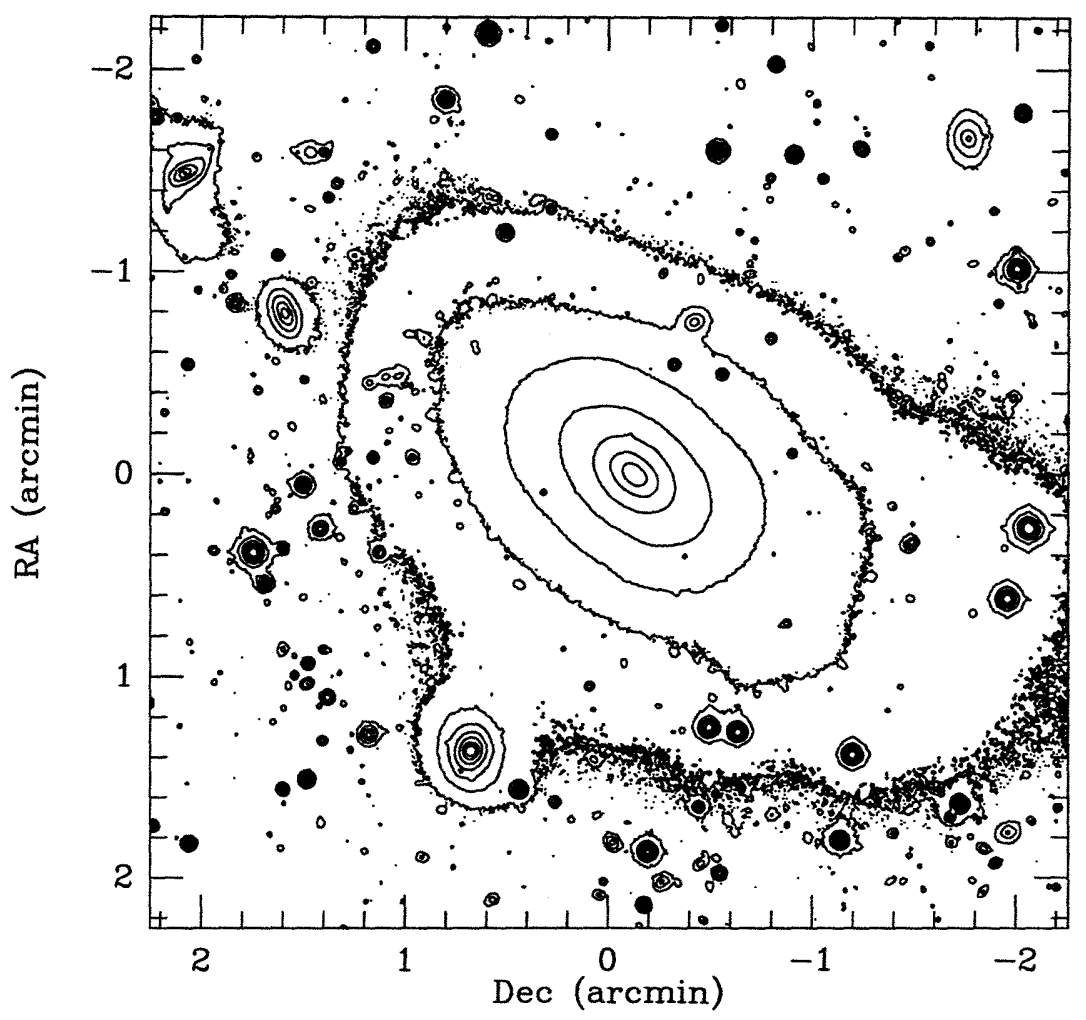

Figure 4-20: A2197-1.

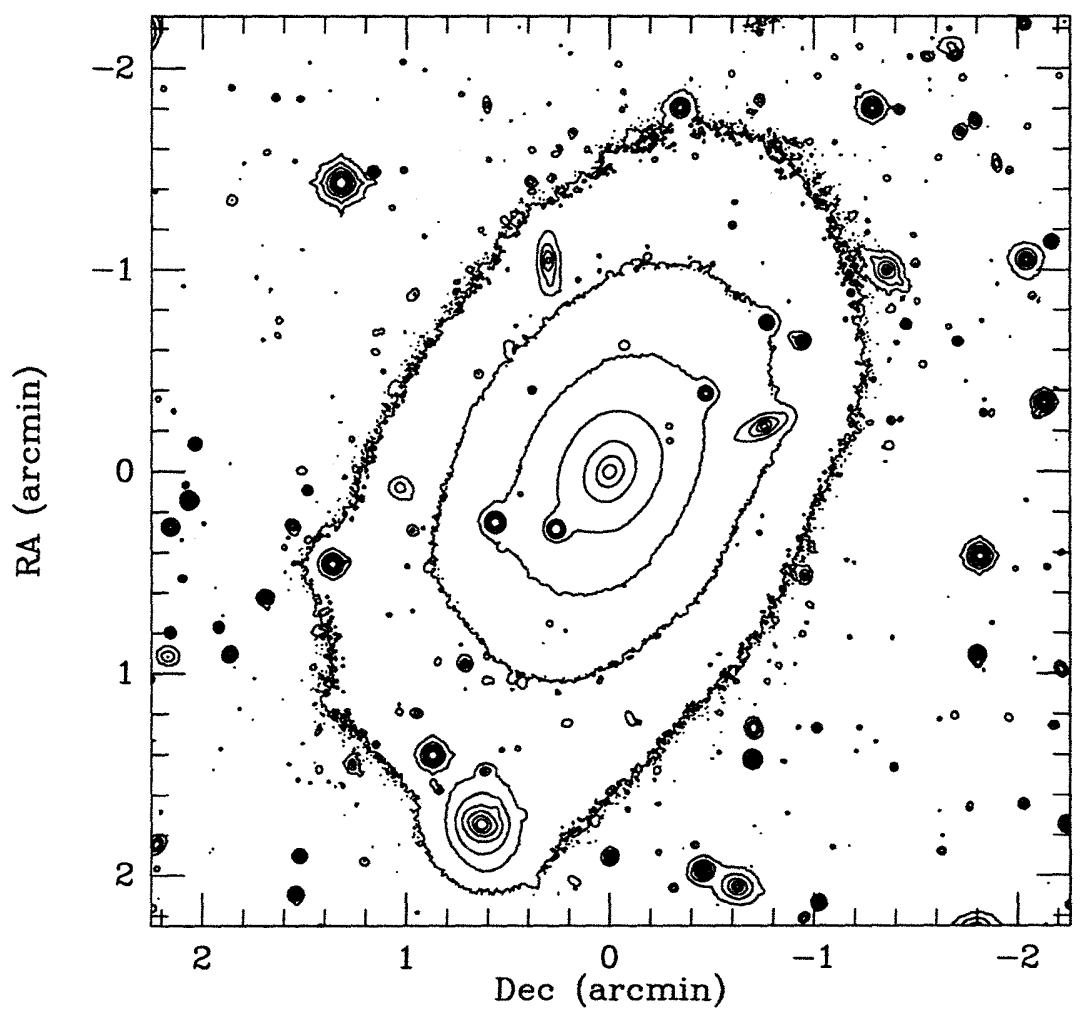

Figure 4-21: A2197-2. 


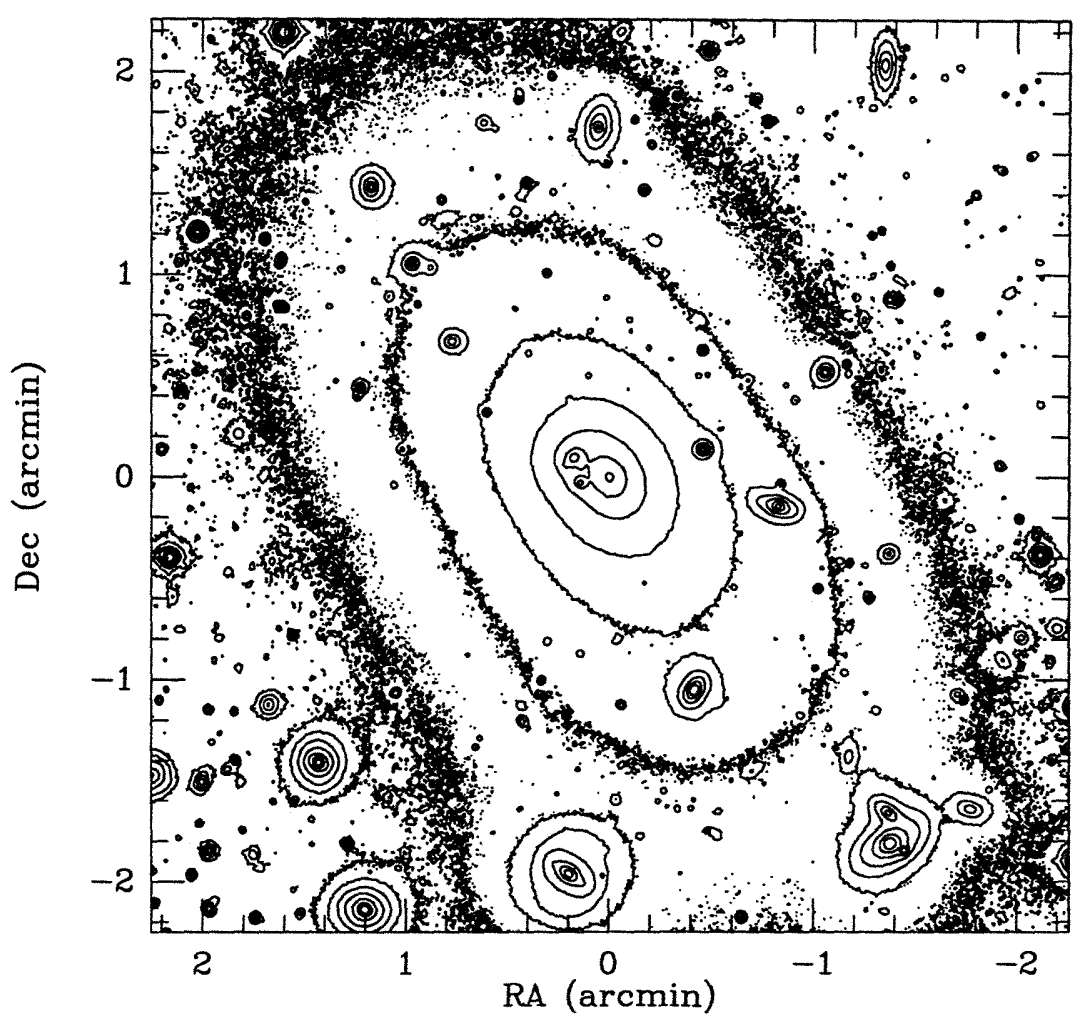

Figure 4-22: A2199-1.

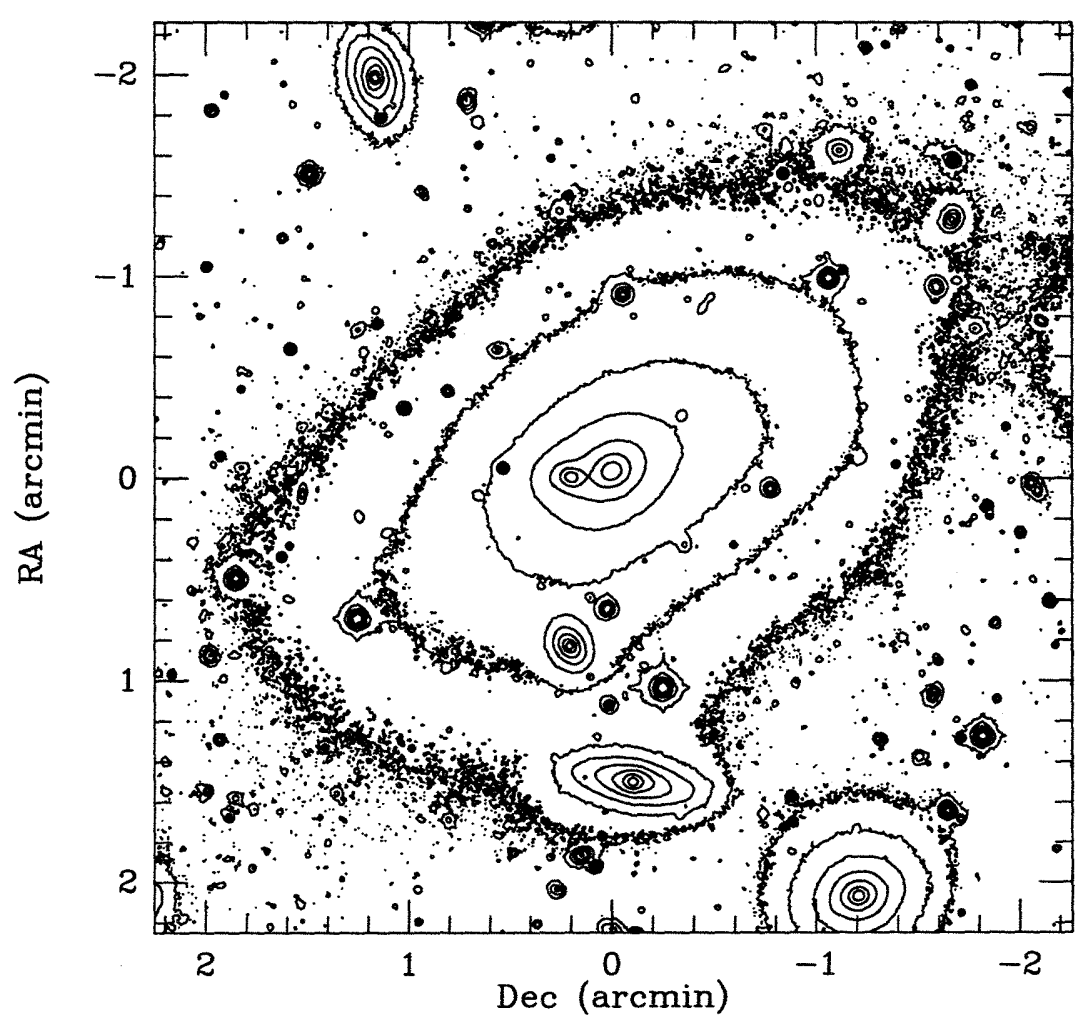

Figure 4-23: A2634-1. 


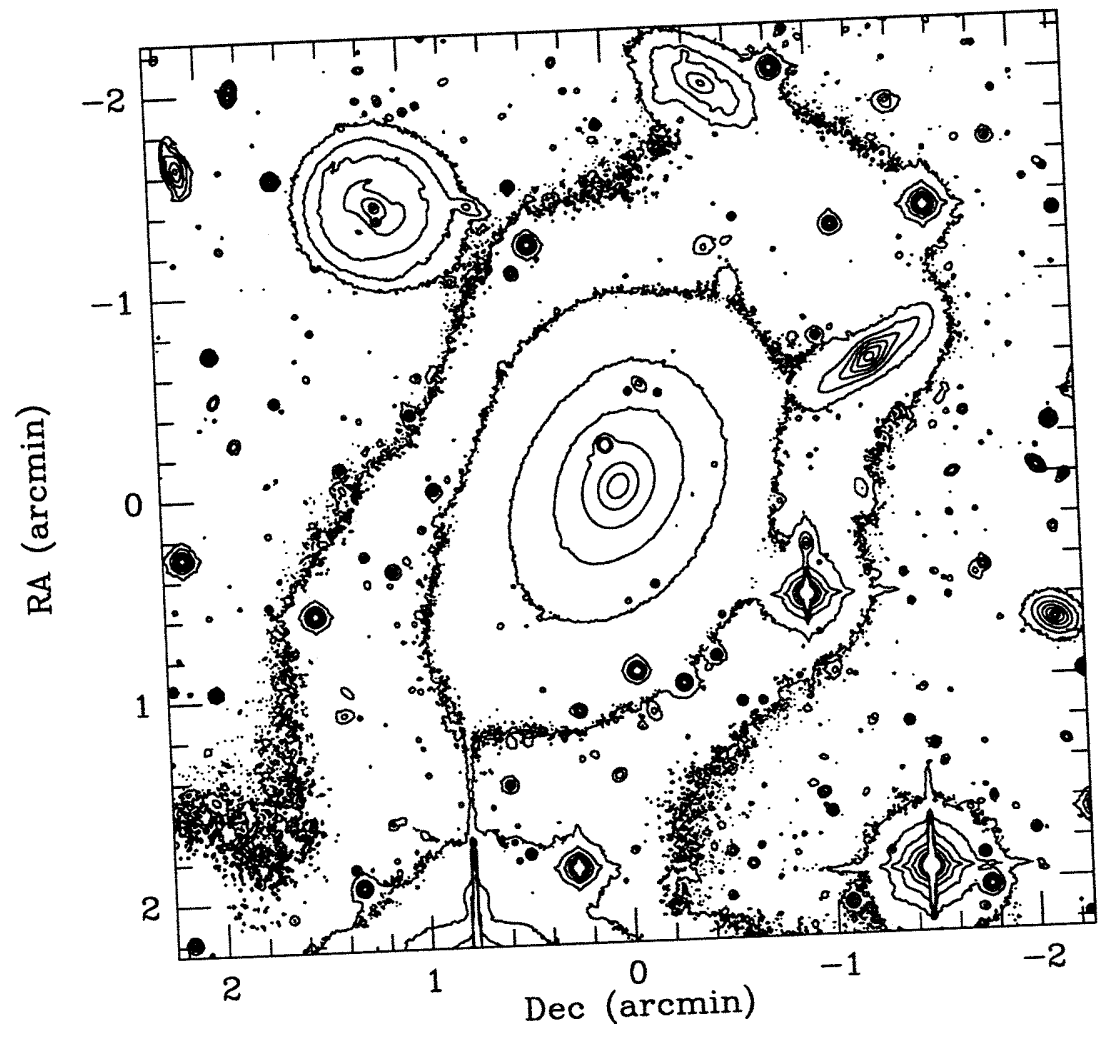

Figure 4-24: A2666-1. 


\subsubsection{Comments on Individual Galaxies}

Galaxies referred to below as "multiple nucleus BCGs" were in the Hoessel \& Schneider (1985) tabulation of 94 such galaxies. Velocities quoted for the secondary nuclei come from Tonry (1985), unless otherwise noted.

A262-1. NGC 708, the nearest galaxy in the sample, it has a central dust lane which noticeably distorts the innermost isophote in the contour plot. It has a very shallow falloff of surface brightness with metric radius, which among these galaxies is surpassed by only NGC 4874. It is not a $\mathrm{cD}$ galaxy, however, as it closely follows an $r^{1 / 4}$ law outside $\sim 0.5$. PL mistakenly call this galaxy NGC 705, which is actually the edge-on disk galaxy within its halo.

A347-1. NGC 910, also relatively nearby, is an undisturbed elliptical.

A397-1. The distorted isophotes probably indicate tidal interaction.

A539-1. A bright star to the west has masked out in the contour map.

A539-2. Although not chosen by either LP or Hoessel, Gunn, \& Thuan (1980) as the BCG, this galaxy is clearly nearer to the dynamical center of its cluster than is the official BCG.

A569-1. NGC 2329, though an excellent fit to an $r^{1 / 4}$ law, is remarkable for the fact that it shows significant major axis rotation (Fisher, Illingworth, \& Franx 1995).

A634-1. Low surface brightness shells, spray, and tidal distortions, primarily to the north and west, are present at the same distance as the companion to the southwest.

A779-1. NGC 2832, cD galaxy and a multiple nucleus BCG. As seen by Lauer (1988), there is a "dynamical friction wake" apparent when smooth models are subtracted, although the relative velocity of the secondary nucleus is $1692 \mathrm{~km} \mathrm{~s}^{-1}$. Further out, this galaxy shows little or no tidal disruption.

A999-1. No strong tidal signature of interaction with the companion to the southeast, even after smooth model subtraction. The companion galaxy itself is interacting strongly with its secondary nucleus $15^{\prime \prime}$ to the east of it.

A1016-1. The linear features in the contour plot are reflected light from a bright star. 
A1177-1. This galaxy has non-elliptical isophotes and shell-like features probably indicative of dynamical interaction; it bears a remarkable resemblance to A2634-1.

A1185-1. NGC 3550, this multiple nucleus BCG is a complex triple nucleus system in which the isophote centers first drift towards the southwest, then change sign and move significantly to the northeast. There are also strong tidal features including a dynamical wake (Lauer 1988). The nucleus to the northeast has a relative velocity of only $57 \mathrm{~km} \mathrm{~s}^{-1}$, while the one to the southwest has a relative velocity of $534 \mathrm{~km} \mathrm{~s}^{-1}$.

A1314-1. The galaxy is off center on the CCD chip to avoid the extremely bright star to its north. The feature $\gtrsim 1^{\prime}$ due west is a dusty, disturbed spiral.

A1367-1. NGC 3842, brightest galaxy in one of only 3 richness class 2 clusters in this sample. It does not appear to be interacting with any of the relatively bright nearby galaxies.

A1656-1. NGC 4889, multiple nucleus Coma BCG. The secondary nucleus disk galaxy has a relative velocity of $1178 \mathrm{~km} \mathrm{~s}^{-1}$ and does not appear to be interacting.

A1656-2. NGC 4874, the central cD galaxy in Coma, is popular among observers and neighboring galaxies alike.

A1656-3. NGC 4839, another Coma galaxy with a $\mathrm{cD}$ envelope, is unusual in that it is not centrally located in the cluster.

A2162-1. NGC 6086, a cD galaxy with some evidence of tidal interaction, including a shell-like feature about $2^{\prime}$ north. Unfortunately, this galaxy was observed at high $(\sim 1.5)$ airmass, and thus, its image has the worst seeing in the sample.

A2197-1. NGC 6173, an obviously disturbed galaxy, has inner parts which follow an $r^{1 / 4}$ law, apart from numerous shell which are visible when a smooth model is subtracted. Further out, as evident in the contour plot, there is an excess of light to the southeast and even some spiral structure (most apparent in the $23.5 \mathrm{mag} / \mathrm{arcsec}^{2}$ isophote). Perhaps a $\mathrm{cD}$ galaxy in the making?

A2197-2. NGC 6160, chosen as the BCG in this cluster by Hoessel, Gunn, \& Thuan (1980). Unlike NGC 6173, this galaxy is a nice elliptical without shells or obvious distortions. 
A2199-1. NGC 6166, the famous multiple nucleus cD galaxy, appears to be interacting with both the secondary nuclei to the northeast and southeast, as an apparent dynamical wake connects them. These nuclei have respective velocities of 1285 and $743 \mathrm{~km} \mathrm{~s}^{-1}$ relative to the primary. The primary has a significant amount of dust in the center $\sim 15^{\prime \prime}$, and when smooth models of the nuclei are subtracted, there is even a hint of a spiral residual pattern in these central regions.

A2634-1. NGC 7720, cD galaxy with tidal features, asymmetry, and nonconcentric isophotes. This galaxy was not in the Hoessel \& Schneider listing of multiple nucleus BCGs because it was not observed by them. It was studied by Lauer (1988) who concluded that the nuclei were interacting. However, these data show no strong tidal distortions at such small radii and subtraction of a smooth model leaves very little model residuals. Thus, the situation here is exactly the reverse of that in A779-1. The relative velocity of of the secondary nucleus is $997 \mathrm{~km} \mathrm{~s}^{-1}$ (Smith et al. 1985).

A2666-1. NGC 7768, another cD galaxy (but not studied by Schombert [1988]) with several close neighbors that appears to have outer isophote distortion, though some of the apparent distortion is due to the very bright star just off the image to the east. Like A569-1, this galaxy is unusual for a BCG in that it has significant rotation (Fisher et al. 1995). HST imaging shows that it also possesses a dusty nuclear disk (Grillmair et al. 1994).

\subsubsection{Galaxy Modeling and Subtraction}

Before proceeding to study the GC populations of these galaxies, it is necessary to model and subtract the galaxy light. This is done by masking out all the easily visible stars and smaller galaxies then modeling the light with a program called "elliprof" written by John Tonry for the SBF survey (Tonry et al. 1997). For many of these fields, several of the galaxies were modeled and subtracted using an iterative procedure as described in Chapter 2.

In some of the images, however, the multiple galaxies are comparable in size, close together, and show interaction. The best example of this is A539-2. For these galaxies, 


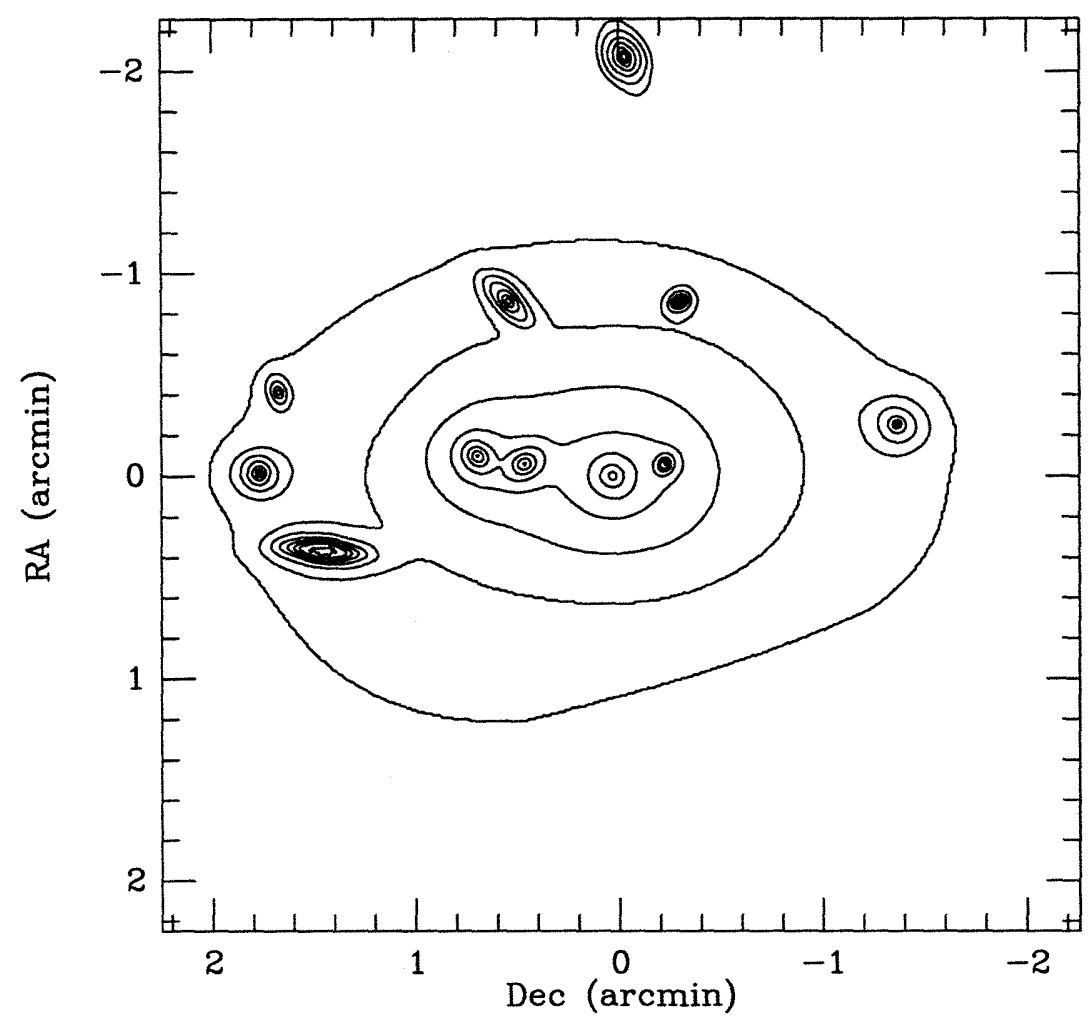

Figure 4-25: A539-2 Model.

elliprof could not be made to work on the first iteration; thus, I used the simultaneous nucleus fitting software known as "snuc" ( $s n \bar{u} k)$ which was also used by Lauer (1988) and LP. After simultaneously modeling and subtracting the primary galaxy and its secondary nuclei, I added the primary back in and could then successfully model it with elliprof. The same was done for the rest of the nuclei until all of them had been modeled with elliprof and subtracted. Often a second elliprof iteration was then required to minimize the model residuals.

The reason elliprof leaves smaller residuals than snuc is that the galaxies frequently show large departures from ellipticity, which elliprof is designed to handle via third, fourth, and sixth order Fourier terms, but snuc is not (there are only so many free parameters a program can have). Neither program fits disk galaxies or the centers of ellipticals well. Figures 4-25 and 4-26 show examples of some final galaxy models. The isophotes are drawn at the same scale and surface brightness levels as in the galaxy contour plots. 


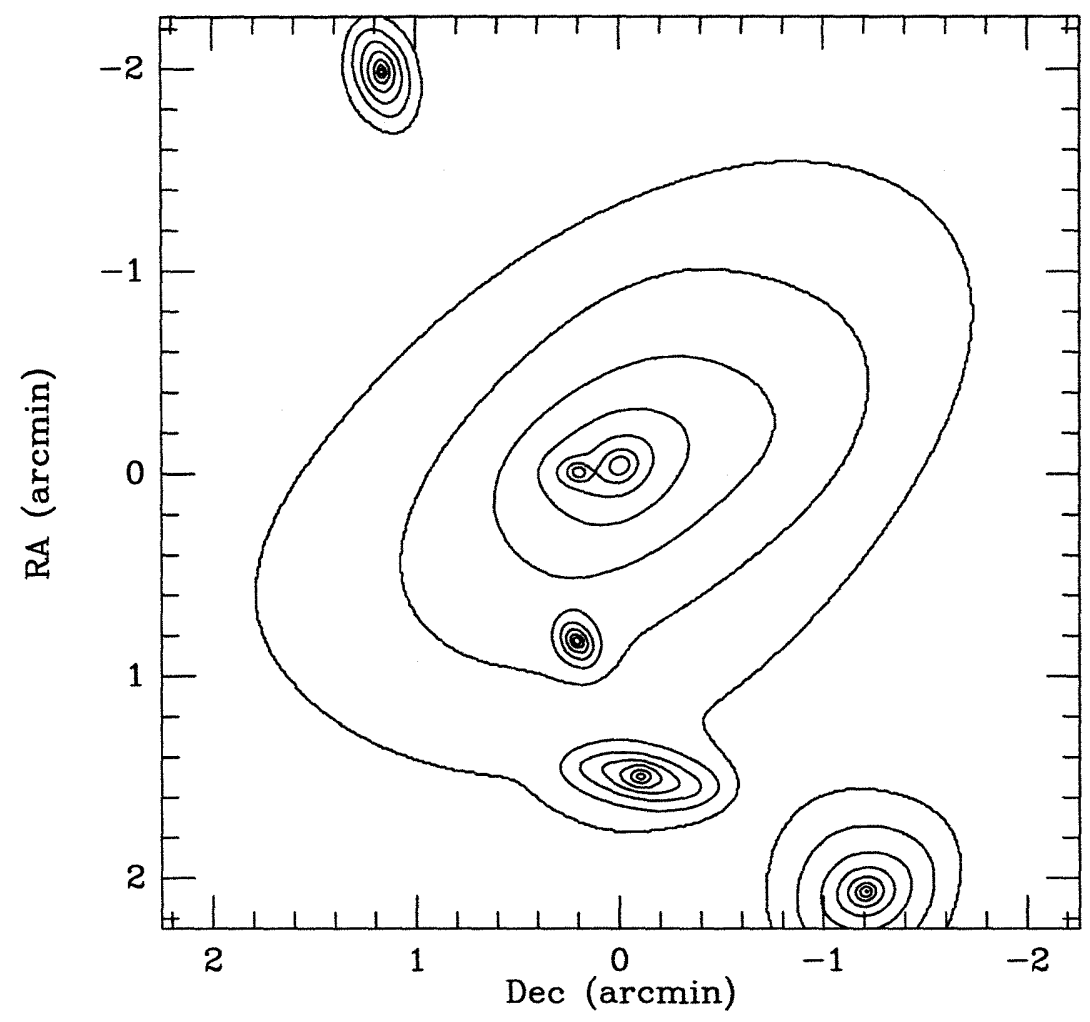

Figure 4-26: A2634-1 Model.

After subtracting the final galaxy model, all visible objects are masked in the image, and the large scale model residuals are fitted to a grid of spacing roughly 10 times the size of the PSF. A smooth model is then interpolated from this grid and subtracted from the image. The point source photometry and power spectrum measurements are carried out on this very flat "residual image." The rest of this chapter describes those reduction procedures.

\subsection{Point Source and Fluctuation Reductions}

\subsubsection{Finding Things with DoPhot}

As with the 0593 Coma galaxies, the automatic photometry program DoPhot (Schechter et al. 1993) was run on an integer version of each residual image in which saturated stars, "large" dwarf galaxies, and other bad features had been masked. Afterwards, I closely inspected DoPhot's model image (generated by taking the difference of the input and output images) to look for extended objects which had been fitted as tight 
clusters or strings of point sources. Objects larger than DoPhot's fit box cannot be fitted as single objects, but even some smaller ones refuse to conform to any reasonable model DoPhot might try. Thus, in all cases, it was necessary to mask out these problematic objects (which sometimes included residuals from the galaxy subtraction) and re-run DoPhot. Aside from these aberrant cases, DoPhot does a good job of distinguishing between point sources and extended objects, as tests performed by Ajhar, Blakeslee, \& Tonry (1994) showed. This proves to be a useful feature, as noted in Chapter 2.

DoPhot's fit magnitudes must then be calibrated onto an absolute scale. This is done by taking the median difference of the aperture and fit magnitudes among the $\lesssim 20$ brightest objects. Typical aperture corrections were $\sim 0.32 \mathrm{mag}$, with an uncertainty of about $0.02-0.03 \mathrm{mag}$, judging from the scatter in the brightest stars' aperture corrections. This is about twice as large as the photometric uncertainty from the standard star calibration. In one case (the A1314-1 field), the automatic procedure for selecting the aperture correction made a very bad choice, off by $0.08 \mathrm{mag}$, due the paucity of bright stars in the field. I discovered this discrepancy while doing the completeness tests described in the following section. Thereafter, I studiously checked all the aperture corrections that were used and found no other similar problems.

The total number of unique objects identified and fitted by DoPhot (i.e., types $1-4,7,9)$ in each field were listed in Table 4.5 . These numbers do not precisely reflect the relative densities of objects among the images because the final field size varies due to unequal telescope shifting, windowing, and masking (as well as different detectors in a few cases), but they convey a general impression of the number of objects present in each residual image.

\subsubsection{Completeness Tests}

In order to determine the completeness of DoPhot in finding point sources as a function of magnitude, a bright clean star was chosen from each field and cloned into a grid of stars with separation $\sim 10^{\prime \prime}$. The grid was then scaled 5-7 times in $0.5 \mathrm{mag}$ steps, with the noise in the grid increased appropriately for each scaling. The bright- 
est scaling was at $m=22.0-23.0$, depending on the depth of the image. Each scaled grid of stars was added to a separate copy of the real data, and DoPhot was run on the image; all parameters were set to the same values as in the original run. Then the results of each run were matched to the grid stars to determine the completeness and any possible magnitude bias. Table 4.6 displays the results of these $\sim 130$ DoPhot test runs.

For each galaxy image, the table lists all of the completeness tests that were performed, including: the intended, or fiducial, magnitude $m_{F}$ of the test; the median magnitude of the recovered stars $\langle m\rangle$; the difference, or bias, $\delta=\langle m\rangle-m_{F}$; the median uncertainty reported by DoPhot for the magnitudes of the recovered stars; the total number $\mathrm{N}_{\mathrm{add}}$ of grid stars added to the unmasked regions of the data image; the completeness fraction $f_{c}=\mathrm{N}_{\text {found }} / \mathrm{N}_{\mathrm{add}}$; and the statistical uncertainty in the completeness $\delta f_{c}=\left[f_{c}\left(1-f_{c}\right) / \mathrm{N}_{\mathrm{add}}\right]^{1 / 2}$ (Bolte 1989).

The table shows that $\delta$ becomes more negative as the test magnitudes increase. This is because the stars which land on negative noise fluctuations are more likely to be missed at fainter magnitudes, skewing the median, as noted by Schechter et al. (1993). For each set of tests, there may also be a systematic offset due to imperfect scaling of the star flux at the initial magnitude level. The clearest example of this can be seen in the tabulated results for A1367-1. Thus, the true bias at a given magnitude is better estimated by taking the difference of $\delta$ listed at that magnitude and $\delta$ listed for the brightest magnitude in the set. Calculated this way, it is always smaller than median uncertainty reported by DoPhot at the same magnitude.

The test results for each field were used in choosing the cutoff magnitude $m_{c}$ at which the point source completeness was $\sim 90 \%$ and the photometric error small. Actually, the completeness depends on radius, so typically two different cutoff magnitudes were used, with $m_{c}$ being 0.5 mag fainter outside the central $\sim 1^{\prime}$. For each separate region of the image, an appropriate uncertainty in the completeness was used, based on the number of added stars which landed in that region (and thus larger than the overall uncertainties listed in the table). 
Table 4.6: DoPhot Completeness Tests

\begin{tabular}{|c|c|c|c|c|c|c|c|}
\hline Galaxy & $m_{F}$ & $\langle m\rangle$ & $\delta$ & $\langle \pm\rangle$ & $\mathrm{N}_{\text {add }}$ & $f_{c}$ & \pm \\
\hline \multirow[t]{5}{*}{ A262-1 } & 22.5 & 22.49 & -0.01 & 0.03 & 490 & 0.986 & 0.005 \\
\hline & 23.0 & 22.99 & -0.01 & 0.05 & 490 & 0.973 & 0.007 \\
\hline & 23.5 & 23.48 & -0.02 & 0.07 & 490 & 0.963 & 0.008 \\
\hline & 24.0 & 23.97 & -0.03 & 0.11 & 490 & 0.931 & 0.011 \\
\hline & 24.5 & 24.43 & -0.07 & 0.17 & 490 & 0.806 & 0.018 \\
\hline \multirow[t]{5}{*}{ A347-1 } & 22.5 & 22.50 & 0.00 & 0.03 & 606 & 0.982 & 0.005 \\
\hline & 23.0 & 23.00 & 0.00 & 0.05 & 606 & 0.974 & 0.007 \\
\hline & 23.5 & 23.49 & -0.01 & 0.07 & 606 & 0.965 & 0.007 \\
\hline & 24.0 & 23.99 & -0.01 & 0.11 & 606 & 0.942 & 0.009 \\
\hline & 24.5 & 24.45 & -0.05 & 0.17 & 606 & 0.835 & 0.015 \\
\hline \multirow[t]{6}{*}{ A347-1 } & 22.5 & 22.48 & -0.02 & 0.02 & 694 & 0.996 & 0.002 \\
\hline & 23.0 & 22.97 & -0.03 & 0.03 & 694 & 0.990 & 0.004 \\
\hline & 23.5 & 23.47 & -0.03 & 0.05 & 694 & 0.986 & 0.005 \\
\hline & 24.0 & 23.96 & -0.04 & 0.08 & 694 & 0.965 & 0.007 \\
\hline & 24.5 & 24.44 & -0.06 & 0.12 & 694 & 0.947 & 0.009 \\
\hline & 25.0 & 24.90 & -0.10 & 0.18 & 694 & 0.869 & 0.013 \\
\hline \multirow[t]{6}{*}{ A $397-1$} & 23.0 & 22.98 & -0.02 & 0.02 & 652 & 0.995 & 0.003 \\
\hline & 23.5 & 23.47 & -0.03 & 0.03 & 652 & 0.995 & 0.003 \\
\hline & 24.0 & 23.97 & -0.03 & 0.05 & 652 & 0.989 & 0.004 \\
\hline & 24.5 & 24.46 & -0.04 & 0.08 & 652 & 0.977 & 0.006 \\
\hline & 25.0 & 24.95 & -0.05 & 0.13 & 652 & 0.968 & 0.007 \\
\hline & 25.5 & 25.40 & -0.10 & 0.19 & 652 & 0.837 & 0.014 \\
\hline \multirow[t]{5}{*}{ A539-1 } & 23.0 & 23.00 & 0.00 & 0.03 & 541 & 0.976 & 0.007 \\
\hline & 23.5 & 23.49 & -0.01 & 0.04 & 541 & 0.967 & 0.008 \\
\hline & 24.0 & 23.99 & -0.01 & 0.06 & 541 & 0.941 & 0.010 \\
\hline & 24.5 & 24.48 & -0.02 & 0.10 & 541 & 0.924 & 0.011 \\
\hline & 25.0 & 24.95 & -0.05 & 0.15 & 541 & 0.845 & 0.016 \\
\hline \multirow[t]{5}{*}{ A539-2 } & 23.0 & 22.98 & -0.02 & 0.03 & 432 & 0.981 & 0.006 \\
\hline & 23.5 & 23.48 & -0.02 & 0.04 & 432 & 0.972 & 0.008 \\
\hline & 24.0 & 23.97 & -0.03 & 0.07 & 432 & 0.951 & 0.010 \\
\hline & 24.5 & 24.45 & -0.05 & 0.10 & 432 & 0.917 & 0.013 \\
\hline & 25.0 & 24.92 & -0.08 & 0.15 & 432 & 0.840 & 0.018 \\
\hline \multirow[t]{5}{*}{ A569-1 } & 22.5 & 22.48 & -0.02 & 0.02 & 561 & 0.986 & 0.005 \\
\hline & 23.0 & 22.98 & -0.02 & 0.03 & 561 & 0.971 & 0.007 \\
\hline & 23.5 & 23.47 & -0.03 & 0.05 & 561 & 0.950 & 0.009 \\
\hline & 24.0 & 23.97 & -0.03 & 0.08 & 561 & 0.936 & 0.010 \\
\hline & 24.5 & 24.45 & -0.05 & 0.12 & 561 & 0.913 & 0.012 \\
\hline \multirow{6}{*}{ A569-1 } & 22.0 & 21.99 & -0.01 & 0.02 & 658 & 0.982 & 0.005 \\
\hline & 22.5 & 22.48 & -0.02 & 0.03 & 658 & 0.979 & 0.006 \\
\hline & 23.0 & 22.97 & -0.03 & 0.05 & 658 & 0.968 & 0.007 \\
\hline & 23.5 & 23.47 & -0.03 & 0.07 & 658 & 0.948 & 0.009 \\
\hline & 24.0 & 23.95 & -0.05 & 0.11 & 658 & 0.932 & 0.010 \\
\hline & 24.5 & 24.39 & -0.11 & 0.17 & 658 & 0.783 & 0.016 \\
\hline
\end{tabular}


Table 4.6-Continued.

\begin{tabular}{|c|c|c|c|c|c|c|c|}
\hline Galaxy & $m_{F}$ & $\langle m\rangle$ & $\delta$ & $\langle \pm\rangle$ & $\mathrm{N}_{\text {add }}$ & $f_{c}$ & \pm \\
\hline \multirow[t]{6}{*}{ A634-1 } & 23.0 & 22.99 & -0.01 & 0.02 & 578 & 0.991 & 0.004 \\
\hline & 23.5 & 23.48 & -0.02 & 0.03 & 578 & 0.984 & 0.005 \\
\hline & 24.0 & 23.98 & -0.02 & 0.05 & 578 & 0.960 & 0.008 \\
\hline & 24.5 & 24.47 & -0.03 & 0.08 & 578 & 0.952 & 0.009 \\
\hline & 25.0 & 24.95 & -0.05 & 0.12 & 578 & 0.924 & 0.011 \\
\hline & 25.5 & 25.41 & -0.09 & 0.18 & 578 & 0.808 & 0.016 \\
\hline \multirow[t]{5}{*}{ A779-1 } & 23.0 & 22.99 & -0.01 & 0.02 & 655 & 0.991 & 0.004 \\
\hline & 23.5 & 23.48 & -0.02 & 0.04 & 655 & 0.989 & 0.004 \\
\hline & 24.0 & 23.97 & -0.03 & 0.06 & 655 & 0.977 & 0.006 \\
\hline & 24.5 & 24.47 & -0.03 & 0.09 & 655 & 0.969 & 0.007 \\
\hline & 25.0 & 24.95 & -0.05 & 0.14 & 655 & 0.936 & 0.010 \\
\hline \multirow[t]{5}{*}{ A999-1 } & 23.5 & 23.48 & -0.02 & 0.03 & 593 & 0.978 & 0.006 \\
\hline & 24.0 & 23.98 & -0.02 & 0.04 & 593 & 0.971 & 0.007 \\
\hline & 24.5 & 24.48 & -0.02 & 0.06 & 593 & 0.960 & 0.008 \\
\hline & 25.0 & 24.97 & -0.03 & 0.10 & 593 & 0.929 & 0.011 \\
\hline & 25.5 & 25.44 & -0.06 & 0.15 & 593 & 0.895 & 0.013 \\
\hline \multirow[t]{5}{*}{ A1016-1 } & 23.5 & 23.47 & -0.03 & 0.03 & 540 & 0.983 & 0.006 \\
\hline & 24.0 & 23.98 & -0.02 & 0.04 & 540 & 0.978 & 0.006 \\
\hline & 24.5 & 24.48 & -0.02 & 0.06 & 540 & 0.969 & 0.008 \\
\hline & 25.0 & 24.98 & -0.02 & 0.10 & 540 & 0.952 & 0.009 \\
\hline & 25.5 & 25.47 & -0.03 & 0.16 & 540 & 0.911 & 0.012 \\
\hline \multirow[t]{6}{*}{ A1177-1 } & 23.0 & 22.97 & -0.03 & 0.02 & 653 & 0.989 & 0.004 \\
\hline & 23.5 & 23.47 & -0.03 & 0.03 & 653 & 0.985 & 0.005 \\
\hline & 24.0 & 23.97 & -0.03 & 0.04 & 653 & 0.974 & 0.006 \\
\hline & 24.5 & 24.46 & -0.04 & 0.07 & 653 & 0.972 & 0.006 \\
\hline & 25.0 & 24.94 & -0.06 & 0.10 & 653 & 0.946 & 0.009 \\
\hline & 25.5 & 25.42 & -0.08 & 0.16 & 653 & 0.896 & 0.012 \\
\hline \multirow[t]{7}{*}{ A1185-1 } & 22.5 & 22.51 & +0.01 & 0.02 & 591 & 0.997 & 0.002 \\
\hline & 23.0 & 22.97 & -0.03 & 0.02 & 591 & 0.997 & 0.002 \\
\hline & 23.5 & 23.47 & -0.03 & 0.04 & 591 & 0.993 & 0.003 \\
\hline & 24.0 & 23.96 & -0.04 & 0.06 & 591 & 0.990 & 0.004 \\
\hline & 24.5 & 24.45 & -0.05 & 0.09 & 591 & 0.975 & 0.006 \\
\hline & 25.0 & 24.95 & -0.05 & 0.14 & 591 & 0.949 & 0.009 \\
\hline & 25.5 & 25.34 & -0.16 & 0.20 & 591 & 0.711 & 0.019 \\
\hline \multirow[t]{6}{*}{ A1314-1 } & 23.0 & 22.97 & -0.03 & 0.02 & 742 & 0.987 & 0.004 \\
\hline & 23.5 & 23.46 & -0.04 & 0.03 & 742 & 0.987 & 0.004 \\
\hline & 24.0 & 23.94 & -0.06 & 0.04 & 742 & 0.978 & 0.005 \\
\hline & 24.5 & 24.44 & -0.06 & 0.06 & 742 & 0.961 & 0.007 \\
\hline & 25.0 & 24.92 & -0.08 & 0.10 & 742 & 0.951 & 0.008 \\
\hline & 25.5 & 25.39 & -0.11 & 0.15 & 742 & 0.910 & 0.011 \\
\hline
\end{tabular}


Table 4.6-Continued.

\begin{tabular}{|c|c|c|c|c|c|c|c|}
\hline Galaxy & $m_{F}$ & $\langle m\rangle$ & $\delta$ & $\langle \pm\rangle$ & $\mathrm{N}_{\text {add }}$ & $f_{c}$ & \pm \\
\hline \multirow[t]{5}{*}{$\mathrm{A} 1367-1$} & 23.0 & 22.96 & -0.04 & 0.03 & 712 & 0.990 & 0.004 \\
\hline & 23.5 & 23.46 & -0.04 & 0.04 & 712 & 0.987 & 0.004 \\
\hline & 24.0 & 23.95 & -0.05 & 0.06 & 712 & 0.980 & 0.005 \\
\hline & 24.5 & 24.44 & -0.06 & 0.10 & 712 & 0.968 & 0.007 \\
\hline & 25.0 & 24.91 & -0.09 & 0.15 & 712 & 0.945 & 0.009 \\
\hline \multirow[t]{6}{*}{ A1656-3 } & 22.5 & 22.48 & -0.02 & 0.02 & 672 & 0.997 & 0.002 \\
\hline & 23.0 & 22.96 & -0.04 & 0.03 & 672 & 0.994 & 0.003 \\
\hline & 23.5 & 23.45 & -0.05 & 0.05 & 672 & 0.987 & 0.004 \\
\hline & 24.0 & 23.92 & -0.08 & 0.08 & 672 & 0.973 & 0.006 \\
\hline & 24.5 & 24.42 & -0.08 & 0.13 & 672 & 0.966 & 0.007 \\
\hline & 25.0 & 24.87 & -0.13 & 0.19 & 672 & 0.836 & 0.014 \\
\hline \multirow[t]{6}{*}{ A2162-1 } & 22.5 & 22.48 & -0.02 & 0.02 & 664 & 0.988 & 0.004 \\
\hline & 23.0 & 22.97 & -0.03 & 0.03 & 664 & 0.976 & 0.006 \\
\hline & 23.5 & 23.47 & -0.03 & 0.05 & 664 & 0.965 & 0.007 \\
\hline & 24.0 & 23.95 & -0.05 & 0.08 & 664 & 0.941 & 0.009 \\
\hline & 24.5 & 24.43 & -0.07 & 0.12 & 664 & 0.916 & 0.011 \\
\hline & 25.0 & 24.86 & -0.14 & 0.17 & 664 & 0.729 & 0.017 \\
\hline \multirow[t]{6}{*}{ A2197-1 } & 22.5 & 22.49 & -0.01 & 0.02 & 677 & 0.996 & 0.003 \\
\hline & 23.0 & 22.99 & -0.01 & 0.03 & 677 & 0.990 & 0.004 \\
\hline & 23.5 & 23.48 & -0.02 & 0.04 & 677 & 0.981 & 0.005 \\
\hline & 24.0 & 23.97 & -0.03 & 0.07 & 677 & 0.963 & 0.007 \\
\hline & 24.5 & 24.46 & -0.04 & 0.10 & 677 & 0.944 & 0.009 \\
\hline & 25.0 & 24.93 & -0.07 & 0.16 & 677 & 0.855 & 0.014 \\
\hline \multirow[t]{6}{*}{ A2197-2 } & 23.0 & 22.99 & -0.01 & 0.02 & 681 & 0.990 & 0.004 \\
\hline & 23.5 & 23.48 & -0.02 & 0.03 & 681 & 0.985 & 0.005 \\
\hline & 24.0 & 23.98 & -0.02 & 0.05 & 681 & 0.981 & 0.005 \\
\hline & 24.5 & 24.47 & -0.03 & 0.08 & 681 & 0.968 & 0.007 \\
\hline & 25.0 & 24.95 & -0.05 & 0.12 & 681 & 0.941 & 0.009 \\
\hline & 25.5 & 25.40 & -0.10 & 0.17 & 681 & 0.840 & 0.014 \\
\hline \multirow[t]{6}{*}{ A2199-1 } & 21.5 & 21.51 & +0.01 & 0.02 & 1603 & 0.993 & 0.002 \\
\hline & 22.0 & 22.01 & +0.01 & 0.03 & 1603 & 0.990 & 0.002 \\
\hline & 22.5 & 22.50 & 0.00 & 0.04 & 1603 & 0.981 & 0.003 \\
\hline & 23.0 & 23.00 & 0.00 & 0.06 & 1603 & 0.968 & 0.004 \\
\hline & 23.5 & 23.49 & -0.01 & 0.10 & 1603 & 0.958 & 0.005 \\
\hline & 24.0 & 23.97 & -0.03 & 0.15 & 1603 & 0.918 & 0.007 \\
\hline \multirow[t]{5}{*}{ A2634-1 } & 22.5 & 22.49 & -0.01 & 0.02 & 701 & 0.989 & 0.004 \\
\hline & 23.0 & 22.99 & -0.01 & 0.04 & 701 & 0.987 & 0.004 \\
\hline & 23.5 & 23.49 & -0.01 & 0.06 & 701 & 0.981 & 0.005 \\
\hline & 24.0 & 23.98 & -0.02 & 0.09 & 701 & 0.971 & 0.006 \\
\hline & 24.5 & 24.47 & -0.03 & 0.14 & 701 & 0.956 & 0.008 \\
\hline
\end{tabular}


Table 4.6-Continued.

\begin{tabular}{cccccccc}
\hline \hline Galaxy & $m_{F}$ & $\langle m\rangle$ & $\delta$ & $\langle \pm\rangle$ & $\mathrm{N}_{\text {add }}$ & $f_{c}$ & \pm \\
\hline A2666-1 & 22.5 & 22.50 & 0.00 & 0.02 & 457 & 0.998 & 0.002 \\
& 23.0 & 23.00 & 0.00 & 0.03 & 457 & 0.991 & 0.004 \\
& 23.5 & 23.50 & 0.00 & 0.05 & 457 & 0.987 & 0.005 \\
& 24.0 & 23.99 & -0.01 & 0.07 & 457 & 0.965 & 0.009 \\
& 24.5 & 24.47 & -0.03 & 0.11 & 457 & 0.958 & 0.009 \\
& 25.0 & 24.94 & -0.06 & 0.17 & 457 & 0.899 & 0.014 \\
\hline \hline
\end{tabular}

\subsubsection{Measuring the Power Spectra}

The power spectrum analysis used here is the same as in Chapter 2 and is described in detail by Tonry et al. 1990 and Jacoby et al. (1992). This section provides a summary and shows some of the power spectra from this data set. The next chapter discusses how these measurements are used in conjunction with the point source counts to derive information about the globular clusters.

After all objects brighter than the cutoff magnitude $m_{c}$ are masked out, the power spectrum of the masked data $P(k)$ is modeled as a linear function of the "expectation power spectrum" $E(k)$ :

$$
P(k)=P_{0} \times E(k)+P_{1} .
$$

$E(k)$ is computed as the convolution of the power spectra of the PSF and the window function of the mask. (The mask gets multiplied in image space, convolved in Fourier space.) $P_{0}$ is called the fluctuation power, the spatial variance in intensity which has been convolved with the PSF and therefore must have originated above the atmosphere. $P_{1}$ is the white noise component, which must be overcome through a propitious combination of exposure time, seeing, and a dark sky background. The star used for modeling the power spectrum of the PSF in each image was the same one used for doing the completeness tests.

The upper panels of Figures 4-27 through 4-31 show several examples of power spectra from the $\mathrm{c} 2$ and $\mathrm{c} 3$ regions (defined in Chapter 2) of the galaxy images. They range in quality from good (Figure 4-27) to poor (Figure 4-28), with a few in between. 
Over 160 power spectrum fits were performed on these data, so the ones shown here represent only $\sim 3 \%$ of the total. In the figures, the power has been divided by the mean galaxy intensity in order to make the numbers more wieldy. There is often excess power at low wavenumbers due to shells, tidal distortions, etc. which were not removed by the modeling described in $\S$ 4.3.5.

To deal with this problem, the low wavenumbers are not used in the power spectrum fits which determine $P_{0}$. The lower panels of the figures show the fitted $P_{0}$ as a function of the starting wavenumber of the fit (the fits are done all the way out, with the high wavenumbers providing a good handle on $P_{1}$ ). The point at which the fitted $P_{0}$ vs. wavenumber relation flattens out is taken as the initial starting point for fitting $P_{0}$. The power spectrum is then fitted with different starting wavenumbers ranging out to about twice this initial starting point. For instance, in the $256^{2}$ c2 regions, the range of starting wavenumbers for the fits might be $16-32$, and for the $512^{2}$ c3 regions, it might be 32-64. The weighted average of the fitted $P_{0}$ values for this range in the starting wavenumber is then taken, with the rms variance in $P_{0}$ over all the fits is included in the final uncertainty. The dashed lines in the bottom figure panels indicate the final values of $P_{0}$ derived in this way.

The measurements of the fluctuation powers $P_{0}$ are tabulated along with the measured point source number densities in the following chapter when we discuss background corrections. We then describe how the corrected measurements are used to derive information on the properties of the GCSs in these galaxies. New correlations of GCS properties with galaxy and cluster properties are then presented. The chapter concludes with a detailed discussion of the implications of our results. 


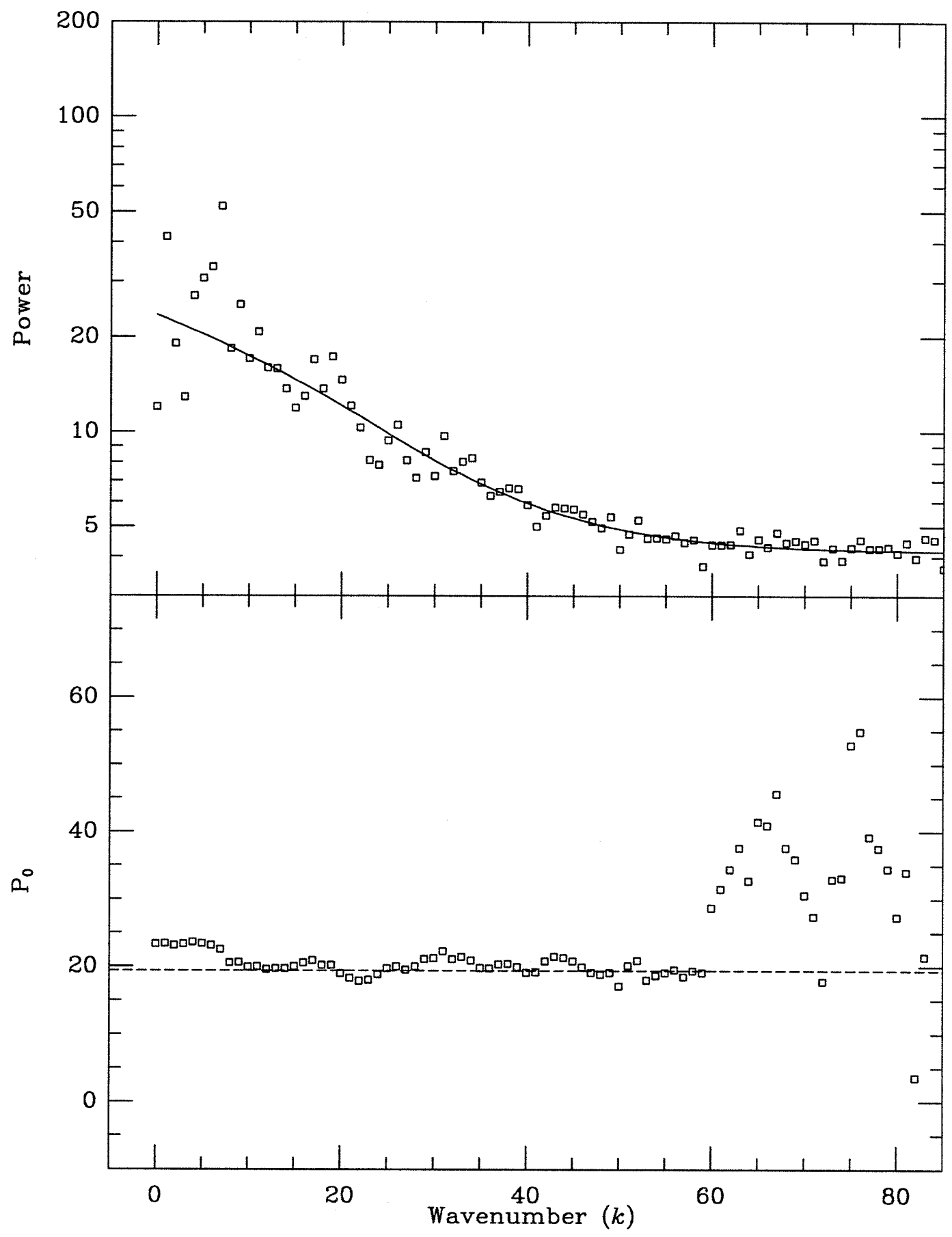

Figure 4-27: A347-1 Region 2 Power Spectrum 


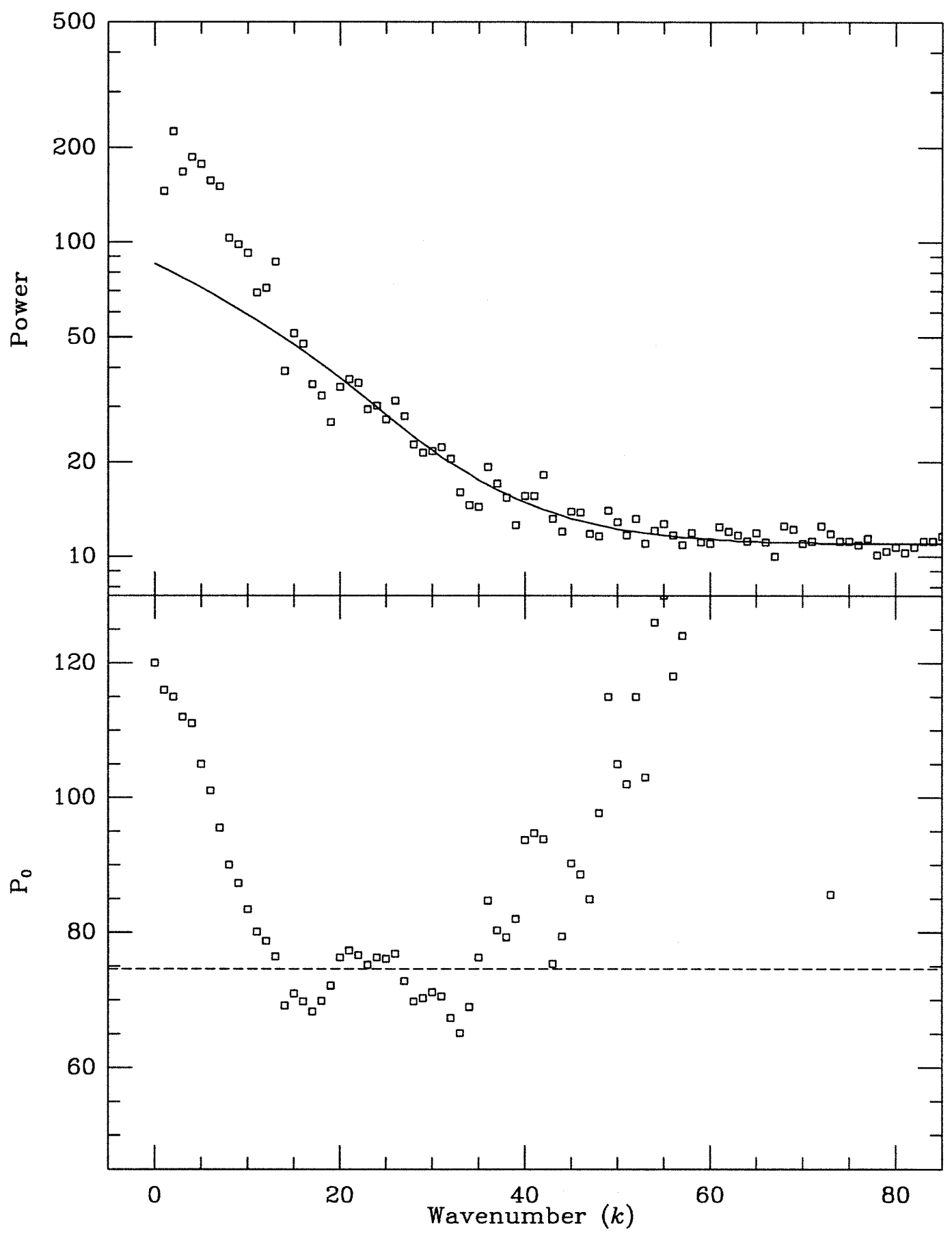

Figure 4-28: A2634-1 Region 2 Power Spectrum 


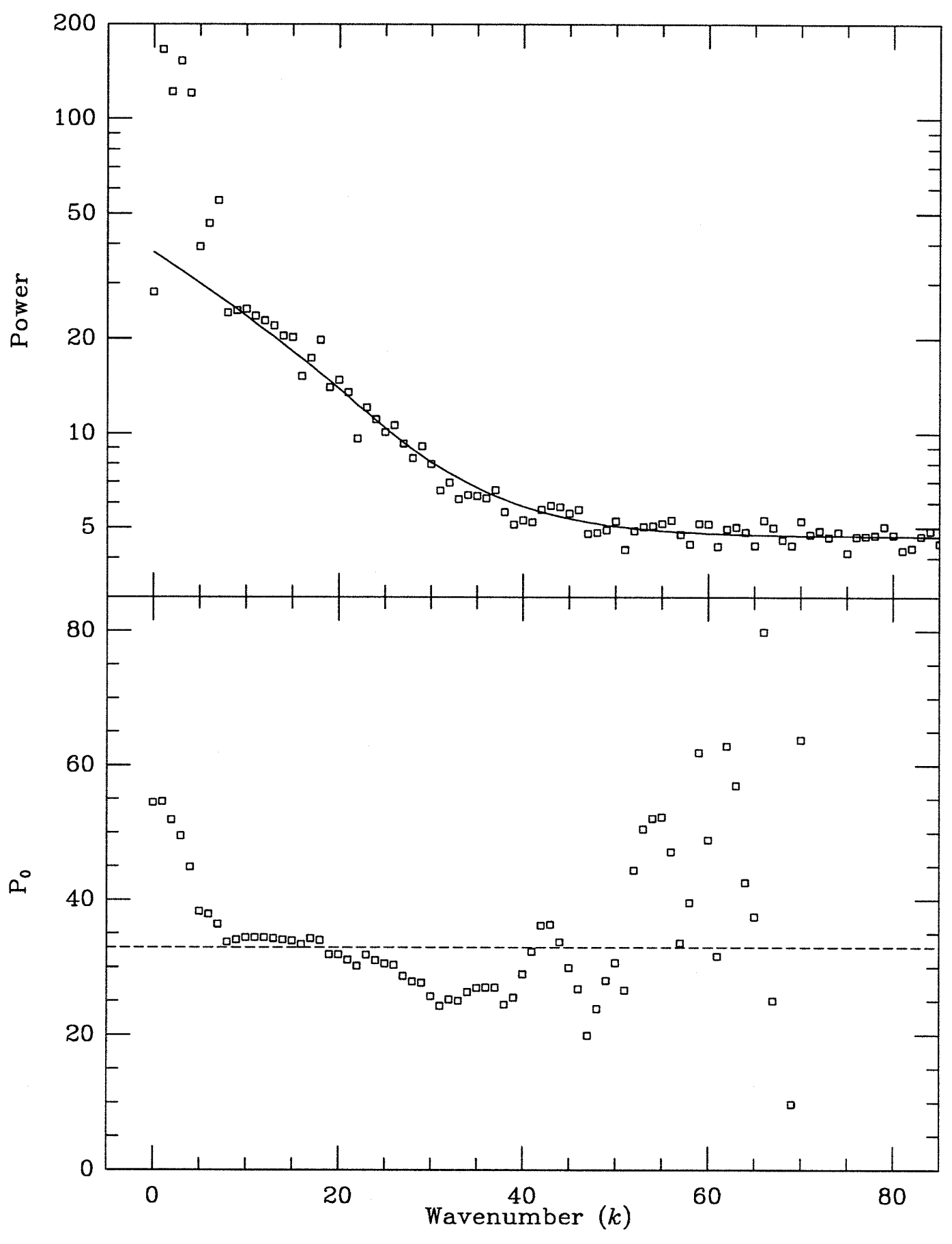

Figure 4-29: A2199-1 Region 2 Power Spectrum 


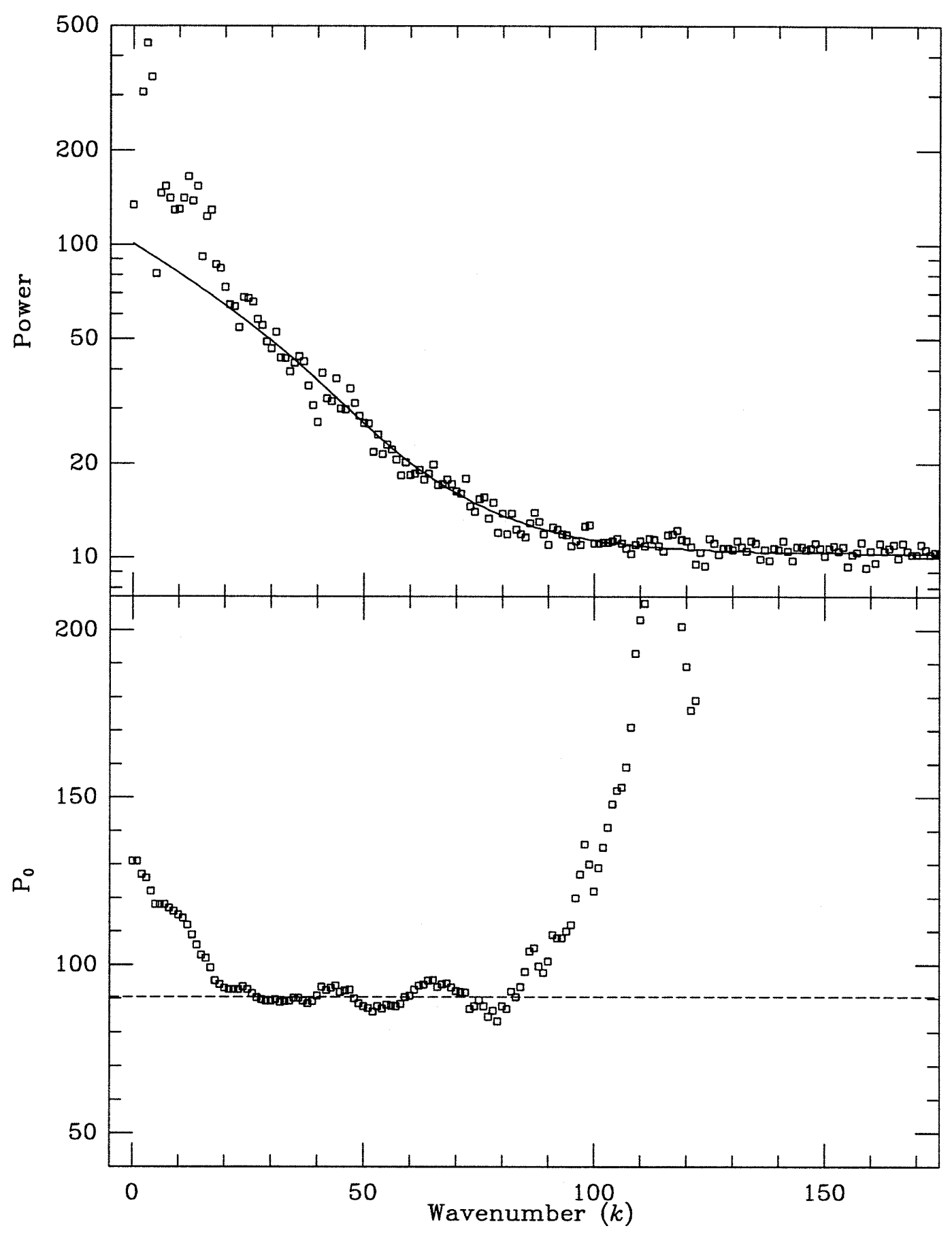

Figure 4-30: A2197-2 Region 3 Power Spectrum 


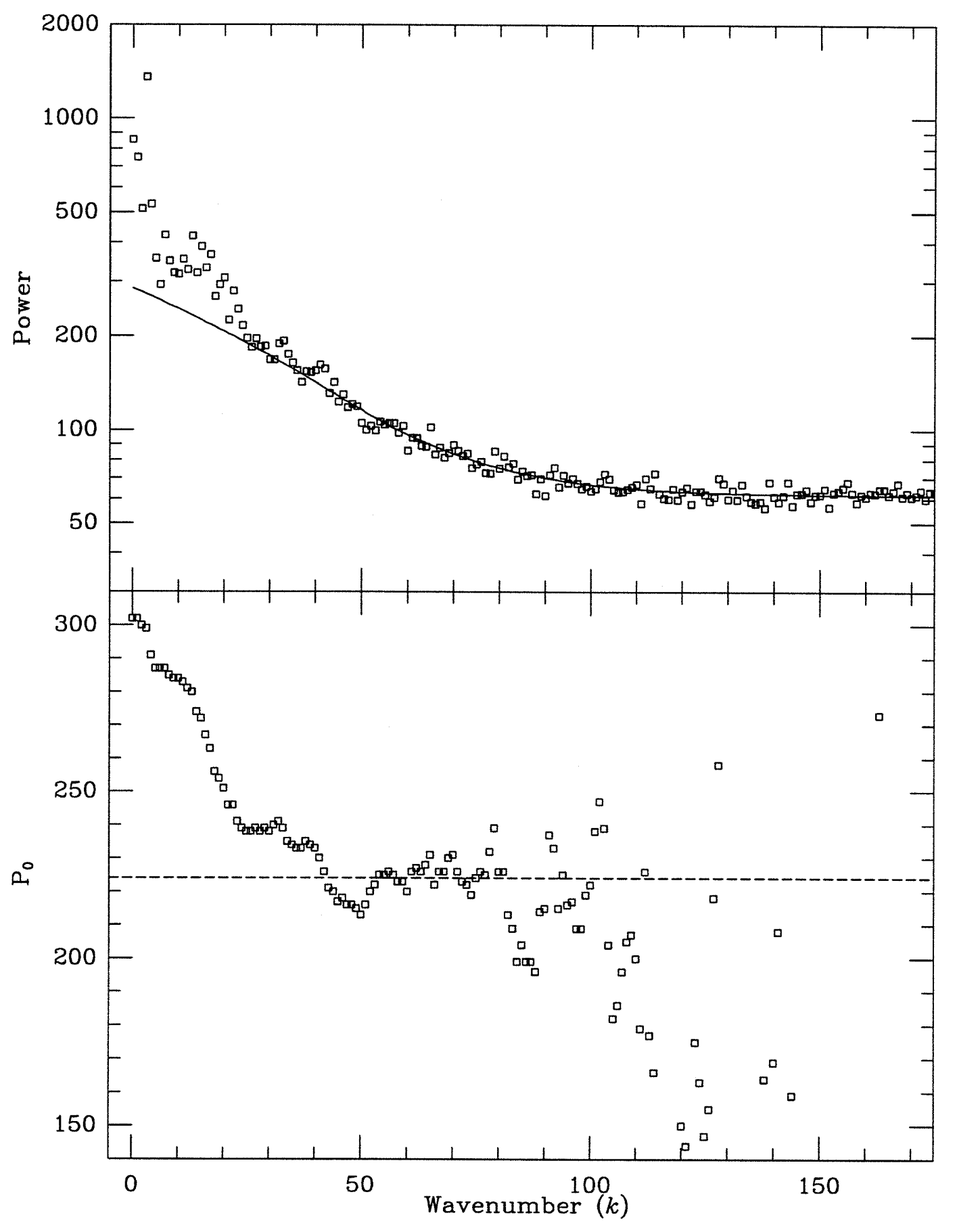

Figure 4-31: A1185-1 Region 3 Power Spectrum 


\subsection{References}

Abell, G. O. 1958, ApJS, 3, 211

Abell, G. O., Corwin H. G. Jr. \& Olowin, R. P. 1989, ApJS, 70, 1

Ajhar, E. A., Blakeslee, J. P., \& Tonry, J. L. 1994, AJ, 108, 2087

Blakeslee, J. P. \& Tonry, J. L. 1995, ApJ, 442, 579

Bolte, M. 1989, ApJ, 341, 168

Burstein, D. \& Heiles, C. 1984, ApJS, 54, 33

Dreyer, J. L. E. 1888, MmRAS, 49, 1

Dreyer, J. L. E. 1908, MmRAS, 59, 105

Fisher, D., Illingworth, G., \& Franx, M. 1995, ApJ, 438, 539

Grillmair, C. J., Faber, S. M., Lauer, T. R., Baum, W. A., Lynds, R. C., O'neil, E. J. Jr., \& Shaya, E. J. 1994, ApJ, 422, 9

Jacoby, G. H., Branch, D., Ciardullo, R., Davies, R. L., Harris, W. E., Pierce, M. J., Pritchet, C. J., Tonry, J. L., \& Welch, D. L. 1992, PASP, 104, 599

Jones, C. \& Forman, W. 1996, in preparation, almost ready for ApJ submission

Hoessel, J. G. 1980, ApJ, 241, 493

Hoessel, J. G., Gunn, J. E., \& Thuan T. X. 1980, ApJ, 241, 486

Hoessel, J. G. \& Schneider, D. P. 1985, AJ, 90, 1648 (HS)

Landolt, A. U. 1992, AJ, 104, 340

Lauer, T. R. 1988, ApJ, 325,49

Lauer, T. R. \& Postman, M. 1994, ApJ, 425, 418 (LP)

Nilson, P. 1973, Uppsala General Catalogue of Galaxies (Uppsala: Reg. Soc. Scient. Ups.)

Postman, M. 1996, private communication

Postman, M. \& Lauer, T. R. 1995, ApJ, 440, 28 (PL)

Schechter, P. L., Mateo, M., \& Saha A. 1993, PASP, 105, 1342

Schombert, J. M. 1988, ApJ, 328, 475

Struble, M. F. \& Rood, H. J. 1987 ApJS, 63, 555

Smith, R. M., Efstathiou, G., Ellis, R. S., Frenk, C. S., \& Valentijn, E. A. 1985, 
MNRAS, 216, 71P

Tonry, J. L. 1985, AJ, 90, 2431

Tonry, J. L. 1987, in Structure and Dynamics of Elliptical Galaxies, ed. T. de Zeeuw (Dordrecht: D. Reidel)

Tonry, J. L., Ajhar, E. A., \& Luppino, G. A. 1990, AJ, 100, 1416

Tonry, J. L., Blakeslee, J. P., Ajhar, E. A., \& Dressler, A. 1997, ApJ, 475, in press

Zwicky, F. 1968, Catalogue of Galaxies and of Clusters of Galaxies (Pasadena, CA:

California Institute of Technology) 


\section{Chapter 5}

\section{Globular Clusters in Abell Clusters: Properties and Implications}

\subsection{Analysis}

\subsubsection{Point Source Radial Distributions and Backgrounds}

After rejecting extended objects in the DoPhot output, we corrected the point source counts for incompleteness according to the results of the cloned star experiments described in \$4.4.2. The radial distribution of point sources in each field was then fitted to an $r^{1 / 4}$ law plus background model. The primary reason for this procedure was to determine the background contamination from unresolved galaxies or faint stars. Many different binnings were explored for each field, with the number of bins ranging from as little as 10 to as many as 40 , and an average background value was chosen from among the fits. When the innermost points significantly changed the fit (due to a leveling off of the counts at small radii), they were excluded (similar to the power spectrum fits in $\S 4.4 .3$ ).

Table 5.1 summarizes both the point source and fluctuation measurements, before and after background corrections. For each annular region of each galaxy, the table lists: bright cutoff magnitude $m_{b}$ of the point source counts; corrected number of point sources $N_{p s}\left(\operatorname{arcmin}^{-2}\right)$ fainter than $m_{b}$ but brighter than $m_{c}$; corrected number of GCs $N_{G C}\left(\operatorname{arcmin}^{-2}\right)$ over the same magnitude range following background subtraction; faint end cutoff magnitude $m_{c}$; fitted fluctuation power $P_{0}$ from objects fainter than 
Table 5.1: Point Source Counts and Variance Measurements (Runs 0794 \& 1194.)

\begin{tabular}{rrrrrrcrrrr}
\hline \hline Galaxy.reg & $m_{b}$ & $N_{p s}$ & \multicolumn{1}{c}{ \pm} & \multicolumn{1}{c}{$N_{G C}$} & \multicolumn{1}{c}{ \pm} & $m_{c}$ & \multicolumn{1}{c}{$P_{0}$} & \pm & $P_{G C}$ & \pm \\
\hline A262-1.c1 & 21.0 & 97.6 & 28.1 & 76.6 & 28.3 & 24.0 & 1090 & 53 & 931 & 75 \\
A262-1.c2 & 21.0 & 76.3 & 10.1 & 55.3 & 10.8 & 24.0 & 546 & 24 & 415 & 57 \\
A262-1.c3 & 21.0 & 50.9 & 4.6 & 29.9 & 5.9 & 24.0 & 371 & 15 & 253 & 53 \\
A262-1.c4 & 21.0 & 27.2 & 1.8 & 6.2 & 4.1 & 24.0 & 201 & 18 & 88 & 54 \\
A347-1.c1 & 21.0 & 122.8 & 26.3 & 102.0 & 26.4 & 24.0 & 688 & 39 & 606 & 43 \\
A347-1.c2 & 21.0 & 54.9 & 8.4 & 34.1 & 8.6 & 24.0 & 313 & 17 & 243 & 24 \\
A347-1.c3 & 21.0 & 30.5 & 3.2 & 9.7 & 3.7 & 24.0 & 189 & 4 & 123 & 17 \\
A347-1.c4 & 21.0 & 22.5 & 1.4 & 1.7 & 2.3 & 24.0 & 103 & 7 & 39 & 18 \\
A2199-1.c2 & 21.5 & 100.0 & 15.6 & 74.0 & 15.7 & 24.0 & 311 & 29 & 262 & 31 \\
A2199-1.c3 & 21.5 & 68.9 & 6.0 & 42.9 & 6.2 & 24.0 & 151 & 8 & 107 & 12 \\
A2199-1.c4 & 21.5 & 36.1 & 2.1 & 10.1 & 2.6 & 24.0 & 89 & 6 & 48 & 11 \\
A2666-1.c1 & 21.5 & 94.5 & 38.8 & 76.1 & 38.9 & 24.5 & 848 & 115 & 712 & 121 \\
A2666-1.c2 & 21.5 & 27.7 & 6.8 & 9.3 & 7.4 & 24.5 & 412 & 23 & 297 & 43 \\
A2666-1.c3 & 21.5 & 57.1 & 5.1 & 24.8 & 7.6 & 25.0 & 128 & 13 & 59 & 26 \\
A2666-1.c4 & 21.5 & 39.9 & 2.4 & 7.6 & 6.1 & 25.0 & 94 & 7 & 28 & 24 \\
\hline \hline
\end{tabular}

$m_{c}$, in units of $10^{3}\left(e^{-} / \text {pixel }\right)^{2}$; background-subtracted power $P_{G C}$ due to GCs fainter than $m_{c}$, also in $10^{3}\left(e^{-} / \text {pixel }\right)^{2}$. The following section describes how $P_{G C}$ is obtained from $P_{0}$; here we discuss the point sources in more detail.

Figures 5-1 through 5-21 display the radial distributions of point sources around each galaxy and the fitted $r^{1 / 4}$ law plus background model. The fits and distributions are shown for the magnitude range $m_{b}-m_{c}$ listed in Table 5.1 for the $c 4$ region of each galaxy. For these figures, $\sim 15$ bins were used, slightly more when the number of sources was large, a few less when the field was relatively sparse. The errorbars are dominated by Poisson statistics but include contributions from the small completeness corrections applied.

The dashed horizontal line is drawn at the fitted background level, while the dotted line just above it represents the unbiased background estimator proposed by Harris (1986), who found that the backgrounds estimated from fitting $r^{1 / 4}$ laws to GC distributions tended to be too low. This finding was based on experiments in fitting the radial GC distributions of Virgo and other nearby elliptical galaxies when the true background was already known from images offset from the galaxy, and the best 
Table 5.1-Continued. (Run 0295.)

\begin{tabular}{|c|c|c|c|c|c|c|c|c|c|c|}
\hline Galaxy.reg & $m_{b}$ & $N_{p s}$ & \pm & $N_{G C}$ & \pm & $m_{c}$ & $P_{0}$ & \pm & $P_{G C}$ & \pm \\
\hline A539-1.c1 & 22.0 & 80.8 & 24.4 & 63.1 & 24.7 & 24.5 & 1813 & 214 & 1539 & 233 \\
\hline A539-1.c2 & 22.0 & 44.7 & 7.8 & 27.0 & 8.6 & 24.5 & 775 & 43 & 517 & 101 \\
\hline A539-1.c3 & 22.0 & 35.0 & 4.0 & 17.3 & 5.4 & 24.5 & 520 & 13 & 267 & 93 \\
\hline A539-1.c4 & 22.0 & 22.3 & 1.5 & 4.6 & 3.9 & 24.5 & 285 & 15 & 34 & 93 \\
\hline A539-2.c1 & 22.0 & 101.8 & 31.1 & 80.2 & 31.2 & 24.5 & 2217 & 76 & 1954 & 118 \\
\hline A539-2.c2 & 22.0 & 92.5 & 13.5 & 70.9 & 13.7 & 24.5 & 1055 & 26 & 802 & 94 \\
\hline A539-2.c3 & 22.0 & 44.9 & 4.6 & 23.3 & 5.2 & 24.5 & 562 & 18 & 315 & 92 \\
\hline A539-2.c4 & 22.0 & 24.1 & 1.7 & 2.5 & 3.0 & 24.5 & 326 & 17 & 82 & 92 \\
\hline A569-1.c1 & 21.5 & 16.1 & 9.4 & 4.7 & 9.6 & 24.0 & 336 & 31 & 260 & 38 \\
\hline A569-1.c2 & 21.5 & 68.2 & 11.0 & 45.1 & 11.2 & 24.5 & 94 & 7 & 53 & 15 \\
\hline A569-1.c3 & 21.5 & 35.3 & 3.6 & 12.2 & 4.3 & 24.5 & 49 & 2 & 12 & 13 \\
\hline A569-1.c4 & 21.5 & 24.5 & 1.6 & 1.4 & 2.8 & 24.5 & 44 & 2 & 8 & 13 \\
\hline A634-1.c1 & 22.0 & 145.8 & 32.4 & 128.0 & 32.4 & 24.5 & 2282 & 118 & 1807 & 145 \\
\hline A634-1.c2 & 22.0 & 48.1 & 9.1 & 30.3 & 9.2 & 24.5 & 1023 & 62 & 591 & 104 \\
\hline A634-1.c3 & 22.0 & 50.3 & 4.2 & 13.7 & 4.6 & 25.0 & 318 & 10 & 54 & 53 \\
\hline A634-1.c4 & 22.0 & 38.4 & 2.0 & 1.8 & 2.7 & 25.0 & 237 & 13 & -25 & 54 \\
\hline A $779-1 . c 1$ & 21.5 & 86.6 & 36.2 & 68.1 & 36.4 & 24.5 & 1083 & 59 & 940 & 65 \\
\hline A779-1.c2 & 21.5 & 113.7 & 14.8 & 78.2 & 15.6 & 25.0 & 309 & 26 & 227 & 31 \\
\hline A779-1.c3 & 21.5 & 91.2 & 6.3 & 55.7 & 7.9 & 25.0 & 188 & 7 & 118 & 16 \\
\hline A779-1.c4 & 21.5 & 45.3 & 2.2 & 9.8 & 5.3 & 25.0 & 112 & 8 & 47 & 16 \\
\hline A999-1.c1 & 22.0 & 98.5 & 36.6 & 68.2 & 36.6 & 25.0 & 1068 & 46 & 775 & 81 \\
\hline A999-1.c2 & 22.0 & 60.1 & 10.6 & 29.8 & 10.7 & 25.0 & 512 & 31 & 228 & 74 \\
\hline A999-1.c3 & 22.0 & 35.7 & 4.2 & 5.4 & 4.5 & 25.0 & 345 & 25 & 67 & 71 \\
\hline A999-1.c4 & 22.0 & 54.6 & 2.3 & 3.9 & 3.8 & 25.5 & 133 & 8 & -40 & 42 \\
\hline A1016-1.c1 & 22.0 & 119.2 & 30.9 & 92.5 & 30.9 & 25.0 & 685 & 29 & 451 & 58 \\
\hline A1016-1.c2 & 22.0 & 88.4 & 12.1 & 42.6 & 12.4 & 25.5 & 226 & 11 & 84 & 33 \\
\hline A1016-1.c3 & 22.0 & 53.1 & 4.9 & 7.3 & 5.7 & 25.5 & 153 & 5 & 15 & 31 \\
\hline A1016-1.c4 & 22.0 & 49.8 & 2.5 & 4.0 & 3.8 & 25.5 & 111 & 4 & -26 & 31 \\
\hline A1177-1.c2 & 22.0 & 34.1 & 8.3 & 18.5 & 8.5 & 24.5 & 1796 & 94 & 1158 & 150 \\
\hline A1177-1.c3 & 22.0 & 86.4 & 5.9 & 30.9 & 6.3 & 25.5 & 384 & 7 & 137 & 46 \\
\hline A1177-1.c4 & 22.0 & 58.3 & 2.3 & 2.8 & 3.2 & 25.5 & 251 & 13 & 8 & 47 \\
\hline A1367-1.c1 & 21.5 & 201.3 & 38.3 & 185.6 & 38.3 & 24.5 & 448 & 32 & 396 & 35 \\
\hline A1367-1.c2 & 21.5 & 103.5 & 11.9 & 87.8 & 12.0 & 24.5 & 236 & 8 & 194 & 15 \\
\hline A1367-1.c3 & 21.5 & 76.3 & 5.2 & 43.3 & 6.0 & 25.0 & 64 & 4 & 40 & 9 \\
\hline A1367-1.c4 & 21.5 & 47.9 & 2.2 & 14.9 & 3.7 & 25.0 & 38 & 1 & 16 & 0 \\
\hline
\end{tabular}


Table 5.1-Continued. (Runs 0395 \& 0995.)

\begin{tabular}{|c|c|c|c|c|c|c|c|c|c|c|}
\hline Galaxy.reg & $m_{b}$ & $N_{p s}$ & \pm & $N_{G C}$ & \pm & $m_{c}$ & $\overline{P_{0}}$ & \pm & $P_{G C}$ & \pm \\
\hline A347-1.c1 & 21.5 & 226.0 & 36.5 & 199.3 & 36.7 & 24.5 & 302 & 21 & 261 & 22 \\
\hline A347-1.c2 & 21.5 & 104.3 & 11.7 & 77.6 & 12.4 & 24.5 & 120 & 7 & 88 & 10 \\
\hline A347-1.c3 & 21.5 & 62.5 & 4.7 & 35.8 & 6.2 & 24.5 & 69 & 1 & 40 & 7 \\
\hline A347-1.c4 & 21.5 & 36.8 & 1.9 & 10.1 & 4.5 & 24.5 & 41 & 2 & 13 & 7 \\
\hline A397-1.c1 & 22.0 & 100.1 & 26.0 & 77.5 & 26.1 & 25.0 & 1549 & 71 & 1288 & 87 \\
\hline A397-1.c2 & 22.0 & 61.3 & 9.7 & 38.7 & 9.9 & 25.0 & 886 & 74 & 653 & 88 \\
\hline A397-1.c3 & 22.0 & 29.2 & 3.3 & 6.6 & 3.8 & 25.0 & 548 & 23 & 324 & 53 \\
\hline A397 & 22.0 & 24.0 & 1.4 & 1.4 & 2.3 & 25.0 & 331 & 16 & 111 & 50 \\
\hline A569 & 21.5 & 26.4 & 13.9 & 9.5 & 14.1 & 24.0 & 621 & 97 & 485 & 105 \\
\hline A569-1.c2 & 21.5 & 35.4 & 7.1 & 18.5 & 7.5 & 24.0 & 317 & 22 & 202 & 44 \\
\hline A569-1.c3 & 21.5 & 21.6 & 2.7 & 4.7 & 3.6 & 24. & 175 & 20 & 68 & 42 \\
\hline A569-1.c4 & 21.5 & 17.6 & 1.4 & 0.7 & 2.8 & 24 & 150 & 16 & 45 & 41 \\
\hline A1185-1.c1 & 22.0 & 126.8 & 51.2 & 99.0 & 51.3 & 25.0 & 5627 & 44 & 921 & 367 \\
\hline A1185-1.c2 & 22.0 & 62.1 & 9.9 & 34.3 & 10.2 & 25.0 & 2327 & 137 & 1683 & 184 \\
\hline A1185-1.c3 & 22.0 & 39.6 & 3.8 & 11.8 & 4.6 & 25.0 & 1377 & 33 & 755 & 127 \\
\hline A1185-1.c4 & 22.0 & 30.1 & 1.9 & 2.3 & 3.2 & 25.0 & 889 & 54 & 274 & 134 \\
\hline A1314-1.c1 & 22.0 & 84.6 & 28.8 & 57.1 & 28.9 & 25.0 & 2531 & 239 & 1898 & 263 \\
\hline $\mathrm{A} 1314$ & 22.0 & 85.9 & 12.3 & 58.4 & 12.6 & 25.0 & 1439 & 123 & 838 & 164 \\
\hline A131 & 22.0 & 51.6 & 4.8 & 24.1 & 5.6 & 25.0 & 908 & 34 & 323 & 113 \\
\hline A1314-1.c4 & 21.5 & 64.6 & 3.0 & 20.4 & 6.2 & 25.5 & 342 & 12 & -19 & 68 \\
\hline A1656-3.c1 & 21.5 & 152.5 & 30.4 & 127.5 & 30.5 & 24.5 & 2417 & 103 & 2099 & 136 \\
\hline A1656-3.c2 & 21.5 & 62.9 & 9.2 & 37.9 & 9.5 & 24 & 1323 & 96 & 1053 & 128 \\
\hline A1656-3.c3 & 21.5 & 36.1 & 3.4 & 11.1 & 4.2 & 24.5 & 600 & 22 & 347 & 88 \\
\hline A1656-3.c4 & 21.5 & 27.5 & 1.7 & 2.5 & 3.0 & 24.5 & 391 & 21 & 144 & 88 \\
\hline A2197-1.c2 & 22.0 & 39.3 & 8.0 & 21.0 & 9.0 & 24.5 & 2511 & 189 & 1406 & 430 \\
\hline A2197-1.c3 & 22.0 & 25.3 & 3.1 & 7.0 & 5.2 & 24.5 & 1318 & 126 & 252 & 406 \\
\hline A2197-1.c4 & 22.0 & 21.7 & 1.3 & 3.4 & 4.4 & 24.5 & 1128 & 58 & 73 & 390 \\
\hline A2197-2.c1 & 22.0 & 161.8 & 50.3 & 138.3 & 50.5 & 25.0 & 1451 & 61 & 1221 & 79 \\
\hline A219 & 22.0 & 81.7 & 12.7 & 58.2 & 13.6 & 25.0 & 857 & 56 & 653 & 74 \\
\hline c3 & 22.0 & 60.0 & 5.0 & 36.5 & 7.0 & 25.0 & 499 & 15 & 307 & 51 \\
\hline A219? & 22.0 & 32.0 & 1.7 & 8.5 & 5.2 & 25.0 & 281 & 14 & 94 & 51 \\
\hline A2162-1.c1 & 22.0 & 55.7 & 20.1 & 45.1 & 20.2 & 24.0 & 5886 & 539 & 5009 & 569 \\
\hline A2162-1.c2 & 22.0 & 71.3 & 12.1 & 50.9 & 12.3 & 24 . & 1775 & 124 & 1240 & 168 \\
\hline A2162-1.c3 & 22.0 & 37.4 & 3.8 & 17.0 & 4.4 & 24 & 83 & 40 & 312 & 120 \\
\hline A2162-1.c4 & 22.0 & 23.7 & 1.6 & 3.3 & 2.7 & 24.5 & 616 & 34 & 96 & 118 \\
\hline A2634-1.c1 & 22.0 & 110.3 & 30.4 & 91.5 & 30.7 & 24.5 & 2435 & 158 & 2162 & 173 \\
\hline A2634-1.c2 & 22.0 & 75.6 & 10.5 & 56.8 & 11.3 & 24.5 & 1207 & 78 & 960 & 104 \\
\hline A2634-1.c3 & 22.0 & 47.2 & 4.2 & 28.4 & 5.9 & 24.5 & 738 & 37 & 501 & 77 \\
\hline A2634-1.c4 & 22.0 & 24.2 & 1.6 & 5.4 & 4.4 & 24.5 & 429 & 13 & 195 & 69 \\
\hline
\end{tabular}




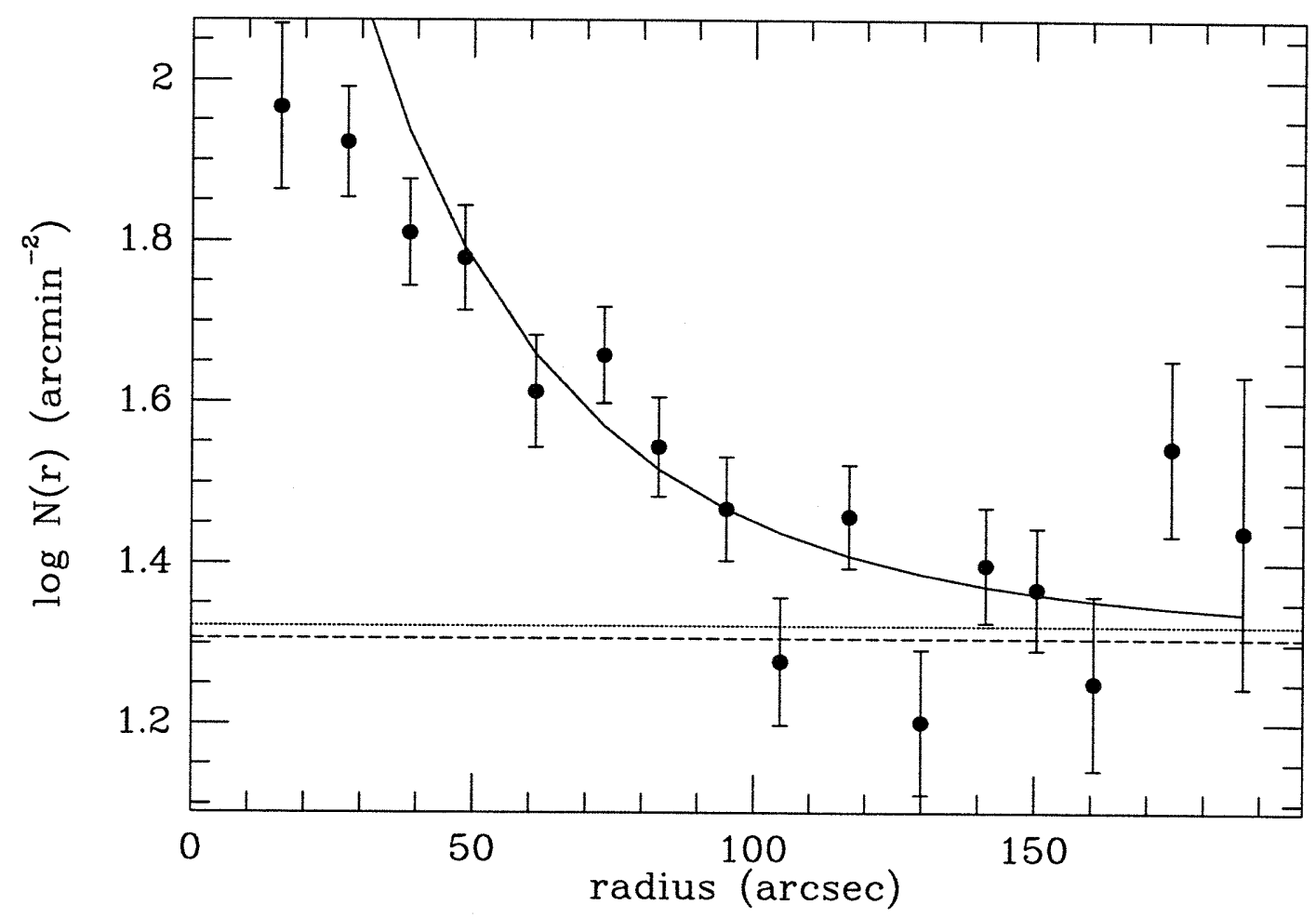

Figure 5-1: A262-1 point source distribution and fit. See text for description.

proposed estimator was the geometric mean of the fitted background and the value of the model at the radial limit of the fit. Harris reported that this quantity gave an unbiased background estimate as long as it differed from the fitted background by $\lesssim 15 \%$, as is the case for all the galaxies here; thus, this estimator was adopted.

Although the primary purpose of the fits was to determine the background contamination, the figures are probably more interesting for the graphical information they supply about the radial distributions. Several of the distributions show an apparent central deficit of GCs compared to the $r^{1 / 4}$ law fit (e.g., A262-1, A539-2, A2197-2) which could be due to an undercorrection for central incompleteness, but the fact that most of the other distributions show no signs of central flattening indicates that we are correcting correctly. Thus, these galaxies probably have larger apparent core radii to their GC distributions, probably due to dynamical evolution, as discussed by Forbes et al. (1996a). In general though, the figures show that distributions follow $r^{1 / 4}$ laws quite well, with the A1367-1 distribution following its fitted $r^{1 / 4}$ law miraculously well. 


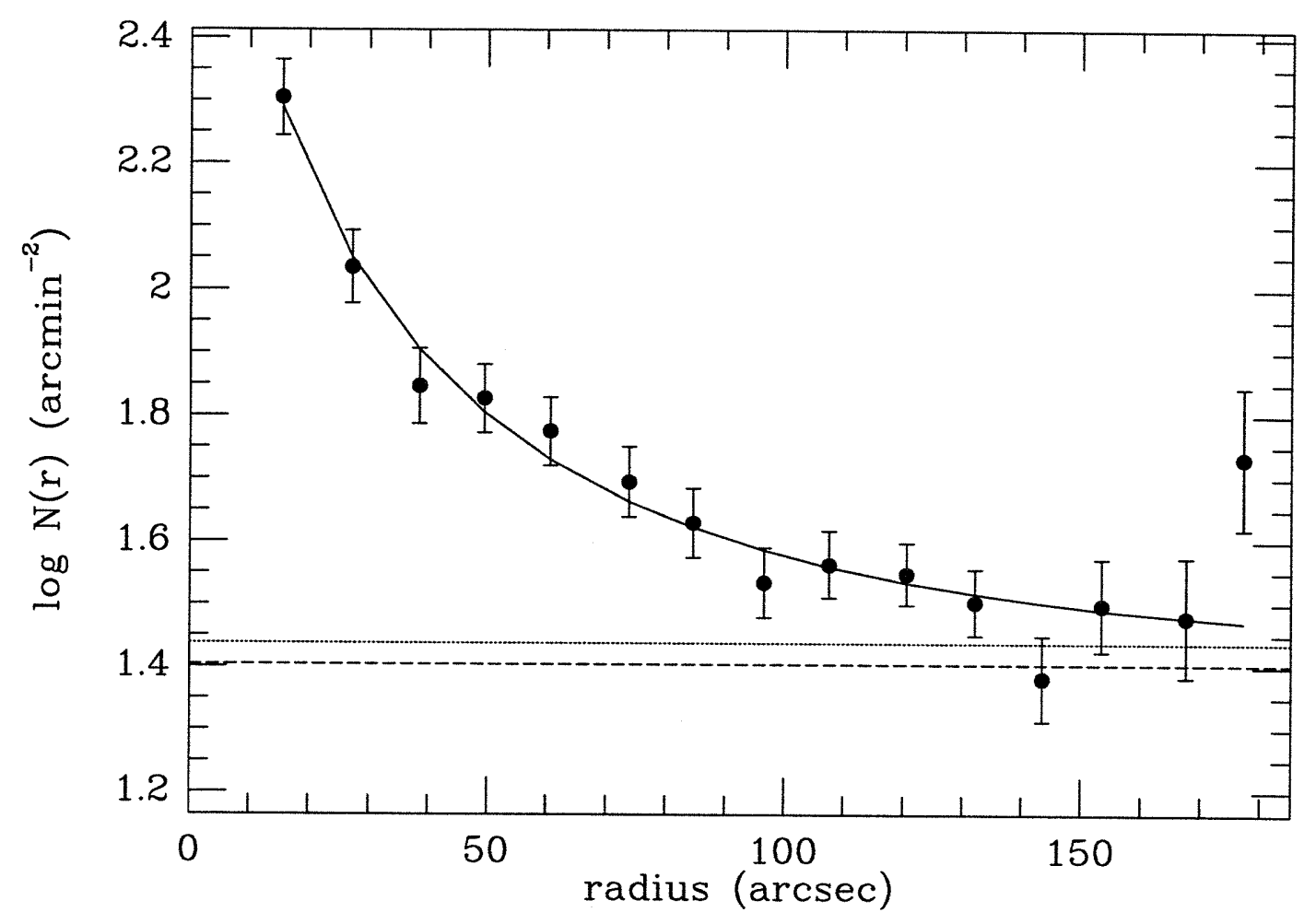

Figure 5-2: A347-1.

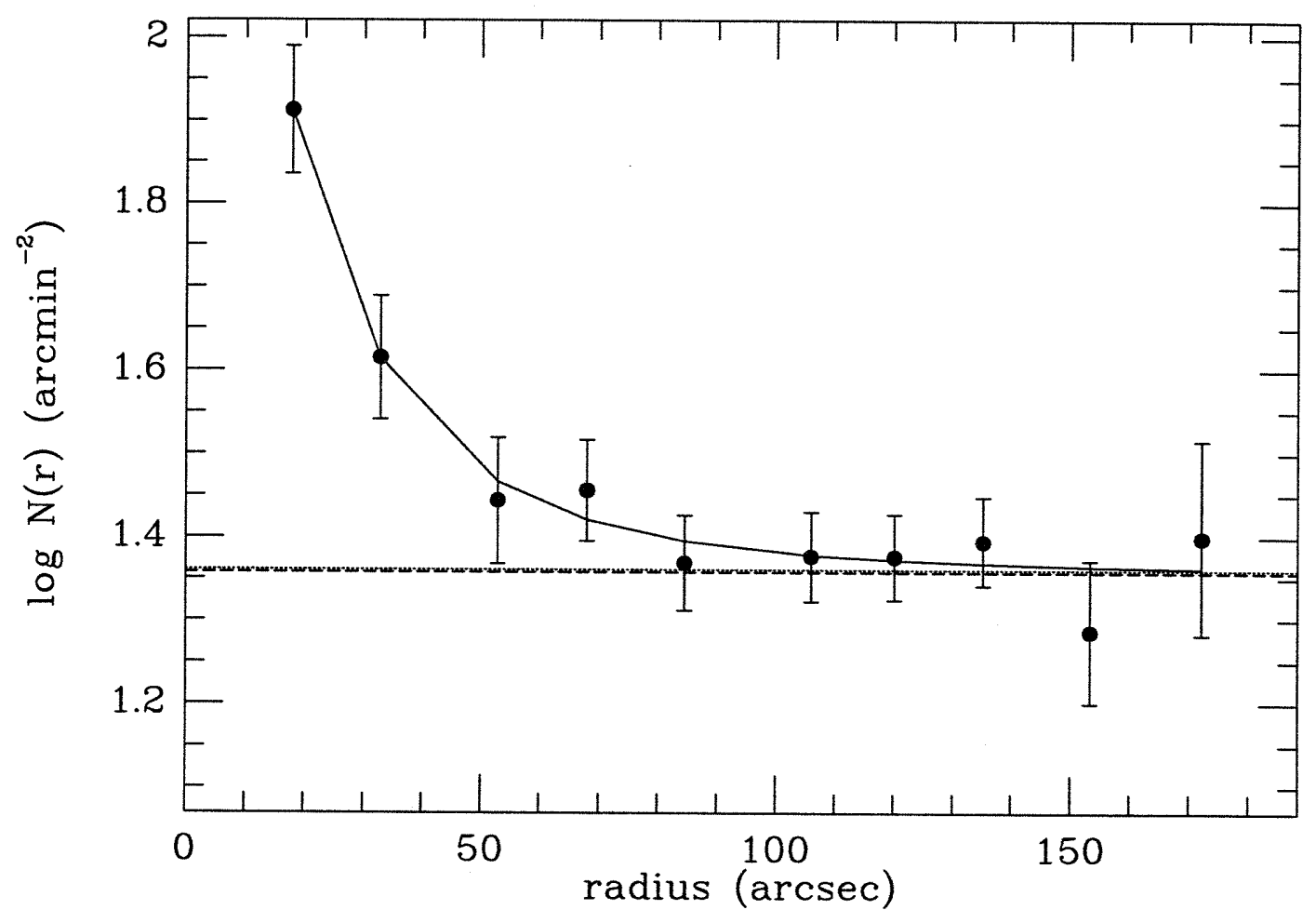

Figure 5-3: A397-1. 


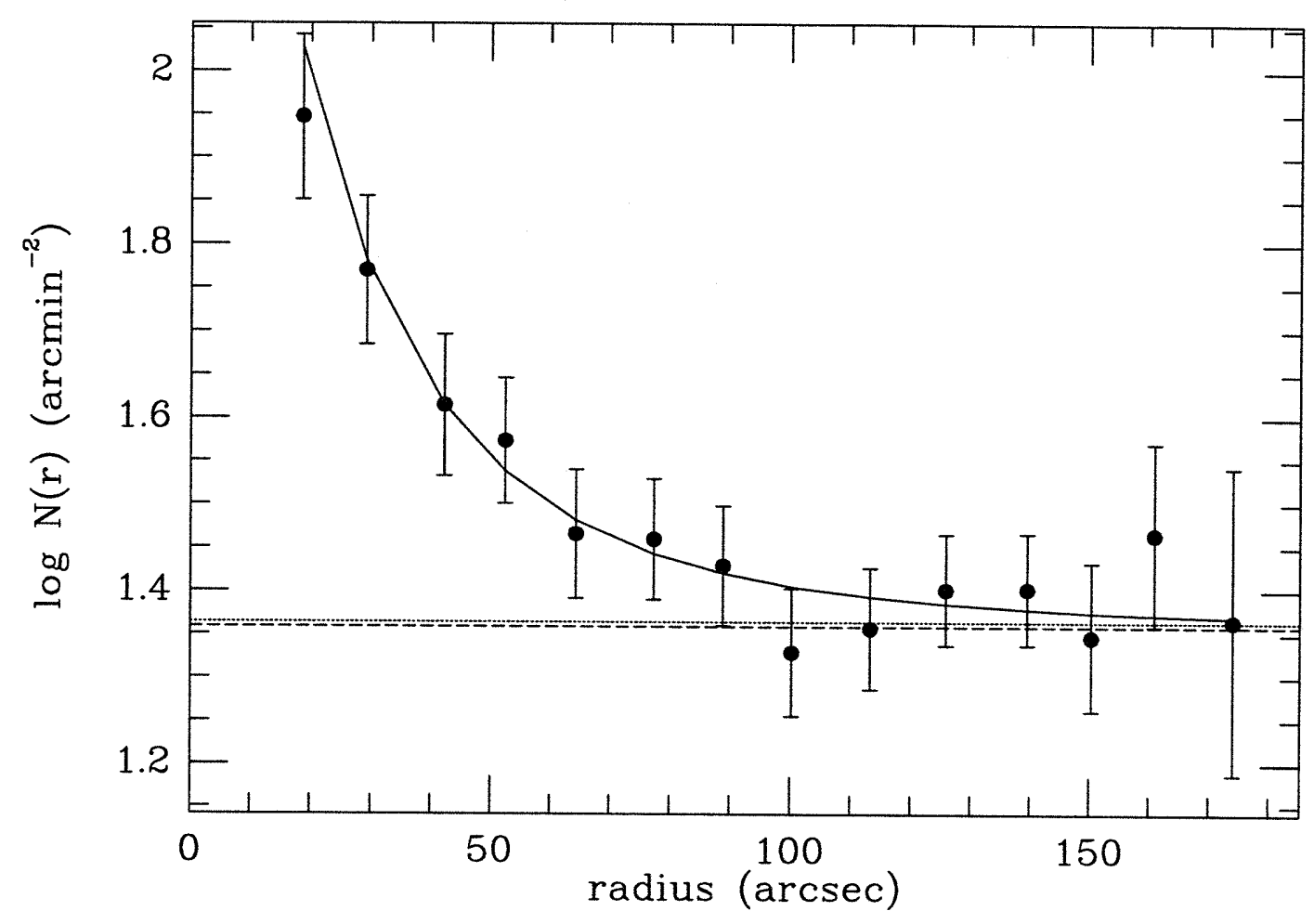

Figure 5-4: A569-1.

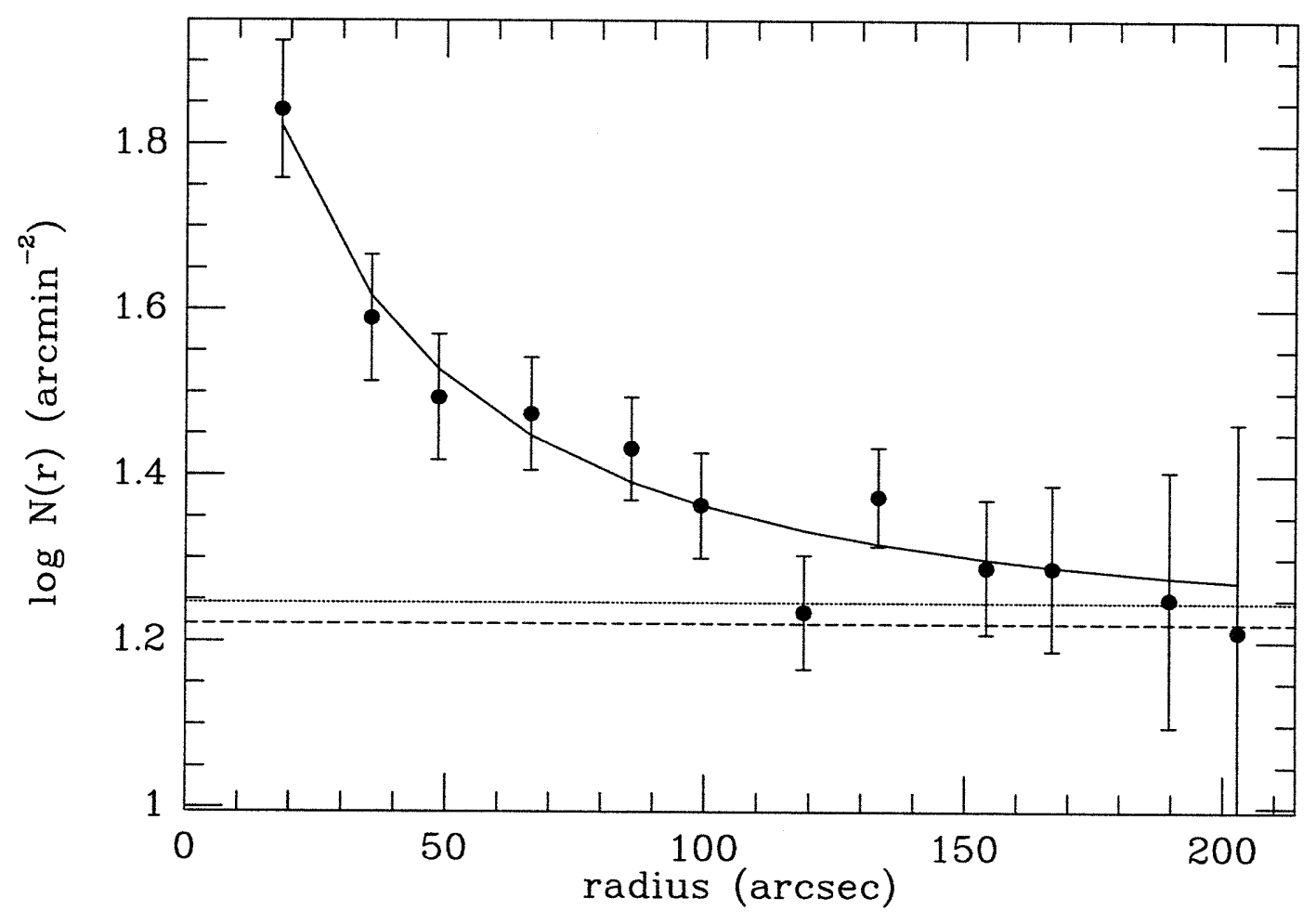

Figure 5-5: A539-1. 


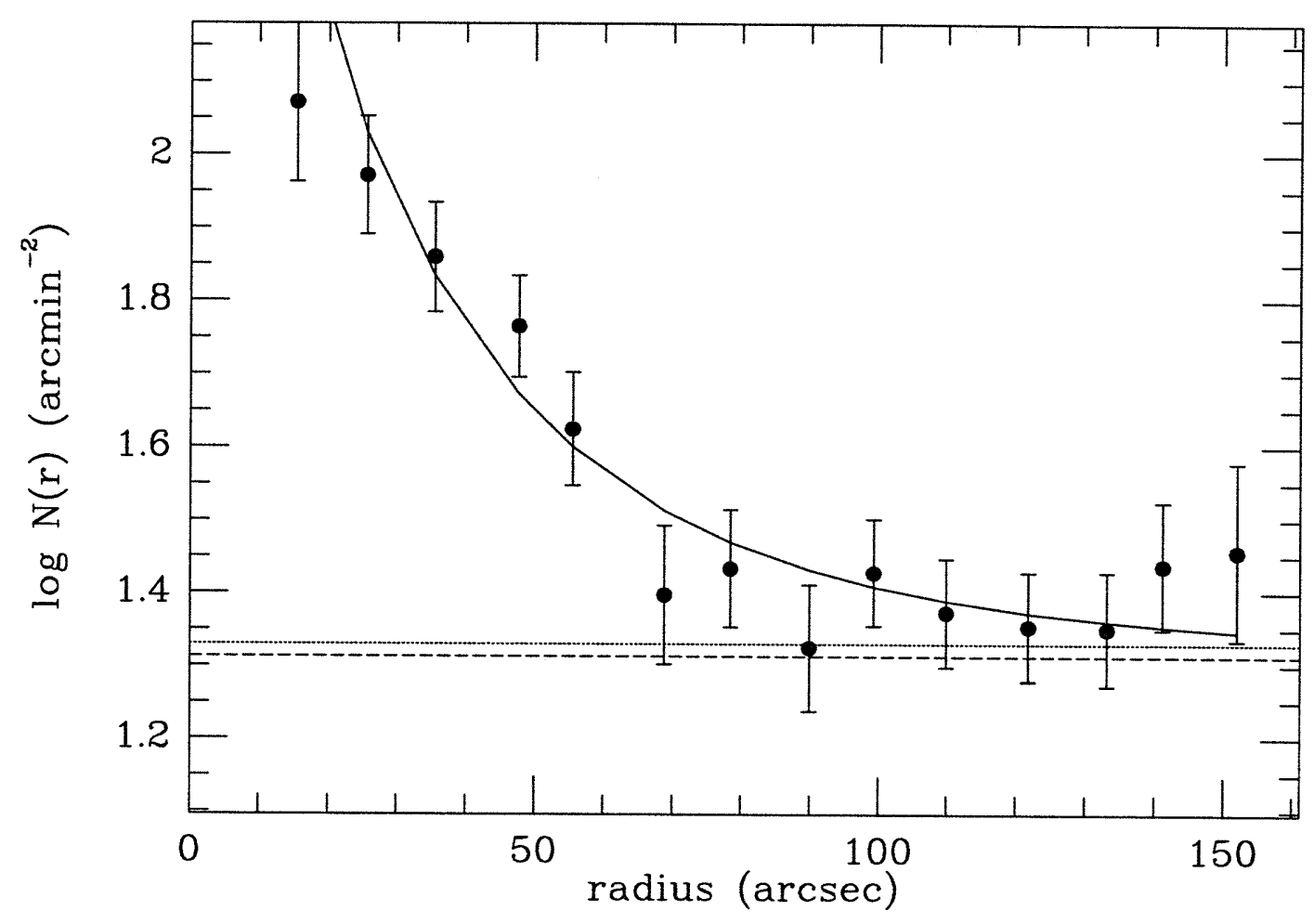

Figure 5-6: A539-2.

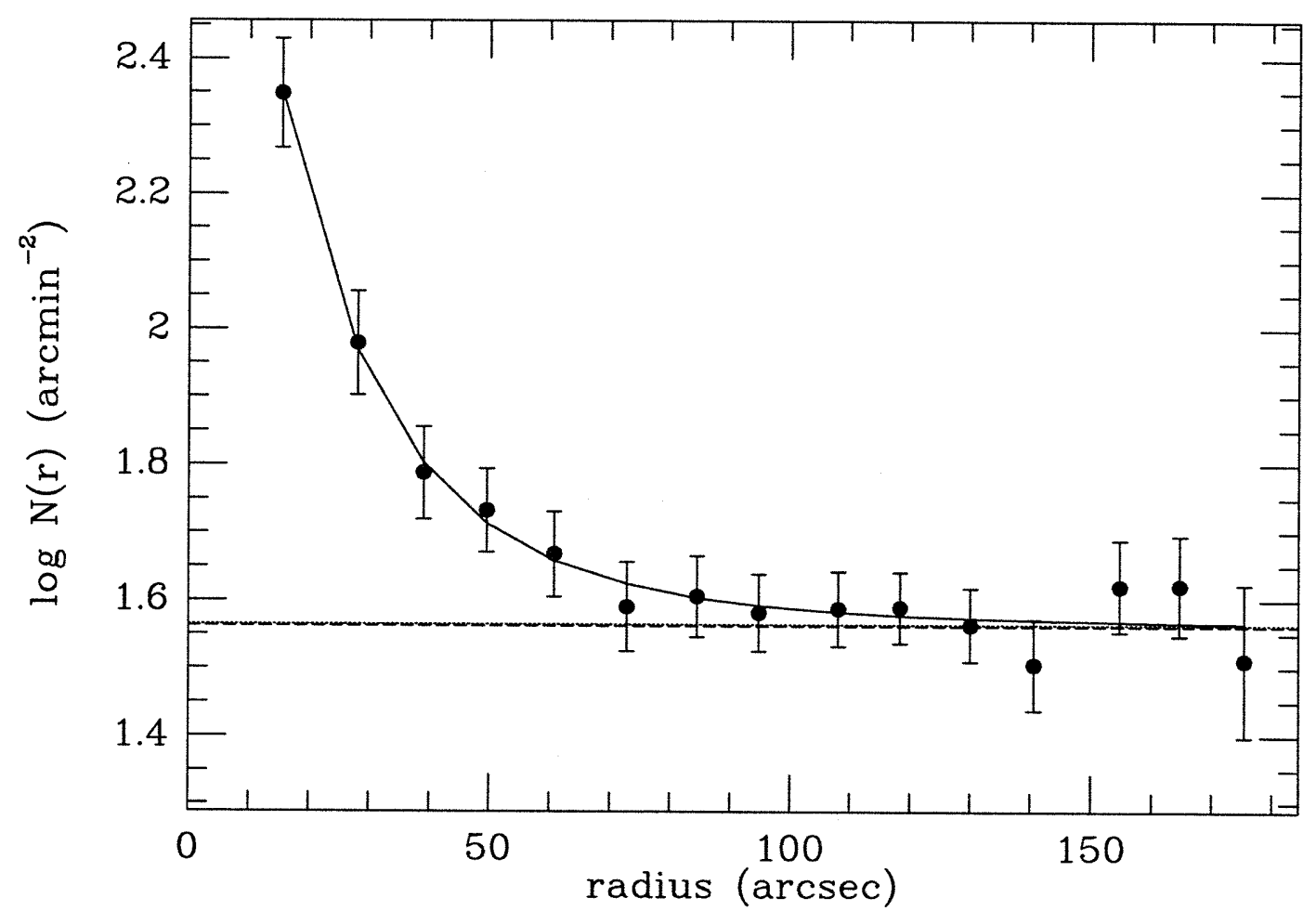

Figure 5-7: A634-1. 


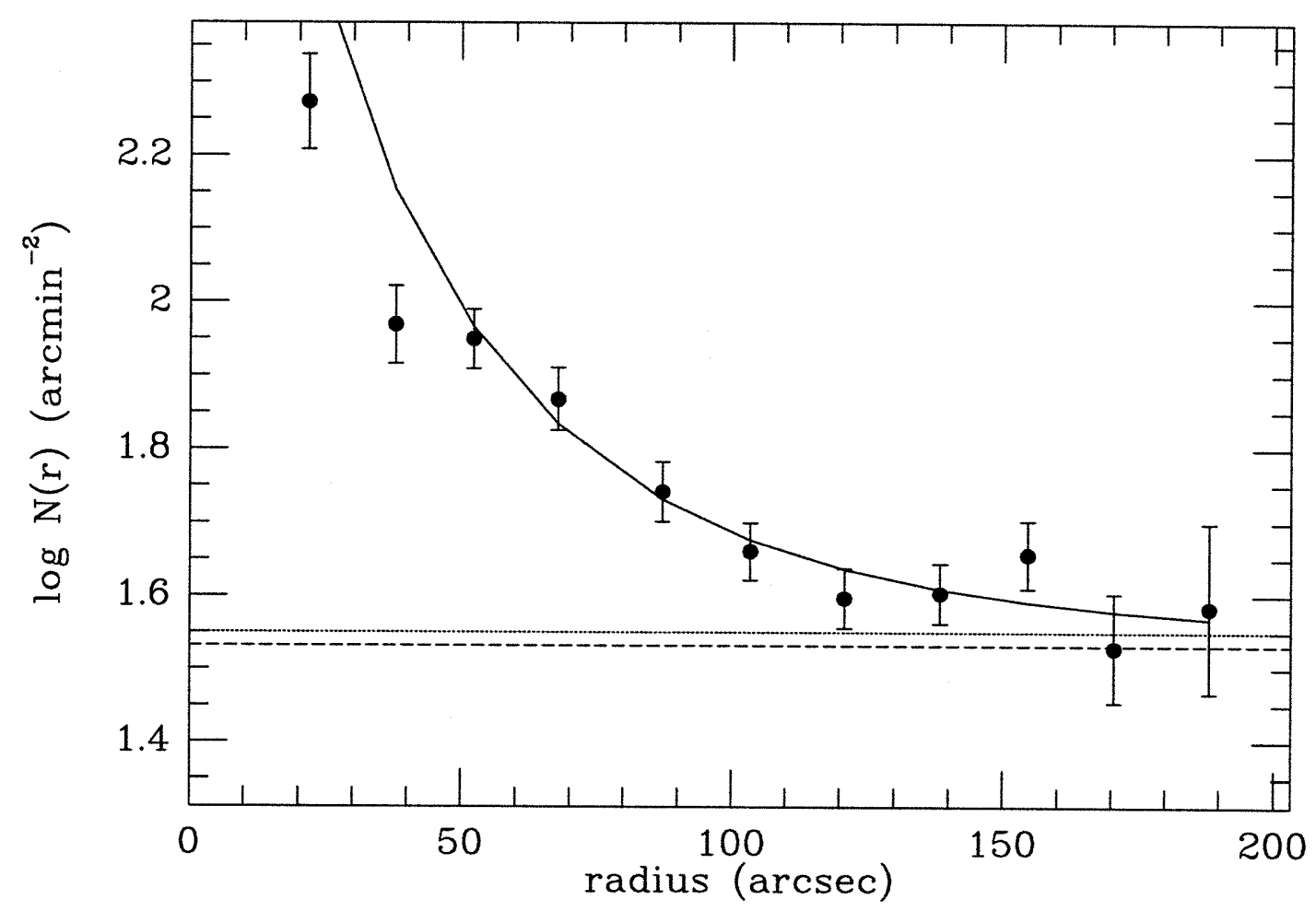

Figure 5-8: A779-1.

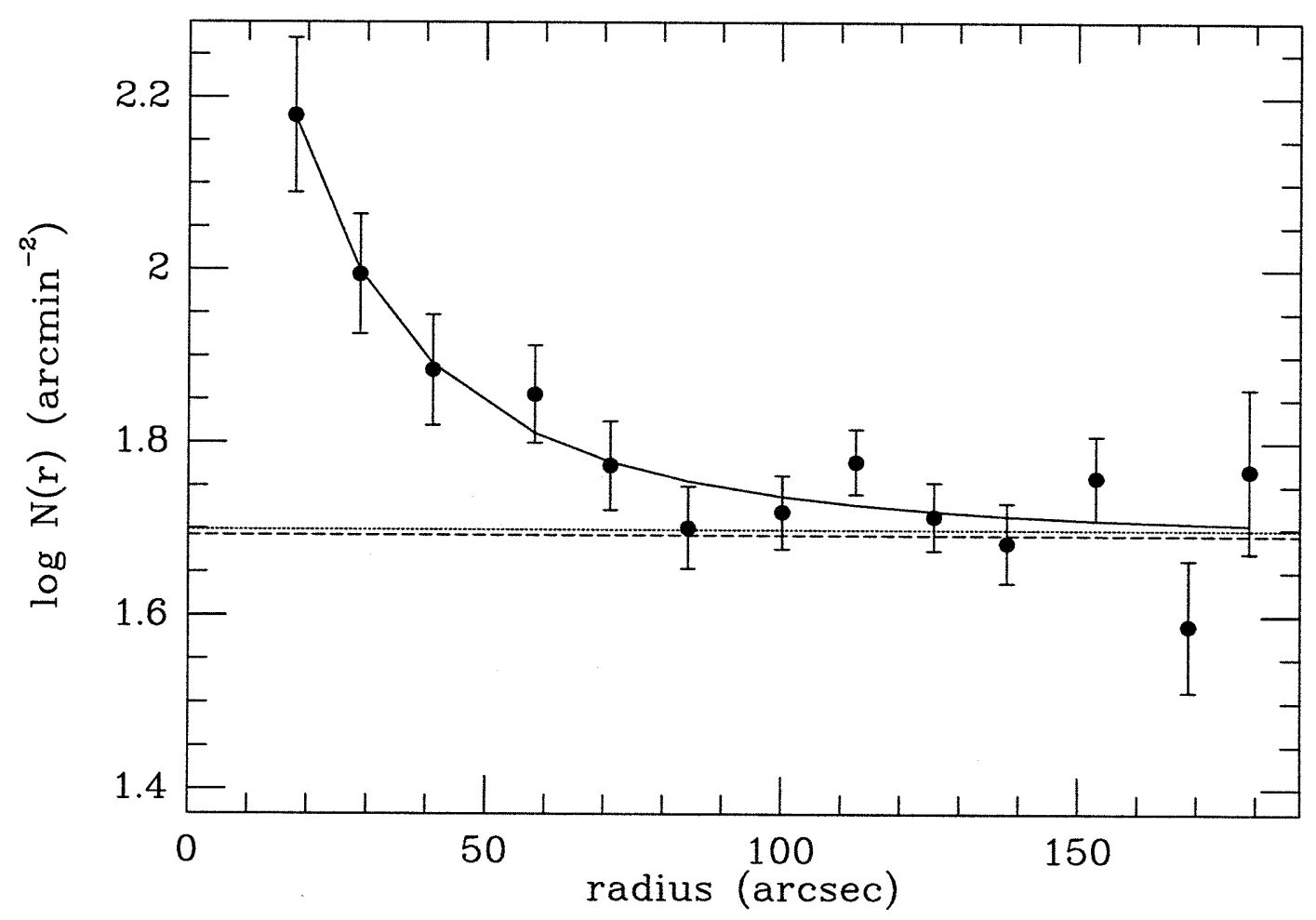

Figure 5-9: A999-1. 


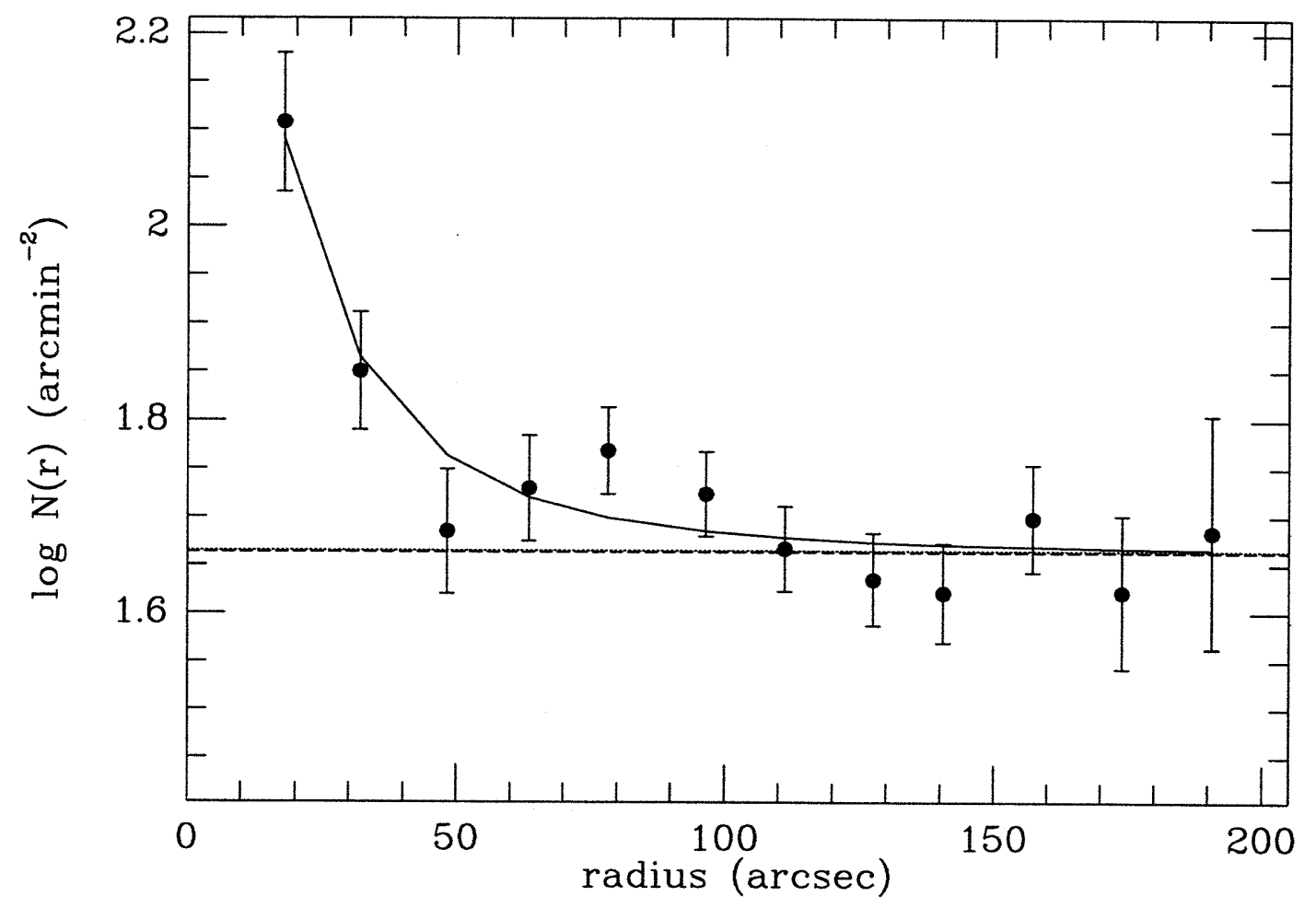

Figure 5-10: A1016-1.

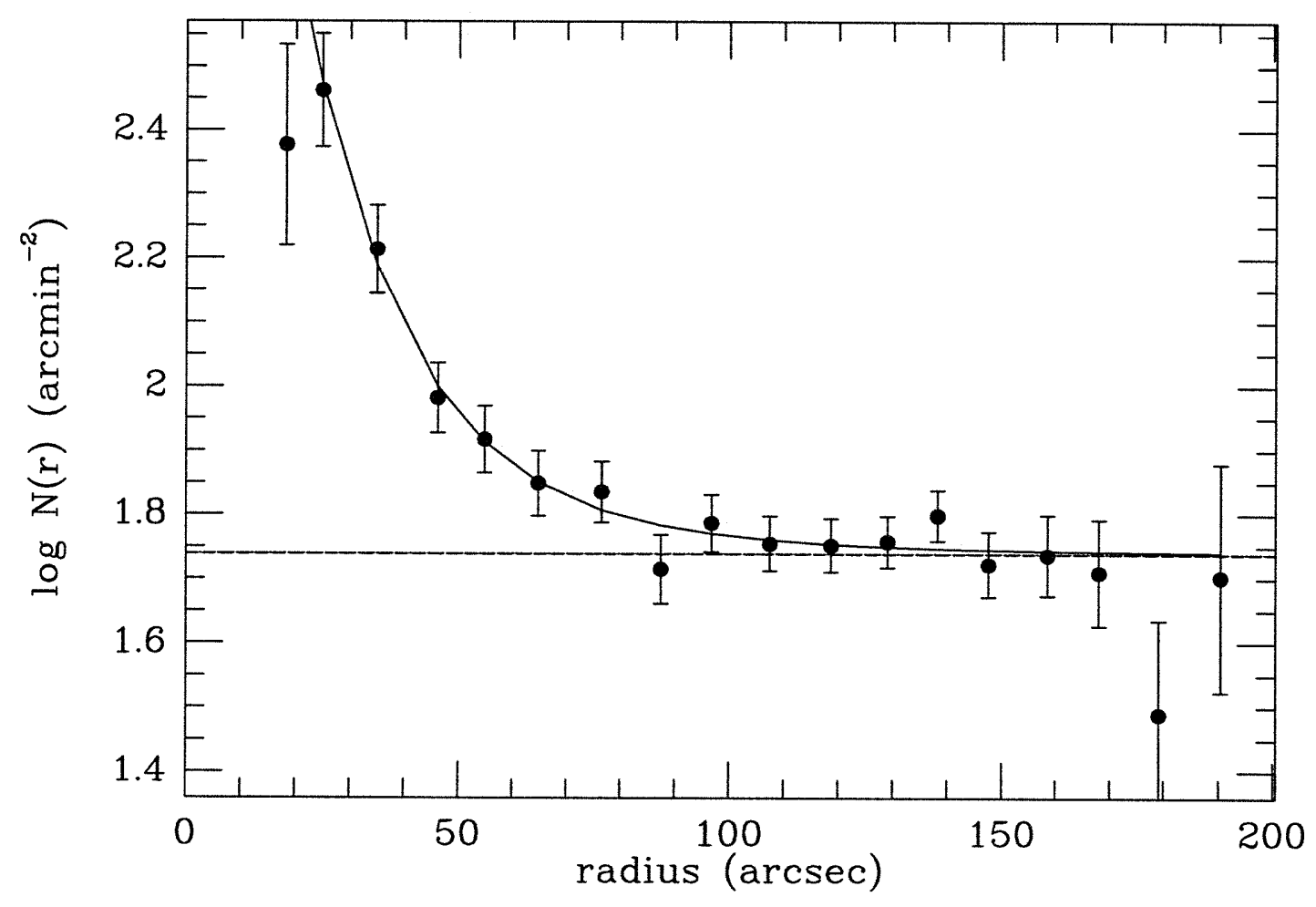

Figure 5-11: A1177-1. 


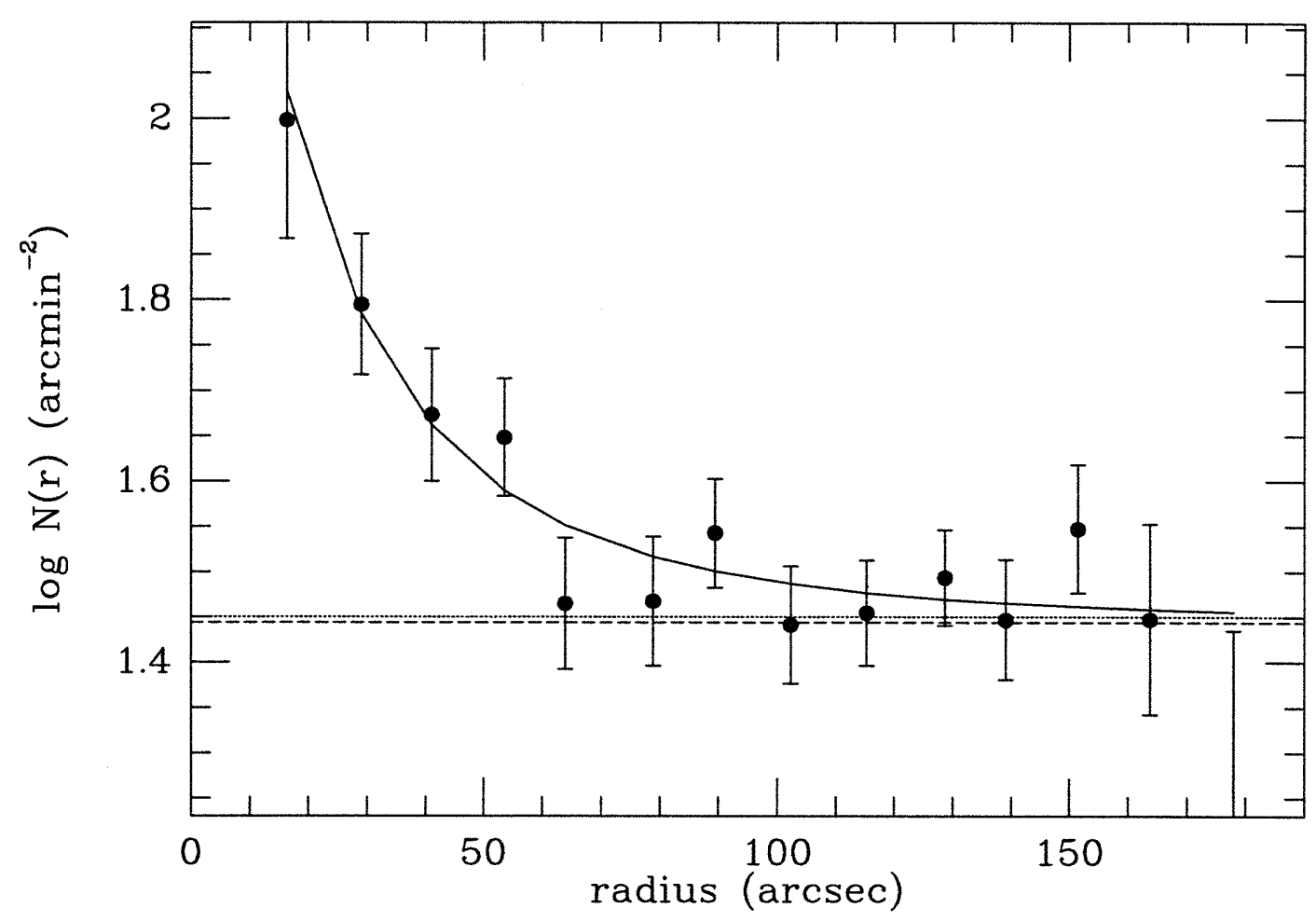

Figure 5-12: A1185-1.

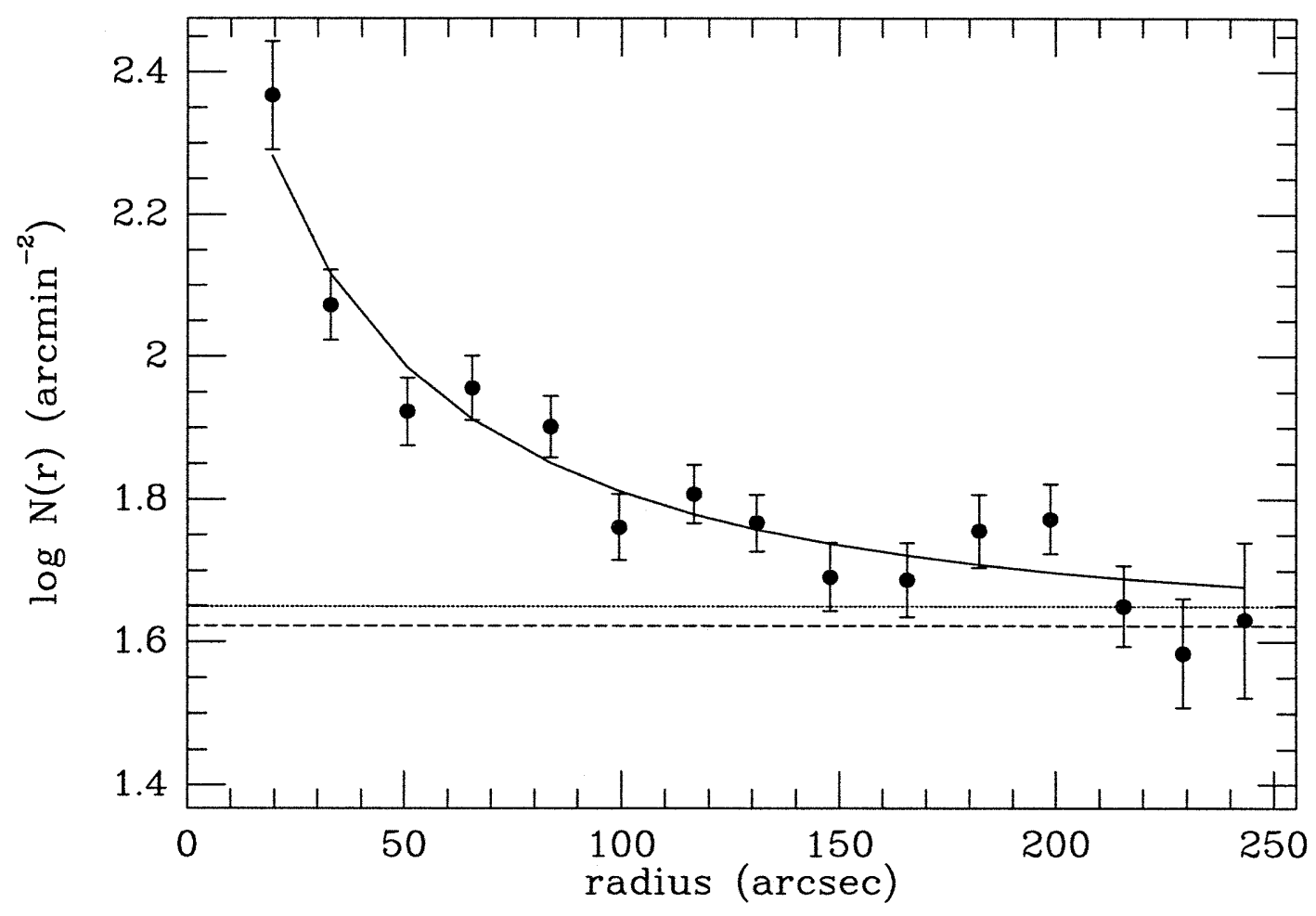

Figure 5-13: A1314-1. 


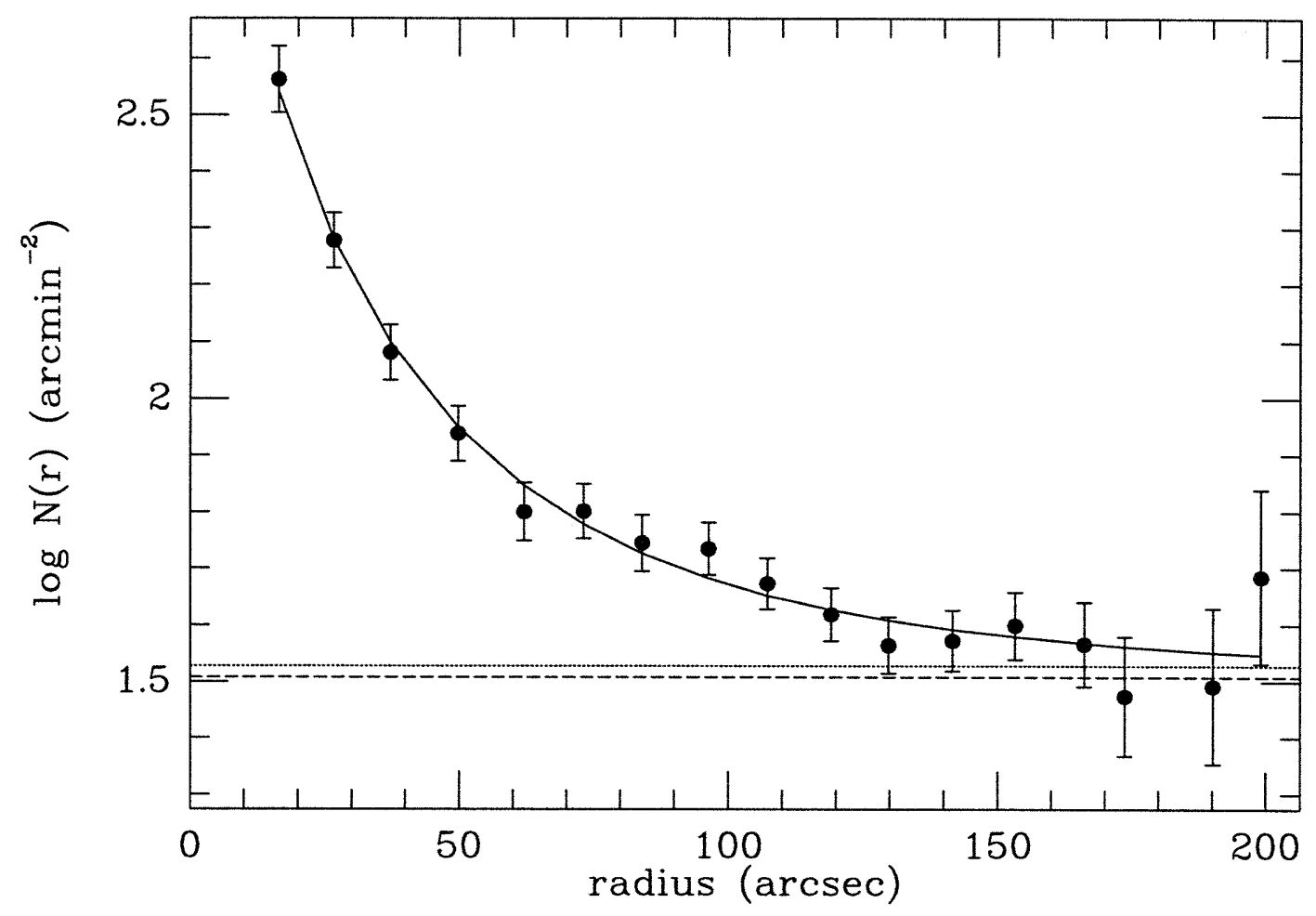

Figure 5-14: A1367-1.

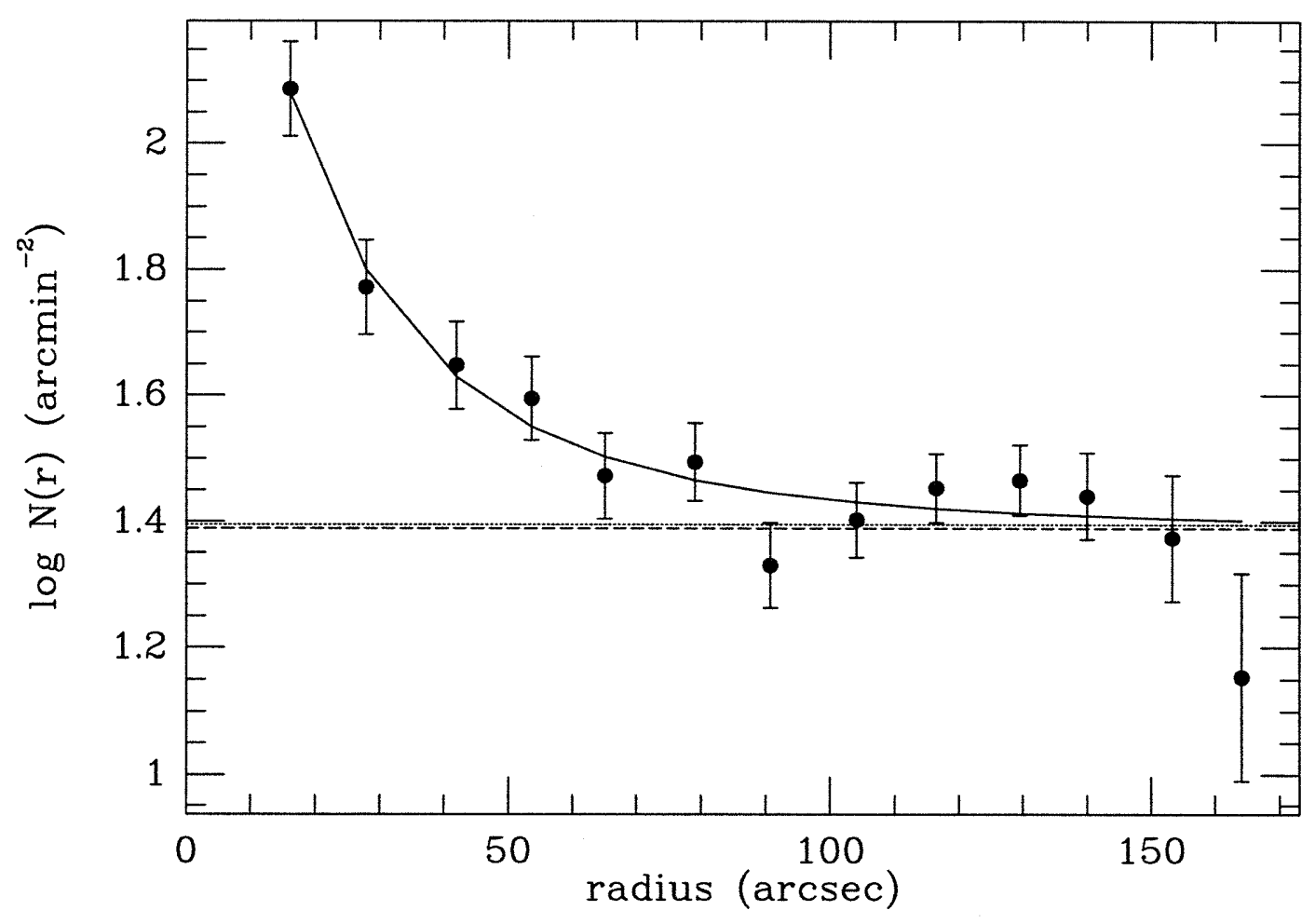

Figure 5-15: A1656-3. 


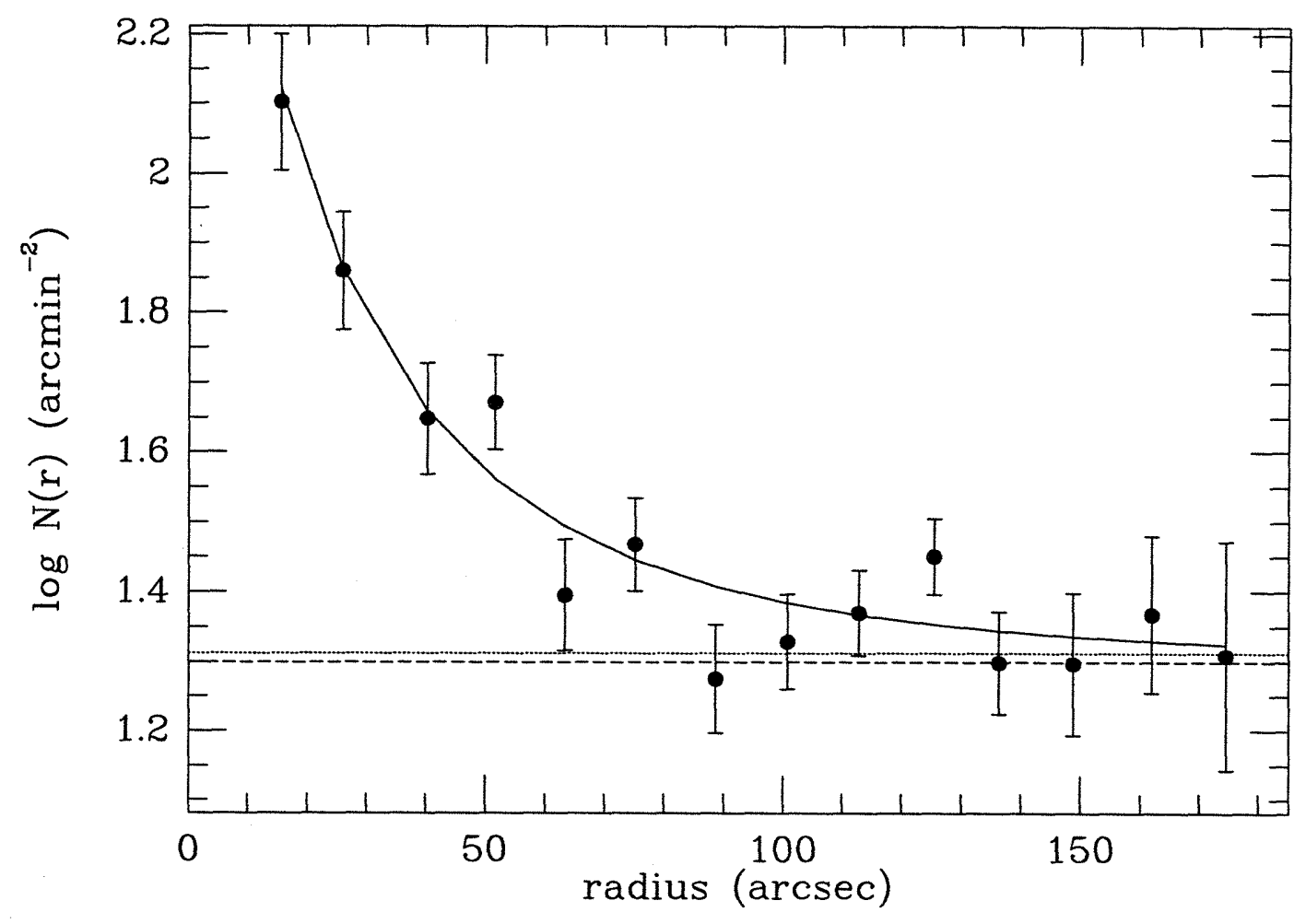

Figure 5-16: A2162-1.

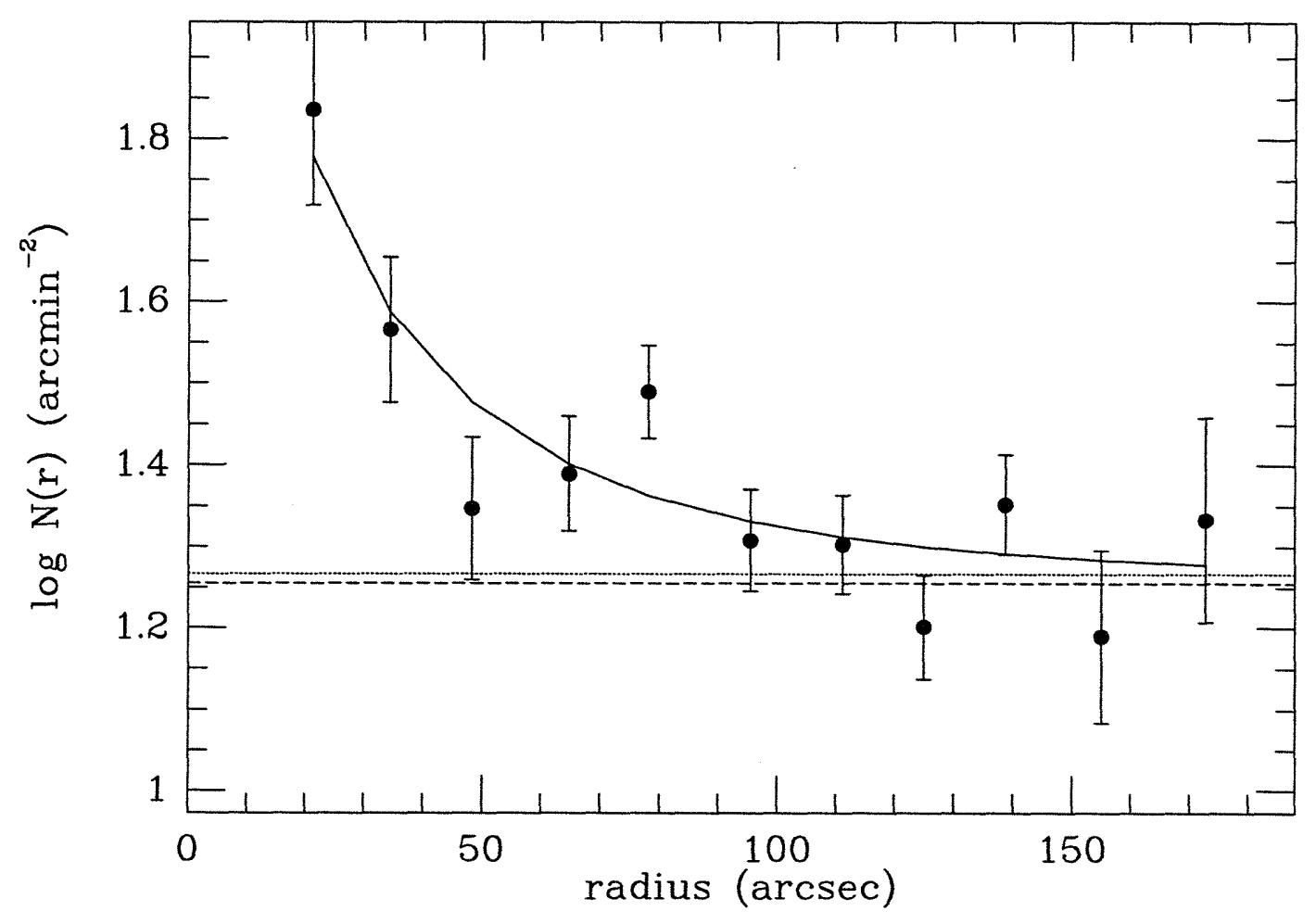

Figure 5-17: A2197-1. 


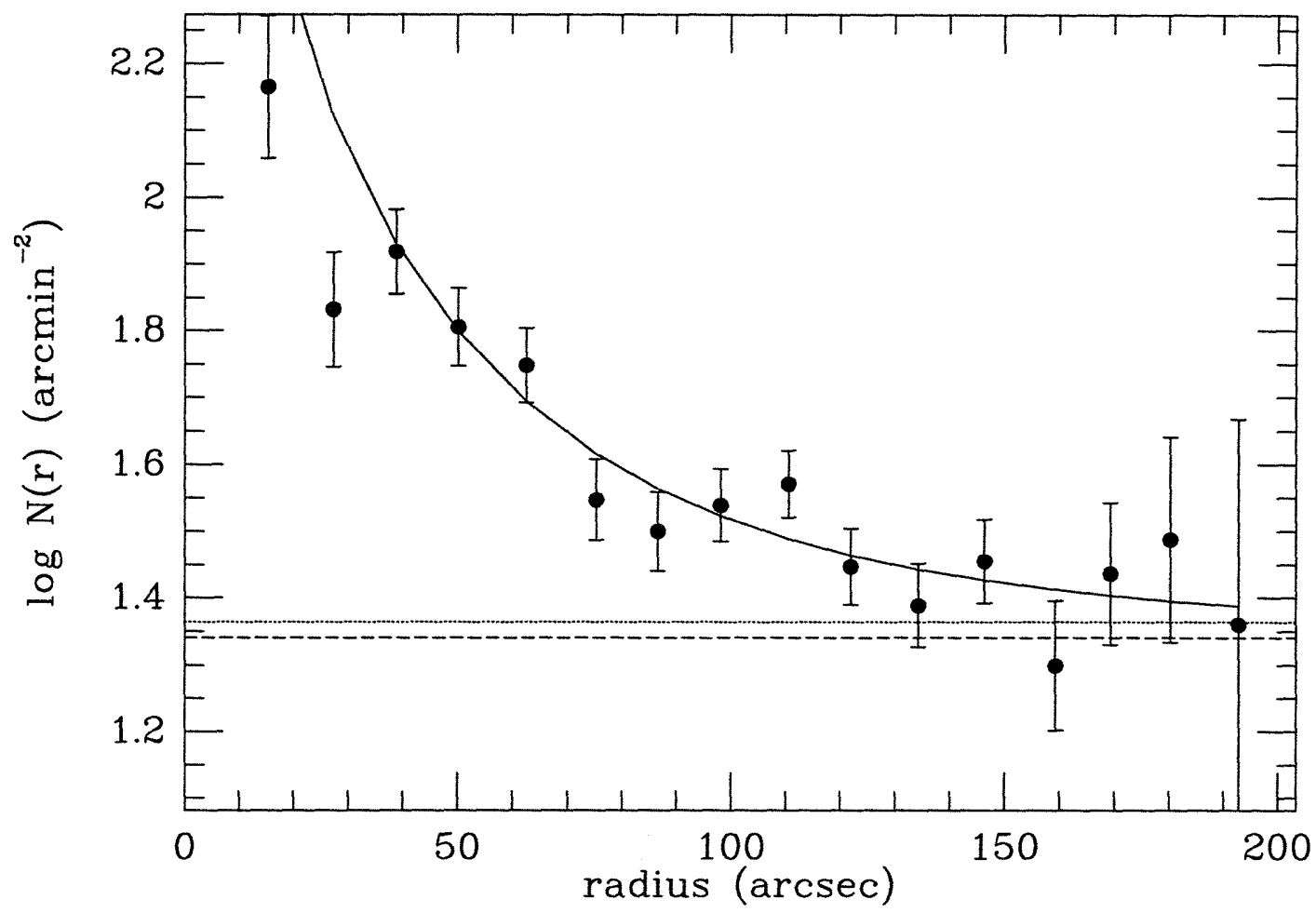

Figure 5-18: A2197-2.

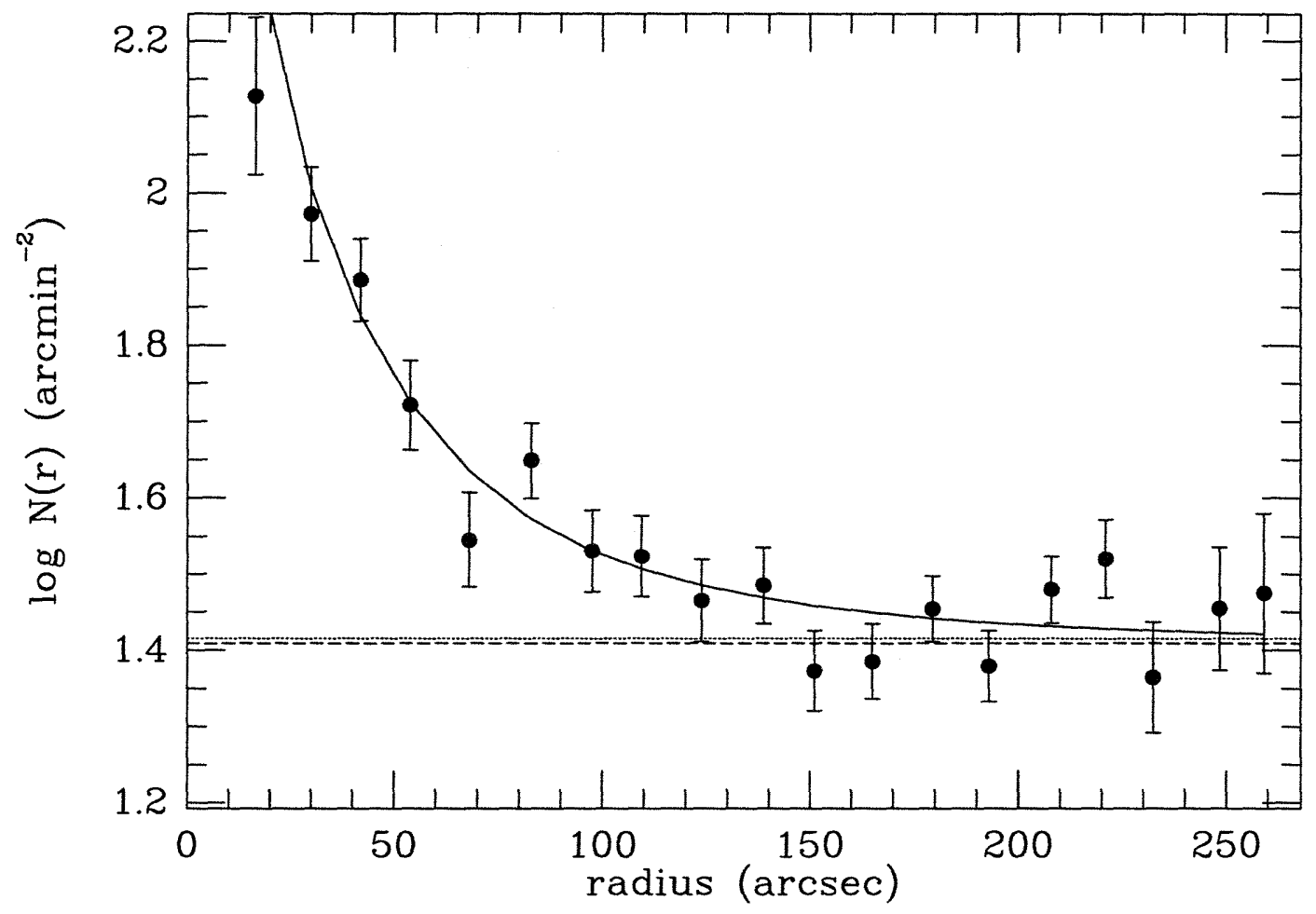

Figure 5-19: A2199-1. 


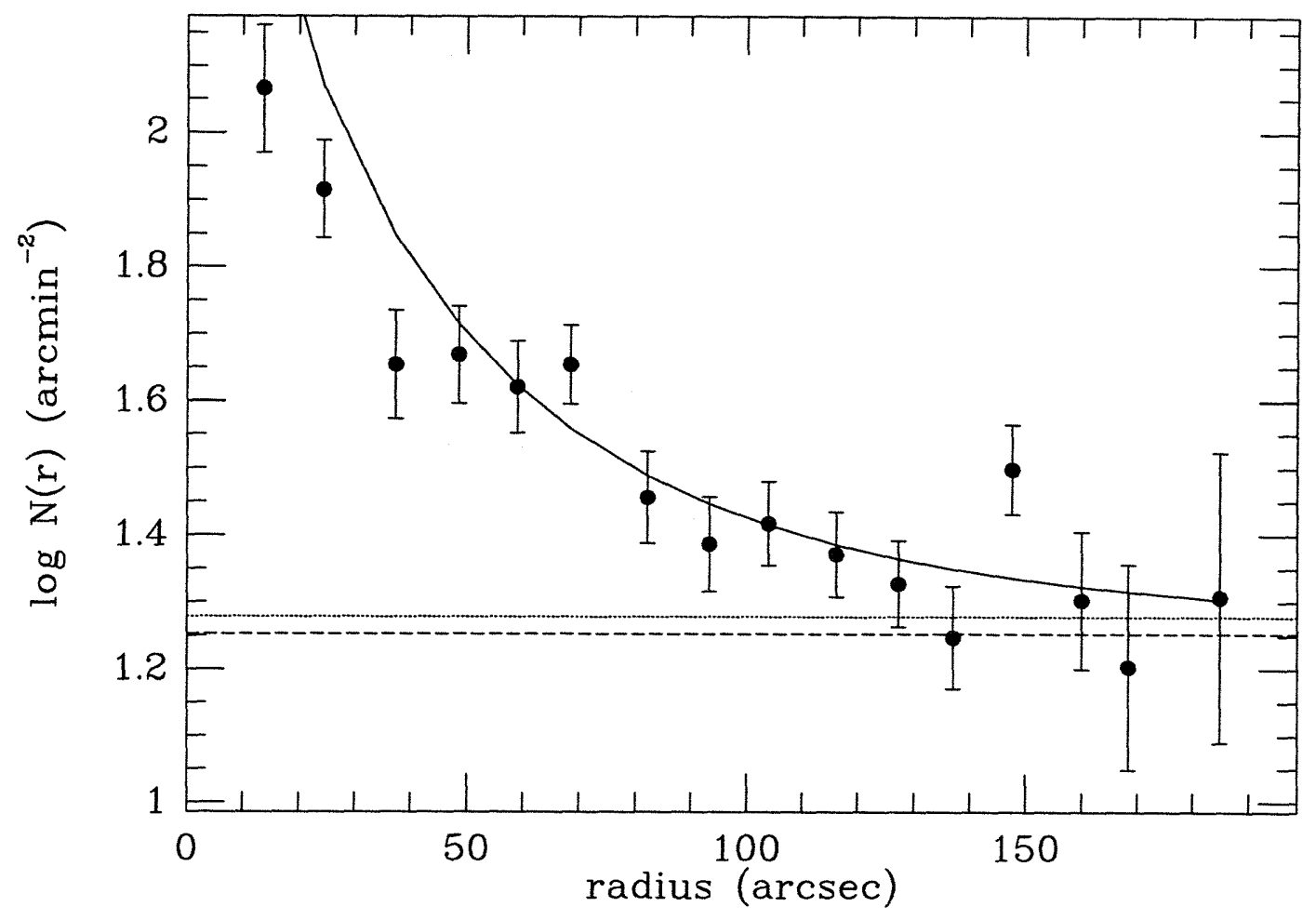

Figure 5-20: A2634-1.

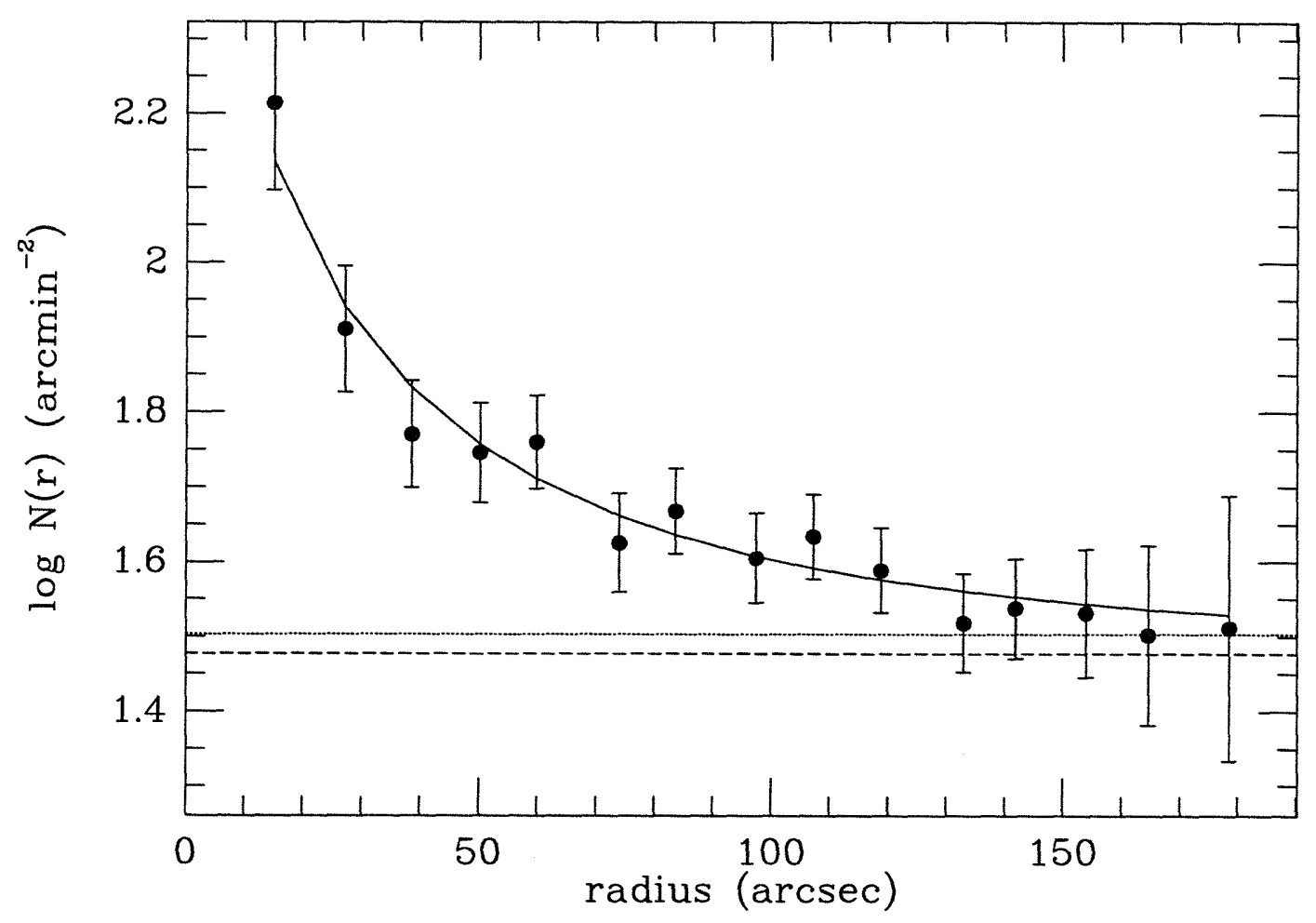

Figure 5-21: A2666-1. 


\subsubsection{Globular Cluster and Background Variances}

The mathematics for this section was developed in $§ 2.3 .1$; here, we describe the corrections for background variance applied in this sample. Two sources of contamination must taken into account: the stellar SBF and the fluctuations due to background galaxies. These must be subtracted from the $P_{0}$ measurements described in $\S 4.4 .3$ in order to determine the amplitude of the fluctuations due to GCs.

Most of the observations reported here are in the $R$ band, unlike the Coma $I$-band observations of Chapter 2, but the correction is done in the same way, relative to the measured value of the fluctuation magnitude in Virgo, where $\bar{m}_{R}=31.25$ for the BCG NGC 4472 and other big ellipticals, with a scatter of $\lesssim 0.20$ mag (Tonry et al. 1990), which is added in quadrature to a $\sim 0.25$ mag uncertainty ( $\$ 5.1 .3)$ in the relative distances of the program galaxies with respect to Virgo.

While the mean $(R-I)$ color of the GCs is about 0.55 (Ajhar, Blakeslee, \& Tonry 1994), the color of the SBF is $\left(\bar{m}_{R}-\bar{m}_{I}\right) \approx 1.6$. Thus, for a fixed value of $\left(m_{c}-m^{0}\right)$, the ratio calculated in equation 2.16 improves a factor of

$$
\begin{aligned}
\left(\frac{\sigma_{G C S}^{2}}{\sigma_{S B F}^{2}}\right)_{R} \times\left(\frac{\sigma_{G C S}^{2}}{\sigma_{S B F}^{2}}\right)_{I}^{-1} & \approx 10^{-0.4\left[2(V-I)_{G C}-\left(\bar{m}_{R}-\bar{m}_{I}\right)\right]} \\
& \approx 1.6 .
\end{aligned}
$$

The GC fluctuations in the $R$ band are $60 \%$ stronger relative to the SBF than they are in the $I$ band. (It is purely coincidental that the improvement factor precisely equals the SBF color.) Otherwise, this ratio of these fluctuation powers scales with $m_{c}$ and distance modulus as shown in Figure 2-1.

As in Tonry et al. (1997), we applied $K$-corrections to the fluctuation magnitudes to account for the slightly different region of the rest frame spectrum that passes through the imaging filter as a function of galaxy redshift. The corrections were based on calculations done by Worthey (1996), who kindly redshifted his published stellar populations models (Worthey 1993, 1994). Unlike for the SBF Survey (Tonry et al. 1997), where $K_{I}(z)=7 \times z$ was used for the $\bar{m}_{I} K$-correction, the correction here was not well described by a linear function out to the redshift limit of this sample; 
however, a quadratic form proved adequate. The effect of the $K$-correction in the $R$ band is to make the estimate of the contamination from the stellar SBF smaller smaller by $8 \%$ at $5000 \mathrm{~km} \mathrm{~s}^{-1}$ and by $13 \%$ at $10,000 \mathrm{~km} \mathrm{~s}^{-1}$, the limit of this survey. In practice, the stellar SBF contamination is only $\sim 10 \%$, so this small correction to it does not change the final results in any significant way, but it is important for avoiding bias. The uncertainty in the $K$-correction based on the scatter in Worthey's models was included in the calculation of our errors.

The larger correction $\left(\sim 10-50 \%\right.$, depending on $\left(m_{c}-m^{0}\right)$ and radius in the galaxy) to the measured variance is the one applied to account for the faint background galaxies. As in the SBF Survey (Tonry et al. 1990, 1997) and Chapter 2, this is done by extrapolating the results from maximum likelihood routine (to be described in full detail by Ajhar et al. [1997]) which fits the magnitude distribution of the galaxies to a power law in the outer parts of the image, taking into account the fact that the GCs may also extend out that far. This background estimation can be a difficult and uncertain procedure when the galaxy nearly fills the image, but in such cases the relative size of correction will be smaller. Out of necessity, the slope of the galaxy magnitude distribution is taken from Tyson (1988), and the normalization $T_{n}$ is fitted relative to his counts over the faintest few magnitudes of the image, in order to avoid the dwarf satellites of the central galaxy. Figure 5-22 shows two example outputs from the maximum likelihood program, and Table 5.2 lists the fitted values of $T_{n}$ and their uncertainties. From these measurements, the variance due to background galaxies is calculated according to equation 2.9 and subtracted along with the SBF estimate from the measured variance $P_{0}$ in order to obtain $P_{G C}$, the variance due to GCs. Our measurements of $P_{0}$ and $P_{G C}$ were listed in Table 5.1.

\subsubsection{Estimating $m^{0}$}

In Chapter 3 we raised some doubts concerning the universality of the GCLF turnover absolute magnitude $M^{0}$, but here we are going to have to estimate its value to some level of precision in order to derive the total number of GCs around each galaxy, and thence, the specific frequency $S_{N}$. Recall that Chapter 3 ended on the hopeful note 

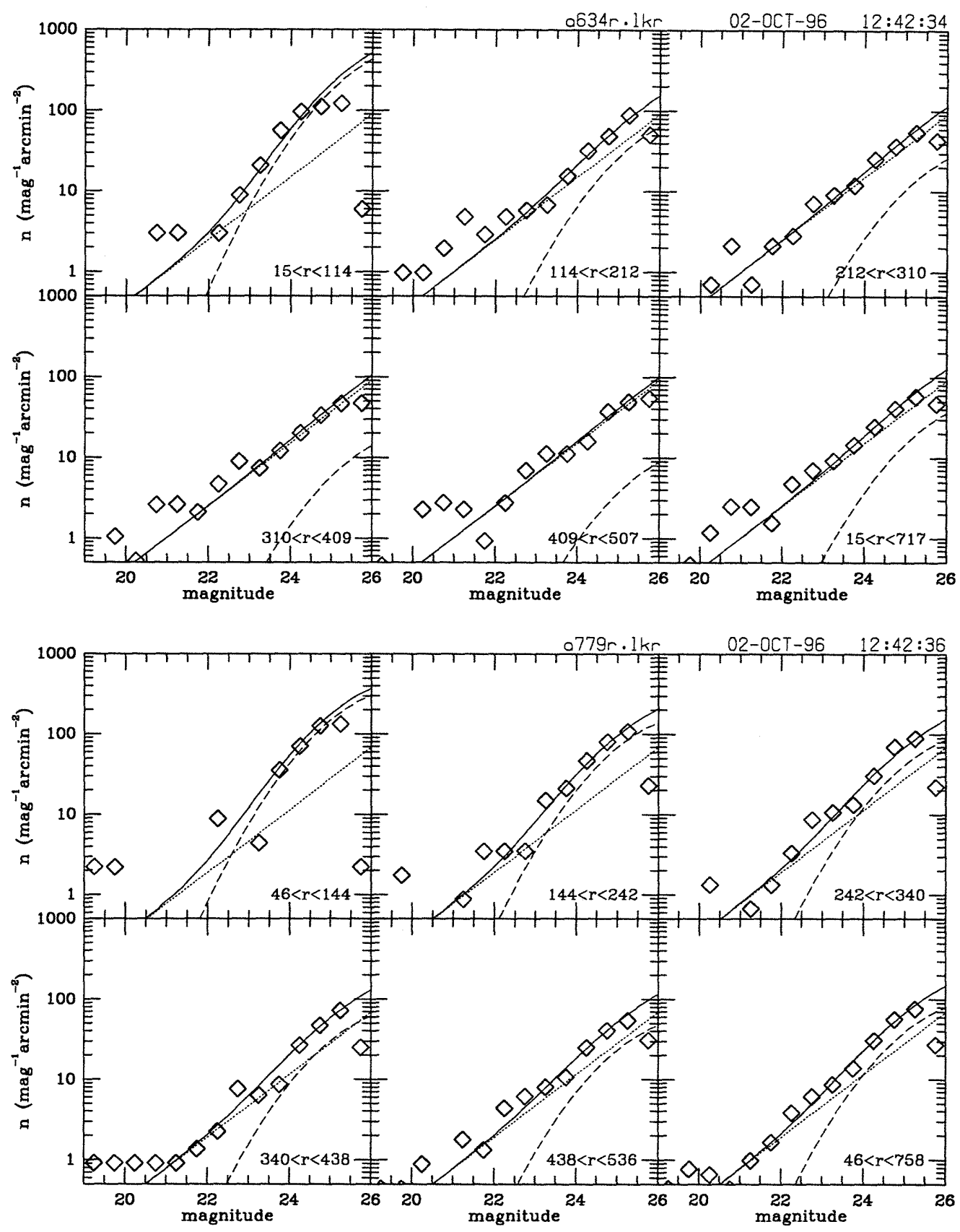

Figure 5-22: Two example outputs from the maximum likelihood background galaxy estimator are shown. The diamonds represent counts of all objects, extended and point sources. Dashed curves represent the M87 GCLF translated to the distances of these galaxies according to their CMB velocities, but with normalization fitted as a free parameter. Dotted lines represent the background galaxy distribution of fixed slope, but free normalization $T_{n}$. Solid curves are the sum of the two. The pixel range of each panel $\left(0.275 \mathrm{pix}^{-1}\right)$ is indicated, with last panels being sums. The top panel shows an "easy" case, A634-1, where few GCs remain at the image edge, while the lower panel shows a "hard" case, A779-1, where a significant number of GCs are found all the way out (although the galaxies are found to have similar values of $S_{N}$ ). 
Table 5.2: Background Galaxy Count Normalizations

\begin{tabular}{ccc|ccc|ccc}
\hline \hline Galaxy & $T_{n}$ & \pm & Galaxy & $T_{n}$ & \pm & Galaxy & $T_{n}$ & \pm \\
\hline A262-1 & 1.40 & 0.65 & A $779-1$ & 1.12 & 0.25 & A1656-3 & 1.15 & 0.40 \\
A347-1 & 1.50 & 0.40 & A $999-1$ & 1.44 & 0.35 & A2162-1 & 1.38 & 0.30 \\
A $397-1$ & 0.83 & 0.18 & A1016-1 & 1.33 & 0.30 & $\mathrm{~A} 2197-1$ & 1.50 & 0.55 \\
A539-1 & 1.50 & 0.55 & $\mathrm{~A} 1177-1$ & 1.33 & 0.25 & $\mathrm{~A} 2197-2$ & 0.95 & 0.25 \\
A539-2 & 1.75 & 0.65 & $\mathrm{~A} 1185-1$ & 1.00 & 0.20 & $\mathrm{~A} 2199-1$ & 1.00 & 0.22 \\
$\mathrm{~A} 569-1$ & 1.40 & 0.50 & $\mathrm{~A} 1314-1$ & 1.33 & 0.25 & $\mathrm{~A} 2634-1$ & 1.20 & 0.35 \\
$\mathrm{~A} 634-1$ & 1.50 & 0.30 & $\mathrm{~A} 1367-1$ & 1.00 & 0.35 & $\mathrm{~A} 2666-1$ & 1.15 & 0.40 \\
\hline \hline
\end{tabular}

that, if studied consistently within similar environments, the observed $m^{0}$ should go as the relative distance modulus. We shall proceed for now with that hope, then examine the results of this analysis to see if it is justified.

The value of $m_{V}^{0}$ in Virgo is well known from HST and ground-based observations, $m_{V}^{0}$ (Virgo $)=23.75 \pm 0.05$. Transforming to the $R$ band increases the uncertainty to $\sim 0.07$ mag. Although Virgo is too near to have been included in the Abell Catalog, its galaxy density is equivalent to that of a richness class 1 Abell cluster (Girardi et al. 1995); therefore, it is valid to use as a calibrating object. (Fornax, on the hand, is not rich enough; it shows up as S373 in the supplementary catalog of southern poor clusters tabulated by Abell, Corwin, \& Olowin [1989].) The CMB velocity of Virgo is derived from the Coma $\mathrm{CMB}$ velocity and the relative Coma-Virgo distance to be $v_{C M B}=1310 \pm 75 \mathrm{~km} \mathrm{~s}^{-1}$, contributing an uncertainty of $0.12 \mathrm{mag}$ to the calibration. Combining the two sources of uncertainty in quadrature yields an estimated error in the $m^{0}$ calibration of $\sim 0.15$ mag. This systematic uncertainty will be left out of the error calculations for now, but we will discuss its effects later.

As discussed in Chapter $3, M^{0}$ appears constant to within 0.1 mag in Fornax and to within $0.2 \mathrm{mag}$ or less in Virgo. We adopt a value of $0.2 \mathrm{mag}$ as a best estimate of the intrinsic dispersion in $M^{0}$ among giant ellipticals in clusters. It is also necessary to consider the possibility of Abell cluster radial peculiar velocities with respect to the CMB frame; these will have an effect on both $m^{0}$ and the estimated galaxy luminosity. There is a substantial literature on this subject, with one of the first modern works being that of Aaronson et al. (1986). Combining those results with subsequent 
observations, Aaronson et al. (1989) concluded that the rms of the one dimensional peculiar velocities of 20 clusters with Tully-Fisher distances was $<400 \mathrm{~km} \mathrm{~s}^{-1}$, with a few nearby outliers probably affected by the Great Attractor. Studies of the three dimensional correlation function of Abell and southern rich clusters (Huchra et al. 1990; Postman et al. 1992; Nichol et al. 1992) placed firm upper limits on the rms peculiar velocities of single clusters which ranged from 600 to $1000 \mathrm{~km} \mathrm{~s}^{-1}$, finding no measurable lower limits. Using $D_{n^{-}} \sigma$ measurements and reviewing the literature, Lucey et al. (1991) concluded that very few clusters had peculiar velocity measurements which differed significantly from zero, and those that did were probably as a result of $D_{n}-\sigma$ zero-point problems.

More recent work has provided measurements of the cluster peculiar velocities instead of upper limits. Zucca et al. (1993) used the arithmetic mean of the magnitudes of the third and tenth brightest galaxies in $1607 \mathrm{Abell} / \mathrm{ACO}$ clusters with measured redshifts to estimate their distances, after a correction for cluster richness, and gave a "best estimate" of $v_{r m s}^{\text {pec }}=500 \mathrm{~km} \mathrm{~s}^{-1}$, ruling out $1000 \mathrm{~km} \mathrm{~s}^{-1}$ at the $2 \sigma$ level but finding that zero rms peculiar velocity was allowable. Bahcall \& Oh (1996) have studied the whole "peculiar velocity function" of a sample of clusters with peculiar velocities derived from Tully-Fisher distances to member Sc galaxies. They determined the rms cluster peculiar velocity to be $293 \pm 28 \mathrm{~km} \mathrm{~s}^{-1}$, with no large tail of outliers, and a probability of $\lesssim 5 \%$ of finding a cluster whose radial velocity deviates from the Hubble flow by more than $600 \mathrm{~km} \mathrm{~s}^{-1}$. Bahcall \& $\mathrm{Oh}$ note that their result is significantly smaller than the expected value of $\sim 500 \mathrm{~km} \mathrm{~s}^{-1}$ for $\Omega=1$ CDM cosmogonies.

In this work, a compromise value of $400 \mathrm{~km} \mathrm{~s}^{-1}$ will be adopted for the rms one dimensional peculiar velocities of Abell clusters in the CMB frame. Thus, the final random uncertainty in the estimated $m^{0}$ values for our sample galaxies is

$$
\begin{aligned}
\delta m^{0} & = \pm \sqrt{0.20^{2}+\left(\frac{5}{\ln 10} \times \frac{400}{c z_{C M B}}\right)^{2}} \\
& \approx \pm 0.26 \mathrm{mag}, \text { at } 5,000 \mathrm{~km} \mathrm{~s}^{-1} \\
& \approx \pm 0.22 \mathrm{mag}, \text { at } 10,000 \mathrm{~km} \mathrm{~s}^{-1}
\end{aligned}
$$


The $\chi^{2}$ minimization described below is done first using the estimate of $m^{0}$ in each cluster according to its $\mathrm{CMB}$ velocity and equation 4.7 ; then $m^{0}$ is varied according to equation 5.3, and the error resulting from the (random) $m^{0}$ uncertainty is added in quadrature to the internal error from the $\chi^{2}$ minimization. Standard $K$-corrections (Schneider et al. 1983a) were applied to the estimates of $m^{0}$ before calculating the expected counts and variance from each model distribution, but these are very small, amounting to just $0.03 \mathrm{mag}$ in $R$ and $0.025 \mathrm{mag}$ in $I$ at the limit of the survey; the same corrections are made to the galaxy light, as it is has roughly the same color.

\subsubsection{Constraining $S_{N}$ and $\sigma$}

We follow the identical $\chi^{2}$ minimization procedure as in Chapter 2. For each of the four radial regions of each galaxy, we use the corrected variances and counts, shown in Table 5.1, to calculate the $\chi^{2}$ values for a grid of points in the $N_{0^{-}} \sigma$ plane. Here, as elsewhere, $N_{0}$ is the magnitude-integrated surface density of GCs and $\sigma$ is the Gaussian width of the GCLF; thus, the grid points represent model GCLFs of the same $m^{0}$, set according to the dictates of the previous subsection, but differing normalizations and widths. For displaying the results, we divide $N_{0}$ by the luminosity of the region in the proper units to yield the local specific frequency $S_{N}$ (the distance zero point is discussed in \$5.2.2). Appendix A displays the resulting probability contours for each region of each galaxy, analogous to Figures 2-5 and 2-6 for the $I$-band Coma observations. The next section presents the results of this analysis.

\subsection{Results}

\subsubsection{GCLF Widths}

To determine final $\sigma$ values for each galaxy, we average the results of the regions which provide useful constraints on $\sigma$, then vary $m^{0}$ within the limits of uncertainty, re-minimize $\chi^{2}$ for each region, and re-average. The resulting variation in $\sigma$, usually comparable to the internal error, is then included in the final error estimate.

Figure 5-23 plots the derived $\sigma$ values from this analysis against their uncertainties. 
The Coma galaxies from Chapter 2 are included in the figure. (For values with asymmetric errorbars, the average of the errors is used.) The results cluster near $\sigma=1.4 \mathrm{mag}$ when the uncertainty is small, but drift higher when the uncertainty becomes large. Table 5.3 in the following section lists the individual values. The overall weighted mean is $\langle\sigma\rangle=1.45 \pm 0.03 \mathrm{mag}$, the median is $1.46 \mathrm{mag}$, and the unweighted mean is $1.49 \mathrm{mag}$ with a dispersion of $0.13 \mathrm{mag}$. (Without the Chapter 2 galaxies, the weighted mean, median, and unweighted values become 1.46, 1.49, and $1.50 \mathrm{mag}$, respectively, with the same dispersion.)

However, if we exclude the values of $\sigma$ with uncertainties greater than $0.15 \mathrm{mag}$, where the upward bias appears to set in, the weighted mean is $\langle\sigma\rangle=1.43 \pm 0.03 \mathrm{mag}$, and the median and unweighted mean are both $1.42 \mathrm{mag}$, with a 0.07 mag dispersion. (Excluding the Chapter 2 galaxies now makes no difference in these numbers.) We tentatively conclude from the well-constrained measurements that the mean Gaussian GCLF width for the sample galaxies is around $1.43 \mathrm{mag}$, with a scatter consistent with the measurement errors, i.e., the GCLF width appears to be universal. However, the more poorly determined values are biased towards larger $\sigma$, which causes one to wonder if there might also be some bias remaining in the mean of low-error results. We consult the literature for insight into this question, then offer an explanation as to the most likely cause of the bias.

The primary GCLF calibrator for this analysis was M87, which is known to have $\sigma=1.40 \pm 0.06 \mathrm{mag}$ from HST measurements (Whitmore et al. 1995). Other wellmeasured GCLFs which provide reasonable comparisons for our sample are those of the Virgo BCG NGC 4472, $\sigma=1.47 \pm 0.10$ mag (Secker \& Harris 1993); the Fornax cD NGC 1399, $\sigma=1.38 \pm 0.09$ (Chapter 3); the giant Virgo background elliptical NGC 4365, $\sigma=1.41 \pm 0.15$ (Forbes 1996, HST measurement); and NGC 5846, the central giant elliptical in a compact group, $\sigma=1.34 \pm 0.06$ (Forbes et al. 1996b, also with HST). Thus, we expect the galaxies in this sample to have $\sigma$ near $1.40 \mathrm{mag}$, as we found in the initial application of this method in Chapter 2, and as found above for the well-constrained measurements. (We also found $\sigma=1.70 \mathrm{mag}$ for M87 in Chapter 2, but that was because we fixed its $m^{0}$ to be $0.45 \mathrm{mag}$ too faint, based 


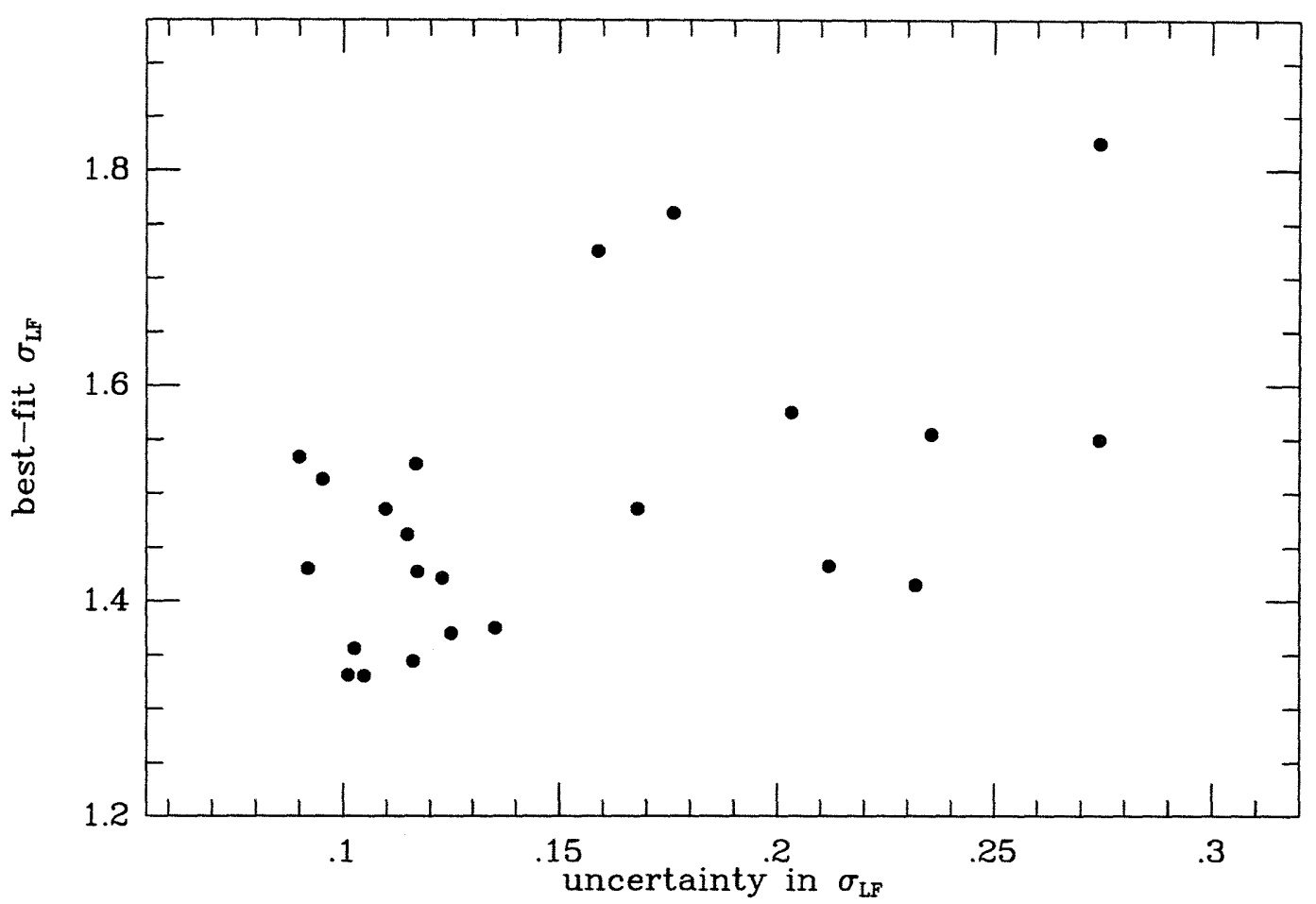

Figure 5-23: The derived GCLF width $\sigma$ in the CMB frame is plotted against its uncertainty, showing that the more uncertain values tend to biased high. See text for details.

on the McLaughlin et al. [1994] results; setting $m^{0}$ to the proper value yielded a $\sigma$ of $1.45 \mathrm{mag})$. It is true that published results from heroic efforts with barely adequate data tend to find GCLF widths that are too large (e.g., McLaughlin et al. 1994; Harris, et al. 1991), as compared with later studies (e.g., Whitmore et al. 1995; Forbes 1996); however, those measurements were based on Gaussian fits to the bright half of the GCLFs, so the relationship to the present bias is not clear.

In recent years, other functional forms besides Gaussians have been explored for use in fitting the GCLF. The most commonly used alternative is the " $t_{5}$ " distribution proposed by Secker (1992), but intersecting exponentials (Baum et al. 1995) and Gauss-Hermite expansions with two correction terms (Abraham \& van den Bergh 1995) have also been tried. What these alternate forms have in common is that they all have broader tails than a Gaussian, i.e., they are all "wingy", and they were all motivated by the smaller GCSs (200-300 members) of the Milky Way and M31. The GCLFs of large ellipticals with many thousand GCs, such as M87 (Whitmore et al. 
1995) and NGC 1399 (Chapter 3), are often exquisite Gaussians without enhanced tails. Thus, we would expect the GCLFs of our program galaxies to be Gaussian. As Abraham \& van den Bergh conclude, "for most applications, a simple Gaussian description is an adequate representation of the data."

However, the galaxies in our sample with poorly constrained and obviously biased measured values of $\sigma$ are the ones which are either among the smallest and relatively poorest in GCs (A634-1, A1016-1) or, in the case of A2162-1, suffered from poor seeing so that only the very brightest members far out in the tail contributed to the direct counts. If the counts are enhanced relative to the Gaussian model used for the $\chi^{2}$ minimization, the derived $\sigma$ will be too large. This was pointed out in $\S 2.3 .4$ and graphically illustrated in Figure 2-4. We believe this to be the most likely explanation for the bias in the under-constrained widths. A direct comparison in the following section of $S_{N}$ derived from counts and fluctuations with fixed $\sigma$ supports this view. The galaxies for which the counts penetrated to greater depths along the GCLF, or have no excess of bright GCs relative to a Gaussian, will not be similarly biased. The fact that the median $\sigma$ of the low-error measurements is $1.42 \mathrm{mag}$, virtually identical to the M87 calibrating GCLF value of 1.40 mag, supports this view. Moreover, it indicates that the working hypothesis of a universal GCLF for central galaxies in rich clusters has been vindicated (although the apparent difference in $M^{0}$ between clusters and poor groups remains).

To summarize this section, we have measured the GCLF widths of the Abell cluster galaxies in this sample. Although several of the under-constrained values are biased high, the remainder appear to be accurate. We find no evidence for any intrinsic dispersion among these better measured GCLF widths, and their average is very close to the value for $\mathrm{M} 87$, whose turnover magnitude $m^{0}$ and CMB velocity were used to estimate $m^{0}$ for the program galaxies, thereby allowing us to derive the GCLF widths. This result provides a valuable consistency check in support of the assumption of a universal GCLF for bright ellipticals in Abell clusters.

In the previous section, we estimated the systematic uncertainty in the $m^{0}$ calibration to be $\pm 0.15 \mathrm{mag}$, mainly due to the uncertainty in the CMB velocity of 
Virgo. Varying the estimates of $m^{0}$ by this amount has the effect of changing the individual GCLF widths by 0.04-0.06 mag. Thus, we conclude that the mean of the GCLF widths with internal uncertainties smaller than 0.15 mag in this sample is $\langle\sigma\rangle=1.43 \pm 0.03 \pm 0.05 \mathrm{mag}$, where the first errorbar reflect the internal error and the second indicates the systematic uncertainty.

Because we find no evidence of significant differences in GCLF $\sigma$ between M87 and the sample galaxies or among the sample galaxies themselves, we report specific frequencies in the following section assuming the HST M87 value of $\sigma=1.40$ mag. For an intrinsic " $1 \sigma$ " dispersion in this value, we assume $\pm 0.05 \mathrm{mag}$, consistent with our low-error results (which are in fact consistent with no intrinsic dispersion) and with the scatter in the measured widths of nearby dominant ellipticals. This approach was chosen because the derived $S_{N}$ is strongly correlated, in a negative sense, with the value of $\sigma$, as evidenced by the figures shown in the appendix. In this way, we hope to avoid biased intercomparisons among the $S_{N}$ results for these galaxies.

\subsubsection{Specific Frequencies}

In order to calculate $S_{N}$, it is necessary to adopt a zero point for the distance scale. Until now, all magnitudes have been set relative to their observed values in Virgo, using the Coma-Virgo relative distance to place Virgo in the CMB frame. This would appear to be the moment of truth in which we ally ourselves with either the "long" or "short" distance scale advocates. In fact, it is of no consequence, since any observed trends of $S_{N}$ will be independent of the zero point. However, something must be used, and we see no reason to turn a blind eye on all the recent Cepheid observations in the neighborhood of Virgo. Thus, we adopt a Virgo distance modulus of 31.02 (negligibly different from the 31.0 of previous chapters) because there are four Virgo spirals with Cepheid distance moduli clustered around this average value (van den Bergh 1996), although there is also one which is 1 mag more distant. To transform the $S_{N}$ values presented here to another distance scale, simply multiply them all by $10^{0.4\left[31.02-(m-M)_{0}\right]}$, where $(m-M)_{0}$ is one's own favorite Virgo distance modulus. (The $S_{N}$ values will be systematically smaller for a larger Virgo distance.) 
Table 5.3: Metric Specific Frequencies and GCLF Widths in CMB Frame

\begin{tabular}{|c|c|c|c|c|c|}
\hline Galaxy & $S_{N} \pm$ & $\sigma_{\mathrm{LF}} \pm$ & Galaxy & $S_{N} \pm$ & $\sigma_{\mathrm{LF}} \pm$ \\
\hline A262-1 & $5.0_{1.3}^{1.5}$ & $1.38: .14$ & A1314-1 & $4.2_{1.0}^{1.1}$ & 1.58 .21 \\
\hline A $347-1$ & $5.8_{1.3}^{1.6}$ & $1.36: .10$ & A1367-1 & $5.3_{1.1}^{1.4}$ & 1.53 .11 \\
\hline A $397-1$ & $4.7_{1.1}^{1.4}$ & $1.43 . .11$ & A1656-1 & $5.7_{1.3}^{1.3}$ & $1.37: .12$ \\
\hline A539-1 & $9.1_{2.6}^{3.0}$ & $1.42 . .13$ & A1656-2 & $9.3_{2.0}^{2.0}$ & 1.43 .09 \\
\hline A539-2 & $9.4_{2.4}^{3.0}$ & $1.46: .12$ & A1656-3 & $4.6_{1.3}^{1.5}$ & 1.33 .11 \\
\hline A569-1 & $3.0_{1.0}^{1.2}$ & 1.42 .24 & A2162-1 & $7.4_{1.8}^{2.2}$ & 1.73 .16 \\
\hline A634-1 & $4.0_{1.0}^{1.2}$ & 1.76 .18 & A2197-1 & $2.5_{1.3}^{1.4}$ & $1.55 . .24$ \\
\hline A779-1 & $4.1_{0.9}^{1.0}$ & 1.34 .12 & A2197-2 & $5.9_{1.2}^{1.5}$ & 1.49 .11 \\
\hline A999-1 & $3.9_{1.3}^{1.5}$ & $1.56: .23$ & A2199-1 & $8.1_{1.9}^{2.3}$ & (.09 1.53 .10 \\
\hline A1016-1 & $3.3_{1.1}^{1.2}$ & $1.83 . .24$ & A2634-1 & $7.5_{1.7}^{2.1}$ & 1.51 .09 \\
\hline A1177-1 & $4.2_{1.0}^{1.3}$ & 1.49 .18 & A2666-1 & $3.5_{1.0}^{1.1}$ & 1.43 .24 \\
\hline A1185-1 & $6.4_{1.4}^{1.8}$ & 1.33 .10 & & & \\
\hline
\end{tabular}

Columns list: galaxy name; $S_{N}$ calculated within $32 h^{-1} \mathrm{kpc}$, assuming $\sigma=1.40 \pm 0.05$ for the GCLF width; actual measured value of $\sigma$.

For each radial region of each galaxy, we assumed $\sigma=1.4 \mathrm{mag}$ and calculated the total GC surface density $N_{0}$ from the counts of GCs brighter than the cutoff magnitude $m_{c}$ and from the fluctuations resulting from those fainter than this cutoff. The two separate measurements were then weighted averaged, and all the GCs between some inner radial limit, discussed below, and an outer radius of $32 h^{-1} \mathrm{kpc}$ were summed; the result was then divided by the normalized luminosity of the galaxy within the same radial range to derive the "metric $S_{N}$ " within $32 h^{-1} \mathrm{kpc}$. This was repeated for $\sigma=1.35$ and $1.45 \mathrm{mag}$, and then for $\sigma=1.4$ but with $m^{0}$ varied according to equation 5.3. (For $S_{N}$, unlike $\sigma$, the distance uncertainty due to random cluster velocities largely cancels, since it similarly effects both $N_{0}$ and the galaxy luminosity.) The variation in $S_{N}$ due to the uncertainties in these GCLF parameters was then added in quadrature to the internal errors which had been propagated from the counts and fluctuations. Table 5.3 lists the metric $S_{N}$ results, with their final errors, for each galaxy. Note that it also lists the derived values of $\sigma$ discussed in the previous section, but these are not the ones used in calculating the tabulated values of $S_{N}$. 
Table 5.4: Comparison of $S_{N}^{f l u}$ and $S_{N}^{c n t}$ for $\sigma=1.40 \mathrm{mag}$

\begin{tabular}{cccrcc|cccccc}
\hline \hline Galaxy & $S_{N}^{\text {flu }}$ & \pm & \multicolumn{1}{c}{$S_{N}^{\text {cnt }}$} & \pm & $\sigma_{\text {dev }}$ & Galaxy & $S_{N}^{\text {flu }}$ & \pm & \multicolumn{1}{c}{$S_{N}^{\text {cnt }}$} & \pm & $\sigma_{\text {dev }}$ \\
\hline $\mathrm{A} 262-1$ & 5.6 & 1.6 & 4.7 & 1.1 & 0.50 & $\mathrm{~A} 1185-1$ & 6.7 & 0.7 & 5.4 & 1.3 & 0.96 \\
$\mathrm{~A} 347-1$ & 6.8 & 1.3 & 5.0 & 1.1 & 1.18 & $\mathrm{~A} 1314-1$ & 3.4 & 0.7 & 6.7 & 1.1 & 2.65 \\
$\mathrm{~A} 397-1$ & 5.3 & 0.6 & 3.6 & 0.9 & 1.75 & $\mathrm{~A} 1367-1$ & 5.6 & 1.0 & 6.0 & 0.6 & 0.35 \\
$\mathrm{~A} 539-1$ & 8.2 & 1.9 & 10.3 & 2.4 & 0.69 & $\mathrm{~A} 1656-3$ & 5.7 & 1.1 & 3.8 & 1.0 & 1.29 \\
$\mathrm{~A} 539-2$ & 8.7 & 1.5 & 11.0 & 1.7 & 1.00 & $\mathrm{~A} 2162-1$ & 6.3 & 1.1 & 15.5 & 2.8 & 3.17 \\
$\mathrm{~A} 569-1$ & 3.9 & 2.1 & 2.7 & 1.5 & 0.46 & $\mathrm{~A} 2197-1$ & 1.8 & 1.5 & 3.7 & 1.9 & 0.80 \\
$\mathrm{~A} 634-1$ & 2.7 & 1.2 & 6.6 & 1.3 & 2.28 & $\mathrm{~A} 2197-2$ & 5.5 & 0.7 & 7.1 & 1.1 & 1.22 \\
$\mathrm{~A} 779-1$ & 4.4 & 0.6 & 3.7 & 0.6 & 0.96 & $\mathrm{~A} 2199-1$ & 7.5 & 0.8 & 11.2 & 1.4 & 2.37 \\
$\mathrm{~A} 999-1$ & 3.5 & 1.7 & 4.8 & 1.7 & 0.57 & $\mathrm{~A} 2634-1$ & 7.1 & 0.8 & 9.4 & 1.5 & 1.43 \\
$\mathrm{~A} 1016-1$ & 2.6 & 1.7 & 5.0 & 1.3 & 1.14 & $\mathrm{~A} 2666-1$ & 3.7 & 1.0 & 3.0 & 0.9 & 0.53 \\
$\mathrm{~A} 1177-1$ & 3.9 & 0.8 & 4.7 & 1.1 & 0.64 & & & & & & \\
\hline \hline
\end{tabular}

For reference, we list in Table 5.4 the specific frequency values $S_{N}^{f l u}$ and $S_{N}^{\text {cnt }}$ derived separately from the variance measurements and the counts, respectively, with GCLF $\sigma$ fixed at 1.40 mag. The table shows the internal measurement errors on these values. As they include no allowance for uncertainty in the GCLF $m^{0}$ or $\sigma$, these errors are uncorrelated. The value of the width $\sigma$ which would bring $S_{N}^{f l u}$ and $S_{N}^{c n t}$ into precise agreement for a given galaxy is the one listed in Table 5.3 for that galaxy. Table 5.4 also lists the number of standard deviations $\sigma_{d e v}$ separating the two $S_{N}$ values. Note that the four galaxies with $\sigma_{d e v}>2$ all have $S_{N}^{c n t}>S_{N}^{f l u}$ and best-fit $\sigma \geq 1.53 \mathrm{mag}$ (Table 5.3), consistent with the discussion in the previous section about the bias in the measured GCLF widths being due to counts which are enhanced relative to a Gaussian model. However, we did not feel that this would justify throwing away the information provided by the counts.

Estimates of the global $S_{N}$ involve two very uncertain extrapolations of the GCS and galaxy profiles out to large radii. Not only are small uncertainties magnified by the extrapolations, but there is often no guarantee that the profiles do not change outside the imaged field. Thus, we chose not to make such extrapolations. The decision to report metric values of $S_{N}$ (i.e., values derived within the same physical radius around each galaxy) was made in order to avoid a bias in the reported $S_{N}$ 
with redshift. The metric radius of $32 h^{-1} \mathrm{kpc}$ was chosen because it corresponds to the limit of the image for the nearest of the sample galaxies (roughly 500 pix, or 2.3 , in the $1024^{2} R$-band images). Finally, the inner radius varied a bit and was set by practical constraints from the variable quality of the model subtraction at small radii. In effect, the inner limit was the smallest usable radius, typically smaller (in angle on the sky) for the more distant galaxies, but never less than $10^{\prime \prime}$, corresponding roughly to the expected GCS core radius in the nearest galaxies (Forbes et al. 1996a). For A1177-1 and A2197-1, the inner radius was 20", and for A2199-1 it was nearly as large, due to the extreme messiness of the central regions of these galaxies. In any case, it makes little difference, as such a small portion of the GCS is involved.

The $S_{N}$ values listed in the table for A1656-1 (NGC 4889) and A1656-2 (NGC 4874) differ from the global values quoted in Chapter 2 for two reasons. First, the RC2 photometry (de Vaucouleurs et al. 1976) used there was in error. It gave a total magnitude for NGC 4874 that was substantially too faint, as well as a slightly too faint total magnitude for NGC 4889. However, since that chapter has been published (Blakeslee \& Tonry 1995) the numbers have not been altered. Using the photometry from the RC3 (de Vaucouleurs et al. 1991) yields global values of $S_{N}=10.2 \pm 2.4$ for NGC 4874 and $S_{N}=6.8 \pm 1.8$ for NGC 4889, closer to those shown in Table 5.3. Second, as stated above, the table gives the metric $S_{N}$ values within a limited radial range, using our own photometry of the galaxy light within that range. As the GC systems of those two galaxies were found to be more extended than the halo light, it is expected that their metric $S_{N}$ would be smaller than their global $S_{N}$, and we see from the table that this is the case.

It should be emphasized that the "metric $S_{N}$ " we have introduced is of a very different nature than other quantities traditionally discussed in "metric" terms. For instance, the metric luminosity is a strong function of the chosen metric radius, since it refers to the total amount of light within that physical distance from the galaxy center. $S_{N}$, on the other hand, is the number of GCs per unit galaxy luminosity, so if the GCs follow the same radial distribution as the halo light (true to at least zeroth order), the "metric $S_{N}$ " will actually be independent of the chosen metric radius and 
will be the same as the global $S_{N}$. However, it is often, but not always, the case that the GCs follow a more extended distribution (e.g., Harris 1991), and consequently, the metric $S_{N}$ will asymptotically approach the global value (or perhaps abruptly reach it, in the case of tidal truncation). Henceforward, by $S_{N}$ we will mean these metric specific frequencies, unless otherwise stated.

We conclude with some comments on the measured values of $S_{N}$ for these galaxies and their uncertainties. First, there is no separation of galaxies into "normal" and "anomalous" $S_{N}$ classes. They appear to vary uniformly between $S_{N} \sim 3$ and $S_{N} \sim 9$. Second, in the three clusters with more than one galaxy in this sample, the second ranked galaxies (SRGs) all have higher $S_{N}$ than the BCGs, although in the case of A539 the difference is not significant. This is a selection effect. The SRGs were included in this sample because they are all giant ellipticals that appear to dominate their clusters at least as much as the actual BCGs selected by LP. A quick look at Table 4.1 shows that they all have larger $\alpha$ parameters than their respective BCGs, and comparison of their coordinates with those in Table 4.2 shows that they are all closer to their cluster X-ray centers. The importance of these facts will become clear in the following sections.

We have attempted to keep the $S_{N}$ errors listed in Table 5.3 both realistic and independent. However, it is possible that they are not completely independent. If, for example, the intrinsic dispersion in $M^{0}$ is only $\sim 0.1 \mathrm{mag}$ instead of $\sim 0.2 \mathrm{mag}$, then the final errors will all be similarly overestimated, and not independent. It seems unlikely, though, that the intrinsic dispersion in the GCLF width is less than the 0.05 mag we have adopted. The uncertainties from the GCLF parameters dominate, being 1-3 times as large as the uncertainties due to measurement error, depending on the size of the GC population and the depth of the data. The tabulated errors are offered as best estimates of the uncertainties in the individual $S_{N}$ values, but due to a preference to err on the side of caution, they may be correlated for different galaxies.

Finally, we discuss the effects on the $S_{N}$ results of the estimated \pm 0.15 mag systematic uncertainty in the $m^{0}$ calibration. Increasing the individual $m^{0}$ estimates by 0.15 mag has the effect of increasing the derived $S_{N}$ values by $19 \pm 3 \%$; decreasing 
$m^{0}$ systematically by the same amount decreases the derived $S_{N}$ values by $15 \pm 2 \%$. Thus, the "top" of the $S_{N}$ continuum for this sample may move upward from 9.3 to 11.1 , as the "bottom" move from 3 to 3.6 , or the top and bottom may decrease together to 7.9 and 2.5 , respectively. These estimates assume that the distance moduli are held fixed; varying them along with $m^{0}$ decreases the leeway to just a few percent, but the relative uncertainties in the Virgo distance modulus and CMB velocity are similar in size.

\subsubsection{Correlations}

The $S_{N}$ values derived in the previous subsection are plotted below against various properties of the host galaxy and surrounding cluster. To avoid confusion, we note that throughout this section, as elsewhere, the word "cluster", when it appears alone, means "galaxy cluster"; the abbreviation "GC" refers to "globular cluster."

\subsubsection{1 $S_{N}$ vs Galaxy Properties}

The top panel of Figure 5-24 plots the derived $S_{N}$ values against absolute $R$-band metric magnitude of the galaxy from LP. The lack of any significant correlation is understandable, as the LP metric magnitude is calculated within $10 h^{-1} \mathrm{kpc}$, while these $S_{N}$ values were derived for the total usable area of the galaxy within $32 h^{-1} \mathrm{kpc}$. Therefore, in the lower panel of the figure we plot $S_{N}$ against the absolute $V$ magnitude (since $S_{N}$ is defined relative to $\mathrm{V}$ magnitude) of the region of each galaxy in which the plotted $S_{N}$ was derived. Here, there does appear to be a weak correlation, but the impression is based almost entirely on the four faintest galaxies, which

all have $S_{N} \lesssim 4$. Overall, the significance of the correlation is 0.96 , based on the Spearman rank-order correlation coefficient, but excluding the four faintest galaxies causes the significance level to drop to 0.40 (i.e., no correlation). Galaxy luminosity is apparently not the driving force behind variations in $S_{N}$ among these galaxies.

The panels of Figure 5-24 include the errorbars from Table 5.3, primarily to demonstrate their size, but the rest of the figures will omit them for the sake of clarity (possible correlated errors, discussed above, would tend to obscure any trends). Later 
figures will show that the scatter in $S_{N}$ is fairly small, but here there are no strong correlations for the errorbars to detract from.

High values of $S_{N}$ have been associated with the phenomenon of extended $\mathrm{cD}$ halos (e.g., Harris 1991, McLaughlin et al. 1994). Figure 5-25 shows $S_{N}$ plotted against two measures of galaxy extent, the profile structure parameter $\alpha$ (defined in $\S 4.1$ ), as listed by LP for the CMB frame, and the effective radius $R_{e}$ of the best fitting $r^{1 / 4}$-law, taken from Graham et al. (1996), who tabulated this quantity for the BCGs in the CMB frame with $H_{0}=80 \mathrm{~km} \mathrm{~s}^{-1} \mathrm{Mpc}^{-1}$. Where available, the effective radii for the SRGs were taken from the RC3 (de Vaucouleurs et al. 1991) and brought into consistency with Graham et al. via the effective radius of the BCG, tabulated by both sources. Both panels exhibit a general upward trend. The formal significance levels of the correlations are 0.998 and 0.990 for the upper and lower panels, respectively.

Since $\alpha$ measures the slope of the galaxy profile at fairly small radius, $R_{e}$ is probably a better measure of overall halo extent, but apart from two outliers with $S_{N}>7$ and $\alpha \sim 0.5$, the top panel has less scatter. The biggest outlier in the $\alpha$ plot is A539-1, which is not the dominant galaxy in A539, judged by its position relative to the cluster X-ray/dynamical center, although it does have many GCs. The other "outlier" is A2162-1, a cD galaxy whose extended halo only sets in at larger radius; it begins to move into line with the more extended galaxies in the lower panel. Although both of these plots show considerable scatter, they do support the view that $S_{N}$ and galaxy extent are somehow associated.

It is always advisable to look for systematic effects in final results; therefore in Figure 5-26 we have plotted our derived $S_{N}$ values against cluster redshift. These redshifts were the basis for our $m^{0}$ estimates and the galaxy luminosity calculations, but as the figure shows, they do not correlate with $S_{N}$.

\subsubsection{2 $S_{N}$ vs Cluster Properties}

Now we consider possible correlations of $S_{N}$ with properties of the clusters in which the galaxies reside. There are many different parameters to explore, including density, dynamics, morphology, and X-ray properties. Any of these could have an effect on 

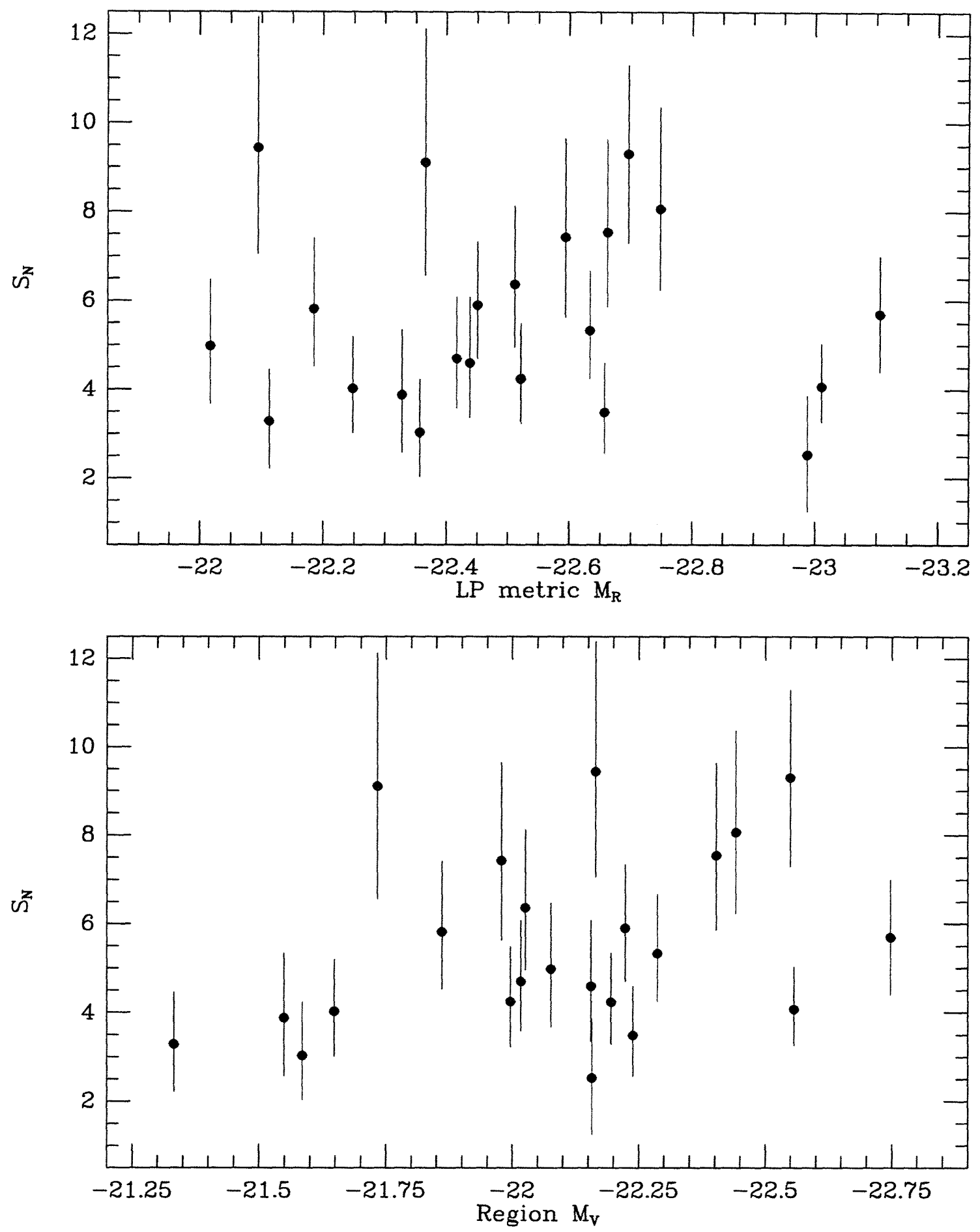

Figure 5-24: $S_{N}$ is plotted against $(a)$ metric absolute magnitude from Lauer \& Postman (1994), and (b) total absolute magnitude of the specific region of each galaxy in which $S_{N}$ was measured (i.e., from $\sim 3$ to $32 h^{-1} \mathrm{kpc}$ ). All quantities are calculated in the CMB frame. 

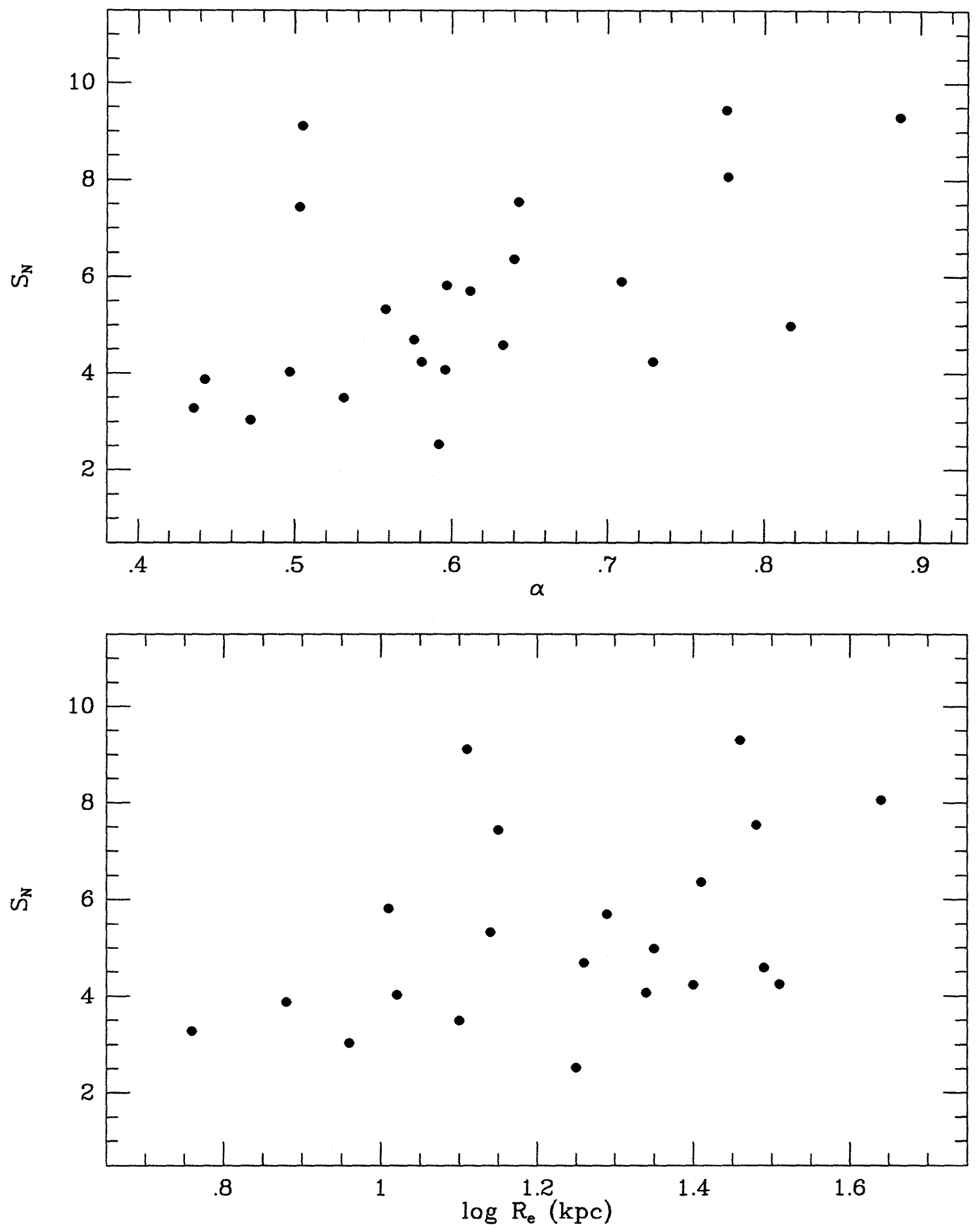

Figure 5-25: $S_{N}$ is plotted against two measures of the galaxy profile, $(a)$ the structure parameter $\alpha$ and (b) the logarithm of the effective radius $R_{e}$. Errorbars have been omitted for the sake of clarity. 


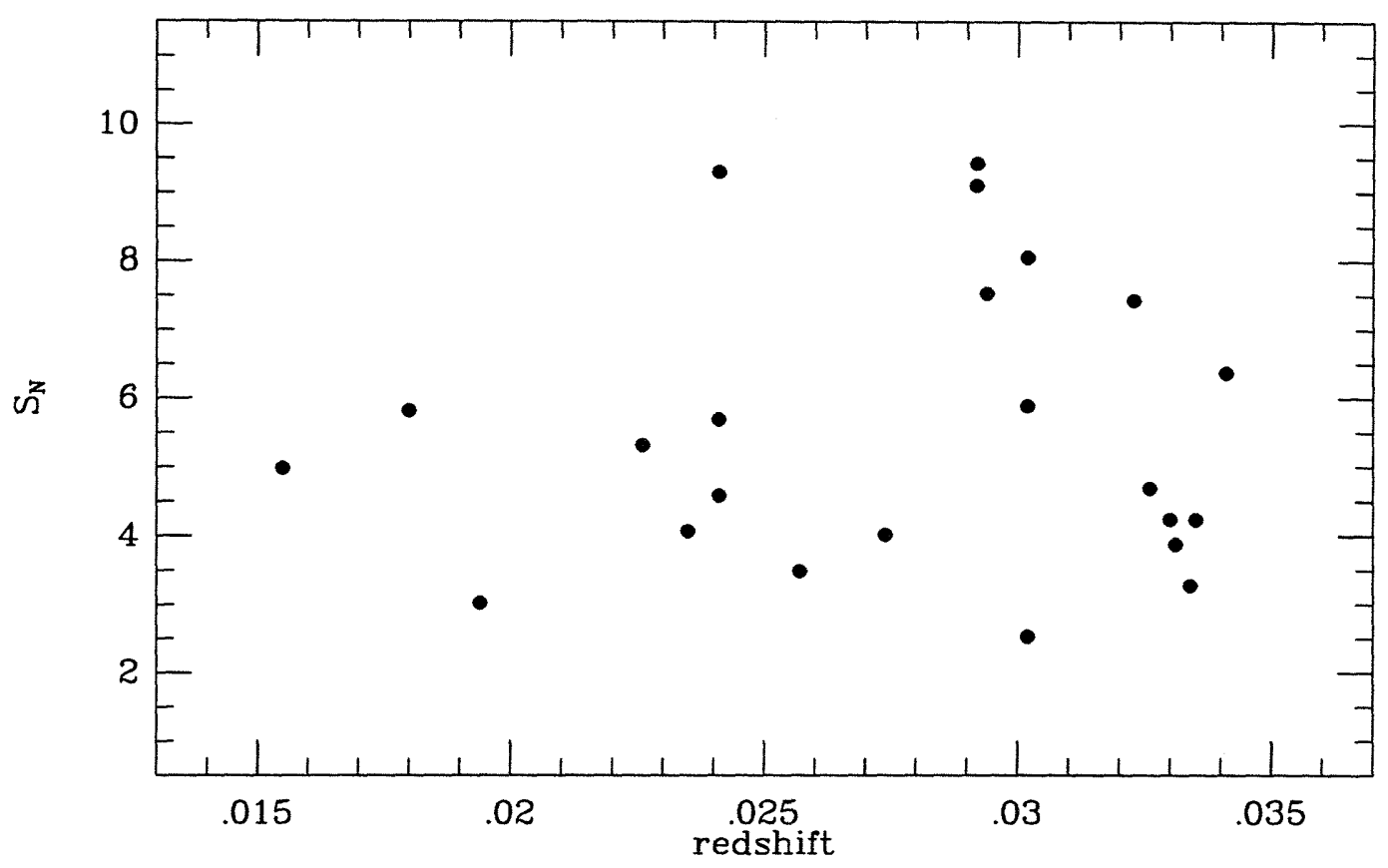

Figure 5-26: $S_{N}$ is plotted against the cluster redshift in the CMB frame. Though this is the redshift used in deriving the $S_{N}$ values, there is thankfully no correlation.

the GCS of the central galaxy in the cluster. We begin with the dynamics.

In collecting cluster velocity dispersions from the literature, one must take care to ensure that the final set is a fairly homogenous one. Girardi et al. (1993) found that the various methods of estimating dispersions give consistent results as long as the number of cluster members with measured redshifts exceeds about 20; less sophisticated, or "robust," analyses often yield erroneous results with fewer than 15 measured redshifts. This collection of dispersions, listed in Table 4.2, started with the "robust dispersions" determined by Girardi et al. (1993) from the data sets of Zabludoff et al. (1990) and Yahil \& Vidal (1977) for cluster galaxies within $1.5 h^{-1} \mathrm{Mpc}$ (the Abell radius, $r_{A}$ ) of the cluster centers. These dispersions have been superseded by the more recent measurements of Beers et al. (1991) for A569 and Zabludoff et al. (1993) for A1185, A1367, A1656, and A2199, both of whom likewise reported dispersions within $1 r_{A}$. To these have been added the dispersions for A2634 and A2666 from Scodeggio et al. (1995), who conducted a detailed study of these possibly interacting clusters. Because A2634 appears to be merging with a group of spirals at larger radius, and the smaller A2666 would otherwise be heavily 
contaminated by nearby A2634 galaxies, the values of $\sigma$ within half an Abell radius have been used, which should more accurately reflect the central potentials of these clusters. All of these dispersions are based on at least 22 cluster members with measured velocities (after outliers have been rejected); finally, Struble \& Rood (1991) list dispersions based on $>20$ member redshifts for three other clusters in the BCG sample, so these have been used as well. (Struble \& Rood also list a dispersion of $663 \mathrm{~km} \mathrm{~s}^{-1}$ for A1314 based on only 16 redshifts, but this is a factor of 2.4 larger than its "asymptotic dispersion" [described below]; no other cluster has a discrepancy as large as $50 \%$, indicating that this is an unreliable dispersion value.)

Figure 5-27 shows the resulting plot of $S_{N}$ against cluster velocity dispersion. There is a tight correlation, indicating that bright galaxies in regions of higher dispersion, and thus deeper potential wells, have more GCs per unit luminosity. The relation is reminiscent of the environment- $M_{V}^{0}$ correlation reported in Chapter 3 , but of an obviously greater significance. In this and most of the following figures, the more central galaxies (judged by the X-ray center) in the clusters with multiple members in this sample are the ones shown as filled symbols, while the less central ones are shown as open squares for de-emphasis, since they often deviate from the correlations. (The significance level here is 1.00 for the filled symbols.) The non-central galaxies will be referred to as "secondary", although three of the four are among the BCGs selected by LP, with the other one being NGC 4839, the third ranked galaxy in Coma.

Recently, Girardi and collaborators have been studying cluster velocity dispersion profiles (Fadda et al. 1996; Girardi et al. 1996), reporting the asymptotic values at large radii. They argue that "asymptotic dispersions" are less affected by small-scale velocity anisotropies, and thus more indicative of the depth of the cluster potential as a whole. Because the profiles are usually peaked in the center before flattening further out, the asymptotic dispersions are generally lower than the central dispersions employed above. (These authors also cut the profiles of A2634 and A2666 off at smaller radii, however.) For this reason, we separately plot the asymptotic cluster velocity dispersions, as listed by Fadda et al., against $S_{N}$ in Figure 5-28. The correlation is again strong, with perhaps slightly more scatter (the formal significance is 0.999 ). 
Correlations among cluster richness, dispersion, and X-ray temperature are now well established (e.g., Bahcall 1981; Mushotzsky 1984; Edge \& Stewart 1991; Lubin \& Bahcall 1993; Girardi et al. 1993, 1996) and were to be expected as different measures of the cluster potential (assuming galaxies trace mass). Now, we see a clear relationship between one of these quantities and something apparently unrelated, the GC specific frequency of the central bright galaxy in the cluster. This finding could indicate any of several possibilites. For instance, the efficiency of GC formation for galaxies in denser regions may have been higher, for whatever reason; or, central galaxies in clusters may accrue GCs over time from other cluster members; alternatively, the number of GCs which formed early on scaled with the available mass, but central galaxy luminosity saturated at some maximum threshold, resulting in higher values of $S_{N}$ and the observed independence of BCG luminosity on cluster properties. This final possibility has the aesthetic advantage of combining two separate problems into one. We discuss these issues in $\S 5.3$, where the implications of our results are considered in greater depth.

Figure 5-29 shows that the correlation between $S_{N}$ and galaxy density as measured by Abell galaxy counts (Abell 1958; Struble \& Rood 1987) is much weaker. The high formal significance of the correlation is due to the fact that the lowest $S_{N}$ central galaxies are all in relatively poor clusters; excluding these makes the correlation marginal. However, Abell estimated that the uncertainty in the counts was nearly $20 \%$, and the redshifts of most of the clusters were just photometric guesses when he was doing his counting. Moreover, the number density of galaxies drops steeply out to the $1 r_{A}$ limit, resulting in a large amount of background contaminaton, and making the counts a questionable measure of central density (Beers \& Tonry 1986).

Bahcall (1981) showed that a better measure of the cluster density was provided by background-corrected counts of bright galaxies within $r_{A} / 2$. Those counts correlated better with cluster dispersion and X-ray luminosity than did the Abell counts. However, the present sample has very little overlap with the Bahcall sample; thus, we undertook to do our own counts of bright galaxies within an even smaller radius, set by size of the CCD field, making background correction completely unnecessary. 


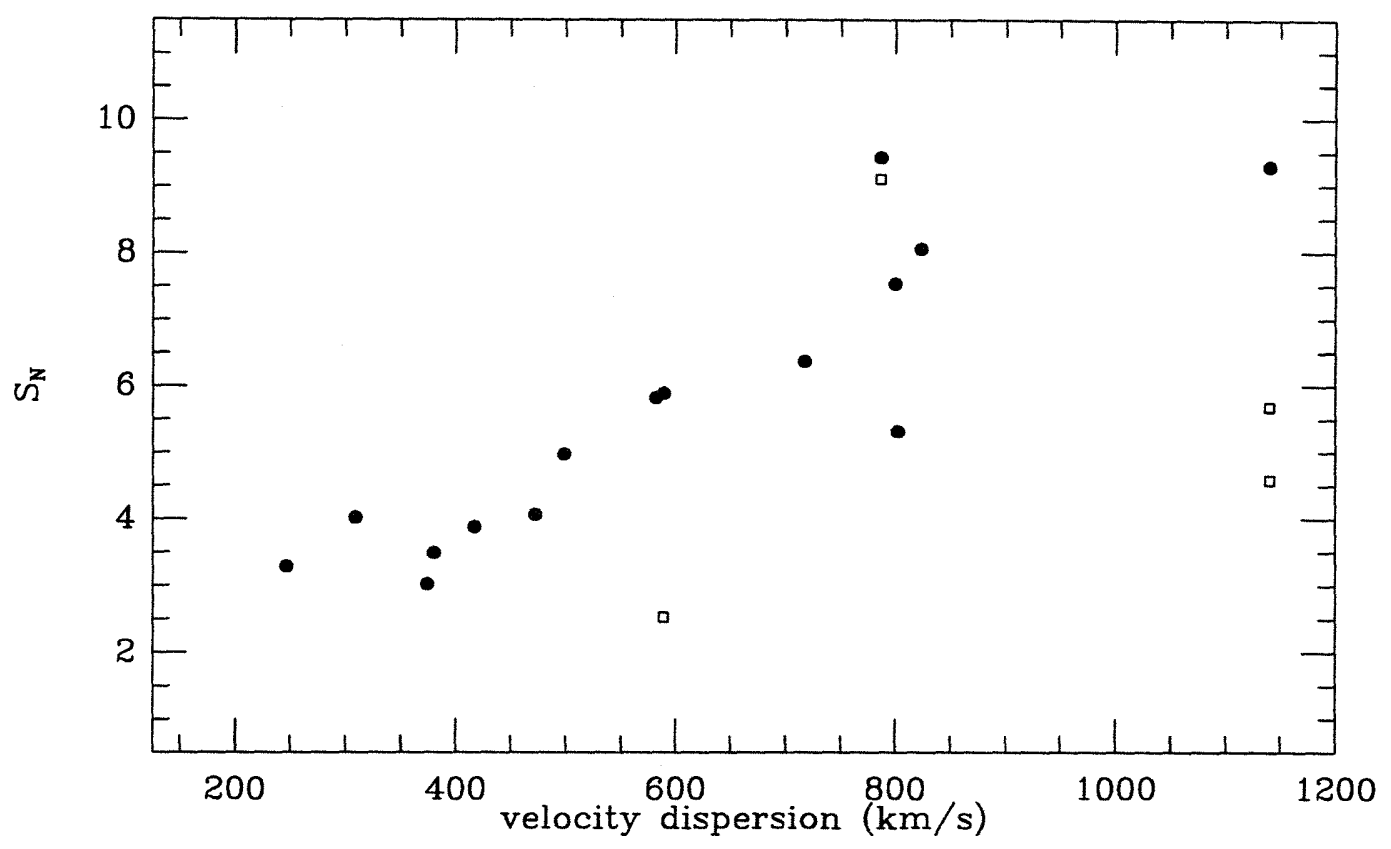

Figure 5-27: The correlation between $S_{N}$ and cluster central velocity dispersion, collected from various sources in the literature. Open squares represent the "secondary" galaxies in clusters with more than one member in the present sample (see text for details); filled circles are rest of the sample.

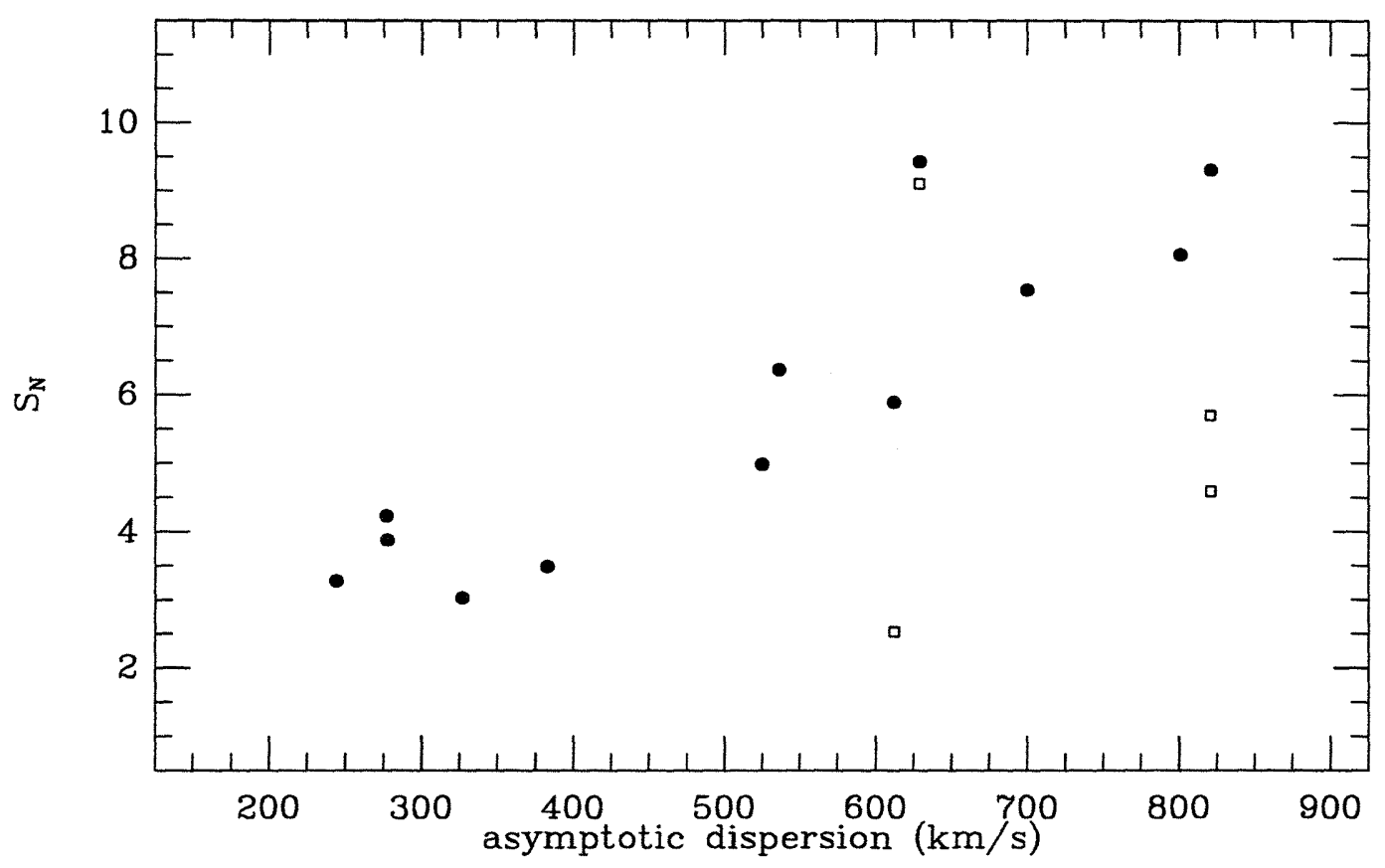

Figure 5-28: The correlation persists, as $S_{N}$ is plotted against the asymptotic velocity dispersions reported by Fadda et al. 1996. Here and in the following figures, symbols are as in Figure 5-27. 


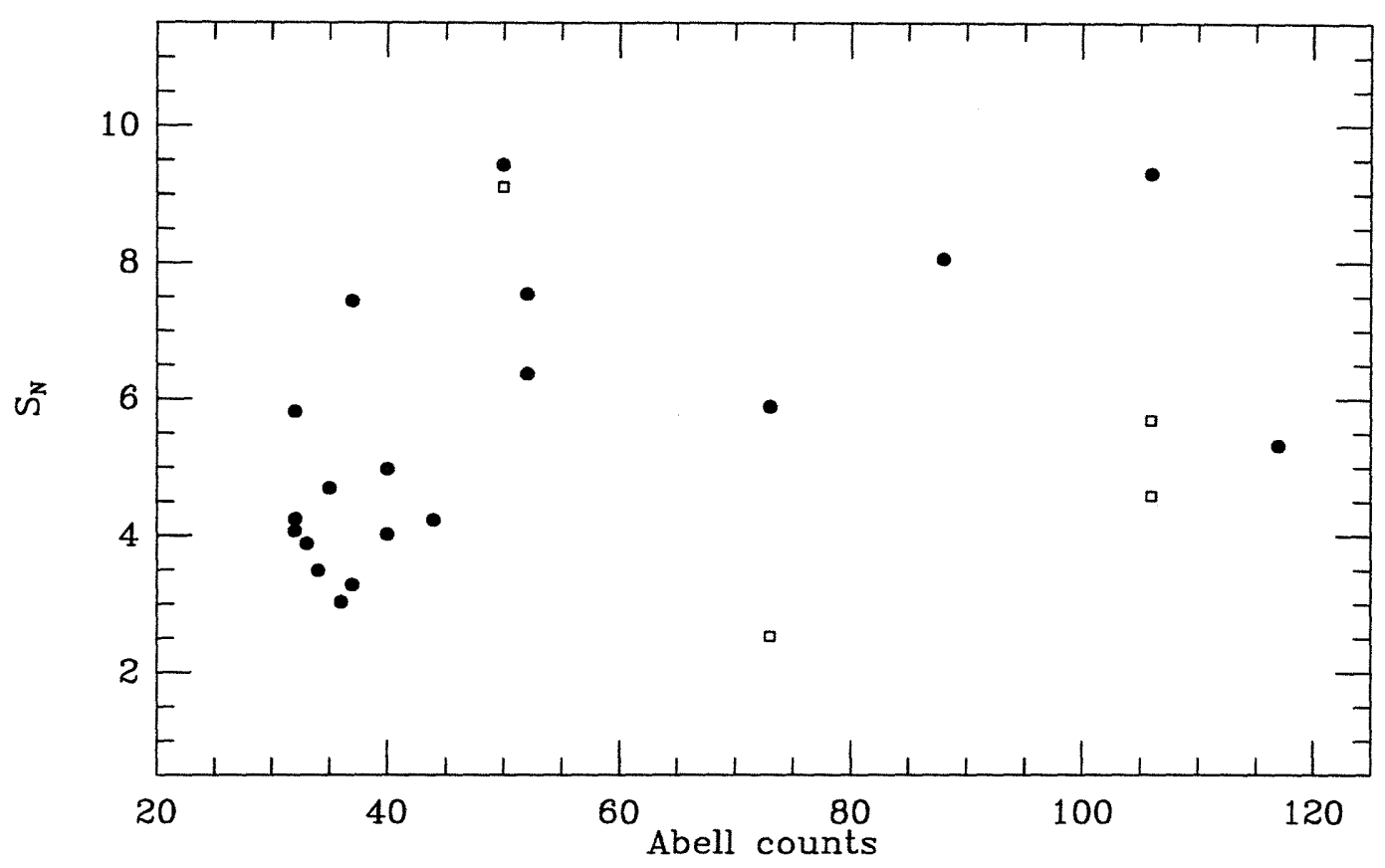

Figure 5-29: $S_{N}$ is plotted against Abell counts, a measure of overall cluster richness.

We counted all galaxies brighter than 0.05 and $0.1 L^{*}$ within several different radial distances from the program galaxy centers, where $L^{*}$ is the characteristic luminosity of the Schechter (1976) function. The value of $L^{*}$ was taken from Lin et al. (1996) and transformed from the $r$-band to our photometric bands following Schneider et al. (1983a). The results of our counts are reported in Table 5.5 which lists the number of neighbors $N_{n}$ brighter than $0.1 L^{*}$ within 32,40 , and $50 h^{-1} \mathrm{kpc}$ and the number brighter than $0.05 L^{*}$ within 32 and $40 h^{-1} \mathrm{kpc}$. Figure 5-30 plots the counts against $S_{N}$. We are hampered by small number statistics, but it is clear that the galaxies surrounded by more neighbors, or in regions of greater galaxy density, tend to have higher $S_{N}$. Again a relationship is suggested between central location within the cluster and $S_{N}$.

Moving on to cluster morphology, we plot $S_{N}$ against Bautz-Morgan type (from Abell et al. 1989) and Rood-Sastry type (Struble \& Rood 1987) in Figure 5-31. There have been reports based on smaller data sets that central galaxy $S_{N}$ correlates with BM type (McLaughlin, Harris, \& Hanes 1993, 1994; Harris, Pritchet, \& Hanes 1995), in the sense of the "later" types (II-III, III) having higher $S_{N}$ central galaxies. The 
Table 5.5: Neighboring Galaxy Counts

\begin{tabular}{r|rrr|cc}
\hline \hline \multicolumn{1}{|c|}{$.10 L^{*}$} & \multicolumn{3}{|c}{$.05 L^{*}$} \\
$r<$ & 32 & 40 & 50 & 32 & $40 \quad\left(h^{-1} \mathrm{kpc}\right)$ \\
Galaxy & $N_{n}$ & $N_{n}$ & $N_{n}$ & $N_{n}$ & $N_{n}$ \\
\hline $\mathrm{A} 262-1$ & 3 & 5 & $\geq 5$ & 5 & 7 \\
$\mathrm{~A} 347-1$ & 0 & 0 & $\geq 0$ & 0 & 0 \\
$\mathrm{~A} 397-1$ & 2 & 2 & 2 & 4 & 4 \\
$\mathrm{~A} 539-1$ & 2 & 4 & 4 & 2 & 4 \\
$\mathrm{~A} 539-2$ & 4 & 6 & 7 & 6 & 8 \\
$\mathrm{~A} 569-1$ & 0 & 1 & 3 & 0 & 1 \\
$\mathrm{~A} 634-1$ & 0 & 1 & 1 & 0 & 1 \\
$\mathrm{~A} 779-1$ & 2 & 3 & 3 & 3 & 4 \\
$\mathrm{~A} 999-1$ & 2 & 4 & 4 & 3 & 6 \\
$\mathrm{~A} 1016-1$ & 1 & 2 & 2 & 1 & 2 \\
$\mathrm{~A} 1177-1$ & 0 & 2 & 2 & 1 & 4 \\
$\mathrm{~A} 1185-1$ & 3 & 3 & 3 & 4 & 5 \\
$\mathrm{~A} 1314-1$ & 0 & 1 & 3 & 1 & 2 \\
$\mathrm{~A} 1367-1$ & 3 & 3 & 3 & 4 & 5 \\
$\mathrm{~A} 1656-1$ & 2 & 4 & 6 & 3 & 5 \\
$\mathrm{~A} 1656-2$ & 5 & 7 & 11 & 5 & 7 \\
$\mathrm{~A} 1656-3$ & 1 & 1 & 2 & 1 & 1 \\
$\mathrm{~A} 2162-1$ & 0 & 1 & 1 & 0 & 3 \\
$\mathrm{~A} 2197-1$ & 0 & 1 & 1 & 0 & 1 \\
$\mathrm{~A} 2197-2$ & 1 & 1 & 2 & 2 & 2 \\
$\mathrm{~A} 2199-1$ & 5 & 5 & 8 & 6 & 7 \\
$\mathrm{~A} 2634-1$ & 2 & 3 & 3 & 3 & 4 \\
$\mathrm{~A} 2666-1$ & 1 & 1 & 3 & 1 & 1 \\
\hline \hline
\end{tabular}



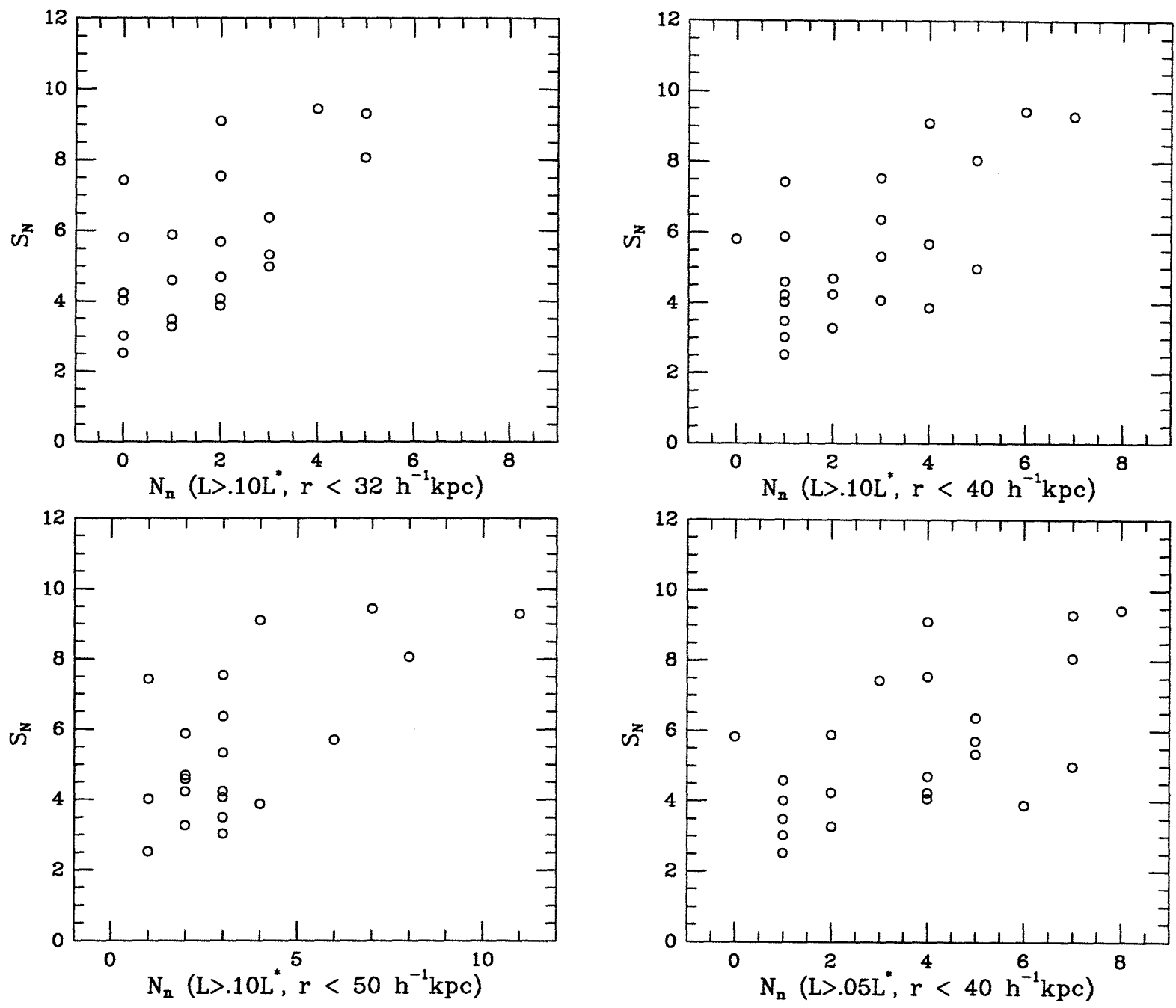

Figure 5-30: $S_{N}$ is plotted against the total number of neighboring galaxies brighter than $0.1 L^{*}$ within 32,40 , and $50 h^{-1} \mathrm{kpc}$ of the BCG/SRG center, and against the number of neighbors brighter than $0.05 L^{*}$ within $40 h^{-1} \mathrm{kpc}$. 
classification in this system is based on the relative dominance of the central galaxy in the cluster, with BM I clusters being dominated by single giant galaxies, and BM III cluster having no clearly dominant galaxy. McLaughlin et al. (1993) first suggested that the apparent anti-correlation of $S_{N}$ with central galaxy dominance was due to dynamical evolution, with the central giants in the BM I and I-II clusters having diluted their originally high- $S_{N}$ down to lower levels through repeated mergers with other galaxies in the cluster. It was an interesting suggestion, but we do see not see a correlation of $S_{N}$ with BM type in this larger, more homogenous data set. In particular, we find no evidence that such $S_{N}$ dilution is taking place.

Rood-Sastry type is also usually thought of in evolutionary terms, with the evolutionary state becoming more advanced along the following morphological sequence: Irregular, Flattened, Core-dominated, Linear, Binary, and cD. Again, our immediate reaction is that no correlation exists between central galaxy $S_{N}$ and RS type. However, Schombert \& West (1990) suggested that this classifications system reflected dynamical evolution only up to the $\mathrm{L}$ class, with the $\mathrm{B}$ and $\mathrm{cD}$ classes (clusters dominated by two giant galaxies and one giant galaxy, respectively) representing further evolution of the galaxy luminosity function following cluster virialization. This suggestion was based on an apparent correlation of the I-F-C-L sequence with supercluster environment. It is conceivable that there is a systematic change of $S_{N}$ along this subsequence of the RS system, but if so, it is most likely an ancillary consequence of the dependence central galaxy $S_{N}$ on cluster density, seen most clearly in the correlations with velocity dispersion (above) and X-ray properties (below).

From the correlation of $S_{N}$ with cluster velocity dispersion found in Figure 5-27, and the known relation between cluster velocity dispersion and the temperature of the X-ray emitting gas (Lubin \& Bahcall 1993; Figure 5-37, below), we expect some correlation between $S_{N}$ and cluster X-ray properties. Figures 5-32 and 5-33 show $S_{N}$ plotted against the logarithms of the cluster X-ray temperature $T_{X}(\mathrm{keV})$ and X-ray luminosity $L_{X}$ in the $0.5-4.5 \mathrm{keV}$ band $\left(\mathrm{ergs} \mathrm{sec}^{-1}\right.$ ) from Jones \& Forman (1996). While previous investigations found no correlations of $S_{N}$ with these properties (Harris et al. 1995; West et al. 1995), our larger, more homogeneous data set clearly shows 

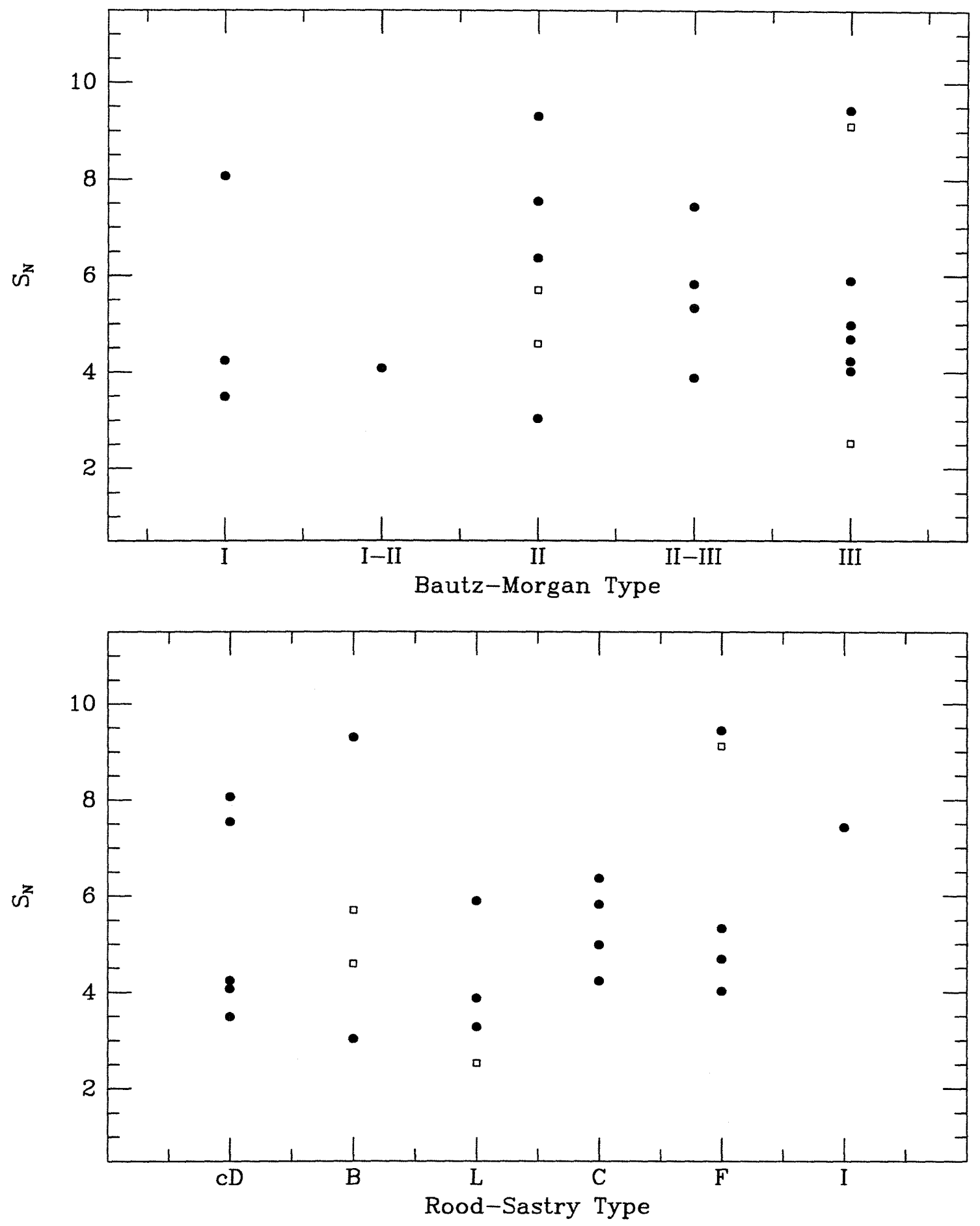

Figure 5-31: $S_{N}$ is plotted against morphological type in the Bautz-Morgan and Rood-Sastry classification systems. Unlike previous investigations based on many fewer clusters, we find no evidence of any correlation in these data. Symbols are as in Figure 5-27. 
that there is a correlation, with significance level of $\sim 0.998$.

The scatter in these plots is larger than in the velocity dispersion plots, but that is at least partially due to the larger uncertainties in the X-ray temperatures. If the observed variation of $S_{N}$ among these galaxies is driven by environmental density, then $S_{N}$ should correlate more strongly with $T_{X}$ than with $L_{X}$ because, assuming hydrostaic equilibrium, the gas temperature is determined simply by the depth of the cluster gravitational potential while the luminosity also depends on the amount of gas present. These two plots look so similar because half of the temperatures were estimated from the cluster $L_{X}-T_{X}$ relation (see Table 4.2), and there will be definite scatter about this relation for the reason just stated. These estimated temperatures have $1 \sigma$ uncertainties of about $20 \%$ (Jones 1996), while the uncertainties in the velocity dispersions used above are $\lesssim 10 \%$.

Recently, West et al. (1995) have discussed the implications of possible "intracluster globular clusters" (IGCs) which belong to the cluster as a whole, not to individual galaxies. Like the hot intracluster gas, the hypothesized IGCs trace the gravitational potential. If a giant galaxy happens to lie near the cluster center, it will appear to have a large value of $S_{N}$ due to these "excess" GCs which have become ipso facto associated with it. West et al. define the excess to be the total number of GCs which elevate the galaxy's $S_{N}$ above a value of 4 . They predict that the observed GC excess should correlate directly with the projected cluster mass density at the radial position $r$ of the galaxy from the cluster X-ray center. Assuming hydrostatic equilibrium and an isothermal potential, this mass density will be proportional to $T_{X} /\left(1+r^{2} / r_{c}^{2}\right)$, where $r_{c}$ is the core radius of the cluster.

In Figure 5-34, we plot the GC excess, defined relative to $S_{N}=3.5$, which more accurately reflects the lower $S_{N}$ limit for our sample, against $T_{X} /\left(1+r^{2} / r_{c}^{2}\right)$, where the $r_{c}$ values come from Jones \& Forman. In support of the West et al. predictions, we find that there does appear to be a roughly linear relationship. (Our GC excess numbers are systematically lower than in the corresponding West et al. plot because these are derived from our "metric" $S_{N}$ values and the magnitudes shown in Figure 524.) Even the non-central galaxies (open symbols) should follow this relationship, 
since it takes into account galaxy position in the cluster, and these galaxies do appear to deviate less than in the previous two plots.

Figure 5-35 shows what happens to the correlation seen in Figure 5-34 if the same core radius of $30 h^{-1} \mathrm{kpc}$ is used for all the clusters. This was the approximation made by West et al. in their reported correlation. The one improvement is that A1656-1 moves to the left, but otherwise, the true core radii for these clusters, ranging from 45 to $215 \mathrm{~h}^{-1} \mathrm{kpc}$, result in a much better correlation (a rank order coefficient of 0.68 instead of 0.52 , indicating a significance level of 0.998 instead of 0.981 ).

In Figure 5-36, we plot $S_{N}$ against "local X-ray luminosity" $L_{X} /\left(1+r^{2} / r_{c}^{2}\right)$, showing that the correlation seen in Figure 5-33 can be improved in this way, using the correct core radii. The secondary cluster galaxies move into better alignment and the scatter among the lower $S_{N}$ galaxies decreases somewhat.

Finally, for the edification of the reader, Figure 5-37 displays the correlation between velocity dispersion and X-ray temperature in these clusters. The weighted least squares fit drawn through the points indicates that the velocity dispersion scales with temperature as $v_{R M S}=363 \pm 53\left(k T_{X}\right)^{0.55 \pm .07} \mathrm{~km} \mathrm{~s}^{-1}$, where $k T_{X}$ is in units of $\mathrm{keV}$. This relation is consistent with both the gas and the galaxies being in hydrostatic equilibrium within isothermal cluster potentials. Our fit results agree with the those of Lubin \& Bahcall (1993), who found $v_{R M S}=332 \pm 52\left(k T_{X}\right)^{0.6 \pm .1} \mathrm{~km} \mathrm{~s}^{-1}$ for a cluster sample three times larger than this one, and Girardi et al. (1996), who obtained $v_{R M S}=363 \pm 25\left(k T_{X}\right)^{0.56 \pm .05} \mathrm{~km} \mathrm{~s}^{-1}$, in remarkable agreement with our fit, even though they used asymptotic dispersions and a more sophisticated fitting procedure.

In the final section of this chapter, we will discuss the implications of our data for the various theoretical models. We then describe how the correlations found here improve our understanding of GC systems, and the mechanisms which effect $S_{N}$ in Abell cluster central galaxies. Before doing that, however, we pause for an interlude on the choice of reference frame. 


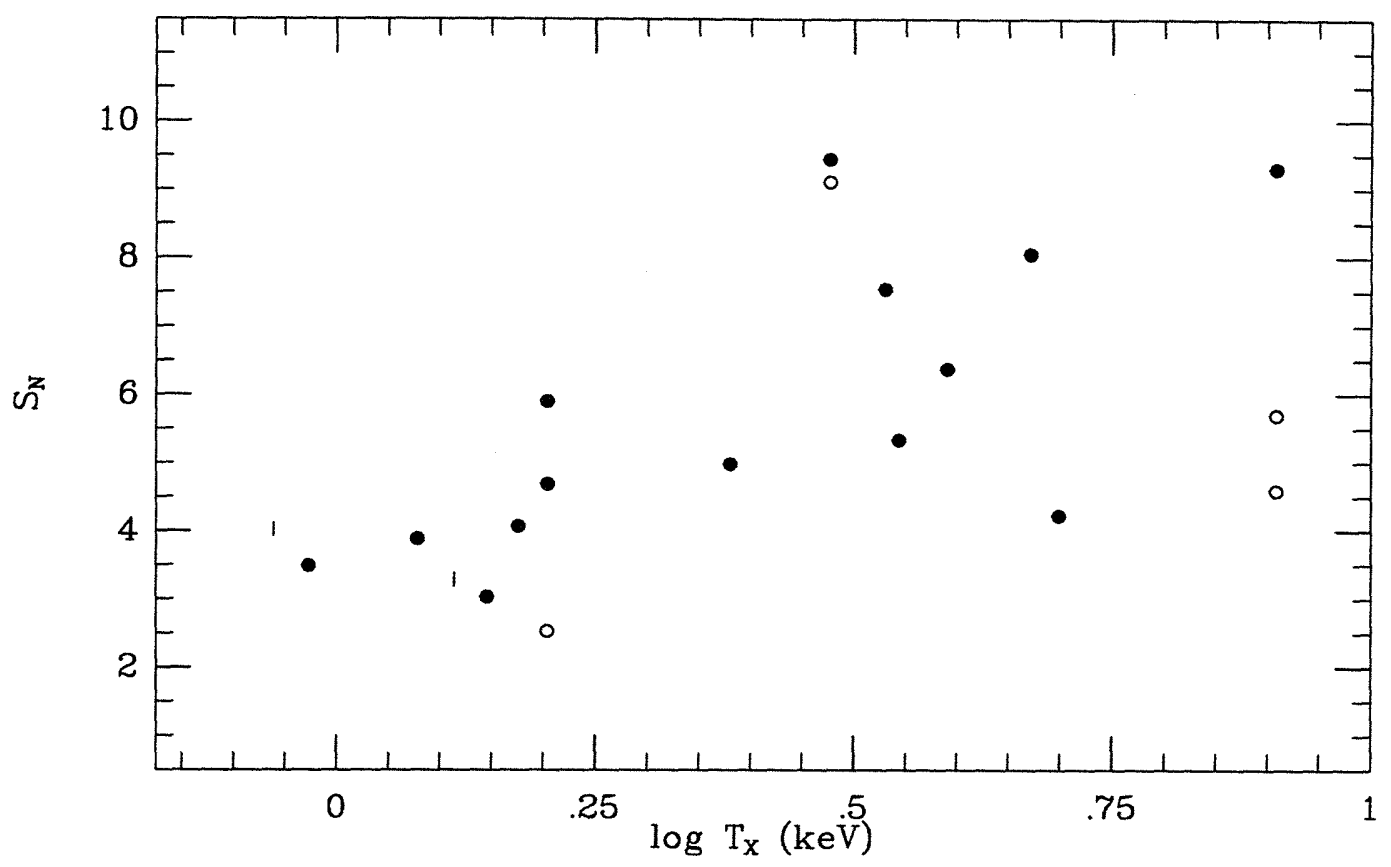

Figure 5-32: $S_{N}$ versus temperature of the X-ray emitting intracluster gas. Filled circles represent the BCGs in clusters with only one member in this sample or the more central galaxies in clusters with more than one. Open circles represent the less central (or "secondary") galaxies; short vertical lines represent galaxies in cluster with only an upper limit on their X-ray emission.

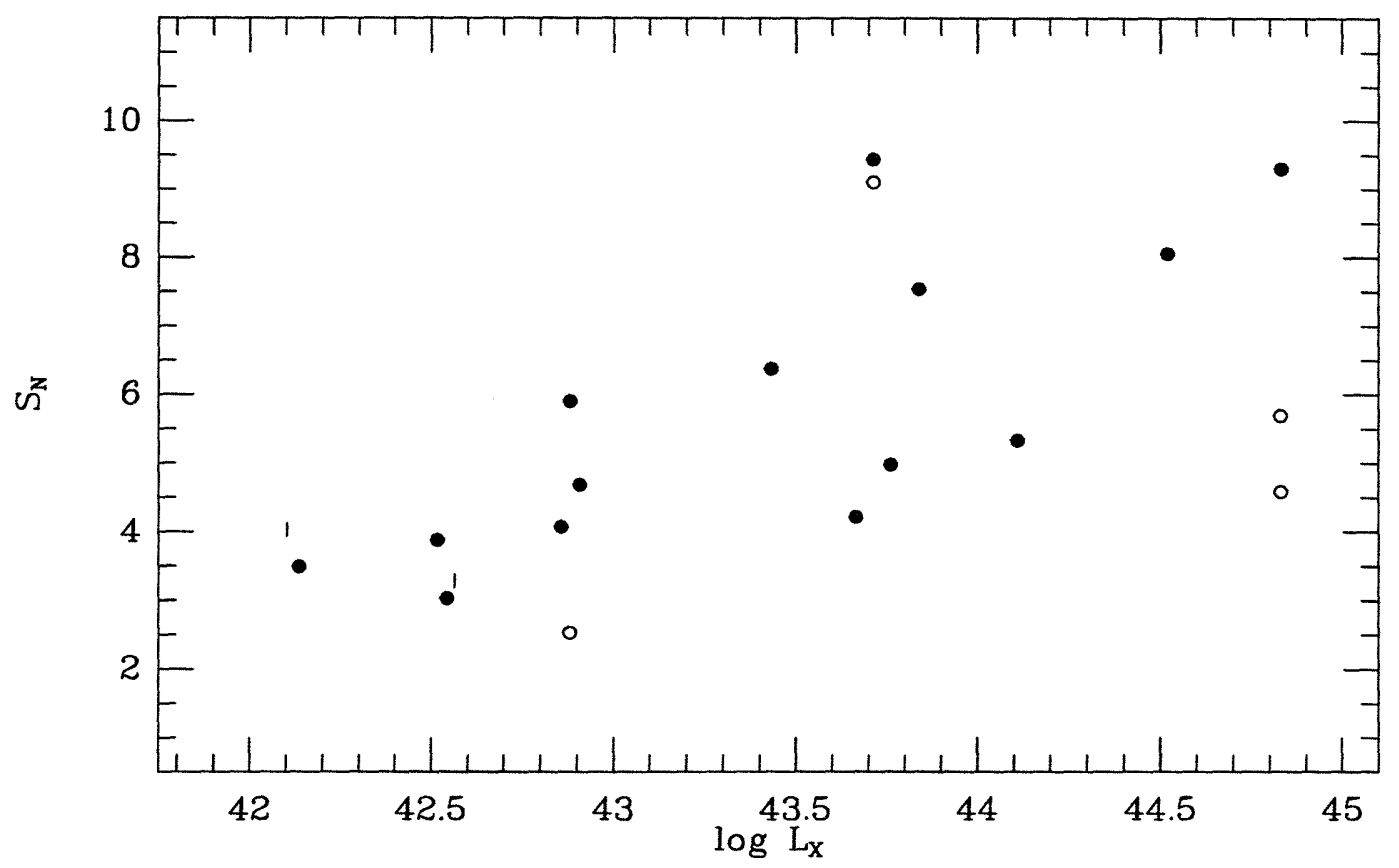

Figure 5-33: $S_{N}$ versus total X-ray luminosity within $2 h^{-1} \mathrm{Mpc}$ of the cluster X-ray center. Here and in the following figures, the symbols are as in Figure 5-32. 


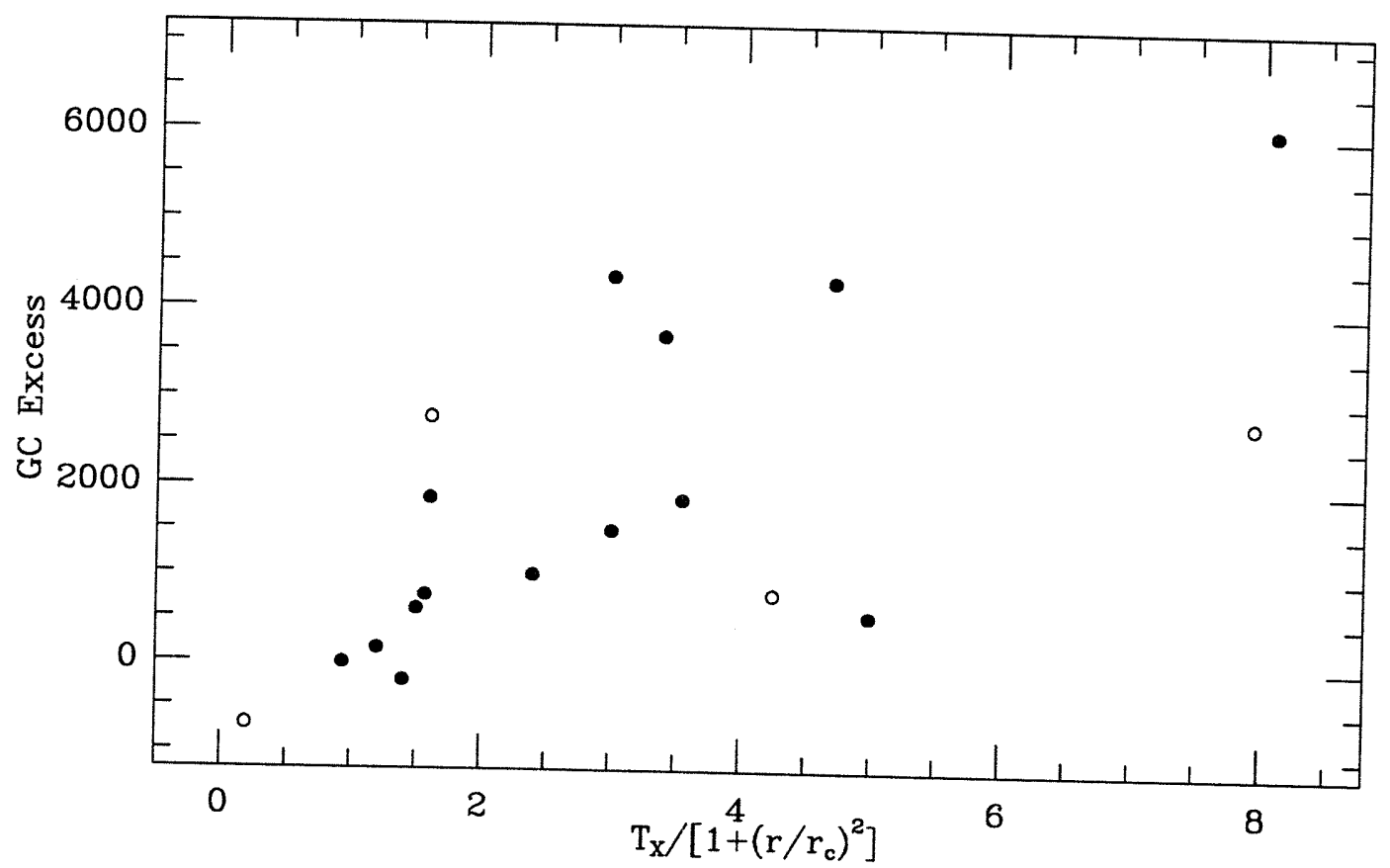

Figure 5-34: The observed "excess" number of GCs in the IGC model is plotted against a quantity proportional to the projected matter density of the cluster at the radial position of the galaxy.

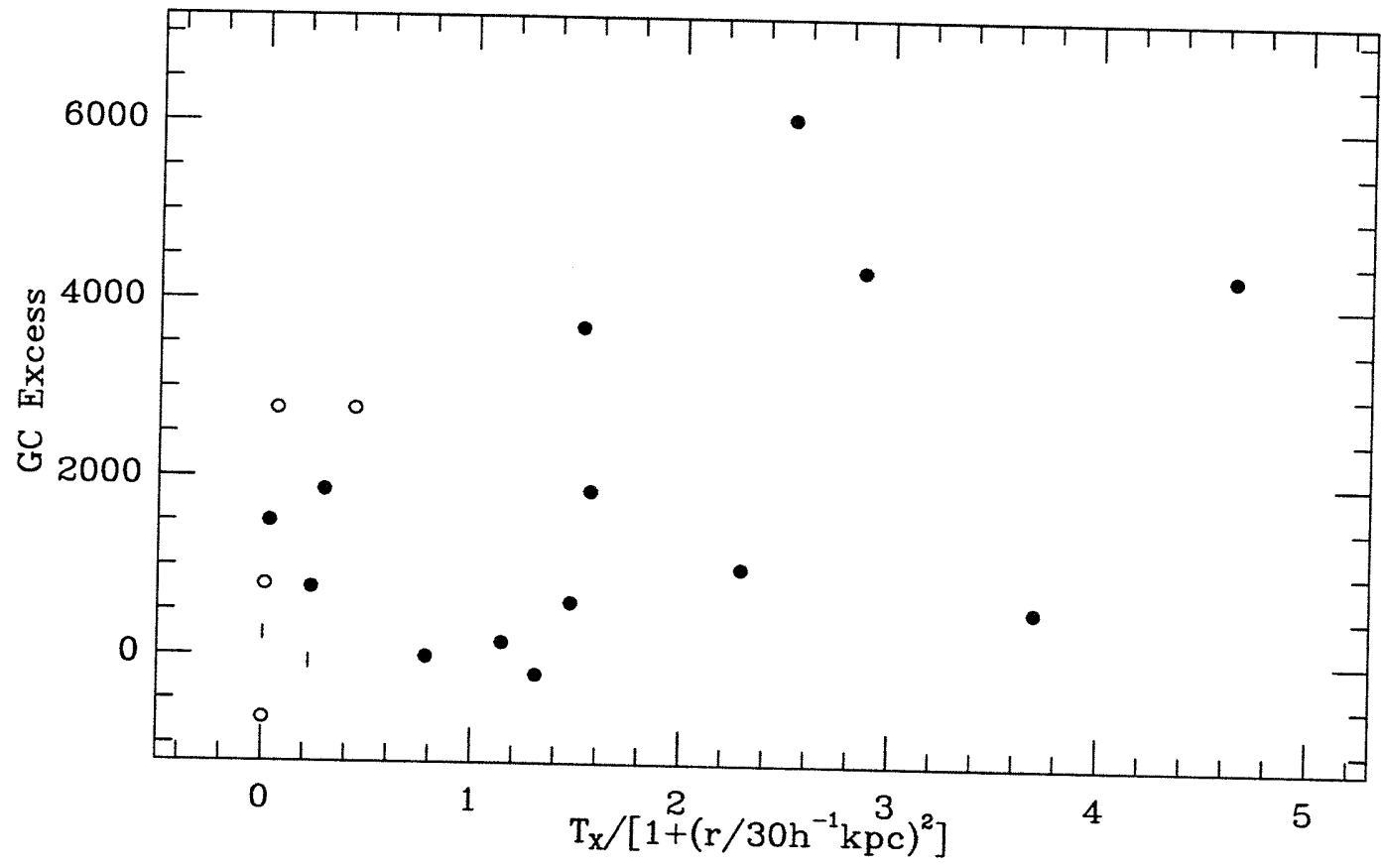

Figure 5-35: Same as Figure 5-34, but here a single core radius of $r_{c}=30 h^{-1} \mathrm{kpc}$ is used for all the galaxy clusters, as in West et al. The correlation seen in Figure 5-34 worsens with this approximation. 


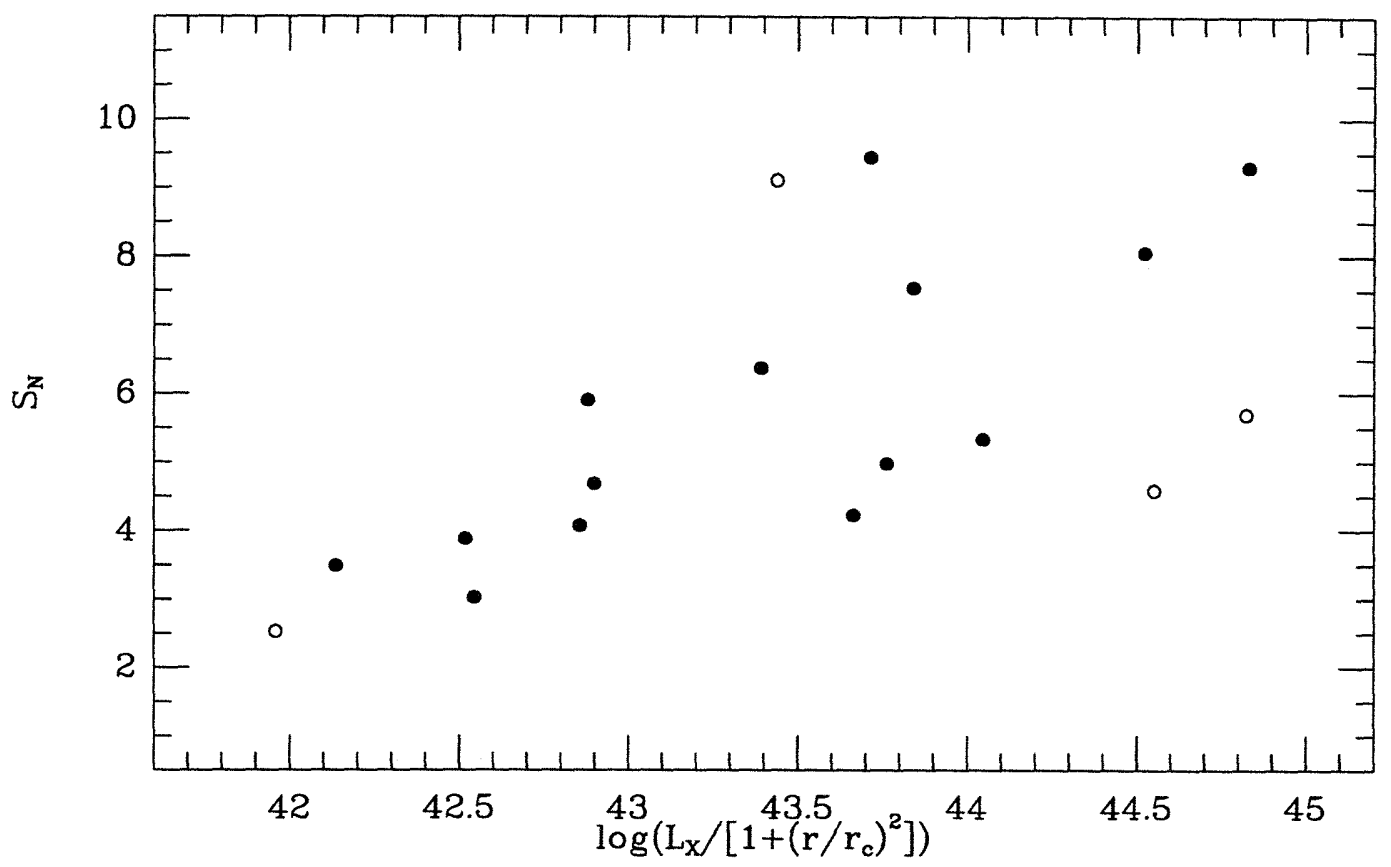

Figure 5-36: $S_{N}$ is shown plotted against the radially weighted, or "local", X-ray luminosity at the position of the galaxy. The correlation observed in Figure 5-33 improves with this weighting.

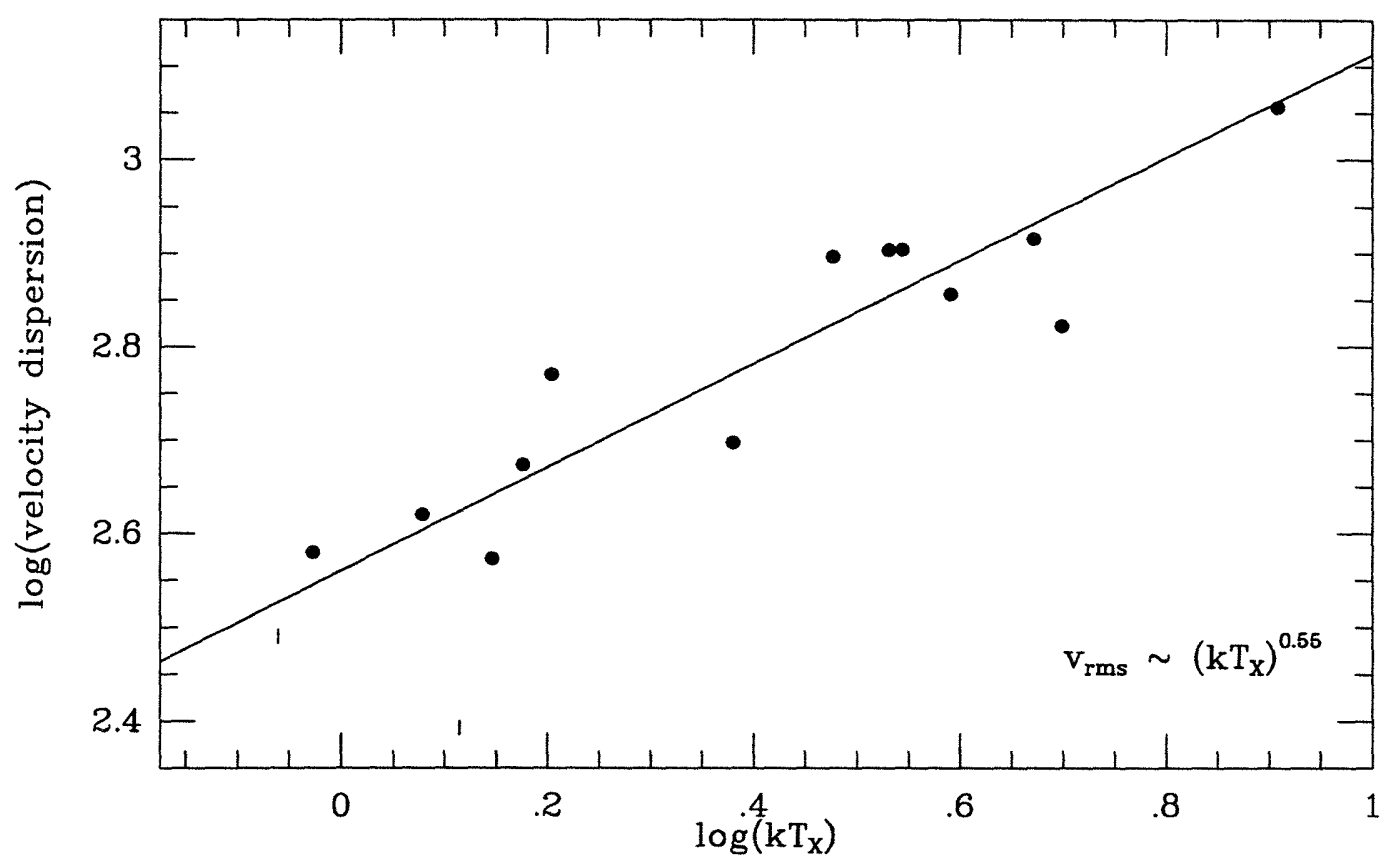

Figure 5-37: The velocity dispersion of the cluster galaxies $\left(\mathrm{km} \mathrm{s}^{-1}\right)$ is plotted against cluster X-ray temperature (keV), in logarithmic units. The solid line represents a fit to the relation. The two clusters with only upper limits on $T_{X}$ are plotted as short vertical lines but were not used in the fit. 


\subsubsection{The Abell Cluster Inertial Frame}

The values of $S_{N}$ and GCLF width $\sigma$ reported and discussed in previous sections of this chapter were all calculated in the CMB frame, as stated at the outset. However, there is another relevant frame which might also have been adopted, the Abell Cluster Inertial (ACI) frame. The ACI frame is defined to be at rest with respect to the largescale Abell cluster bulk flow discovered by LP using the $L_{m}-\alpha$ distance indicator.

In fact, the analysis was done twice simultaneously, using both $\mathrm{CMB}$ and ACI frame velocities to estimate $m^{0}$ and galaxy luminosities. In both analyses, the Virgo distance modulus was set to the Cepheid result of 31.02, and Virgo was placed into each of these frames using the $3.71 \pm 0.10$ relative Coma-Virgo distance modulus reported by van den Bergh (1992). This combination corresponds to $H_{0}=82 \mathrm{~km} \mathrm{~s}^{-1}$ $\mathrm{Mpc}^{-1}$ for the CMB frame and $H_{0}=75 \mathrm{~km} \mathrm{~s}^{-1} \mathrm{Mpc}^{-1}$ for the ACI frame, but the important aspect is that the relative distances between the galaxies change as a function of galactic coordinates, according to the transformation given by LP.

Table 5.6 lists the $S_{N}$ and $\sigma$ results of our analysis in the ACI frame. The values were derived in an identical manner to those presented in Table 5.3, but the ACI frame velocities were used instead of CMB velocities. The values of $S_{N}$ are generally higher. This is a consequence of the fact that the Coma-Virgo distance is fixed, but the velocity of Coma is nearly $10 \%$ smaller in the ACI frame (see Table 4.2).

Among the galaxies in clusters without fixed distances (i.e., everything but Coma), the fractional scatter in $S_{N}$ increases by $5 \%$ (and the absolute scatter increases by $11 \%$ ) when the ACI frame is used instead of the CMB frame. In addition, the mean and median GCLF $\sigma$ values among those with errors smaller than 0.15 mag are now $\langle\sigma\rangle=1.48 \mathrm{mag}$ and $\sigma_{m e d}=1.51 \mathrm{mag}$, respectively; dropping the "fixed point" Coma galaxies, these values increase to 1.51 and $1.53 \mathrm{mag}$. (See Figure 5-38.) Thus, unlike the results for $\sigma$ in the CMB frame, which were discussed in $\$ 5.2 .1$, we do not find good agreement between the values of $\sigma$ in the ACI frame and the $\sigma=1.40$ mag width of the GCLF $m^{0}$ calibrator M87. For these reasons, we decided to concentrate the rest of the analysis on the results found using CMB frame velocities, as we have done in the preceding sections and in the discussion below. 
Table 5.6: Metric Specific Frequencies and GCLF Widths in ACI Frame

\begin{tabular}{|c|c|c|c|c|c|}
\hline Galaxy & $S_{N} \pm$ & $\sigma_{\mathrm{LF}} \pm$ & Galaxy & $S_{N} \pm$ & $\sigma_{\mathrm{LF}} \pm$ \\
\hline A $262-1$ & $5.4_{1.5}^{1.8}$ & $1.53: .14$ & A1314-1 & $4.3_{1.0}^{1.2}$ & 1.62 .21 \\
\hline A $347-1$ & $6.3_{1.5}^{1.8}$ & $1.48: .10$ & A1367-1 & $5.3_{1.1}^{1.4}$ & $1.53 . .11$ \\
\hline A397-1 & $5.2_{1.3}^{1.7}$ & 1.53 .11 & A1656-1 & $5.7_{1.3}^{1.3}$ & 1.37 .12 \\
\hline A539-1 & $10.1_{2.9}^{3.5}$ & 1.51 .13 & A1656-2 & $9.3_{2.0}^{2.0}$ & 1.43 .09 \\
\hline A539-2 & $10.5_{2.8}^{3.5}$ & 1.55 .12 & A1656-3 & $4.6_{1.3}^{1.5}$ & $1.33 . .11$ \\
\hline A569-1 & $3.3_{1.0}^{1.2}$ & 1.50 .24 & A2162-1 & $7.5_{1.9}^{2.3}$ & 1.74 .16 \\
\hline A634-1 & $4.1_{1.1}^{1.3}$ & 1.81 .18 & A2197-1 & $2.6_{1.3}^{1.4}$ & $1.56 . .30$ \\
\hline A $779-1$ & $4.1_{0.9}^{1.0}$ & 1.39 .12 & A2197-2 & $5.9_{1.2}^{1.5}$ & 1.51 .11 \\
\hline A999-1 & $4.0_{1.4}^{1.6}$ & 1.59 .24 & A2199-1 & $8.2_{1.9}^{2.4}$ & 1.55 .09 \\
\hline A1016-1 & $3.3_{1.1}^{1.2}$ & $1.86 . .23$ & A2634-1 & $8.1_{1.9}^{2.4}$ & 1.61 .100 \\
\hline A1177-1 & $4.3_{1.1}^{1.3}$ & 1.51 .18 & A2666-1 & $3.8_{1.1}^{1.3}$ & $1.53 . .23$ \\
\hline A1185-1 & $6.5_{1.5}^{1.9}$ & $1.35: 10$ & & & \\
\hline
\end{tabular}

Note: Same as Table 5.3 but all calculations done in the ACI frame.

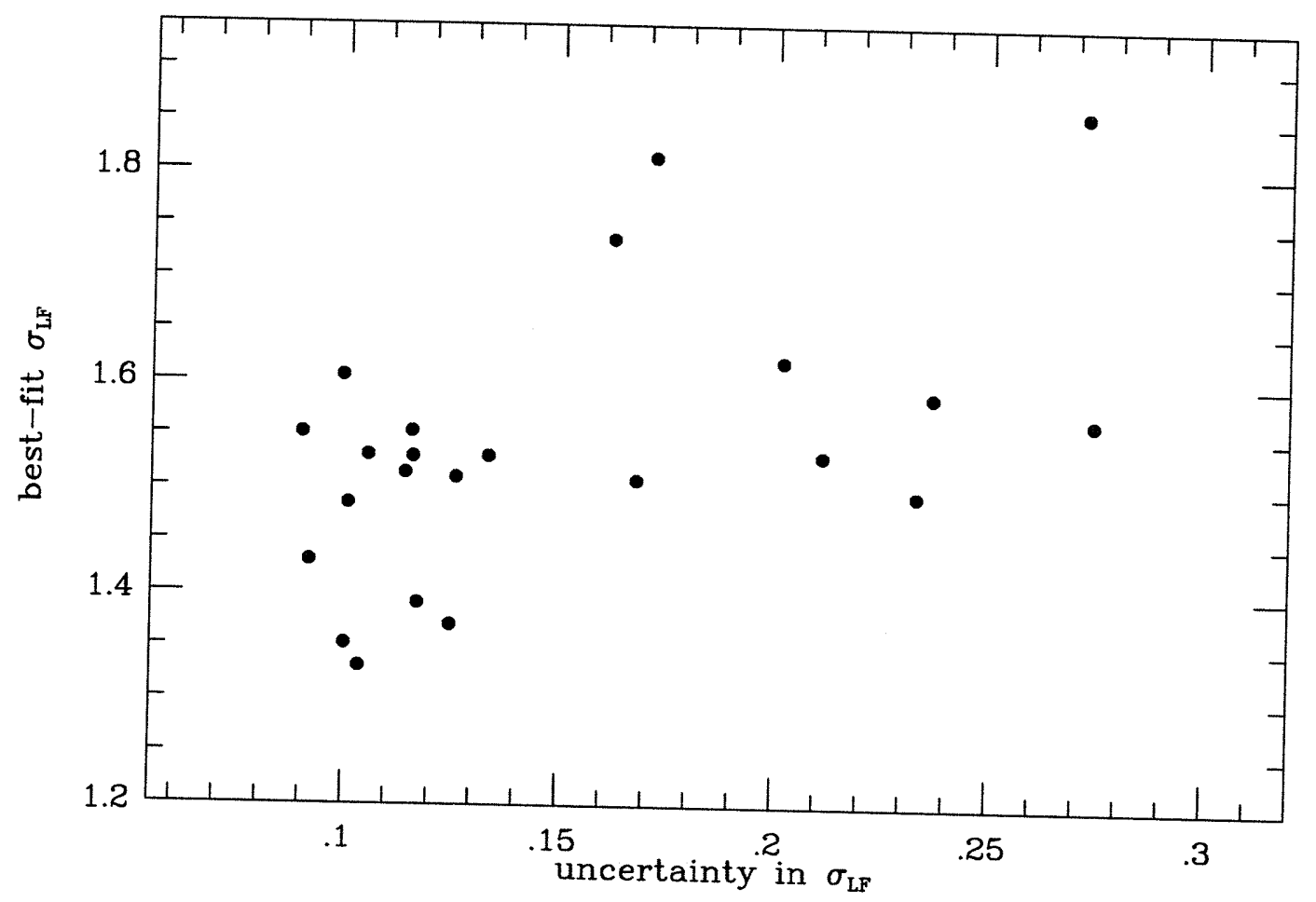

Figure 5-38: The derived GCLF width $\sigma$ in the ACI frame is plotted against its uncertainty. Again, the more uncertain values tend to be high, but here the median of the well-determined values is also high compared to the CMB frame results. 


\subsection{Discussion}

\subsubsection{Are High- $S_{N}$ BCGs Special?}

Among the bright cluster galaxies in this sample, $S_{N}$ varies continously as a function of certain well-defined galaxy and cluster parameters. Thus, the answer to the question posed above is a decided "no", in the sense that they do not constitute a special class of object, but rather occupy the high end of the $S_{N}$ distribution. However, the primary factor governing $S_{N}$ is not galaxy luminosity as suggested by Djorgovski \& Santiago (1992). Rather, the total number of GCs appears to be determined primarily by cluster environmental factors, unlike the central galaxy luminosity, which is remarkably immune to variations in cluster properties (e.g., Hoessel, Gunn, \& Thuan 1980; PL).

One is prompted to ask whether or not the results presented here conflict with the prevailing idea of "normal" and "anomalous" $S_{N}$ systems (e.g., Harris 1986; Pritchet $\&$ Harris 1990; Harris et al. 1995). Before proceeding, we note that the primary review on this topic (Harris 1991) stated, "whether [high- $S_{N}$ galaxies] represent just the upper end of a continuum of $S_{N}$ values or if they are truly distinct remains unclear." We begin by considering the prototypical high- $S_{N}$ galaxy M87. The most recent determination of $S_{N}$ for this galaxy was by McLaughlin et al. 1994, who reported a global value of $S_{N}=14.3 \pm 1.0$, before taking into account uncertainties in the distance or GCLF. Those authors adopted the same distance as we have; however, their fitted GCLF parameters significantly disagree with those determined with HST (Whitmore et al. 1995). This is understandable, as the fitted GCLF width and turnover are strongly correlated when the data do not go significantly fainter than $m^{0}$.

The large $14^{\prime} \times 14^{\prime}$ field of the McLaughlin et al. data makes it superior to HST for deriving $S_{N}$, and they provided a convenient correction factor, which is a function of the GCLF parameters and distance modulus and can be applied to their quoted $S_{N}$. Plugging the HST GCLF results into their correction formula, we find for a global value $S_{N}(M 87)=11 \pm 1$. Moreover, $S_{N}$ increases with radius in M87, so its "metric $S_{N} "$, as we report for our sample galaxies, should be smaller than this. Thus, given 
the uncertainties, including the systematic error in the calibration of Virgo to the CMB frame, we can only conclude that M87 is at the high end of the continuous distribution in $S_{N}$ which we have observed. M87 may prove enormously important as a clue to the variation of central galaxy $S_{N}$ with cluster properties.

The other well-studied galaxy that has been numbered among the high $S_{N}$ systems is the Fornax cD NGC 1399. This galaxy was first reported to have $S_{N} \sim 16$ (Bridges, Hanes, \& Harris 1991). A more recent study, using GCLF parameter which agree with ours from Chapter 3, found $S_{N}=12 \pm 3$ (Kissler-Patig et al. 1996). However, this later study used a distance modulus that was 0.30 mag smaller than the new HST Cepheid result for this cluster (Silberman et al. 1996). Using the Cepheid distance modulus, $S_{N}$ for this galaxy becomes $S_{N}(\mathrm{~N} 1399)=9 \pm 2$. The GCs and halo light follow similar distributions, so the metric $S_{N}$ would not differ much from the global value. This lies in the range of the galaxies studied here, though higher than we would expect for a cluster of low dispersion. On the other hand, as pointed out previously, Fornax is not a rich cluster (though it is very compact) so it is not clear how the $S_{N}$ of its central galaxy should compare to those studied here.

The rest of the high $S_{N}$ values found in the literature generally scale with the $S_{N}$ of M87. For instance, Harris et al. (1995) find $22 \pm 7$ and $13 \pm 6$ for the central cD galaxies in A2052 and A2107 (both outside the redshift limit of our survey; their other cluster, A2666, is discussed below). These numbers were derived relative to an assumed M87 value of 15; using the value of 11 implied by the HST GCLF yields $S_{N}(\mathrm{~A} 2052)=16 \pm 5$ and $S_{N}(\mathrm{~A} 2107)=9.5 \pm 4.4$, which are both still high, but not even the A2052 $S_{N}$ can be called anomalous, given the uncertainties.

Direct comparisons between our results and literature values are possible for several galaxies. In Chapter 2 we mentioned that our global values of $S_{N}$ were in agreement with those found by Harris $(1987,1991)$, but in this chapter we revised those numbers down based on better (RC3) photometry. In fact, Harris also used RC2 photometry, so his $S_{N}$ numbers get revised down in an identical manner; in particular, his value for NGC 4874 becomes $9 \pm 4.5$.

Butterworth \& Harris studied A1367-1 and reported the ratio $S_{N}(\mathrm{~A} 1367) / S_{N}(\mathrm{M} 87)$ 
as a function of radius (it varied from about 0.4 to 0.55 ). Reading the value of this ratio at our metric radius of $32 h^{-1} \mathrm{kpc}$, or $\sim 1^{\prime} .8$, and using the M87 $S_{N}$ from above gives $S_{N}=5.2 \pm 1$, very close to our value; and even if we had used the larger $S_{N}$ of 14 for M87, we would agree with their results within the errors.

For A2666-1, Harris et al. (1995) find $S_{N}=3 \pm 2$, again assuming the $S_{N}$ of 15 for M87. Using the smaller M87 value tendered in this discussion, their value drops to $\sim 2.2$. In either case, it is close to our value of 3.5 for this galaxy. Finally, for the A2199 cD NGC 6166, Pritchet \& Harris (1990) reported $S_{N} \lesssim 4$, but a redetermination based on improved data found $S_{N}=9_{-4}^{+9}$ (Bridges, Carter, Harris, \& Pritchet 1996). The latter result agrees well with our value of 8.1 for this galaxy.

In summary, for the few cases in which direct comparisons are possible, our results are in good agreement with published values. However, we believe that our larger, more homogenous data set affords a clearer view of the overall picture. We find that there is a continuum of possible $S_{N}$ values for bright cluster galaxies. Where a galaxy falls in this continuum may even be predictable from the cluster velocity dispersion, or $T_{X}$, and the projected distance of the galaxy from the cluster center.

Several models have been proposed to explain the higher $S_{N}$ values of central galaxies in clusters. Although the results reported here do not support the idea of a clear dichotomy between "normal" and "high- $S_{N}$ " systems, the models are based on density considerations and may therefore shed some light on the correlations found above; alternatively, the observed correlations may be able to rule out some of the theories. The following section reviews the models in the light of our results. The final section then attempts to synthesize a coherent picture of GCs around central galaxies in clusters.

\subsubsection{How Do the Models Fare?}

Theories of globular cluster formation have a long history, dating back at least to the work of Peebles \& Dicke (1968). We cannot review the entirety of it; nor can our results illuminate such things as metallicity gradients or the dark past of the Milky Way's outer halo, for instance. We can, however, say a great deal about the variation 
of $S_{N}$ among central galaxies in rich clusters, much more than hitherto possible. Thus, we consider in turn only those models which attempt to explain the wide variation of $S_{N}$ among central galaxies in clusters. We have attempted to logically distinguish among the models, but there is some overlap, as will be seen.

(1) Initial conditions. Until now, it was believed that, "No obvious correlation exists between $S_{N}$ and any intrinsic property of either the BCG or the galaxy cluster in which it resides" (West et al. 1995). This statement was based largely on the conclusions of Harris et al. (1995), who found no correlation of $S_{N}$ with cluster X-ray properties in a data set composed of their three galaxies and others assembled from the literature, including galaxies in 4 more Abell clusters, 3 AWM and MKW poor clusters, Fornax, and Virgo. Harris et al. interpreted their results as indicating that GC formation in central cluster galaxies occurred early on, and reflected local initial conditions, thus the lack of any correlations.

This view, that modern galaxies with high values of $S_{N}$ "were special ab initio" and consequently formed their GCs "superefficiently", we call the "initial conditions" scenario. It goes back to Harris (1981) and has been the prevailing view in the literature (e.g., the review by Harris 1991) since van den Bergh (1984) showed that it was the one favored on balance by the available data. Some of the most strenuous recent arguments in its favor have been by McLaughlin, Harris, \& Hanes $(1993,1994)$, based on the incipient $\mathrm{cD}$ halo of M87. These authors also proposed central galaxy $S_{N}$ dilution (and coined the term "superefficiently") based on a possible correlation of central galaxy $S_{N}$ with BM type; but as discussed above, our data show no such correlation.

One problem with the initial conditions scenario is the lack of any definite physical mechanism for deciding which protogalaxies will undergo GCs formation with enhanced efficiency. What royal jelly made M87 queen bee in the Virgo hive? Harris \& Pudritz (1994) constructed a detailed model for GC formation out of primordial pressure-confined, self-gravitating, magnetized supergiant molecular clouds. They conjecture that larger external pressures may have caused the primordial clouds in their model to fragment into more proto-GC cores per unit cloud mass, and that 
these higher pressures prevailed in the proto-halos of $\mathrm{cD}$ galaxies. Numerical modeling would appear necessary to prove this conjecture.

Initial conditions arguments are always difficult to rule out. On the other hand, it is clear that our data indicate that at least some of the reasons for resorting to initial conditions are no longer valid. In particular, it is not true that there are no correlations of $S_{N}$ with cluster properties. We find very good correlations between $S_{N}$ and cluster X-ray properties, as well as between $S_{N}$ and the velocity dispersion of the cluster galaxies, including the "asymptotic" dispersions. Thus, we do not believe that the central galaxies in our study formed their GCs "superefficiently" as a result of local initial conditions.

(2) Biasing. A similarly motivated, but fundamentally different model is that of "biased GC formation." This is the apparent heir of the early work by Peebles \& Dicke, the major addition being that of dark matter. Probably first to propose a model along these lines was Peebles (1984), and one of the most recent was West (1993). In the biasing scenario, GC formation depends only on the height of the local density fluctuations with respect to some universal threshold. When the small scale (parsec-sized) fluctuations are superposed on a larger scale (Mpc-sized), low amplitude fluctuation, such as might eventually evolve into a cluster of galaxies, the small scale fluctuations are more likely to broach this universal threshold; thus, they have a "bias" working in their favor. In such regions, the biasing may even result in the formation of GCs outside the sphere of influence of any particular galaxy. These intergalactic GCs then become associated with the whole of the cluster which will eventually form there. Thus, the biasing model is also primordial, but less "local" than the initial conditions model.

This model predicts that $S_{N}$ should correlate strongly with the present-day density of the local environment. We see this for the galaxies in our sample, primarily in the correlation of $S_{N}$ with cluster velocity dispersion. However, a possibly major problem with biasing is that, at least in some formulations (Peebles 1984), it requires GCs to have large dark matter halos. Such halos appear to be ruled out observationally (Moore 1996). Thus, although our data do not rule out this model, there are other 
reasons to be skeptical. Biasing has been proposed as a possible mechanism at work in the IGC model disucssed below; we will come back to it.

(3) Mergers. In this model, low $S_{N}$ gas-rich systems, such as spiral galaxies, merge to form elliptical galaxies, as suggested by Toomre (1977). In the process, GCs form out of shocked gas clouds, increasing the value of $S_{N}$ with every merger by an amount proportional to the available gas (Ashman \& Zepf 1992; Schweizer 1987). The physical mechanisms for creating GCs in such high speed colliding gas clouds were discussed in detail by Kumai et al. (1993a,b). The model assumes that mergers were common early in the history of a galaxy cluster, when the galaxies still had large amounts of gas. Repeated mergers of such systems in the cluster core formed more and more GCs around the central galaxy, resulting in a high $S_{N}$ system.

There is no doubt such merging does indeed take place, and that dense star clusters do form in the process (e.g., Holtzman 1992). Although van den Bergh (1995) has argued that these objects are not GCs because they do not follow the correct luminosity functions, we believe that further HST observations are needed in this regard. However, the question we must address is whether or not galaxy mergers are the driving force behind the variations in $S_{N}$ we see among central galaxies in clusters. As formulated (Ashman \& Zepf 1992; Zepf \& Ashman 1993), the merging model predicts a strong dependence of $S_{N}$ on galaxy luminosity, which we simply do not see in our sample, while we do see other correlations which are not immediately obvious in this picture. Thus, we reject mergers as the primary mechanism behind this particular phenomenon. (See Harris et al. [1995] for further arguments.)

(4) Stripping. The idea that the central galaxy in the cluster potential increases its $S_{N}$ through preferential tidal stripping of the GCs from other galaxies in the cluster goes back to Forte, Martinez, \& Muzzio (1982), and reached its fullest development in the paper by Muzzio (1987), who included a review of the numerical simulations. This model is out of fashion, and those who suggest it are promptly shouted down with a quick reference to van den Bergh (1984). However, although we fear to speak kindly of this model, we do not find the arguments against it to be fatal. In the discussion of Chapter 2 we noted that the numbers are not unreasonable, and that 
since the GC populations of elliptical galaxies tend to be more extended than the halo light (e.g. Harris 1991), the argument about the impossibility of the central galaxy increasing its $S_{N}$ in this process may not hold. We believe that this model was never given serious attention (cf. the remarks by McLaughlin et al. 1993).

The observed strong correlation of central galaxy $S_{N}$ with cluster velocity dispersion might occur in the stripping model, as the crossing time in high dispersion clusters is lower and each galaxy would have passed through the core more times (although higher velocities also make stripping less efficient). Moreover, the correlations with local galaxy density and location of the galaxy relative to the cluster center are both easily understandable in the context of this model. Stripping in the context of the IGC model is discussed below.

Muzzio (1987), perhaps unduly influenced by the stripping analogy, dubbed the stray GCs intergalactic "tramps." If this model is to be resurrected, we suggest the use of a more felicitous term such as "wayfarers" or "pilgrims." Such issues aside, there do appear to be some significant problems with this model. For instance, the increase of $S_{N}$ for the central galaxy was too slow in the simulations to explain the observations, and the stripped GCs should have low metallicities, in possible disagreement with observations (van den Bergh 1984), although see Elson \& Santiago (1996). Thus, we do not espouse this model as the clear winner.

(5) Cooling flows. It was pointed out by Fabian, Nulsen, \& Canizares (1984) that the amount of mass added to M87 in a gaseous state by its cooling flow was enough to account for the large number of GCs. As shown by Harris et al. (1995), no correlation exists between central galaxy $S_{N}$ and observed cooling flow rate; our data support this conclusion. For instance, while the A2199 cD has a large cooling flow and a fairly high $S_{N}$, the $S_{N}$ of NGC 4874 is still higher, although Coma does not appear to have a cooling flow. In addition A262-1 has a value of $S_{N}$ in the lower half of the observed range, though its cluster has a larger cooling flow, perhaps three times larger, than that found in Virgo. Thus, this model is clearly ruled out once again.

(6) Intracluster globular clusters (IGCs). The IGC model, developed by West et al. (1995) and mentioned previously when we examined correlations of $S_{N}$ with cluster 
properties ( $\$ 5.2 .3 .2)$, differs from all the rest in that it is phenomenological, rather than explanatory. It proposes that there are large populations of GCs which belong to the cluster as a whole and follow the overall cluster density in their distribution. The mechanisms suggested by West et al. for the origin of the IGCs are stripping, biasing, or perhaps some unknown process.

As stated previously, we have confirmed the prediction of the IGC model that the number of GCs in excess of $S_{N} \sim 4$ should correlate linearly with $T_{X} /\left(1+r^{2} / r_{c}^{2}\right)$. Moreover, West et al. predicted that the $S_{N}$ values of two of our sample galaxies, A569-1 and A779-1, would be relatively low, $S_{N} \sim 4$, and we confirm these predictions in both cases. Thus, we believe that the IGC model is on the right track in treating the GC populations of the central galaxies as more a property of the cluster itself than of the galaxy. However, in order to clump around the galaxy, the IGCs must have a velocity dispersion closer to the internal dispersion of the galaxy $\left(\sim 300 \mathrm{~km} \mathrm{~s}^{-1}\right)$ than of the cluster $\left(\sim 750 \mathrm{~km} \mathrm{~s}^{-1}\right)$. This is observed to be the case for M87 where the dispersion of the GCs is $\sim 400 \mathrm{~km} \mathrm{~s}^{-1}$ (Mould et al. 1990; Cohen 1996).

In addition, the origin of the IGCs remains a problem. The lack of dark halos around GCs might be an insurmountable problem for the biasing scheme. Although the alternative mechanism of stripping qualitatively explains our observations, it does not appear to be efficient enough to build up such a large IGC population, and there may be additional difficulties from metallicity considerations. Therefore, it is not clear that the GCs are truly intergalactic; what we mean by saying that they are "more a property of the cluster" is that their number is determined by cluster properties, not by the properties of the central galaxy.

We conclude that no clear winner has emerged from among the available models, though we have seen some strong contenders. In the following section we use as an additional guide the observationally known properties of the BCGs themselves. Along with the primary insight garnered through this discussion and our own observations, i.e., that the GCs are more a property of the cluster than the galaxy, we attempt to construct a coherent picture of the "BCG phenomenon" and the observed dependence of $S_{N}$ on cluster properties. 


\subsubsection{A Unified Model}

Discussions about why only certain central galaxies in clusters have "anomalously" high values of $S_{N}$ usually revolve around the presence of a $\mathrm{cD}$ envelope, which appears to be a helpful but not sufficient condition for having a high $S_{N}$. In an effort to uncover further clues in this regard, we extend the discussion to include another remarkable property of central cluster galaxies, namely their uniformity.

Sandage (1972) was the first to exploit the small dispersion in the metric luminosities $L_{m}$ of brightest cluster galaxies that was first noted by Humason, Mayall, \& Sandage (1956). He reported that the dispersion in absolute magnitude within a metric aperture of radius $\sim 20 h^{-1} \mathrm{kpc}$ was only $0.25 \mathrm{mag}$ in a data sample of 84 BCGs. Moreover, it showed no dependence on cluster richness over the entire richness range of his sample, spanning a factor of 100 . This apparent cosmic conspiracy, endowing every BCG with roughly the same luminosity independent of cluster size, has been called the "BCG phenomenon."

Schechter \& Peebles (1976) showed that a model in which BCGs are drawn at random from a universal galaxy luminosity function lessened the need for conspiracy theories, and they cautioned against the invocation of special mechanisms for forming BCGs. Tremaine \& Richstone (1977) found that the difference in the total magnitudes (i.e., including envelopes) of the first and second brightest cluster galaxies was too large on average to be accounted for by statistical sampling of any standard universal luminosity function. They concluded that the BCGs were therefore likely the products of a "special" dynamical process, but declined to speculate whether this process occurred during the formation of the galaxy or in its later evolution. (Note that the differences in the metric magnitudes of the first and second brightest galaxies are smaller than the differences in total magnitudes [Schneider et al. 1983b].)

Hoessel, Gunn, \& Thuan (1980) studied a larger, unbiased sample of 116 BCGs and concluded that the intrinsic dispersion in absolute magnitude within a metric radius of $10 h^{-1} \mathrm{kpc}$ was $\sim 0.35 \mathrm{mag}$, and did have a slight dependence on cluster richness, in the expected sense of central galaxies in richer clusters being brighter. Hoessel (1980) showed that this trend also correlated with the structure parameter 
$\alpha$, the slope of the luminosity profile at the metric radius. He defined the $L_{m}-\alpha$ distance indicator, which showed no residual dependence on richness. Hoessel found that correcting for BCG profile shape reduced the scatter in $L_{m}$ by $\sim 0.1$ mag.

Hoessel \& Schneider (1985) published CCD photometry of 175 BCGs, finding an intrinsic dispersion of $0.32 \mathrm{mag}$ in the uncorrected $L_{m}$. Oegerle \& Hoessel (1991) then moved away from the $L_{m}-\alpha$ indicator to study the "fundamental plane" $r_{e}-\sigma-$ $\left\langle\mu_{e}\right\rangle$ distance indicator for BCGs and its projection, the $r_{e}-\left\langle\mu_{e}\right\rangle$ indicator, where $r_{e}$ is the half light radius, $\sigma$ is the central stellar velocity dispersion, and $\left\langle\mu_{e}\right\rangle$ is the mean surface brightness interior to $r_{e}$. These authors concluded that the $r_{e}-\sigma-\left\langle\mu_{e}\right\rangle$ indicator gave distances to BCGs with an accuracy of $21 \%$, just as for lower luminosity ellipticals; however, although the $r_{e}-\left\langle\mu_{e}\right\rangle$ relation showed enormous scatter for other ellipticals, it yielded distances to BCGs accurate at the $25 \%$ level. Thus, the BCG phenomenon was nearly as good as using the whole fundamental plane, but much less expensive observationally. This finding approaches the heart of the conspiracy: for BCGs, the fundamental plane apparently collapses along its breadth.

Most recently, Lauer \& Postman (LP 1994; PL 1995) have reinvestigated the use of the $L_{m}-\alpha$ distance indicator for a sample of 119 BCGs. We have benefitted from their study, as it was the source from which our own sample was selected. PL find that the intrinsic dispersion in $L_{m}$ drops from $0.33 \mathrm{mag}$ to $0.24 \mathrm{mag}$ after the $\alpha$ correction has been applied, and residuals about the $L_{m}-\alpha$ relation show no dependence on BCG luminosity, color, or location, nor on cluster richness. Moreover, they report that $L_{m}$ is independent of cluster richness even before the $\alpha$ correction has been applied. They conclude that the small scatter in $L_{m}$ and $(B-R)$ color, coupled with the lack of any second-parameter effects, make BCGs "the most homogeneous distance indicators presently available for large-scale structure research."

The intent of this discussion has been to highlight the following points:

1. BCGs have a very small range in absolute metric luminosity $L_{m}$.

2. Any intrinsic scatter in $L_{m}$ shows little or no dependence on cluster properties.

3. In contrast, the globular cluster specific frequency $S_{N}$ of the BCGs in our sample has a range of more than a factor of 3 . 
4. $S_{N}$ is strongly correlated with cluster density as measured by velocity dispersion, $T_{X}, L_{X}$, and (less strongly) the local density of bright galaxies.

5. Taken together, these facts imply that BCGs with relatively high values $S_{N}$ do not have too many GCs for their luminosity; rather, they are underluminous with regard to their exalted positions in cores of dense clusters, whereas the number of GCs accurately reflects the dense environs.

To illustrate this view, Figure 5-39 plots our "region" absolute magnitudes (as in the lower panel of Figure 5-24) against cluster velocity dispersion. The term "region" is meant to distinguish from the $L_{m}$ metric magnitudes, which PL show to be independent of cluster properties. The regions of interest extend out to $32 h^{-1} \mathrm{kpc}$, and so are more likely to show a correlation, but as the figure illustrates, any trend has a large intrinsic scatter (the uncertainty in $M_{V}$ is of order $0.1 \mathrm{mag}$ ).

In marked contrast, Figure 5-40 shows the "GC excess" plotted against cluster dispersion. This is the same quantity discussed above in the context of the IGC model; it represents the number of GCs which elevate $S_{N}$ above a value of 3.5. The correlation is strong, and the only seriously discrepant points are the non-central galaxies (three of which are officially BCGs, but we discuss that further below). Thus, there is nothing anomalous about the numbers of GCs; they scale nicely with cluster density, indicating that they formed in proportion to the local mass density. There is no need to assume biasing.

What would be remarkable if it were not just a manifestation of the well-known "BCG phenomenon", is the lack of correlation between galaxy luminosity and environment. Thus, dividing the smoothly varying GC excesses in Figure 5-40 by the relatively invariant luminosities represented in Figure 5-39 (and adding 3.5) results in the good correlations of $S_{N}$ with cluster density, as seen for instance in Figure 5-27 and Figure 5-36.

If this view, that the "BCG phenomenon" creates the illusion of anomalous GC populations, is correct, then somehow the correlation of $S_{N}$ with galaxy extent, seen in Figure 5-25, must be explained. As mentioned previously, the $\alpha$ correction was originally introduced by Hoessel because both $\alpha$ and $L_{m}$ showed similar small cor- 


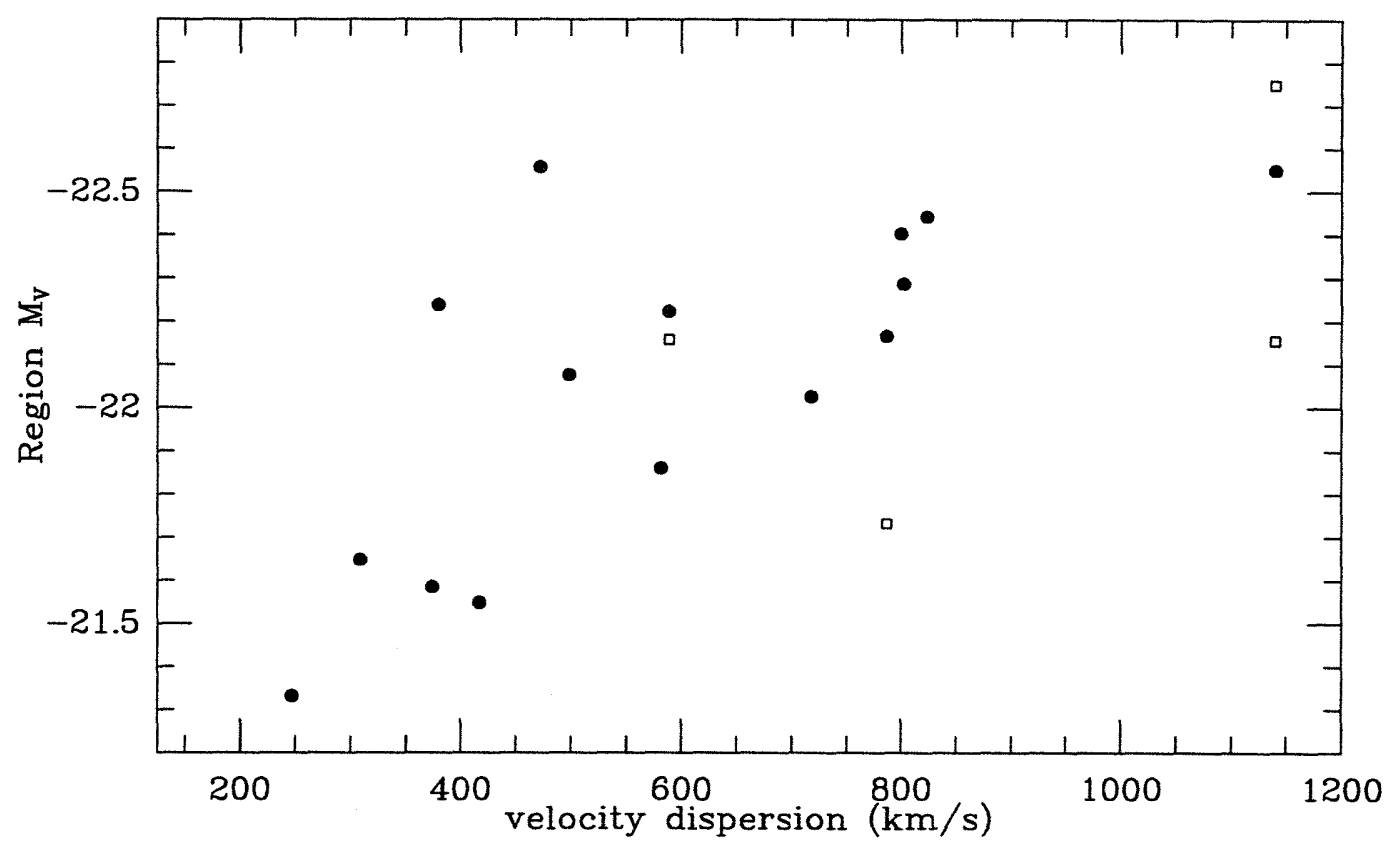

Figure 5-39: Total absolute $V$ magnitude of each region galaxy in which we have measured our metric $S_{N}$ values (see Figure 5-24) is plotted against cluster velocity dispersion. Non-central galaxies are shown as open squares.

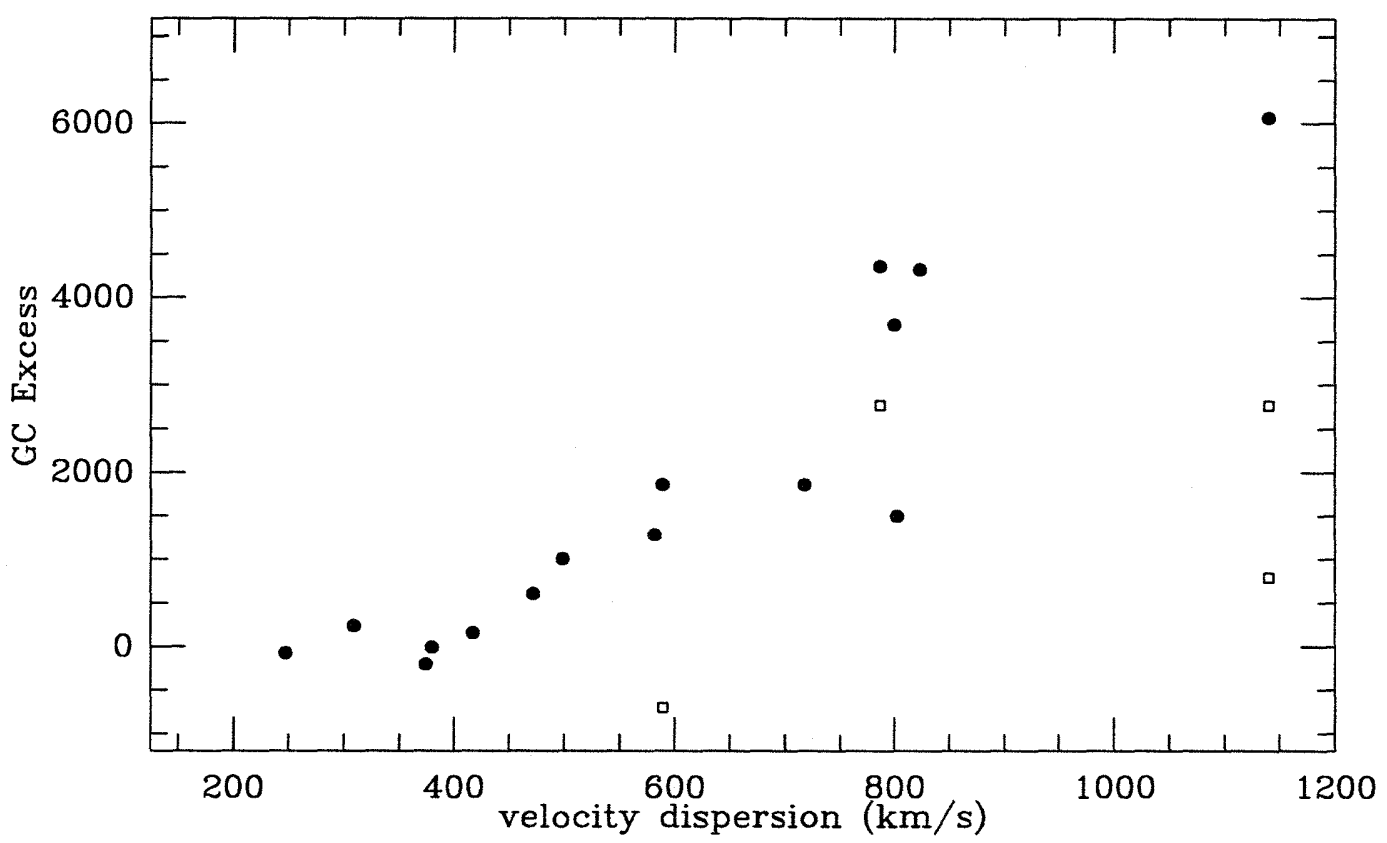

Figure 5-40: The excess GC number above $S_{N}=3.5$ is plotted against cluster velocity dispersion. Non-central galaxies are shown as open squares. 


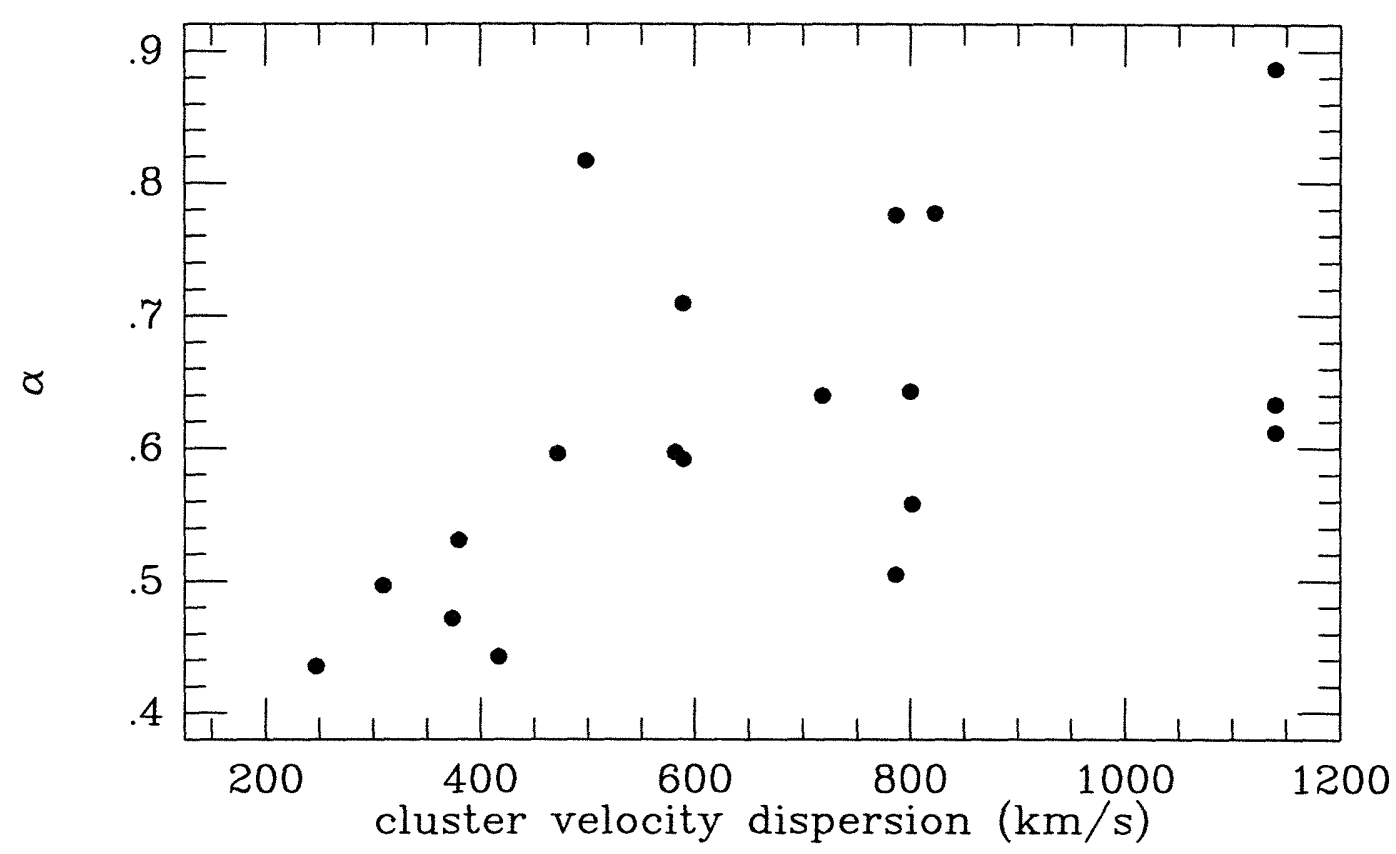

Figure 5-41: The correlation between BCG profile structure parameter $\alpha$ and cluster velocity dispersion.

relations with cluster richness. Thus, it was possible to construct a combination of the two which had smaller scatter and no dependence on cluster properties. In Figure 5-41, we show that $\alpha$ does indeed correlate with cluster velocity dispersion. (The biggest "outlier" in the plot, with $\alpha \sim 0.8$ but a low cluster dispersion is A262-1, which has a central dust lane.) Thus, given the small scatter in the relationship between $S_{N}$ and velocity dispersion seen in Figure 5-27, it is not at all surprising that $S_{N}$ would correlate with $\alpha$ as well. However, since the significance of the correlation between $S_{N}$ and dispersion is very nearly 1.0, and the $S_{N^{-}} \alpha$ and $\alpha$-dispersion correlations are both formally significant at the $\sim 0.998$ level, we cannot determine which of the latter two is more "fundamental." A remaining mystery is that PL find no correlation of "raw" metric luminosity with cluster properties, then apply a correction factor based on $\alpha$, which does correlate with at least cluster dispersion, to decrease the scatter in the $L_{m}$ indicator by nearly $25 \%$ but without re-introducing any dependence on cluster properties. We leave that for the reader to ponder.

In light of all the correlations between $S_{N}$, cluster density, galaxy position within the cluster center, and $\alpha$, we offer one comment on the process of BCG selection. If 
it is true that the large variations in $S_{N}$ are due to the relative constancy of galaxy luminosity known as the "BCG phenomenon", then it seems clear that the galaxy chosen for BCG distance surveys, which rely on this "phenomenon", should be the one with the higher value of $S_{N}$, since this will be the one which has experienced the luminosity limitation, thereby becoming partner to the conspiracy. This will also be the more central galaxy in the cluster and the one with the larger value of $\alpha$. Thus for their respective clusters, the "correct" galaxies would be A539-2, A1656-2, and A2197-2 (this last one was in fact selected by Hoessel et al. 1980).

Finally, we close with some discussion as to why the total GC number fails to be limited in the same fashion as central galaxy luminosity, yielding the observed correlation of $S_{N}$ with cluster density. The most obvious explanation is simply that the GCs of these galaxies, like those of the Milky Way, are ancient, as many lines of evidence from spatial, color, and dynamical distributions suggest (e.g. Harris 1991; van den Bergh 1995). Thus, the GCs all formed before whatever limiting mechanism occurred to halt further luminosity growth of the central galaxy. We suggest that the limiting mechanism was rich cluster formation itself. A possible scenario might run as follows.

In hierarchical models of structure formation, objects the size of GCs form early on due to their short collapse times (e.g. Peebles \& Dicke 1968; Tegmark et al. 1996), with perhaps $0.1 \%$ of the proto-galactic gas ending up as stars inside GCs (cf. Larson 1990). Large galaxies, on the other hand, have more chaotic formation histories, probably spanning several billion years, as envisioned by Searle \& Zinn (1978). During this period, the densest modern day clusters would already commence forming.

In his investigation of cluster evolution, Merritt (1984) concluded that the essential properties of both galaxy clusters and their central dominant galaxies are determined by dynamical processes which occurred prior to, or during, the epoch cluster formation. Thus, we expect that a giant galaxy destined for central location in a cluster would have originated near the center of the cluster-sized overdensity, with an initial store of gas which depended on the size of that overdensity, and would have formed a proportionate number of GCs. The tidal stresses of rich cluster collapse might then 
rob the forming galaxy of its gas supply in a manner similar to the "galaxy harassment" model of Moore et al. (1996), but faster, due to the higher densities of the early cluster core. (Merritt points out that during the initial collapse the core density may briefly exceed its later virialized density by a factor of several.)

Since the onset of cluster formation depends on the size of the overdensity, higher density clusters would form before lower density ones, and their initially bigger central protogalaxies would be robbed of their gas supply sooner. The resulting "coincidence" of constant BCG luminosity would then have as a collateral result the strong correlation of $S_{N}$ with cluster velocity dispersion and other measures of cluster density. Delayed luminosity growth of the central galaxy in a dense cluster through the process of galactic cannibalism is apparently too slow (Blakeslee \& Tonry 1992) to cause any significant later decrease in $S_{N}$. In addition, the greater "harassment" experienced in the formation of denser clusters might cause the galaxy to bloat and distend, yielding the observed correlations with galaxy profile.

This scenario also explains why no galaxies with very high $S_{N}$ values are found outside clusters. We note that similar arguments have been made, for different reasons, in favor of the growth of $\mathrm{cD}$ envelopes through dynamical processes that predated cluster formation (Merritt 1984, 1988; Schombert 1988); thus, the frequent, but not absolute, association of $\mathrm{cD}$ envelopes and high values of $S_{N}$ appears understandable from this perspective.

Of course, for a model to have worth, it must have some predictive power. The most obvious prediction of this "BCG saturation" model of $S_{N}$ variation is that, if star formation is halted only because of gas loss, then central galaxies with larger $S_{N}$ should also have more dark matter; thus $S_{N}$ should correlate with mass-to-light ratio $M / L$. This is a difficult quantity to measure well, as information on the internal stellar velocity dispersion profile is needed, not just a central dispersion. However, some supporting evidence may come from a comparison of M87 with NGC 4472, the brightest galaxy in Virgo. Although these galaxies have similar luminosities, the central galaxy M87 has about 3 times as many GCs. It is interesting that Mould et al. (1990) find that $M / L$ measured within a radius of $8^{\prime}\left(\sim 30 h^{-1} \mathrm{kpc}\right)$ is roughly 5 times 
larger for M87 than for NGC 4472, but a true test must come from a comparison of $M / L$ for central galaxies with known $S_{N}$ in different clusters.

A less obvious prediction of this scenario is that since the central galaxies with higher $S_{N}$ would have processed less of their initial gas supply, they would be expected to have lower metallicities than central galaxies with lower $S_{N}$ values. PL find that the $(B-R)$ colors of these galaxies are remarkably uniform, and we have found no significant trends of $S_{N}$ with this color that might indicate the expected metallicity dependence. However, we defer modification of this model pending both better tests against actual metallicity indices and further elaboration of the model, which has been sketched only qualitatively here.

\subsection{References}

Aaronson, M., Bothun, G., Mould, J., Huchra, J., Schommer, R. A., \& Cornell, M. E. 1986, ApJ, 302, 536

Aaronson, M. et al. 1989, ApJ, 338, 654

Abell, G. O., Corwin H. G. Jr. \& Olowin, R. P. 1989, ApJS, 70, 1

Abraham, R. G. \& van den Bergh, S. 1995, ApJ, 438, 218

Ajhar, E. A., Blakeslee, J. P., \& Tonry, J. L. 1994, AJ, 108, 2087

Ajhar, E. A., Tonry, J. L., Dressler, A., \& Blakeslee, J. P. 1997, in preparation

Ashman, K. M. \& Zepf, S. E. 1992, ApJ, 384, 50

Bahcall, N. A. \& Oh, S. P. 1996, ApJ, 462, L49

Bahcall, N. A. 1981, ApJ, 247, 787

Baum, W. A. et al. 1995, AJ, 110, 2537

Beers, T. C., Forman, W., Huchra, J. P., Jones, C., \& Gebhardt, K. 1991, AJ, 102, 1581

Beers, T. C. \& Tonry, J. L. 1986, ApJ, 300, 557

Blakeslee, J. P. \& Tonry, J. L. 1992, AJ, 103, 1457

Blakeslee, J. P. \& Tonry, J. L. 1995, ApJ, 442, 579

Bridges, T. J., Carter, D., Harris, W. E. \& Pritchet, C. J. 1996, MNRAS, submitted 
(Astro-ph/9604159)

Bridges, T. J., Hanes, D. A., \& Harris, W. E. 1991, ApJ, 101, 469

Butterworth, S. T. \& Harris, W. E. 1992, AJ, 103, 1828

Cohen, J. G. 1996, personal communication

de Vaucouleurs, G., de Vaucouleurs, M., \& Corwin, H. G. 1976, Second Reference

Catalog of Bright Galaxies (Austin: University of Texas Press), (RC2)

de Vaucouleurs, G., de Vaucouleurs, A., Corwin, H. G., Jr., Buta, R. J., Paturel,

G., \& Fouqué, P. 1991, Third Reference Catalog of Bright Galaxies (New York:

Springer), (RC3)

Djorgovski, S. \& Santiago, B. X. 1992, ApJ, 391, L85

Edge, A. C. \& Stewart, G. C. 1991, MNRAS, 252, 428

Elson, R. A. W. \& Santiago, B. X. 1996, MNRAS, 280, 971

Fabian, A. C., Nulsen, P. E. J., \& Canizares, C. R. 1984, Nature, 310, 733

Fadda, D., Girardi, M., Giuricin, G., Mardirossian, F., Mezzetti, M. \& Biviano, A. 1996, ApJ, submitted (Asto-ph/9606098)

Forbes, D. A. 1996, AJ, 112, 954

Forbes, D. A., Brodie, J., \& Huchra, J. P. 1996b, AJ, submitted

Forbes, D. A., Franx, M., Illingworth, G. D., \& Carollo, C. M. 1996a, ApJ, 467, 126

Forte, J. C., Martinez, R. E., \& Muzzio, J. C. 1982, AJ, 87, 1465

Girardi, M., Biviano, A., Giuricin, G., Mardirossian, F., \& Mezzetti, M. 1993, ApJ, 404,38

Girardi, M., Biviano, A., Giuricin, G., Mardirossian, F., \& Mezzetti, M. 1995, ApJ, 438,527

Girardi, M., Fadda, D., Giuricin, G., Mardirossian, F., Mezzetti, \& M. Biviano, A. 1996, ApJ, 457, 61

Graham, A., Lauer, T. R., Colless, M., \& Postman, M. 1996, ApJ, 465, 534

Harris, W. E. 1981, ApJ, 251, 497

Harris, W. E. 1986, ApJ, 91, 822

Harris, W. E. 1991, ARA\&A, 29, 543

Harris, W. E., Allwright, J. W. B., Pritchet, C. J., \& van den Bergh, S. 1991, 76, 115 
Harris, W. E., Pritchet, C. J., \& McClure, R. D. 1995, ApJ, 441, 120

Harris, W. E. \& Pudritz, R. E. 1994, ApJ, 429, 177

Hoessel, J. G. 1980, ApJ, 241, 493

Hoessel, J. G., Gunn, J. E., \& Thuan T. X. 1980, ApJ, 241, 486

Hoessel, J. G. \& Schneider, D. P. 1985, AJ, 90, 1648 (HS)

Holtzman, J. et al. 1992, AJ, 103, 691

Huchra, J. P., Henry, J. P., Postman, M., \& Geller, M. J. 1990, ApJ, 365, 66

Humason, M. L., Mayall, N. U. \& Sandage, A. R. 1956, AJ, 61, 97

Jones, C. \& Forman, W. 1996, in preparation, almost ready for ApJ submission

Jones, C. 1996, private communication

Kissler-Patig, M., Kohle, S., Hilker, M., Richtler, T., Infante, L., Quintana, H. 1996, $\mathrm{A} \& \mathrm{~A}$, in press

Kumai, Y., Basu, B., \& Fujimoto, M. 1993a, ApJ, 404, 144

Kumai, Y., Hashi, Y., \& Fujimoto, M. 1993b, ApJ, 416, 576

Larson, R. B. 1990, PASP, 102, 709

Lauer, T. R. \& Postman, M. 1994, ApJ, 425, 418 (LP)

Lin, H., Kirshner, R. P., Shectman, S. A., Landy, S. D., Oemler, A., Tucker, D. L., \& Schechter, P. L., 1996, ApJ, 464, 60

Lubin, L. M. \& Bahcall, N. A. 1993, ApJ, 415, L17

Lucey, J. R., Gray, P. M., Carter, D. \& Terlevich, R. J. 1991, MNRAS, 248, 804

McLaughlin, D. E., Harris, W. E., \& Hanes, D. A. 1993, 409, L45

McLaughlin, D. E., Harris, W. E., \& Hanes, D. A. 1994, 422, 486

Merritt, D. 1984, ApJ, 276, 26

Merritt, D. 1988, in The Minnesota Lectures on Clusters of Galaxies and Large-Scale

Structure, ed. J. M. Dickey (ASP Conf. Ser., 5), 175

Moore, B. 1996, ApJ, 461, L13

Moore, B., Katz, N., Lake, G., Dressler, A., \& Oemler, A. Jr. 1996, Nature, 379, 613

Mould, J. R., Oke, J. B., de Zeeuw, P. T., \& Nemec, J. M. 1990, AJ, 99, 1823

Mushotzsky, R. 1984, Phys. Scripta, T7, 157

Muzzio, J. C. 1987, PASP, 99, 245 
Nichol, R. C., Collins, C. A., Guzzo, L., \& Lumsden, S. L. 1992, MNRAS, 255, 24p

Oegerle, W. R. \& Hoessel, J. G. 1991, ApJ, 375, 15

Peebles, P. J. E. 1984, ApJ, 277, 470

Peebles, P. J. E. \& Dicke, R. H. 1968, ApJ, 154, 891

Postman, M., Huchra, J. P. \& Geller, M. J. 1992, ApJ, 384, 404

Postman, M. \& Lauer, T. R. 1995, ApJ, 440, 28 (PL)

Pritchet, C. J. \& Harris, W. E. 1990, ApJ, 355, 410

Sandage, A. 1972, ApJ, 178, 1

Schechter, P. L. 1976, ApJ, 203, 297

Schechter, P. L. \& Peebles, P. J. E. 1976, ApJ, 209, 670

Schneider, D. P., Gunn, J. E., \& Hoessel, J. G. 1983a, ApJ, 264, 337

Schneider, D. P., Gunn, J. E., \& Hoessel, J. G. 1983b, ApJ, 268, 476

Schweizer, F. 1987, in Nearly Normal Galaxies, ed. S. M. Faber (New York: Springer), 18

Schombert, J. M. \& West, M. J. 1990, ApJ, 363, 331

Scodeggio, M., Solanes, J. M., Giovanelli, R., \& Haynes, M. P. 1995, ApJ, 444, 41

Searle, L. \& Zinn, R. 1978 ApJ, 225, 357

Secker, J. 1992, AJ, 104, 1472

Secker, J. \& Harris, W. E. 1993, AJ, 105, 1358

Silbermann, N.A. et al. 1996, poster presented May 1996 at the STScI Symposium on the Extragalactic Distance Scale

Struble, M. F. \& Rood, H. J. 1987, ApJS, 63, 555

Struble, M. F. \& Rood, H. J. 1991, ApJS, 77, 363

Tegmark, M., Silk, J., Rees, M. J., Blanchard, A., Abel, T., \& Palla, F. 1996, ApJ, in press

Tonry, J. L., Ajhar, E. A., \& Luppino, G. A. 1990, AJ, 100, 1416

Tonry, J. L., Blakeslee, J. P., Ajhar, E. A., \& Dressler, A. 1997, ApJ, 475, in press

Toomre, A., 1977, in The Evolution of Galaxies and Stellar Populations, eds. B. M.

Tinsley and R. B. Larson (New Haven: Yale Univ. Observatory), 401

Tremaine, S. D. \& Richstone, D. O. 1977, ApJ, 212, 311

Tyson, J. A. 1988, AJ, 96, 1 
van den Bergh, S. 1984, PASP, 96, 329

van den Bergh, S. 1992, PASP, 104, 861

van den Bergh, S. 1995, ApJ, 450, 27

van den Bergh, S. 1996, PASP, 108, in press

West, M. J. 1993, MNRAS, 265, 755

West, M. J., Côté, P., Jones, C., Forman, W., \& Marzke, R. O. 1995, ApJ, 453, L77

Whitmore, B. C., Sparks, W. B., Lucas, R. A., Macchetto, F. D., \& Biretta, J. A. 1995, ApJ, 454, L73

Worthey, G. 1996, private communication

Yahil, A. \& Vidal, N. 1977, ApJ, 214, 347

Zabludoff, A. I., Huchra, J. P., \& Geller, M. J. 1990, ApJS, 74, 1

Zabludoff, A. I., Geller, M. J., Huchra, J. P., \& Ramella, M. 1993, AJ, 106, 1273

Zepf, S. E. \& Ashman, K. M. 1993, MNRAS, 264, 611

Zucca, E., Zomorani, G. Scaramella, R., \& Vettolani, G. 1993, ApJ, 407, 470 


\section{Chapter 6}

\section{Summary and Conclusions}

In this thesis, we have studied the largest sample to date of GC populations around central galaxies in Abell clusters, 7 times larger than any previous single study. The improved analysis methods were the primary factor in allowing us to do this with a relatively small telescope for the standards of today. In addition, we were graced with a plentiful amount of observing time and favorable seeing conditions.

The mathematical basis for our technique, which uses both direct counts and surface brightness variances, was developed in Chapter 2 and applied to $I$-band images of NGC 4889 (A1656-1) and NGC 4874 (A1656-2) in Coma. The latter galaxy, cloaked in a cD envelope, was found to have a significantly higher specific frequency $S_{N}$, twice as high as the former galaxy. However, with the improved photometry discussed in Chapter 5, this ratio was revised downward to a factor of 1.5.

It was noted that our technique, as well as previous studies which used only direct counts, relied to some degree on the predictability of the GCLF turnover magnitude $m^{0}$. That is, the frequently made, but poorly tested, assumption of a universal GCLF was an important input. For the intercomparison of the two Coma galaxies, this fact was less important because they were at the same distance and shared the same environment. Before undertaking a larger study of a more diverse sample, a reinvestigation of the constancy of $M^{0}$ was warranted.

In Chapter 3, the universal GCLF hypothesis was weighed in the balance and found it wanting, at least in some regards. For instance, there is strong evidence 
that it differs as a function of environmental properties (including metallicity, but perhaps also dynamical factors) between rich clusters and poors groups. As the $m^{0}$ variation appeared dictated by environment, we continued onward to study the GCs in rich clusters, gambling that $m^{0}$ would not show strong variations from cluster core to cluster core.

In Chapter 4, we presented the Abell cluster data sample, all observations being in the $R$-band except for that of A2199. Image reductions were described in detail, including galaxy photometry and modeling, point source photometry and completeness tests, and power spectrum measurements. A personally favorite feature of that chapter was the sequence of isophotal contour plots for all the galaxies in the sample, including the Coma galaxies from Chapter 2. For several clusters, the second ranked galaxy (SRG) was included because it was comparable to the BCG in luminosity. In fact, in all cases, it was also more centrally located, as judged by both position relative to the $\mathrm{X}$-ray center and the local galaxy density. In addition, a third Coma galaxy, NGC 4839 (A1656-3), was included by virtue of its cD envelope.

In Chapter 5, we presented the analysis of our sample of Abell cluster galaxies. The radial point source distributions clearly indicated that a GC population had been detected around each galaxy. We then discussed background estimation and simultaneous constraints on $S_{N}$ and the GCLF width $\sigma$. Measurements of $\sigma$ for all the galaxies were presented, and we found a slight bias in those which were poorly constrained. However, the more tightly constrained values showed very little intrinsic dispersion, and agreed closely with the values found by deep imaging of nearby ellipticals, particularly M87, which was used as the " $m^{0}$ calibrator." This result confirmed our working assumption of a universal GCLF for central galaxies in the cores of rich clusters.

To limit biased comparisons of $S_{N}$ among sample galaxies, we assumed a single value of $\sigma$, consistent with our data, and calculated metric values of $S_{N}$, defined within $32 h^{-1}$ kpc. Our results for $S_{N}$ followed a continuous distribution; they were not separated into "normal" and "high", or "anomalous", classes. This was the first time in which a continuum of $S_{N}$ values was clearly shown to exist. 
Interestingly, and contrary to all past studies, we found strong correlations of $S_{N}$ with properties of the galaxy clusters, including central and asymptotic velocity dispersions, X-ray luminosity and temperature, and local galaxy density. For clusters with multiple representatives in our sample, the one with the higher $S_{N}$ value was always the one which was also more extended and closer to the cluster X-ray center.

We did not find a strong correlation with cluster richness expressed in Abell counts, but that was not surprising, given their fairly qualitative and uncertain nature. We also found no evidence of a previously proposed correlation of central galaxy $S_{N}$ with Bautz-Morgan class. No correlation was evident in the plot of $S_{N}$ with Rood-Sastry type either, although imaginative speculations were not ruled out.

A detailed discussion of proposed models for the variation of $S_{N}$ in central cluster galaxies was provided. Our data confirmed several predictions of the "intracluster globular cluster" (IGC) model. However, the lack of a viable mechanism for producing the IGCs was judged problematic. The stripping model appeared most consistent with our data, but there are other arguments which render it dubious, the primary one being the long time scale required to add GCs to the central galaxy.

We concluded the thesis by offering a synthesis, a unified view of the observed $S_{N}$ variations and the "standard candle" aspect of BCGs, at least following a correction for profile extent. In this view, GCs form early and in proportion to the local mass density, thus the observed excellent correlations of their number with cluster velocity dispersion and X-ray temperature. There is no need to invoke dark matter biasing. On the other hand, the luminosity of the central galaxy saturates, perhaps due to the process of cluster formation itself. The collapse and subsequent virialization of a cluster from many smaller, irregular groups of galaxies would certainly have stirred up the gas in a large, centrally located galaxy, halting subsequent star formation, adding significantly to the hot intracluster gas, and perhaps leaving as its signature the characteristic shallow profiles of these galaxies.

Although the scenario remains uncertain, it is clear that, for whatever reason, GC number scales with cluster properties (and should therefore itself be considered a cluster property), while BCG luminosity does not. We have proposed that the same 
situation gives rise to both the high values of $S_{N}$ measured for some galaxies, and the suitability of BCGs as "standard candles." The correlation we observed between $S_{N}$ and the profile structure parameter $\alpha$ is then understandable as a consequence of the fact that both of these quantities depend on cluster density. In light of these results, we suggested that the proper galaxy to use in BCG distance studies based on the $L_{m}-\alpha$ indicator or its variants is the bright extended galaxy closest to the cluster center, whether or not it happens to have the largest luminosity within $\sim 10 h^{-1} \mathrm{kpc}$. Our data and this interpretation predict that this galaxy will also be the one with the highest $S_{N}$.

These exciting results, and the interpretation we have offered, warrant further scrutiny, particularly in the form of further observational data. We have acquired comparable, but generally deeper, images of central galaxies in $\sim 10$ southern ACO rich clusters with the Cerro Tololo $4 \mathrm{~m}$ telescope. These could not be included in this thesis (time scale problems), but will serve to either support these observations and conclusions, or to make matters yet more interesting. 


\section{Appendix A}

\section{Probability Contours for $S_{N}$ and $\sigma$}

In this appendix, we display the probably contours on $S_{N}$ and GCLF $\sigma$ in each of 4 regions in all the sample galaxies, except for A1177-1, A2197-1, and A2199-1, in all of which the innermost regions were unusable. Relevant discussion of these figures can be found in Chapter 5. 


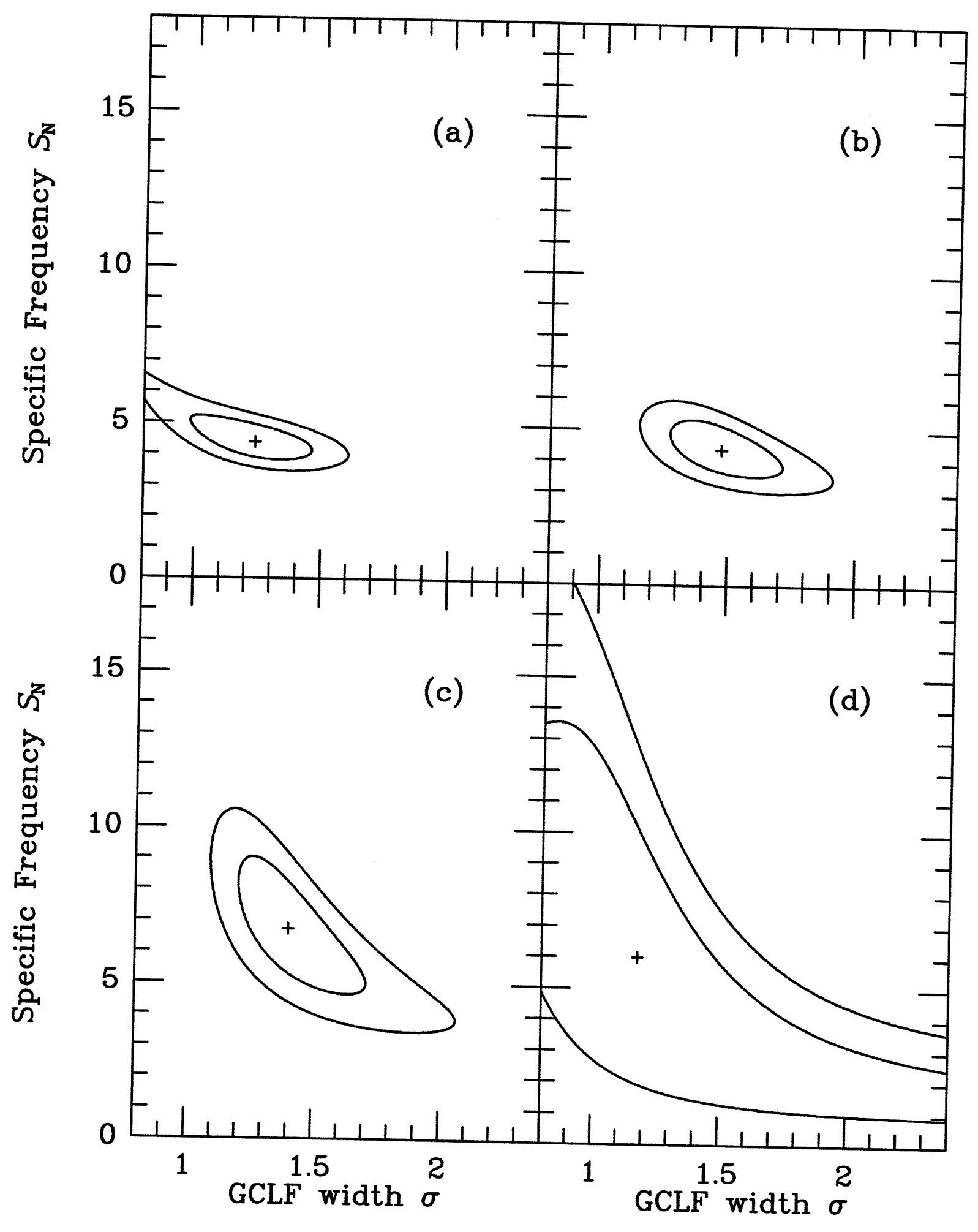

Figure A-1: A262-1 


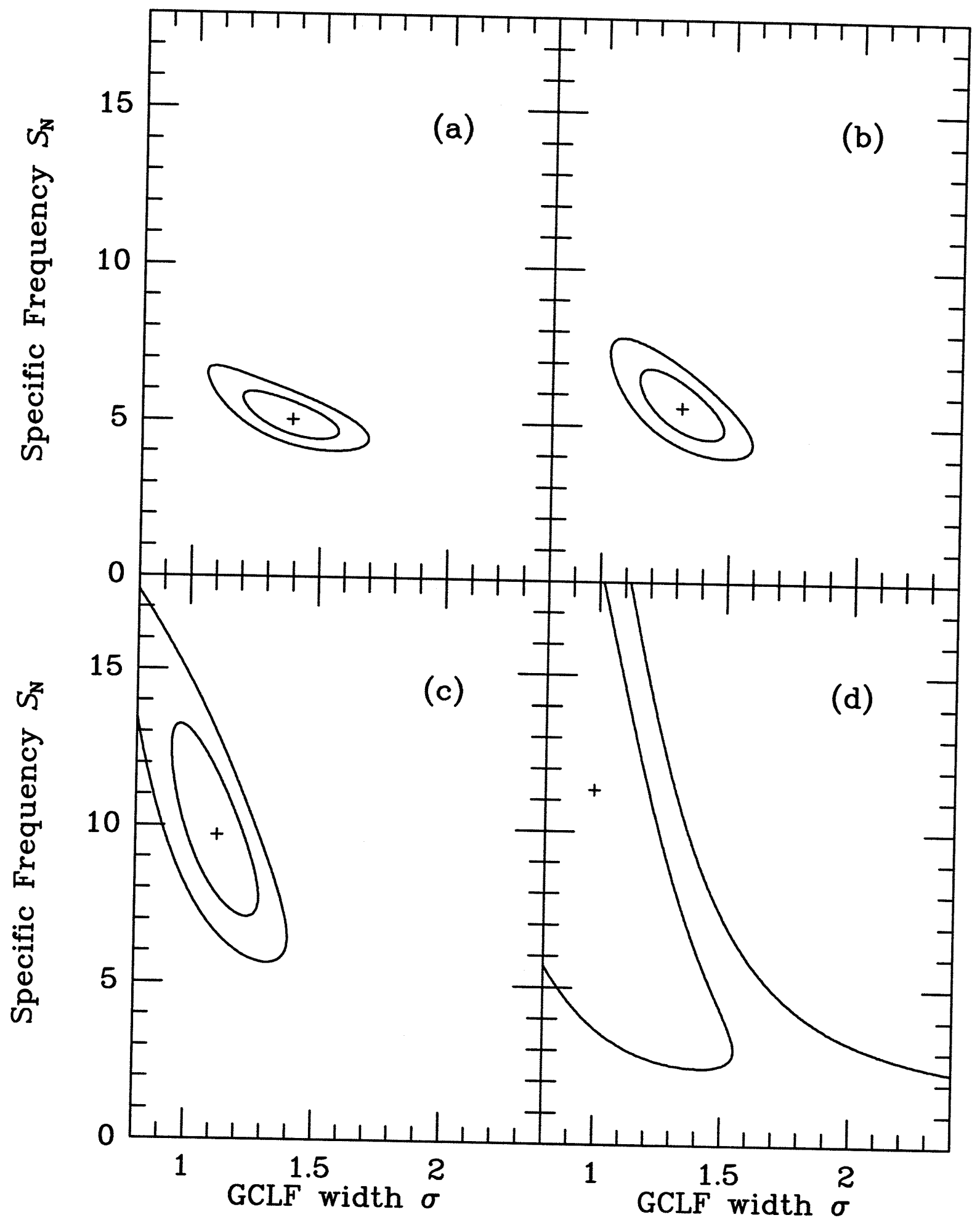

Figure A-2: A347-1 (1194). 


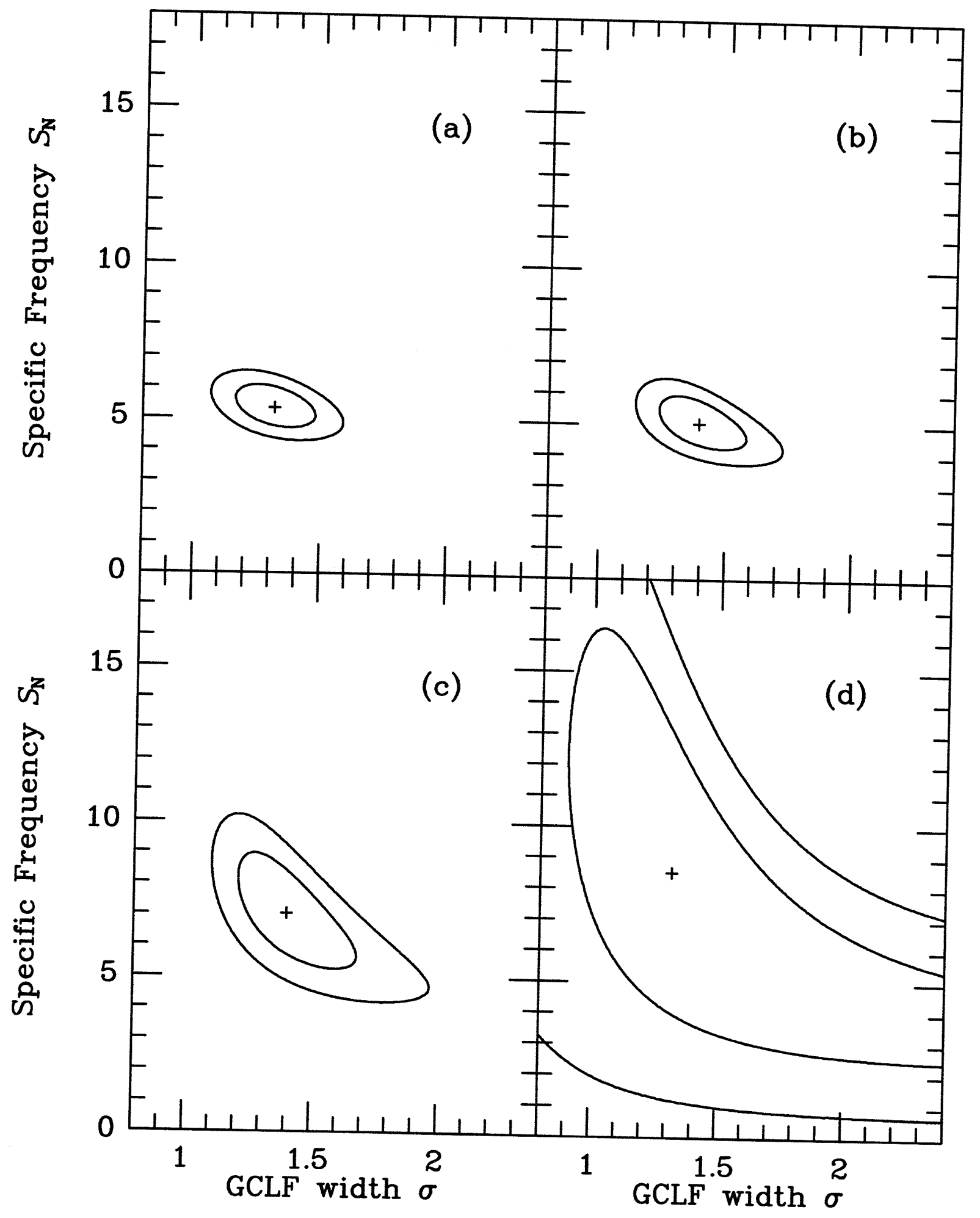

Figure A-3: A347-1 (0995). 


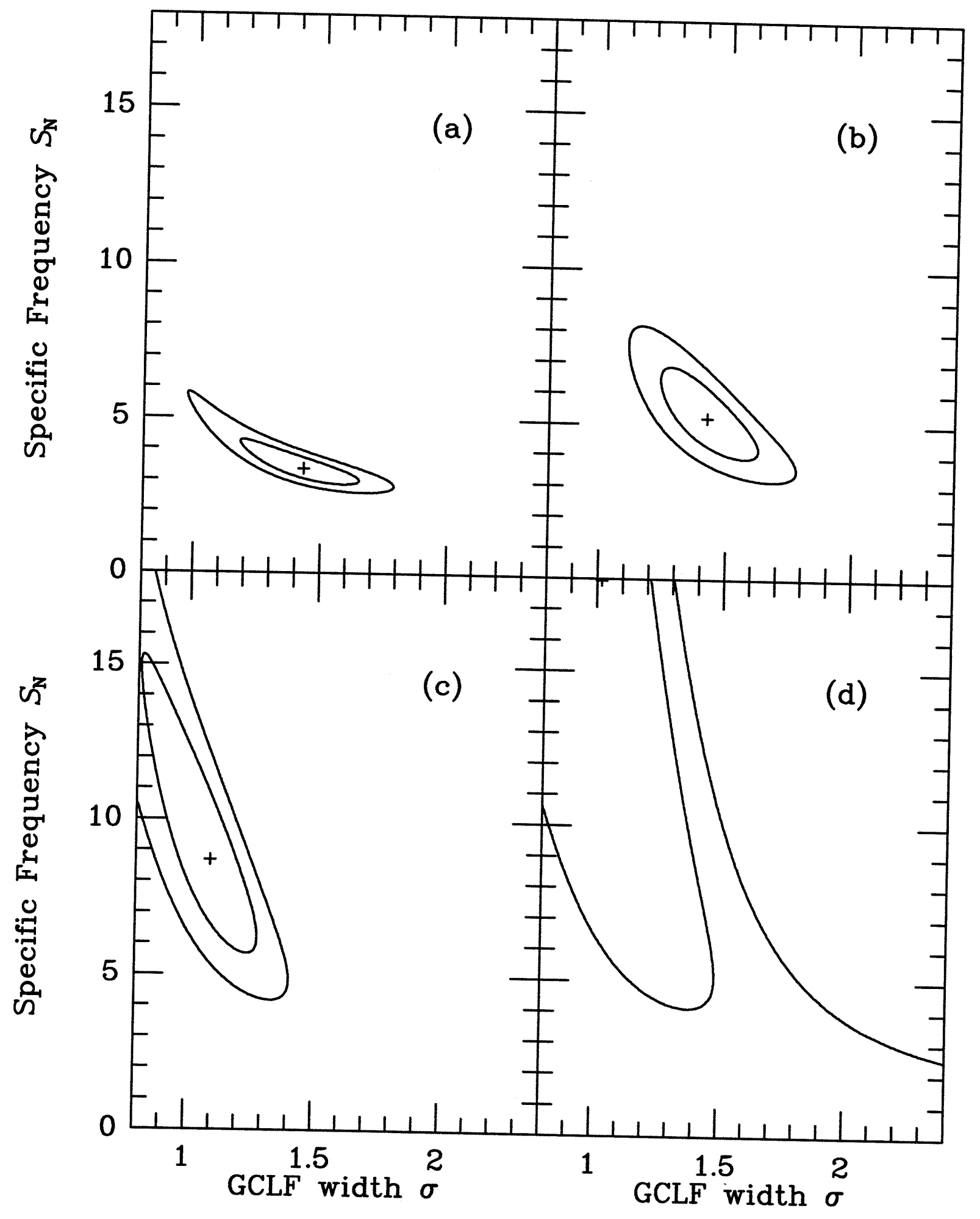

Figure A-4: A397-1. 


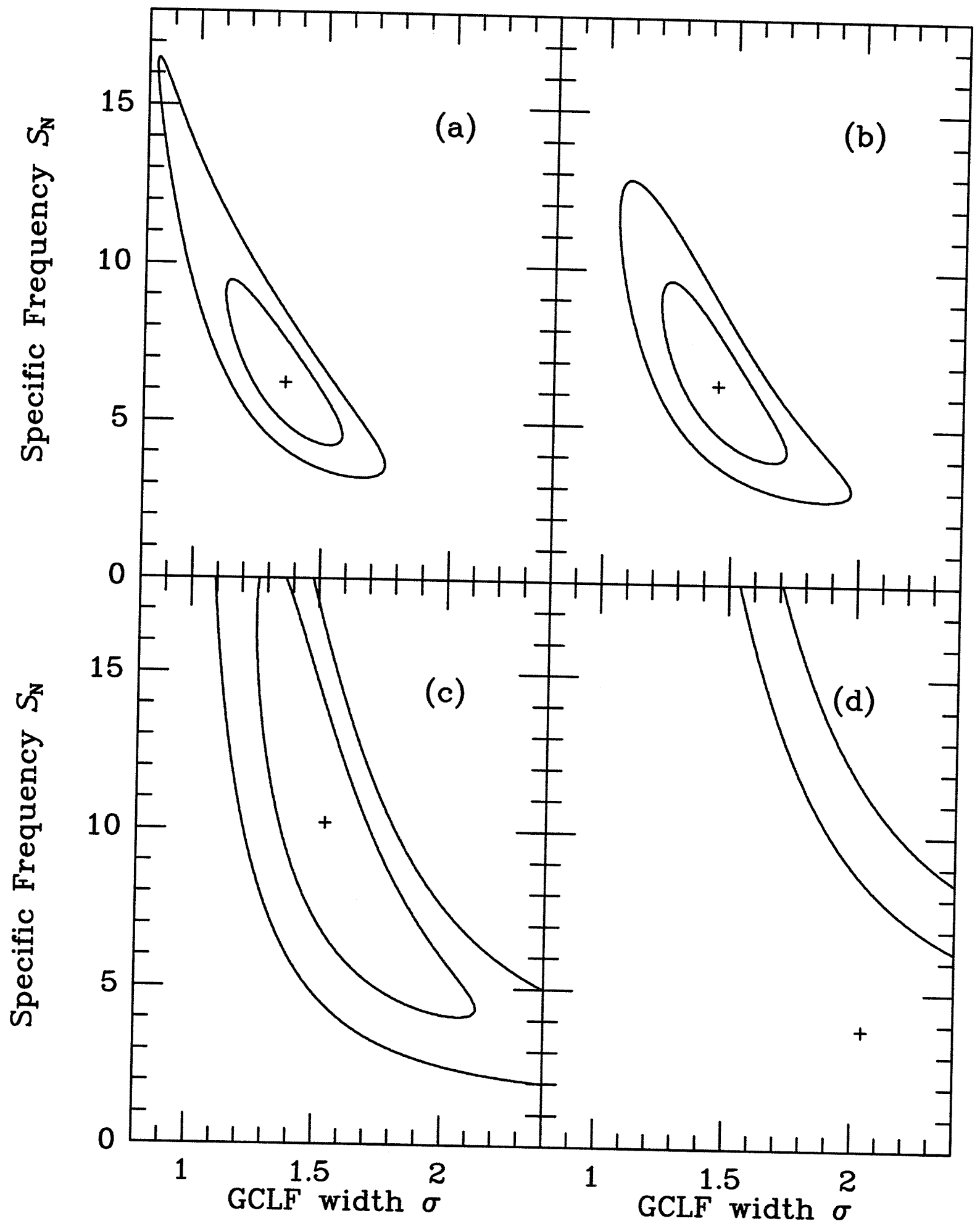

Figure A-5: A539-1. 


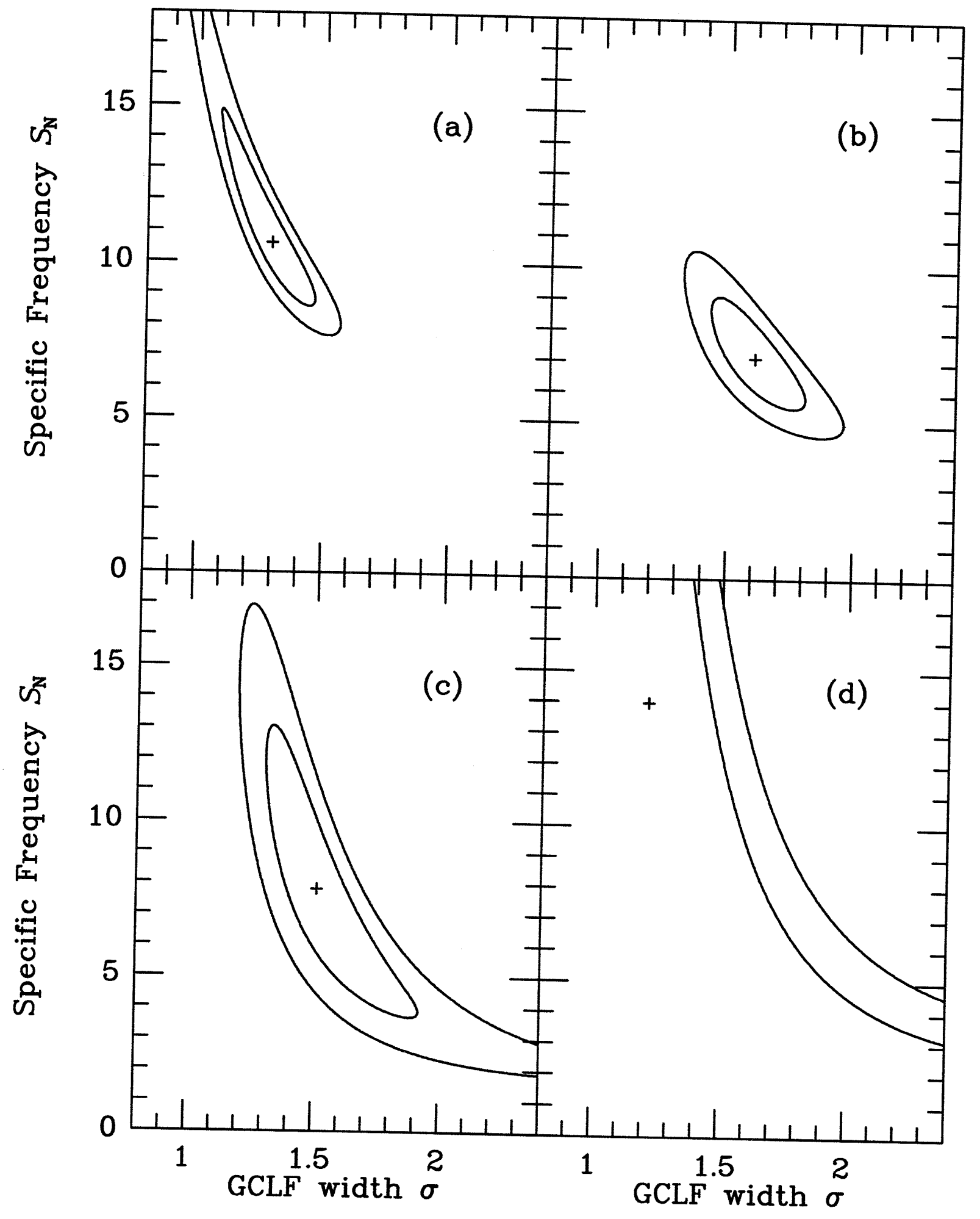

Figure A-6: A539-2. 


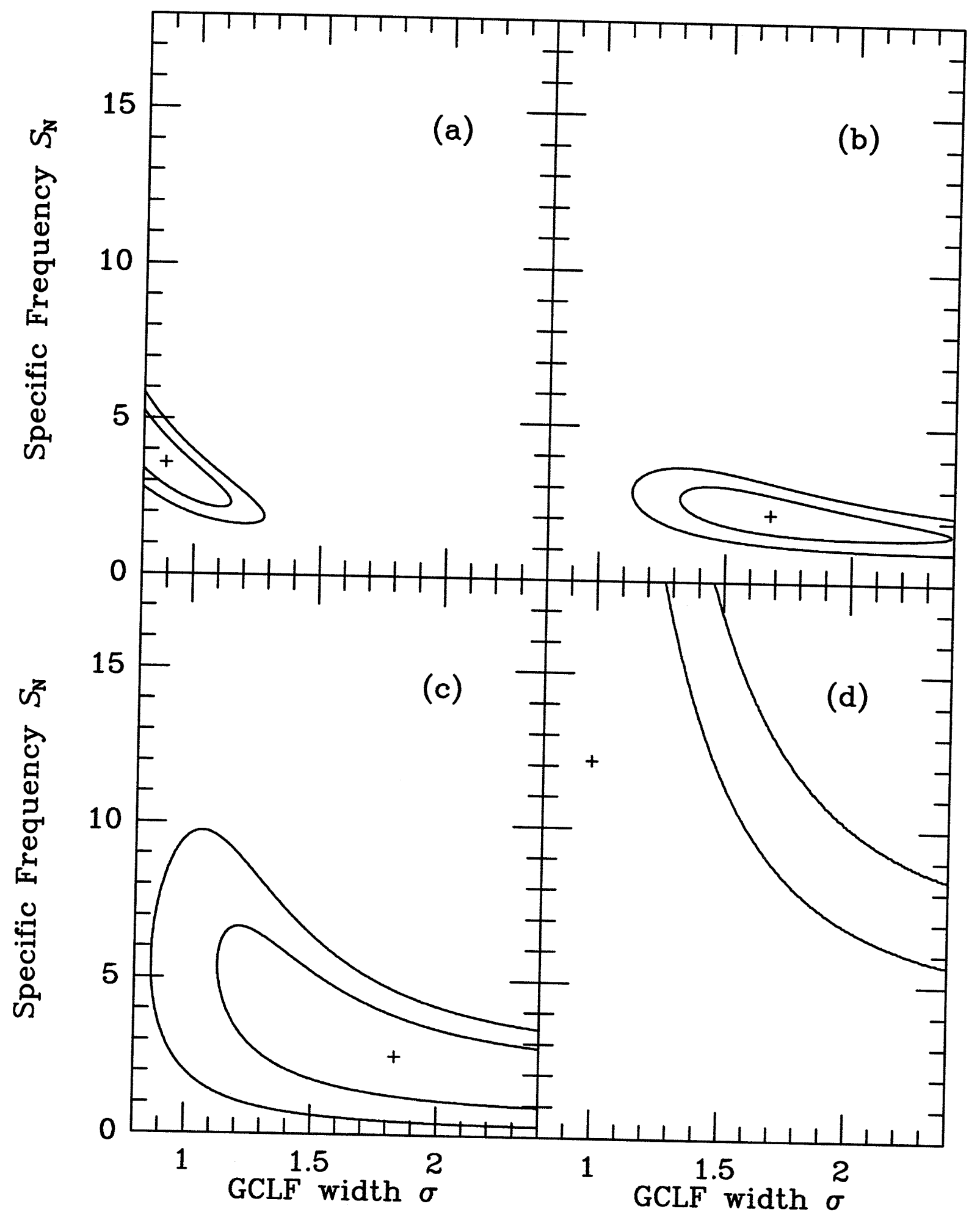

Figure A-7: A569-1 (0295). 


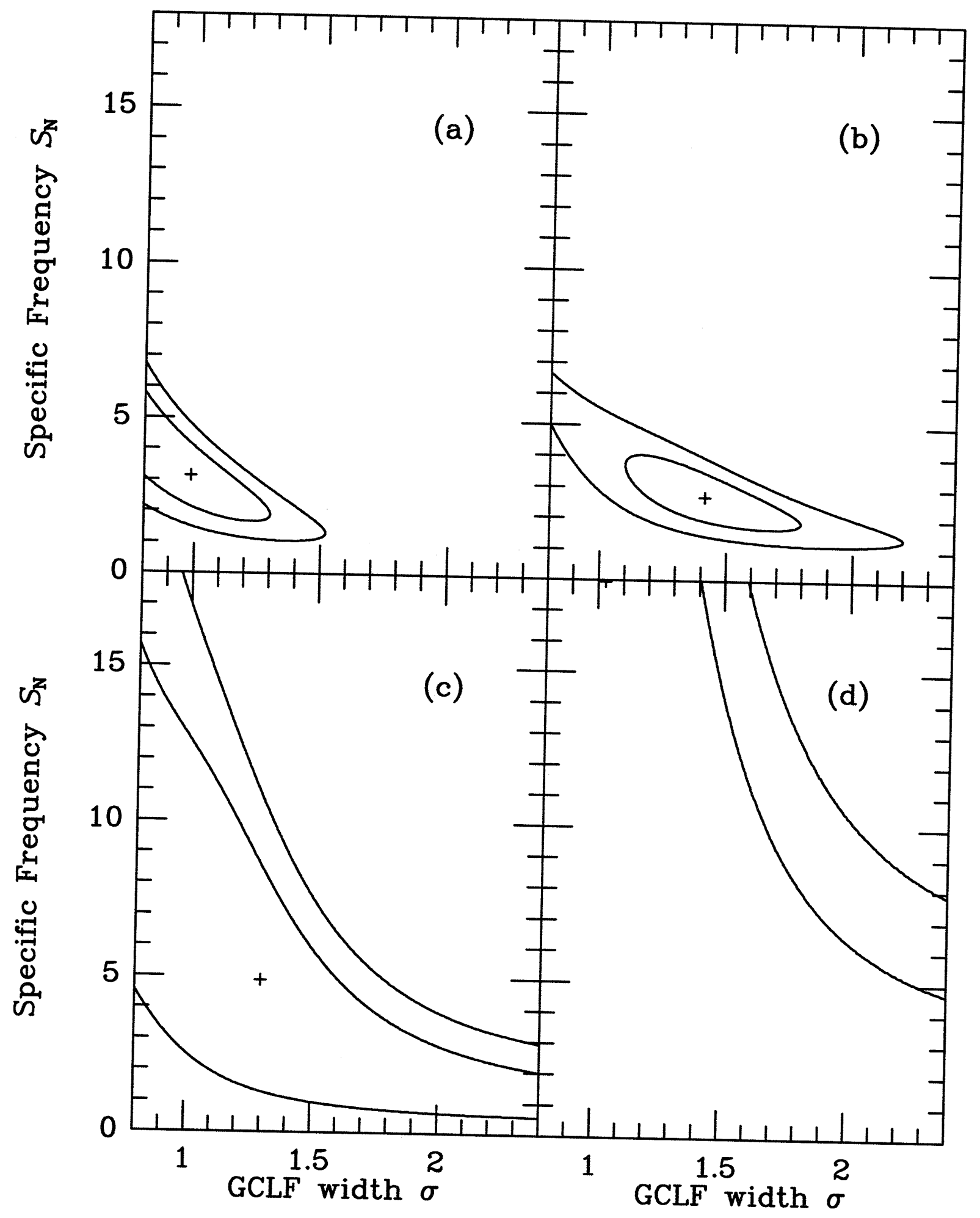

Figure A-8: A569-1 (0395). 


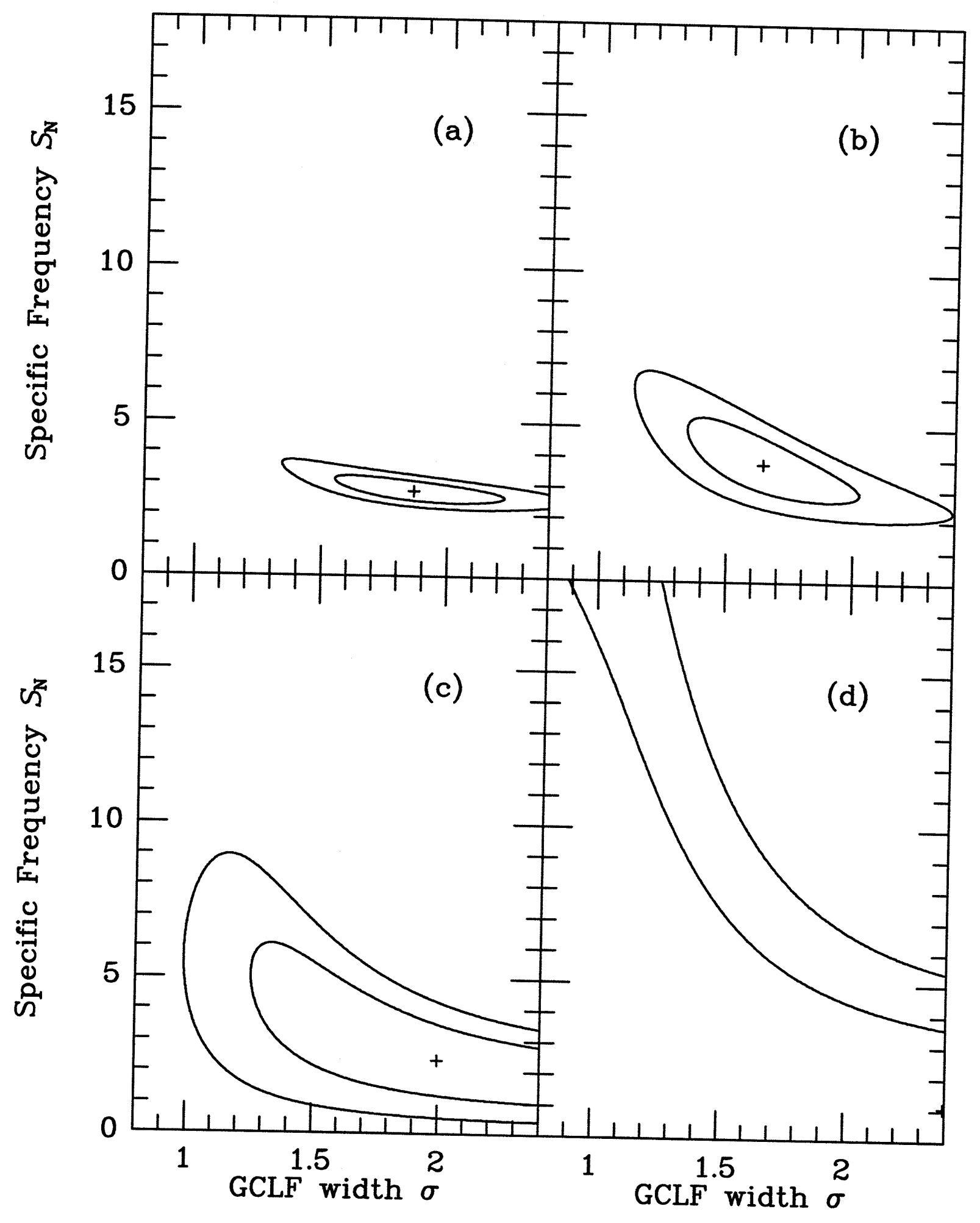

Figure A-9: A634-1. 


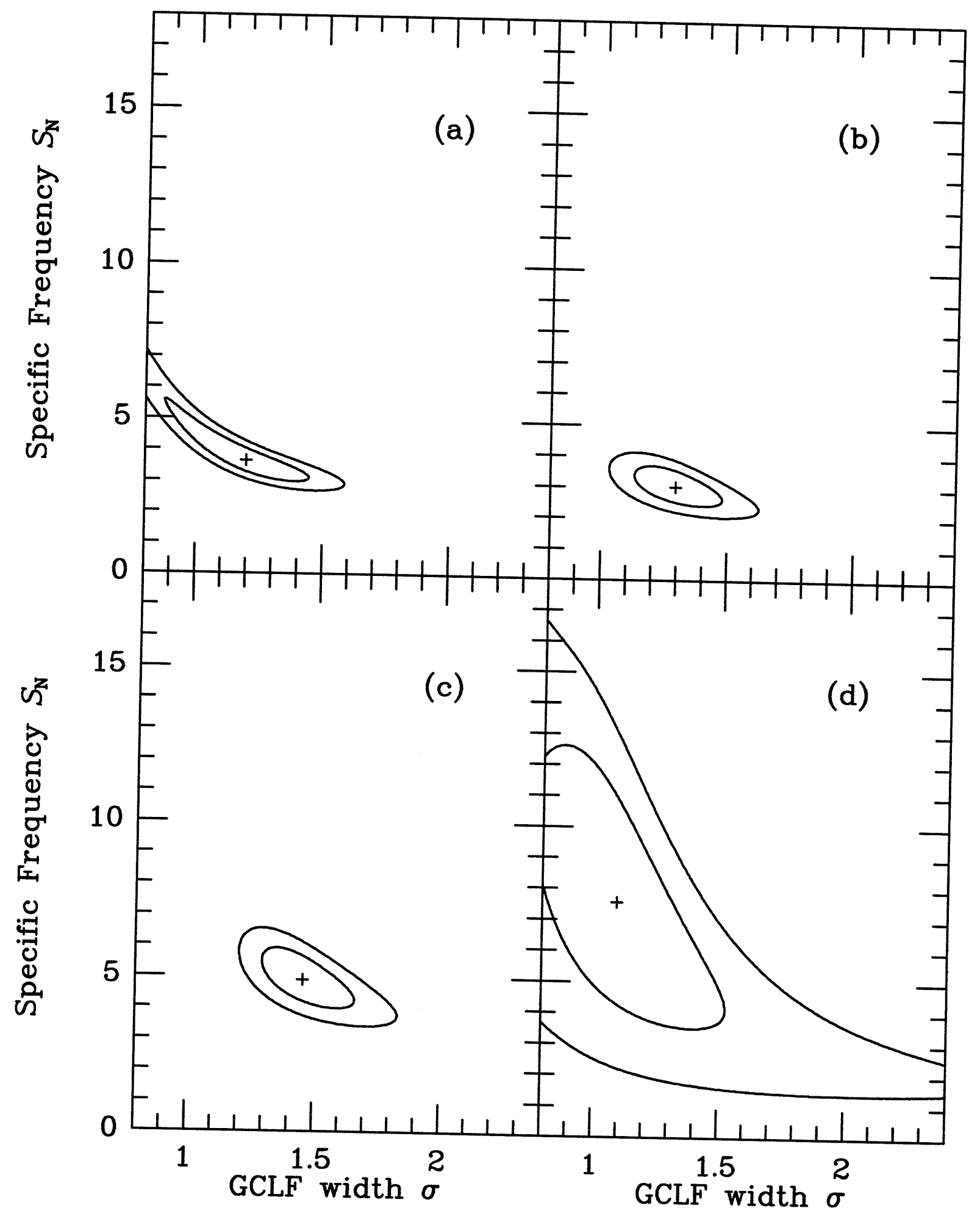

Figure A-10: A779-1. 


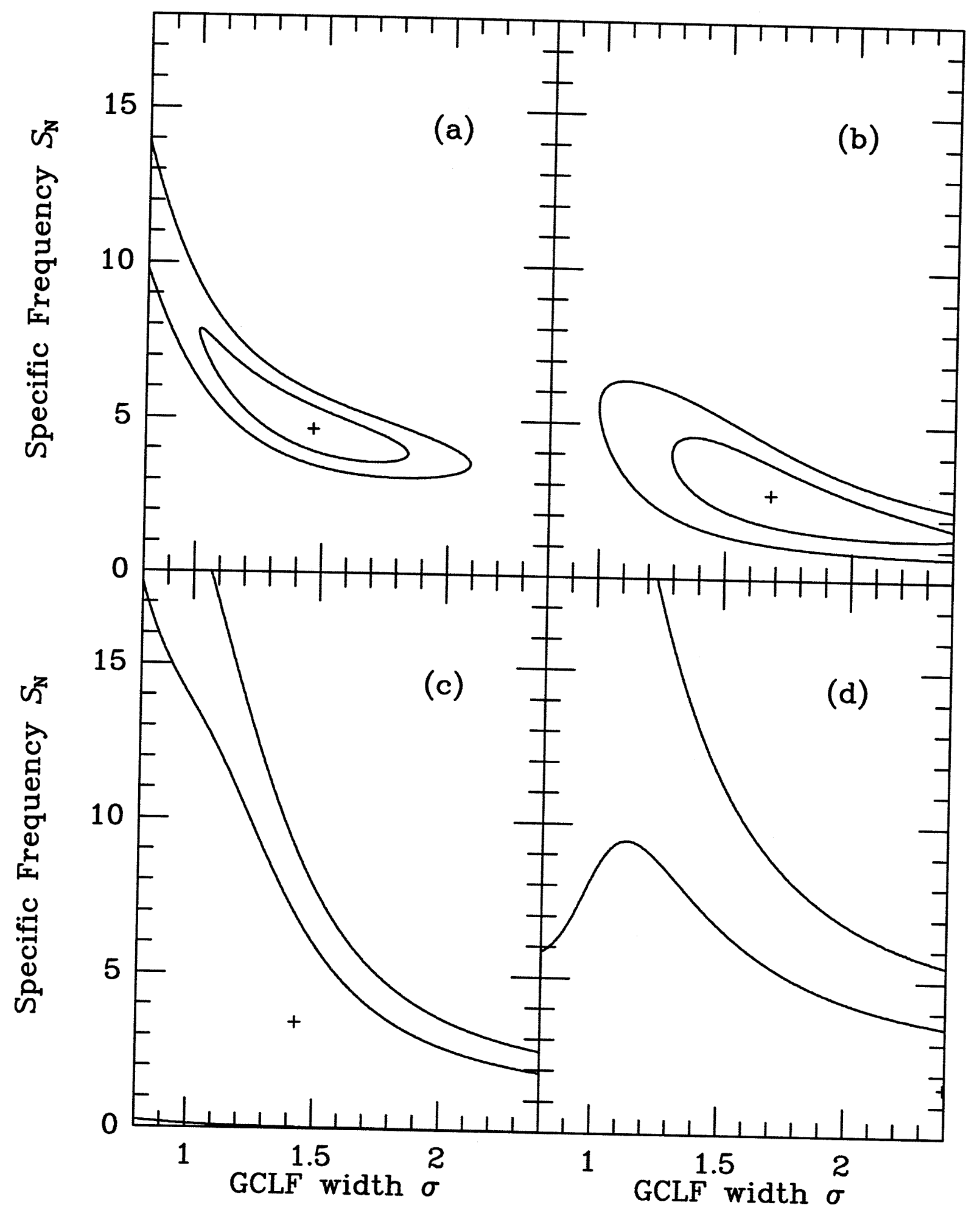

Figure A-11: A999-1. 


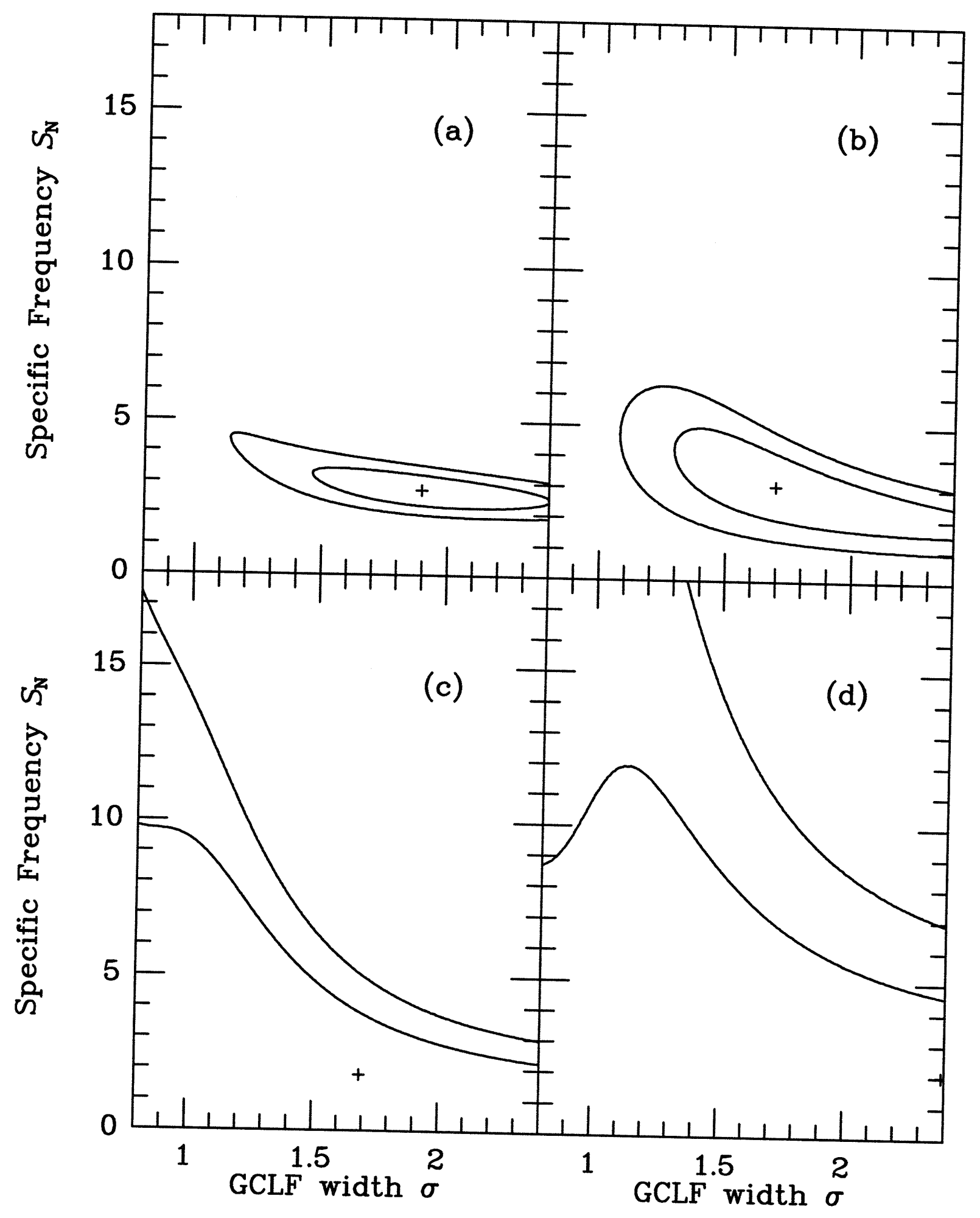

Figure A-12: A1016-1. 


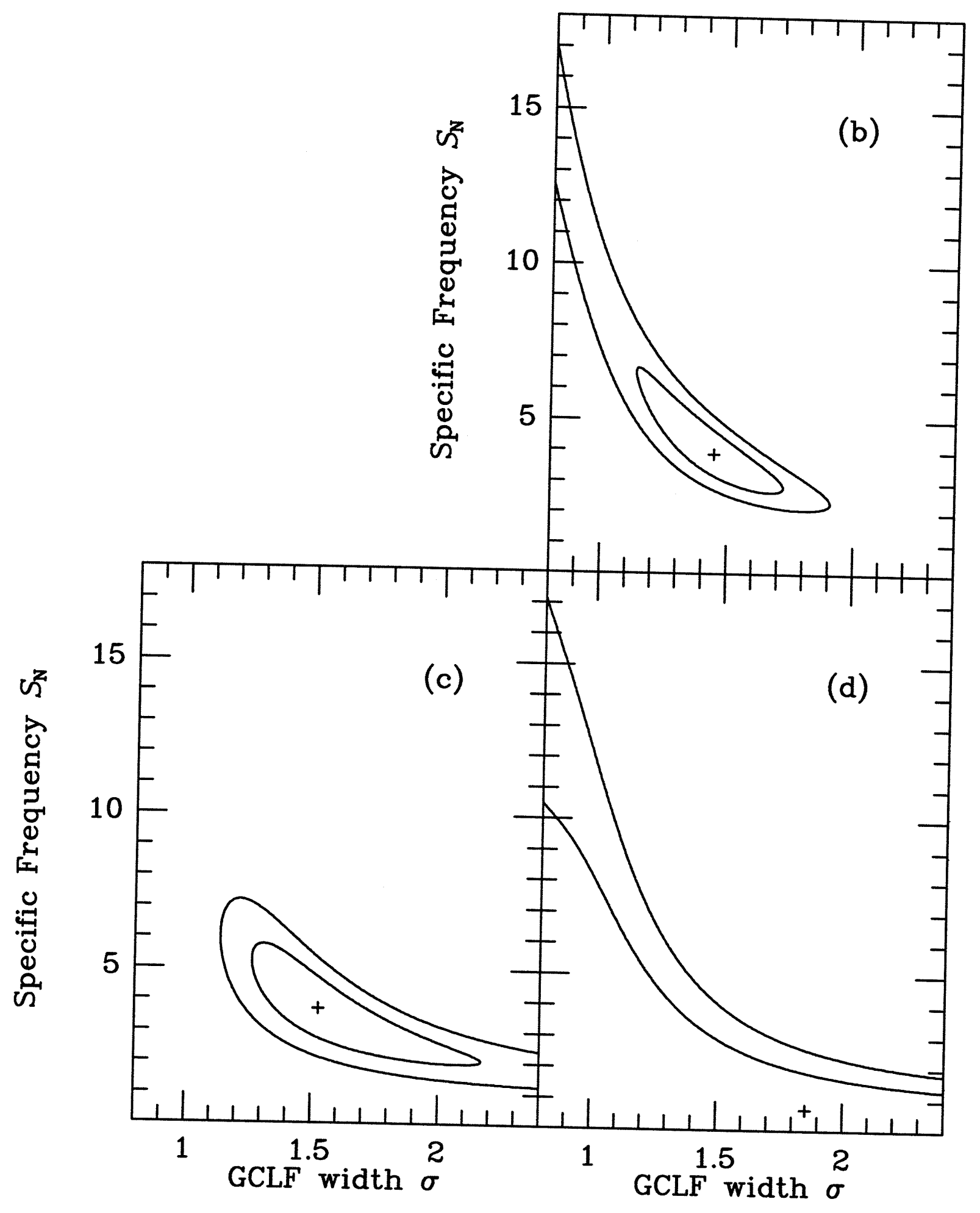

Figure A-13: A1177-1. 


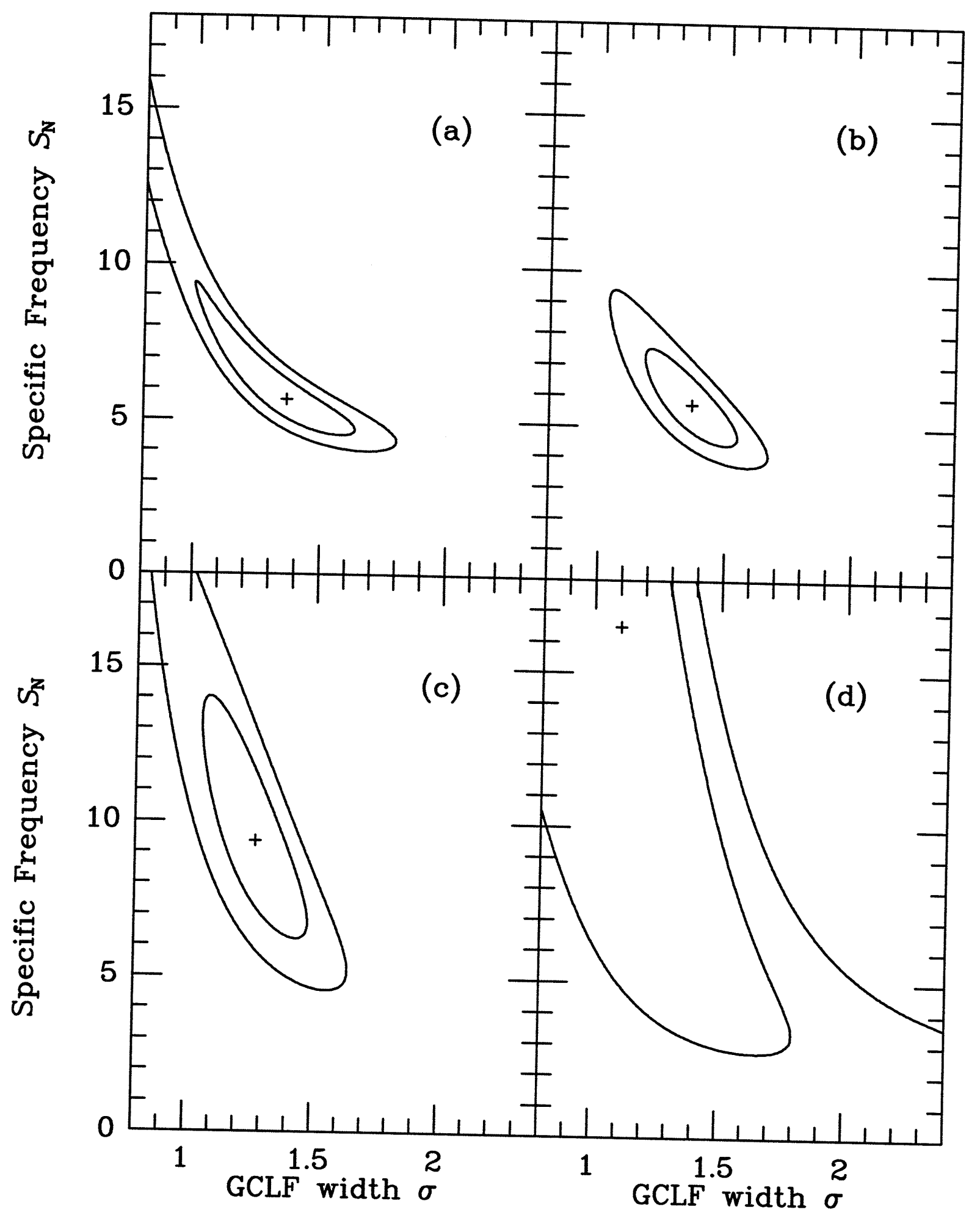

Figure A-14: A1185-1. 


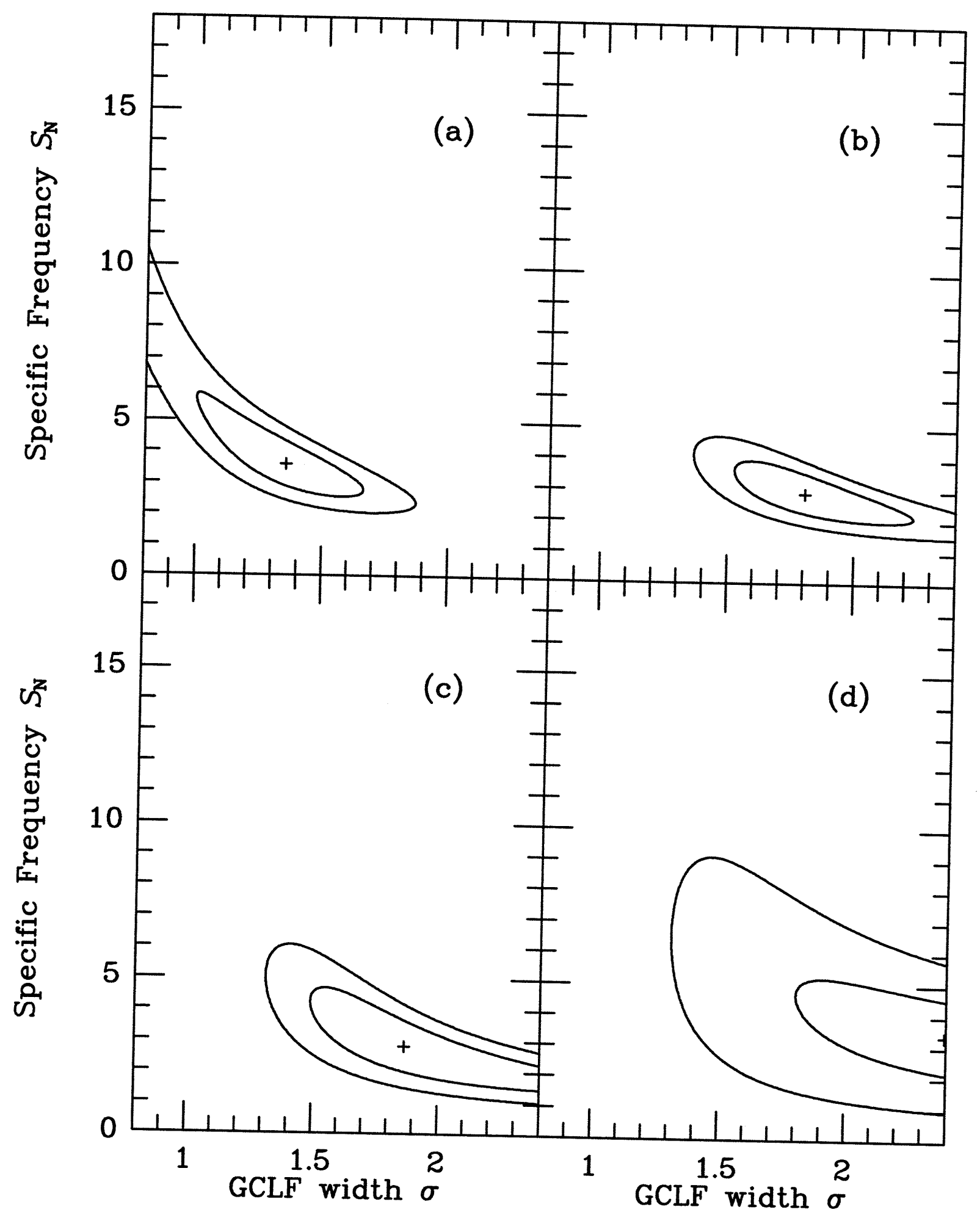

Figure A-15: A1314-1. 


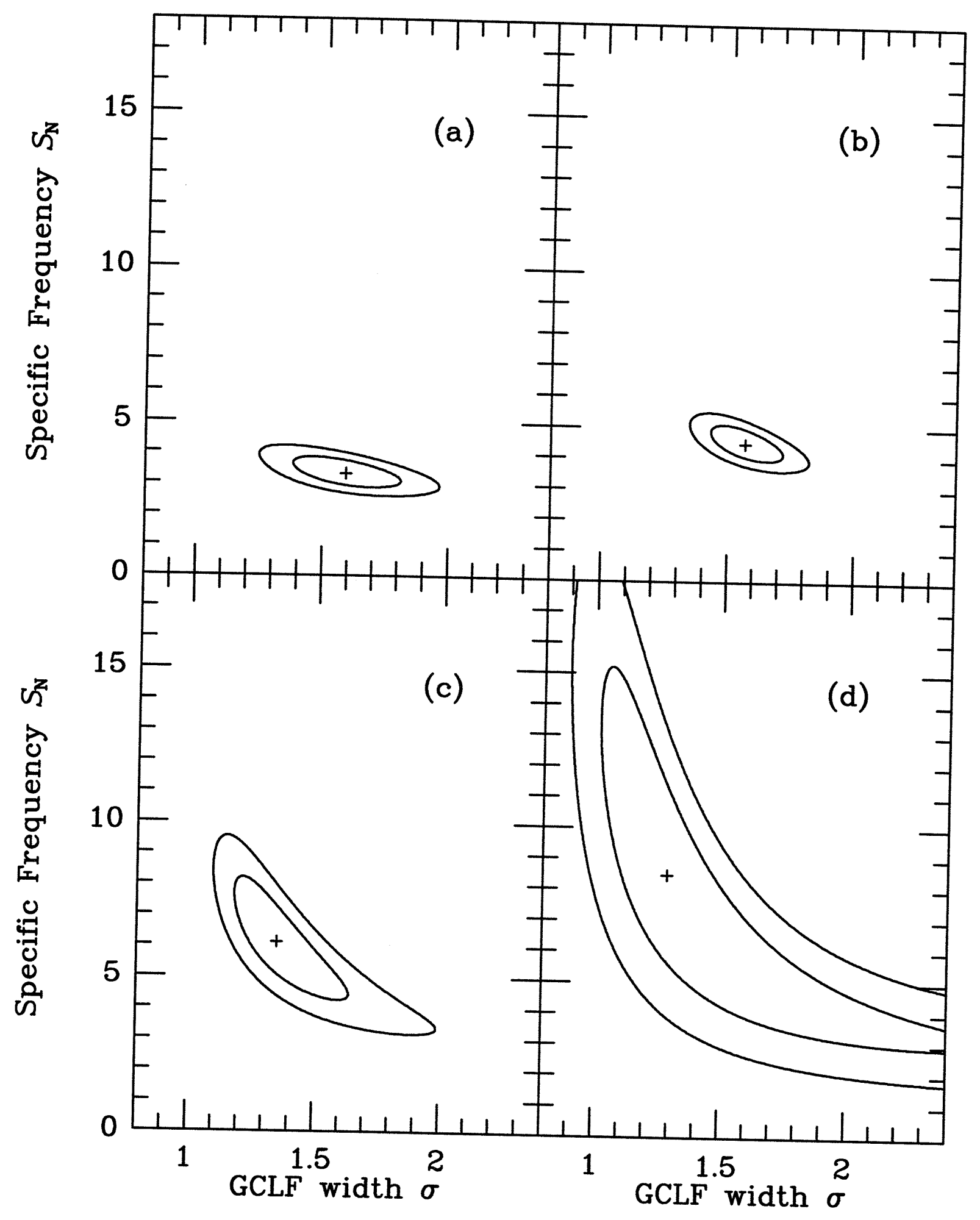

Figure A-16: A1367-1. 


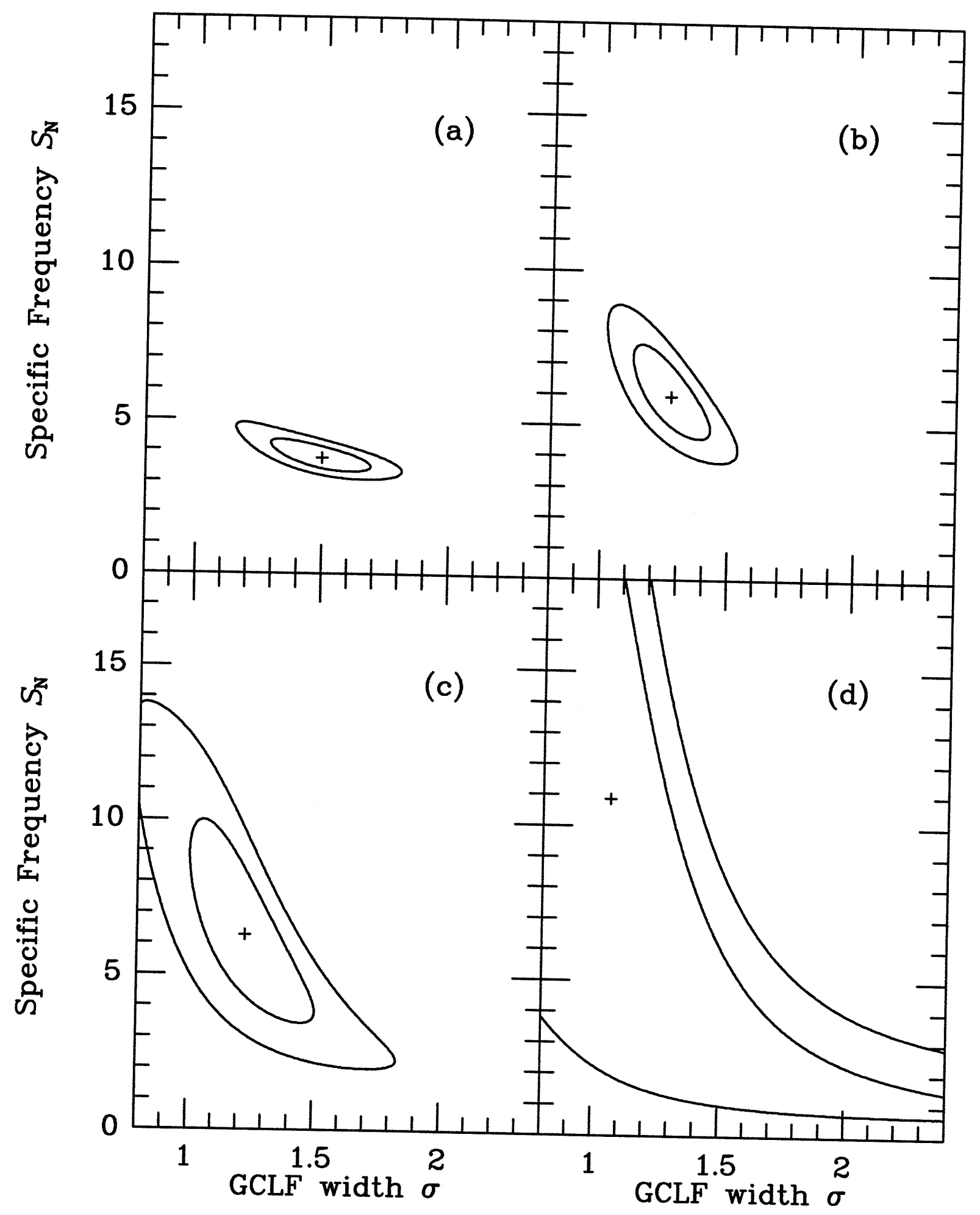

Figure A-17: A1656-3. 


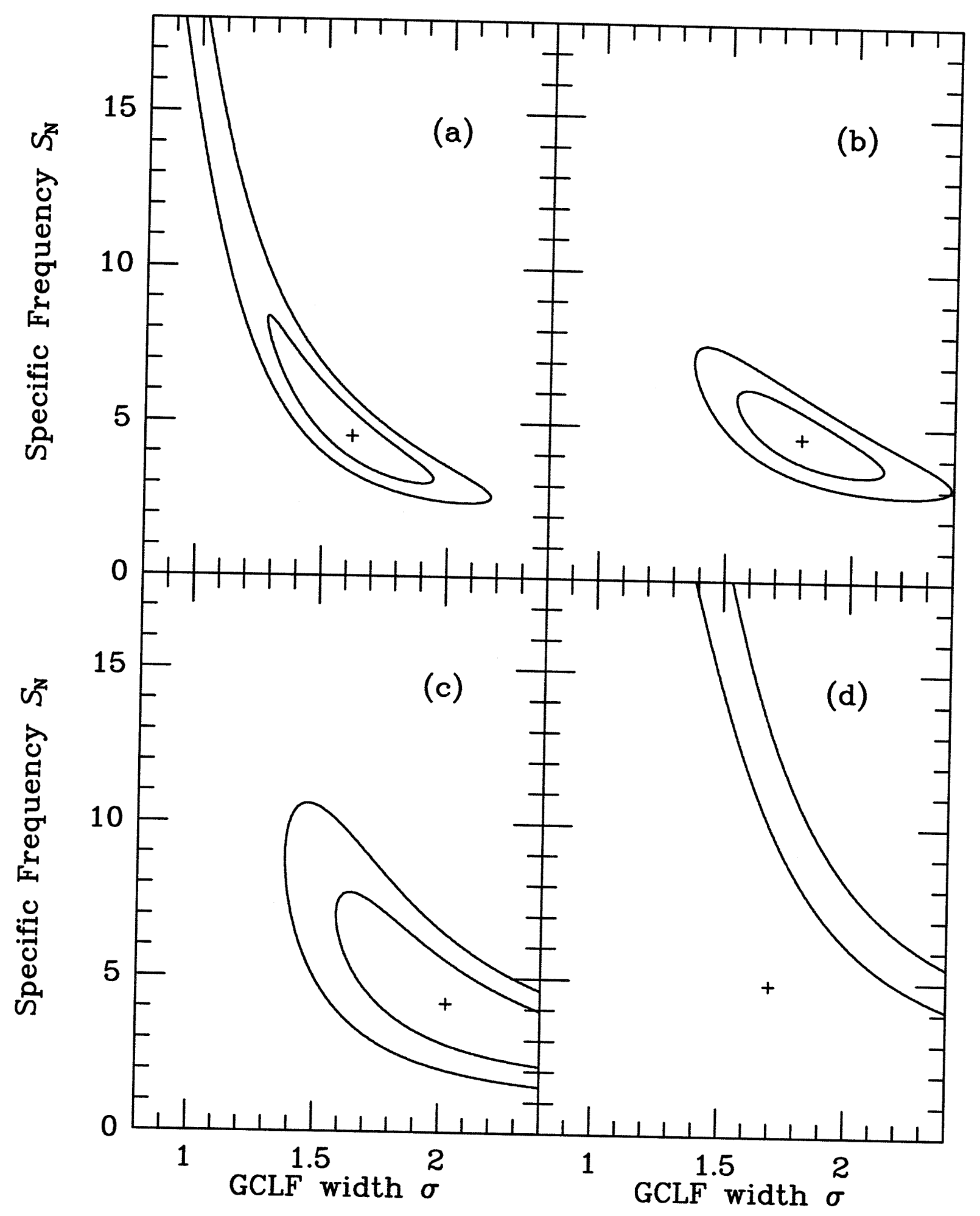

Figure A-18: A2162-1. 


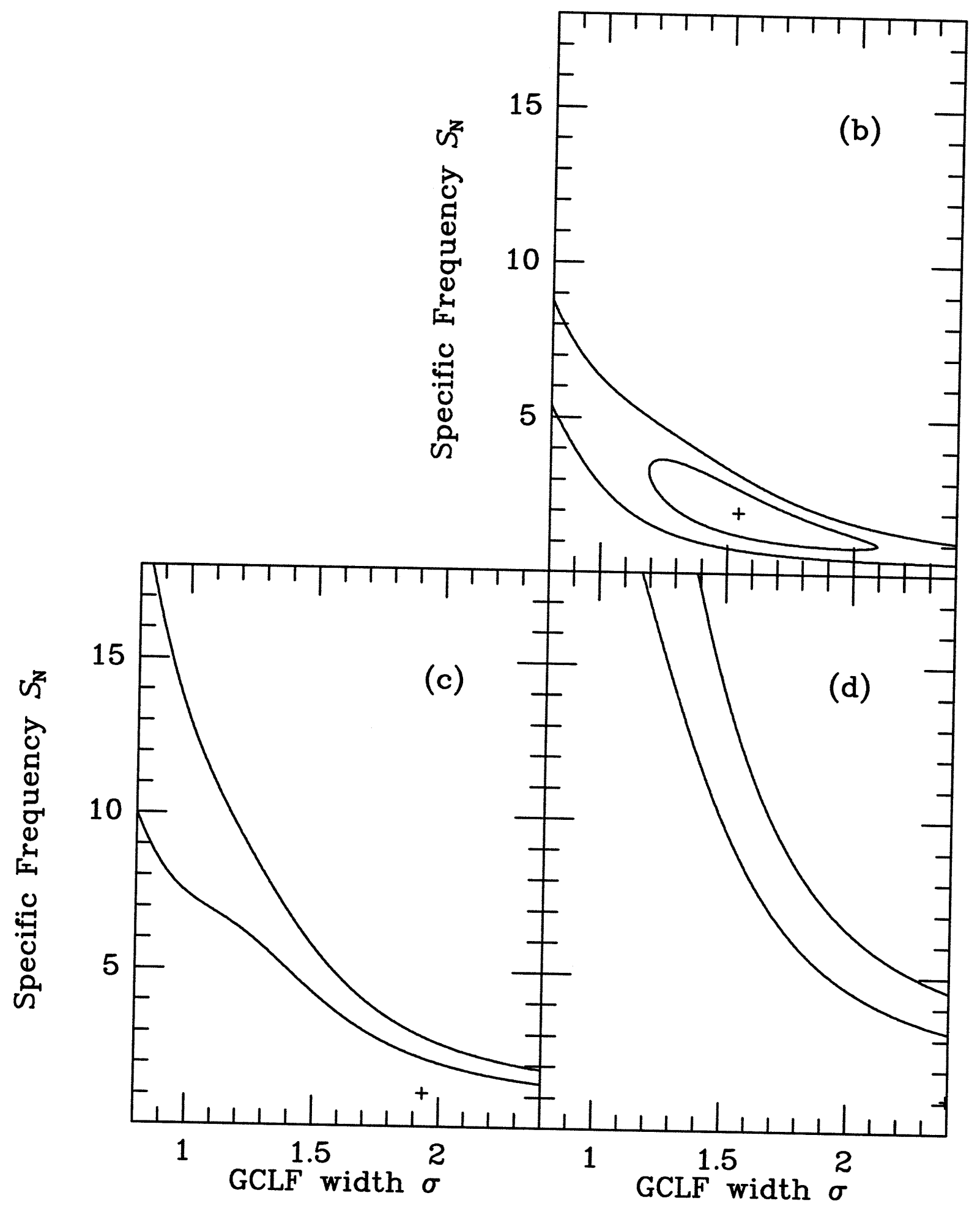

Figure A-19: A2197-1. 


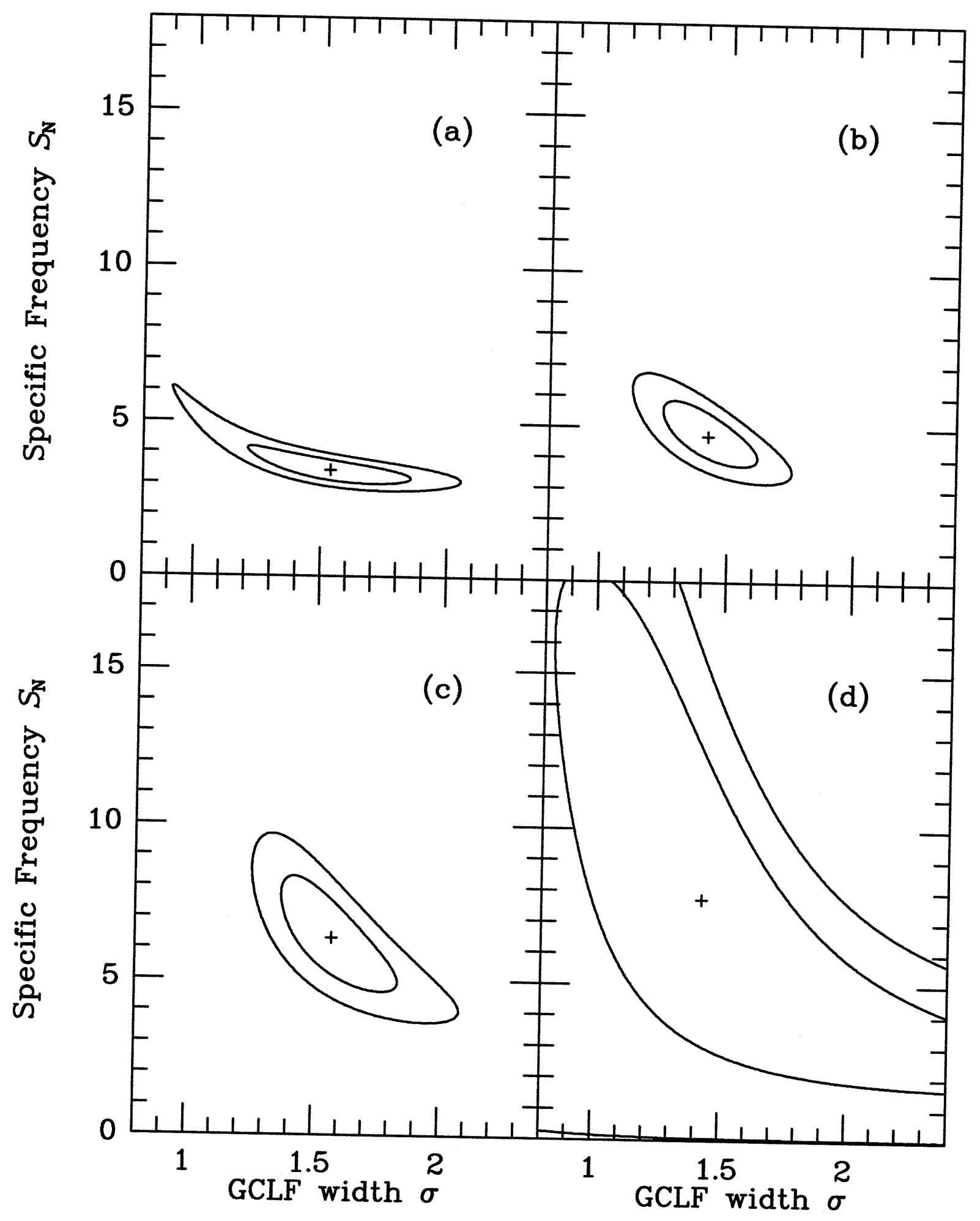

Figure A-20: A2197-2. 


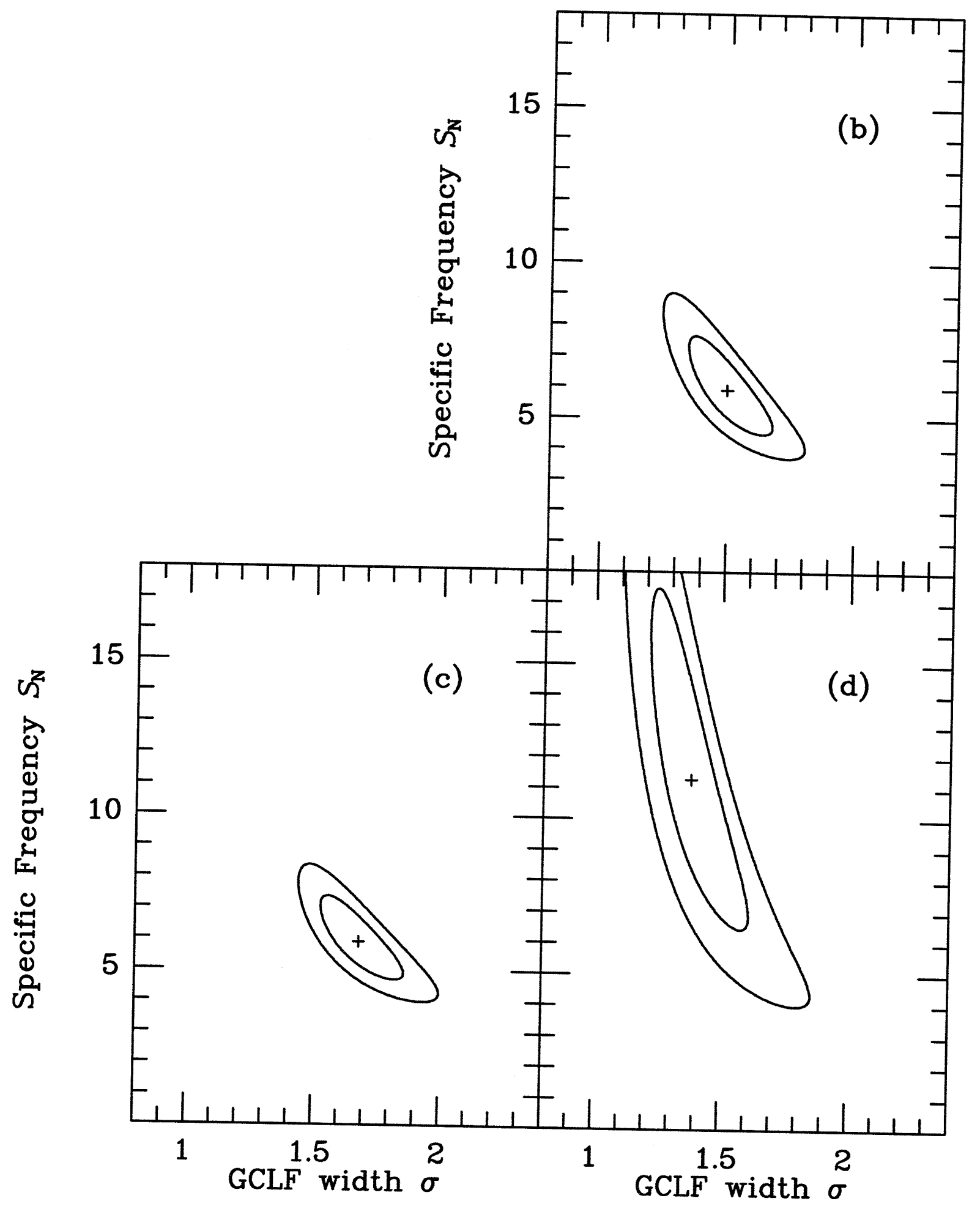

Figure A-21: A2199-1. 


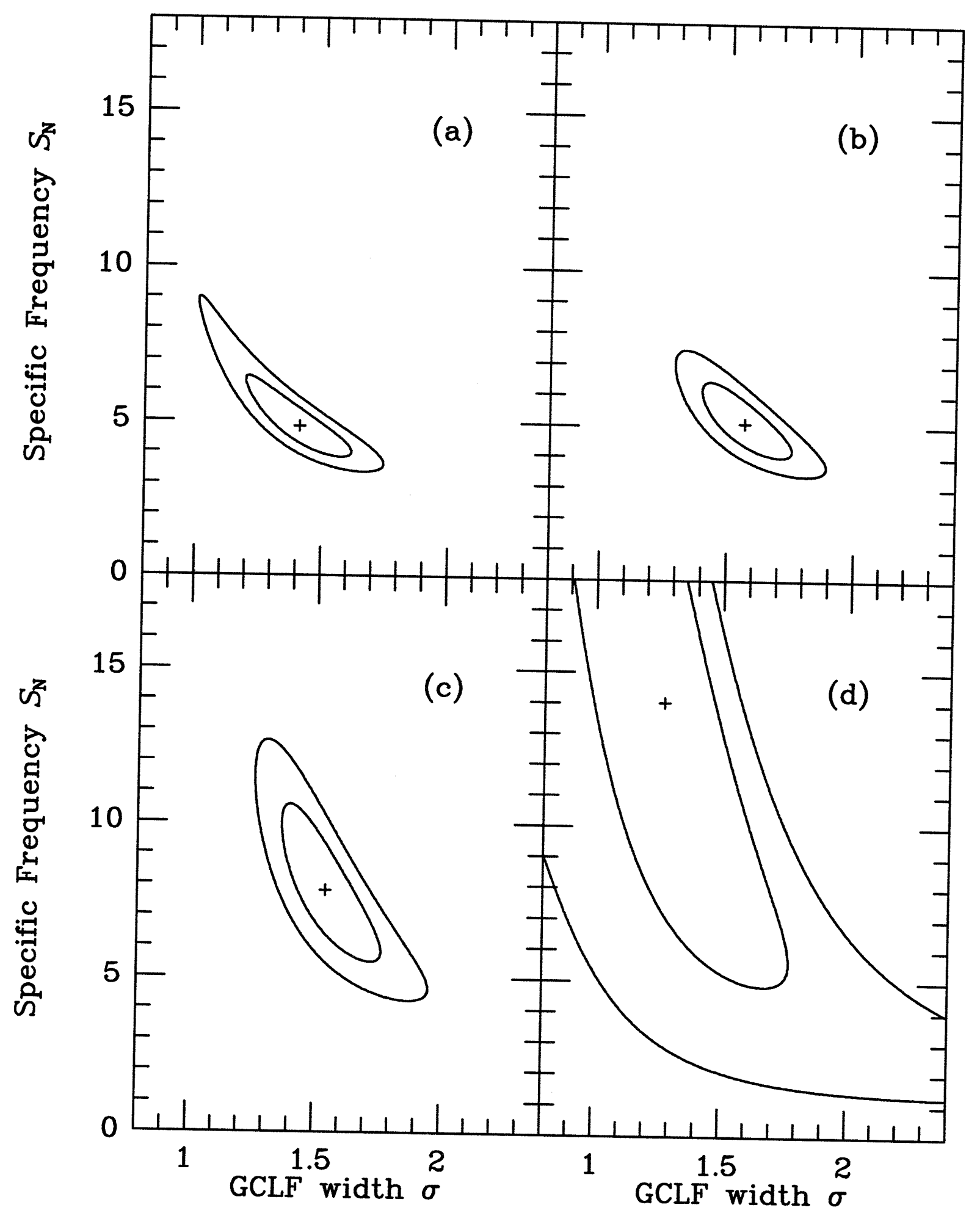

Figure A-22: A2634-1. 


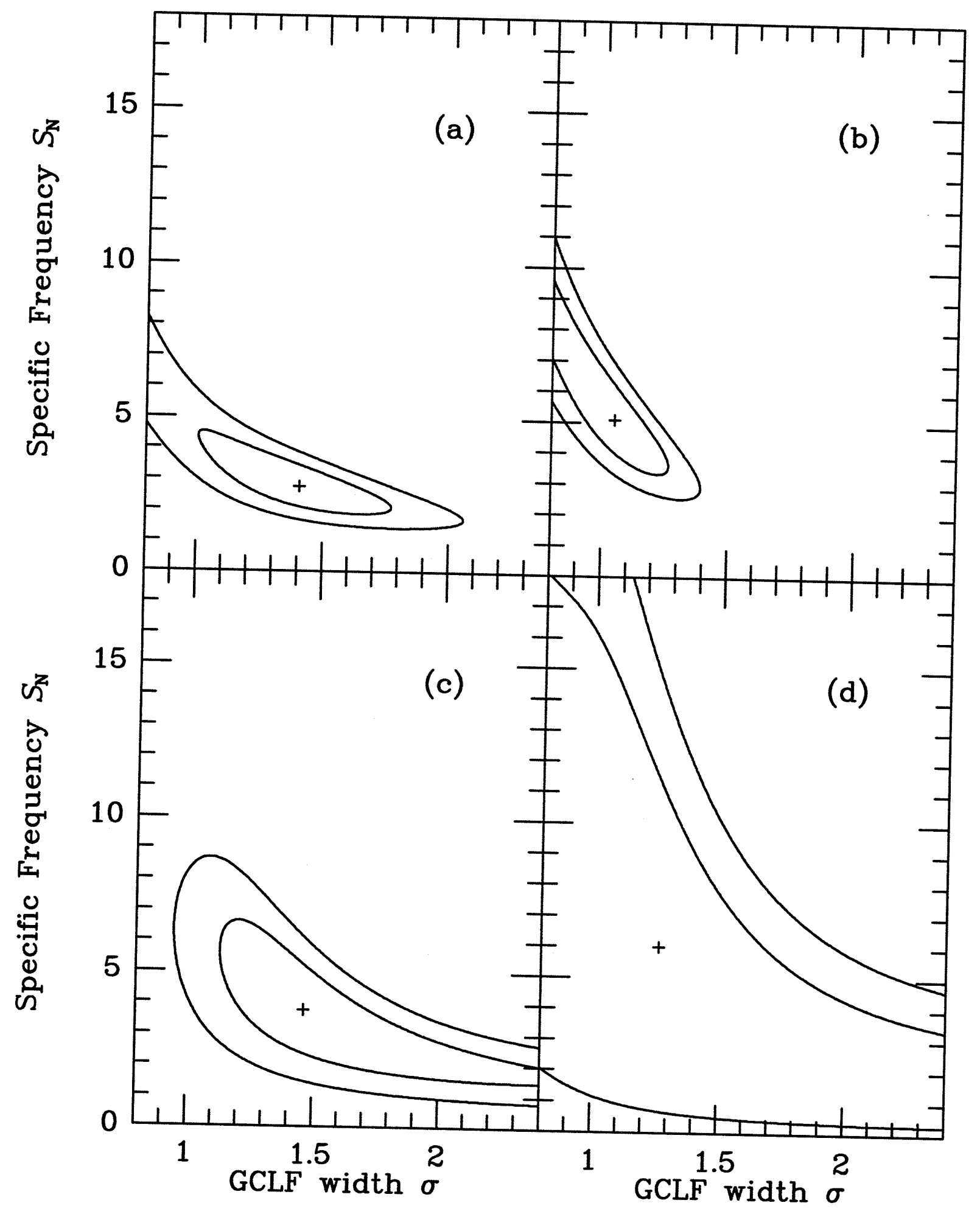

Figure A-23: A2666-1. 